\title{
Classification in the Presence of Missing Covariates
}

by

\section{Zahra Montazeri-Najafabadi}

\author{
A thesis submitted to \\ the Faculty of Graduate Studies and Research \\ in partial fulfillment of the requirements for the degree of
}

Doctor of Philosophy

School of Mathematics and Statistics

Ottawa-Carleton Institute for Mathematics and Statistics

Carleton University

Ottawa, Ontario, Canada

April, 2007

(C) Copyright 2007

Zahra Montazeri-Najafabadi 


$\begin{array}{ll}\begin{array}{l}\text { Library and } \\ \text { Archives Canada }\end{array} & \begin{array}{l}\text { Bibliothèque et } \\ \text { Archives Canada }\end{array} \\ \begin{array}{l}\text { Published Heritage } \\ \text { Branch }\end{array} & \begin{array}{l}\text { Direction du } \\ \text { Patrimoine de l'édition }\end{array} \\ \begin{array}{l}\text { 395 Wellington Street } \\ \text { Ottawa ON K1A ON4 }\end{array} & \begin{array}{l}\text { 395, rue Wellington } \\ \text { Ottawa ON K1A ON4 } \\ \text { Canada }\end{array}\end{array}$

Your file Votre référence ISBN: 978-0-494-33503-1 Our file Notre référence ISBN: 978-0-494-33503-1

NOTICE:

The author has granted a nonexclusive license allowing Library and Archives Canada to reproduce, publish, archive, preserve, conserve, communicate to the public by telecommunication or on the Internet, loan, distribute and sell theses worldwide, for commercial or noncommercial purposes, in microform, paper, electronic and/or any other formats.

The author retains copyright ownership and moral rights in this thesis. Neither the thesis nor substantial extracts from it may be printed or otherwise reproduced without the author's permission.
AVIS:

L'auteur a accordé une licence non exclusive permettant à la Bibliothèque et Archives Canada de reproduire, publier, archiver, sauvegarder, conserver, transmettre au public par télécommunication ou par l'Internet, prêter, distribuer et vendre des thèses partout dans le monde, à des fins commerciales ou autres, sur support microforme, papier, électronique et/ou autres formats.

L'auteur conserve la propriété du droit d'auteur et des droits moraux qui protège cette thèse. $\mathrm{Ni}$ la thèse ni des extraits substantiels de celle-ci ne doivent être imprimés ou autrement reproduits sans son autorisation.
In compliance with the Canadian

Privacy Act some supporting forms may have been removed from this thesis.

While these forms may be included in the document page count, their removal does not represent any loss of content from the thesis.
Conformément à la loi canadienne sur la protection de la vie privée, quelques formulaires secondaires ont été enlevés de cette thèse.

Bien que ces formulaires aient inclus dans la pagination, il n'y aura aucun contenu manquant.

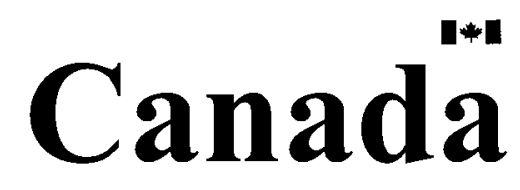


To my parents

for their love 


\section{Abstract}

The purpose of this thesis is to study the problem of classification in the presence of missing covariates and to propose methods for constructing consistent classifiers under various missing patterns. We derive representations for the best classifier when some of the covariates can be missing; this is done without imposing any assumptions on the underlying missing probability mechanism. Furthermore, without assuming any MAR-type conditions, we also construct consistent classifiers that do not require any imputation-based techniques. When the MAR assumption holds, we employ kernel-based imputation and Horvitz-Thompson-type inverse weighting approaches to handle the presence of missing covariates. The validity of our resulting classifiers is assessed via both theory and numerical examples. The thesis is organized as follows:

Chapter 1 contains the basic definitions and concepts which will be needed throughout this thesis . Here, we introduce some specific notation while discussing a few preliminary results and a brief description about the literature of the problem which is to be discussed in this thesis. Chapter 2 gives two representations of the functional form of the optimal classifier when a block of covariates is missing; it also proposes consistent parametric and nonparametric classifiers. In Chapter 3 we introduce the Swiss-cheese model where missing values can be anywhere among the covariates. In this chapter we derive the best classifier and construct consistent classifiers under various conditions. Chapter 4 applies the results from Chapter 2 and 3 to find consistent classifiers based on histogram rules as well as the general partitioning 
rules. In Chapter 5 the least-squares approach is used to perform nonparametric classification in the presence of missing covariates. In this chapter both kernel-based imputation as well as Horvitz-Thompson-type inverse weighting approaches are employed to handle the presence of missing covariates. Using the theory of empirical processes, the performance of the resulting classifiers is assessed by obtaining exponential bounds on the deviations of their conditional misclassification errors from that of the best classifier. Chapter 6 contains some simulation studies to illustrate the proposed methods. In this chapter, we consider artificial examples as well as real data sets. Finally, Chapter 7 suggests some possible future studies. 


\section{Acknowledgements}

First and foremost, my sincere gratitude and thanks go to my thesis supervisor, Professor Mojirsheibani, whose years of invaluable support are what got me here today. He spent countless hours revising my work, giving me very useful feedback and without his help, I would not have been able to complete my thesis. He persuaded me to continue on when I did not think I could and I would like to express my appreciation for his great patience and kindness together with endless enthusiasm for my research. Professor Mojirsheibani has selflessly guided me on this adventure and has helped me achieve my goals and dreams.

I would also like to thank Professors M. Asgharian, M.Alvo, J.N.K Rao, and D.R Thomas for serving as members of my advisory committee.

A very special thanks goes to Ann Woodside, for the time and effort she put into reading the manuscript and providing me with very helpful comments.

I am also very grateful to the School of Mathematics and Statistics at Carleton University for providing me with a very friendly working environment. Many thanks go to the staff and Professors of the Mathematics and Statistics department for the help they have given me and for making my time at Carleton a memorable experience.

Of course, I would like to thank my kids, Negar, Nima, and Arash, for their understanding, patience and unconditional love. Each one of them has a very special place in my heart for the little things that gave me the confidence to continue on the path to success. 
I wish to dedicate this thesis to my loving parents. Their countless sacrifices and encouraging acts throughout the years are what formed the foundation of my work. 


\section{Contents}

Abstract $\quad$ i

Acknowledgements

Contents $\quad$ v

List of Tables $\quad$ ix

List of Figures $\quad$ x

1 Background And Literature Review 1

1.1 Introduction . . . . . . . . . . . . . . . . . . . 1

1.2 Statistical Classification Setup . . . . . . . . . . . . . 2

1.3 Classification Rules ................... 5

1.3.1 Parametric Rules . . . . . . . . . . . . . . . 6

1.3.2 Linear Discriminant Rules ... . . . . . . . . . 7

1.3.3 Nonparametric Rules . . . . . . . . . . . . . . . . 8

1.4 Selecting Classifiers . . . . . . . . . . . . . . . . 14

1.5 Missing Covariates . . . . . . . . . . . . . . 22

1.5.1 Methods for Analyzing Missing Data . . . . . . . . . . . 24

$\mathrm{V}$ 


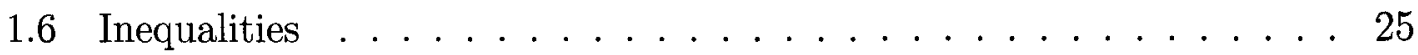

1.7 Classification with Missing Data . . . . . . . . . . . . . 26

1.8 Thesis Outline . . . . . . . . . . . . . . . . . 28

2 The Best Classifier in the Presence of Missing Covariates 30

2.1 Introduction . . . . . . . . . . . . . . . . 30

2.2 The Best Classifier . . . . . . . . . . . . . . . . . 31

2.3 Sample Versions of the Best Classifier . . . . . . . . . . . . . 43

2.4 Parametric Estimation . . . . . . . . . . . . . . . . 45

2.4 .1 Parameters Estimation . . . . . . . . . . . . . . . 47

2.4.2 Missing Probabilities Estimation _. . . . . . . . . 48

2.5 Nonparametric Estimation . . . . . . . . . . . . . . . . . 50

3 The Best Classifier for the Swiss-Cheese Model 55

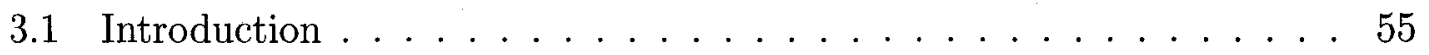

3.2 General Setup for the Swiss-Cheese Model . . . . . . . . . . . 56

3.3 The Best Classifier for the Swiss-Cheese Model . . . . . . . . . . . 59

3.4 Data-based Classifiers for the Swiss-Cheese Model . . . . . . . . . . 63

3.4.1 Parametric Estimation . . . . . . . . . . . . . . . 64

3.4 .2 Nonparametric Estimation . . . . . . . . . . . . . . 68

4 Partitioning Rules in the Presence of Missing Covariates $\quad 73$

4.1 Introduction . . . . . . . . . . . . . . . . 73

4.2 The Regular Histogram Rule When a Block of Covariates is Missing - 74

4.3 The Regular Histogram Rule for the Swiss-Cheese Model . . . . . . . 79

4.4 The General Partitioning Rule When a Block of Covariates is Missing 82

4.5 The General Partitioning Rule for the Swiss-Cheese Model . . . . 88

vi 
5 The Least-Squares Approach $\quad 92$

5.1 Introduction . . . . . . . . . . . . . . . 92

5.2 Minimizing the Empirical Squared Error in the MAR Case . . . . . . 93

5.2.1 A Hypothetical Situation . . . . . . . . . . . . . 95

5.2 .2 Kernel-Based Imputation . . . . . . . . . . . . . . . 102

5.3 Horvitz-Thompson-Type Estimators . . . . . . . . . . . . . . . . . . 114

5.4 Minimizing the Empirical Squared Error in the NMAR Case .. . . . 126

6 Simulation Studies $\quad 133$

6.1 Introduction . . . . . . . . . . . . . . . 133

6.2 Effects of Missing Probability Mechanism . . . . . . . . . . . . 134

6.3 Parametric and Nonparametric Classification For Simulated Data . . 144

6.4 A Real Data Set . . . . . . . . . . . . . . . . . 161

$\begin{array}{lll}7 & \text { Future Research } & 170\end{array}$

$\begin{array}{lr}\text { Bibliography } & 172\end{array}$ 


\section{List of Tables}

2.1 Error rates for $\psi_{B}$ and $\psi_{\text {naïve }}$ when $p=0.5 \ldots \ldots . \ldots 40$

2.2 Error rates for $\psi_{B}$ and $\psi_{\text {naïve }}$ when $p=0.85 \ldots \ldots . \ldots 41$

2.3 Error rates for $\psi_{B}$ and $\psi_{\text {naïve }}$ when $p=0.25 \ldots \ldots$. . . . . . . 42

6.1 Error rates for $\psi_{B}$ and $\psi_{\text {naïve }}$ when $\sigma_{x, v}=0.15$ and $p=0.5 \ldots 136$

6.2 Error rates for $\psi_{B}$ and $\psi_{\text {naive }}$ when $\sigma_{x v}=0.8$ and $p=0.5 \ldots 137$

6.3 Error rates for $\psi_{B}$ and $\psi_{\text {naïve }}$ when $\sigma_{x v}=0$ and $p=0.5 \ldots 138$

6.4 Error rates for $\psi_{B}$ and $\psi_{\text {naïve }}$ when $\sigma_{x v}=0.15$ and $p=0.25 \ldots 139$

6.5 Error rates for $\psi_{B}$ and $\psi_{\text {naïve }}$ when $\sigma_{x v}=0.8$ and $p=0.25 \ldots 140$

6.6 Error rates for $\psi_{B}$ and $\psi_{\text {naïve }}$ when $\sigma_{x v}=0$ and $p=0.25 \ldots 141$

6.7 Error rates for $\psi_{B}$ and $\psi_{\text {naive }}$ when $\sigma_{x v}=0.15$ and $p=0.85 \ldots 142$

6.8 Error rates for $\psi_{B}$ and $\psi_{\text {naïve }}$ when $\sigma_{x v}=0.8$ and $p=0.85 \ldots 143$

6.9 Error rates for $\psi_{B}$ and $\psi_{\text {naïv }}$ when $\sigma_{x v}=0$ and $p=0.85 \ldots \ldots 144$

6.10 Error rates for $\widehat{\psi}_{n}$ (MLE) and $\psi_{n}^{\text {ker }}$ (nonparametric) when $\sigma_{x v}=0.15 .148$

6.11 Error rates for $\widehat{\psi}_{n}$ (MLE) and $\psi_{n}^{\mathrm{ker}}$ (nonparametric) when $\sigma_{x v}=0.8 . \quad 150$

6.12 Error rates for $\widehat{\psi}_{n}$ (MLE) and $\psi_{n}^{\text {ker }}$ (nonparametric) when $\sigma_{x v}=0 . \quad .152$

6.13 Error rates for $\widehat{\psi}_{n}$ (MLE) and $\psi_{n}^{\text {hist }}$ (nonparametric) when $\sigma_{x v}=0.15 .156$

6.14 Error rates for $\widehat{\psi}_{n}$ (MLE) and $\psi_{n}^{\text {hist }}$ (nonparametric) when $\sigma_{x v}=0.8 .158$

6.15 Error rates for $\widehat{\psi}_{n}$ (MLE) and $\psi_{n}^{\text {hist }}$ (nonparametric) when $\sigma_{x v}=0 . .160$

viii 
6.16 A portion of the data set . . . . . . . . . . 163

6.17 Description of variables . . . . . . . . . . . . . . 164

6.18 Error rates for $\psi_{n}^{\mathrm{ker}}, \widetilde{\psi}_{n}^{\mathrm{ker}}$, and $\bar{\psi}_{n}^{\mathrm{ker}}$, when $n=564 \ldots \ldots . \ldots 165$

6.19 Error rates for $\psi_{n}^{\mathrm{ker}}, \widetilde{\psi}_{n}^{\mathrm{ker}}$, and $\bar{\psi}_{n}^{\mathrm{ker}}$, when $n=400 \ldots \ldots . \ldots 165$

6.20 Error rates for $\psi_{n}^{\mathrm{ker}}, \widetilde{\psi}_{n}^{\mathrm{ker}}$, and $\bar{\psi}_{n}^{\mathrm{ker}}$, when $n=200 \ldots \ldots$ 


\section{List of Figures}

2.1 One block missing data pattern. Rows correspond to variables, columns to observations. $n=n_{1}+n_{2} \ldots \ldots 33$

3.1 Swiss-cheese pattern. Rows correspond to variables, columns to observations. $n=12 \ldots \ldots \ldots \ldots \ldots$

6.1 Plot of $h$ versus the error, o represents $\psi_{n}^{\text {ker }}, \bullet$ represents $\widetilde{\psi}_{n}^{\text {ker }} \ldots \ldots 167$

6.2 Plot of $h$ versus the error, o represents $\psi_{n}^{\text {ker }}$, • represents $\widetilde{\psi}_{n}^{\text {ker }}$. . . . 168

6.3 Plot of $h$ versus the error, o represents $\psi_{n}^{\text {ker }}, \bullet$ represents $\widetilde{\psi}_{n}^{\text {ker }}$. . . . 169 


\section{Chapter 1}

\section{Background And Literature}

\section{Review}

\subsection{Introduction}

This chapter contains two parts. In the first part we give a review of statistical classification problems where one is interested in predicting the unknown nature of an observation, based on available data. In the second part, we present a review of some classical approaches to missing values problems. Section 1.2 describes the statistical classification setup where classifiers, the best classifier, and misclassification error rates are defined. Section 1.3 reviews some common classification rules using both parametric and nonparametric methods. Section 1.4 describes the least-squares method for selecting a classifier from a certain class of classifiers and introduces Dudley-Pollard-type theorems. Section 1.5 briefly reviews statistical literature on the missing data problem, by explaining some fundamental definitions and methods. In Section 1.6, we review some important inequalities which are used 
repeatedly throughout this thesis. Section 1.7 briefly reviews previous studies related to classification with missing covariates, and Section 1.8 gives the outline of this thesis.

\subsection{Statistical Classification Setup}

In statistical classification, one is interested in guessing or predicting the unknown nature of an observation, usually a discrete quantity such as black or white, pass or fail, sick or healthy, rainy, sunny or cloudy, a group of diseases, etc. This observation is a collection of numerical measurements such as a vector of weather data. Formally, an observation is a $p$-dimensional vector $\mathbf{Z}$, also known as the feature vector or predictor vector, of observable measurements. The unknown class to which $\mathrm{Z}$ belongs is represented by the discrete random variable $Y$; in a $k$-class problem $Y$ takes values in $\{1, \cdots, k\}$. Throughout this thesis, we will focus on the popular two-class classification problem. More formally, we consider a random pair $(\mathbf{Z}, Y)$, where $\mathbf{Z} \in \mathcal{R}^{p}$ is observable and $Y \in\{0,1\}$ is to be predicted. Extensions to general multigroup problems are in most cases rather straightforward (although notationally more cumbersome).

Definition 1.2.1 (Classifier) A classifier $\psi$ is any map of the form $\psi: \mathcal{R}^{p} \rightarrow$ $\{0,1\}$, which is used to predict $Y$ given the feature vector $\mathbf{Z}$ [i.e., $\psi(\mathbf{Z}) \in\{0,1\}$ is the predicted value of $Y$ given $\mathbf{Z}$ ].

An error occurs if $\psi(\mathbf{Z}) \neq Y$ and the misclassification error rate of $\psi$, denoted by $L(\psi)$, is defined by

$$
L(\psi)=P\{\psi(\mathbf{Z}) \neq Y\}
$$


The best classifier, called the Bayes classifier and denoted by $\psi_{B}$, is the one that satisfies

$$
P\left\{\psi_{B}(\mathbf{Z}) \neq Y\right\}=\inf _{\psi: \mathcal{R}^{p} \rightarrow\{0,1\}} P\{\psi(\mathbf{Z}) \neq Y\} .
$$

If for any $\mathbf{z} \in \mathcal{R}^{p}$, we define

$$
\eta_{B}(\mathbf{z})=P\{Y=1 \mid \mathbf{Z}=\mathbf{z}\}=E(Y \mid \mathbf{Z}=\mathbf{z}),
$$

then the following theorem shows that the classifier $\psi_{B}$ defined by

$$
\psi_{B}(\mathbf{z})= \begin{cases}1 & \text { if } \eta_{B}(\mathbf{z})>1-\eta_{B}(\mathbf{z}) \\ 0 & \text { otherwise }\end{cases}
$$

is optimal.

Theorem 1.2.1 For any decision function $\psi: \mathcal{R}^{p} \rightarrow\{0,1\}$,

$$
P\left\{\psi_{B}(\mathbf{Z}) \neq Y\right\} \leqslant P\{\psi(\mathbf{Z}) \neq Y\}
$$

where $\psi_{B}$ defined in (1.1).

For a proof see, for example, Devroye et al., (1996) or Grörfi et al., (2002). In practice, one does not know the underlying distribution $F_{\mathbf{Z}, Y}$ of the pair $(\mathbf{Z}, Y)$, and therefore finding $\psi_{B}$ is virtually impossible. However, one typically has access to a random sample

$$
\mathcal{D}_{n}:=\left(\mathbf{Z}_{1}, Y_{1}\right),\left(\mathbf{Z}_{2}, Y_{2}\right), \cdots,\left(\mathbf{Z}_{n}, Y_{n}\right)
$$

where $\left(\mathbf{Z}_{i}, Y_{i}\right), i=1, \cdots, n$ are independent and identically distributed $\mathcal{R}^{p} \times\{0,1\}$ valued random vectors drawn from $F_{\mathbf{Z}, Y}$. This sample may have been observed in the past through experiments or some other sources. The goal is then to construct a data-based classification rule $\psi_{n}$, whose conditional error rate

$$
L_{n}\left(\psi_{n}\right)=P\left\{\psi_{n}(\mathbf{Z}) \neq Y \mid \mathcal{D}_{n}\right\}
$$


is in some sense, as small as possible.

Definition 1.2.2 (Data-based Classifier) Let $\mathcal{D}_{n}:=\left\{\left(\mathbf{Z}_{1}, Y_{1}\right), \cdots,\left(\mathbf{Z}_{n}, Y_{n}\right)\right\}$ be a random sample of i.i.d. $\mathcal{R}^{p} \times\{0,1\}$-valued random vectors. Then a data-based classifier $\psi_{n}$ is a mapping of the form

$$
\psi_{n}: \mathcal{R}^{p} \times\left\{\mathcal{R}^{p} \times\{0,1\}\right\}^{n} \rightarrow\{0,1\}
$$

A desirable property for a data-based classifier is consistency which is defined in the next definition.

Definition 1.2.3 (Weak and Strong Consistency) $A$ classification rule $\left\{\psi_{n}, n \geqslant 1\right\}$ is said to be (weakly) consistent if for a certain distribution of $(\mathbf{Z}, Y)$

$$
L_{n}\left(\psi_{n}\right) \stackrel{p}{\rightarrow} L\left(\psi_{B}\right), \text { as } n \rightarrow \infty
$$

or equivalently from Lebesgue Dominated Convergence Theorem

$$
E\left(L_{n}\left(\psi_{n}\right)\right) \rightarrow L\left(\psi_{B}\right) \text {, as } n \rightarrow \infty
$$

A classification rule $\psi_{n}$ is said to be strongly consistent if the foregoing convergence holds almost surely, i.e.,

$$
L_{n}\left(\psi_{n}\right) \stackrel{a . s .}{\rightarrow} L\left(\psi_{B}\right), \text { as } n \rightarrow \infty
$$

If a rule is consistent for all distributions of $(\mathbf{Z}, Y)$, it is said to be universally consistent. A consistent classification rule guarantees that by increasing the sample size $n$, the probability that $L_{n}\left(\psi_{n}\right)$ is within a very small distance of $L\left(\psi_{B}\right)$ gets arbitrarily close to one. Strong consistency means that by using more data $L_{n}\left(\psi_{n}\right)$ gets arbitrarily close to $L\left(\psi_{B}\right)$ for every training sample except for a set of sequences 
that has zero probability altogether. Consistency results for data-based classification rules have been obtained by Van Ryzin (1966) and Glick (1973,1976). Later some other authors e.g., Devroye and Wagner (1980), Krzyzak and Pawlak (1984), Krzyzak (1986) investigated the strong consistency for data-based classifiers. Now the question is: How does one construct a consistent data-based classifier? The next section provides examples of some popular classifiers as well as the conditions under which consistency (weak or strong) holds.

\subsection{Classification Rules}

Classification based on observing complete data is a well-established field of statistics and there are several consistent parametric and nonparametric classifiers in the literature. Selecting the optimal method for classification depends mainly on the type of available information. For example, if the form of the distribution $F_{\mathbf{Z}, Y}$ is known, one uses the data to estimate the parameters of the distribution and constructs a classifier based on one of the parametric methods. But in the case where there is no information available about the distribution $F_{\mathbf{Z}, Y}$, it makes sense to use nonparametric classifiers. In this section we will define some commonly used

classification rules. Some of these rules will be studied in depth later. The book by Devroye, Györfi and Logosi (1996) provides an excellent review of many important classification rules. 


\subsubsection{Parametric Rules}

The Bayes classifier defined in (1.1) can be represented by the conditional distributions of $\mathbf{Z}$ given $Y=0$ and $Y=1$. Note that

$$
\eta_{\boldsymbol{\theta}}(\mathbf{Z})=P\{Y=1 \mid \mathbf{Z}\}=\frac{f_{1}\left(\mathbf{Z}, \boldsymbol{\theta}_{1}\right) p}{f_{1}\left(\mathbf{Z}, \boldsymbol{\theta}_{1}\right) p+f_{0}\left(\mathbf{Z}, \boldsymbol{\theta}_{0}\right)(1-p)},
$$

where

$$
\begin{aligned}
& f_{1}\left(\mathbf{Z}, \boldsymbol{\theta}_{1}\right)=\text { density function for } \mathbf{Z} \text { when } Y=1, \\
& f_{0}\left(\mathbf{Z}, \boldsymbol{\theta}_{0}\right)=\text { density function for } \mathbf{Z} \text { when } Y=0, \\
& \boldsymbol{\theta}=\left(\boldsymbol{\theta}_{0}, \boldsymbol{\theta}_{1}, p\right)=\text { the parameter vector, } \\
& p=P\{Y=1\}=1-P\{Y=0\} .
\end{aligned}
$$

Then, the Bayes classifier is

$$
\psi_{B}(\mathbf{z})= \begin{cases}1 & \text { if } f_{1}\left(\mathbf{z}, \boldsymbol{\theta}_{1}\right) p>f_{0}\left(\mathbf{z}, \boldsymbol{\theta}_{0}\right)(1-p) \\ 0 & \text { otherwise }\end{cases}
$$

Under the parametric approach, the class-conditional densities $\left(f_{1}\left(\mathbf{Z}, \boldsymbol{\theta}_{1}\right), f_{0}\left(\mathbf{Z}, \boldsymbol{\theta}_{0}\right)\right)$ are taken to be known up to a manageable number of parameters. For constructing a data-based classifier one may estimates $\left(\boldsymbol{\theta}_{0}, \boldsymbol{\theta}_{1}, p\right)$ by $\left(\widehat{\boldsymbol{\theta}}_{0}, \widehat{\boldsymbol{\theta}}_{1}, \widehat{p}\right)$ from the data and form the plug-in rule

$$
\psi_{n}(\mathbf{z})= \begin{cases}1 & \text { if } f_{1}\left(\mathbf{z}, \widehat{\boldsymbol{\theta}}_{1}\right) \widehat{p}>f_{0}\left(\mathbf{z}, \widehat{\boldsymbol{\theta}}_{0}\right)(1-\widehat{p}) \\ 0 & \text { otherwise }\end{cases}
$$

The following theorem from Devroye et al. (1996) investigates the basic consistency properties of $\psi_{n}$ based upon continuity considerations. 
Theorem 1.3.1 If $\eta_{\boldsymbol{\theta}}$ as defined in (1.3) is continuous in $\boldsymbol{\theta}$ in the $L_{1}(\mu)$ sense, where $\mu$ is the probability measure of $\mathbf{Z}$, and $\widehat{\boldsymbol{\theta}} \stackrel{p}{\rightarrow} \boldsymbol{\theta}$, then $E\left\{L_{n}\left(\psi_{n}\right)\right\} \rightarrow L\left(\psi_{B}\right)$, where $\psi_{n}$ is the standard plug-in rule defined in (1.4) and $L_{n}\left(\psi_{n}\right)=P\left\{\psi_{n}(\mathbf{Z}) \neq\right.$ $\left.Y \mid \mathcal{D}_{n}\right\}$.

For methods of estimating the parameters see, Devroye et al. (1996). Also McLachlan (2004) has a comprehensive treatment on parametric classification.

\subsubsection{Linear Discriminant Rules}

In linear classification one assumes the classification rule is a linear combination of the components $\mathbf{z}=\left(z_{1}, \cdots, z_{p}\right)^{\prime}$. More precisely, for the feature vector $\mathbf{z} \in \mathcal{R}^{p}$, the linear discriminant rules split the space by a hyperplane and assign a different class to each halfspace, based on the weights associated with each component. More formally, the linear discriminant rule is given by

$$
\psi(\mathbf{z})= \begin{cases}1 & \text { if } a_{0}+\mathbf{a}^{\prime} \mathbf{z}>0 \\ 0 & \text { otherwise }\end{cases}
$$

where $\mathbf{z}=\left(z_{1}, \cdots, z_{p}\right)^{\prime}$ and $a_{0}, \mathbf{a}=\left(a_{1}, \cdots, a_{p}\right)^{\prime}$ are the weights. These weights determine the relative importance of components. The procedure for implementing linear discriminant rules is dependent on the dimension $p$ of $\mathbf{Z}$ and it is quite simple to implement. However, in general, the linear discriminant rules cannot provide error probabilities that are close to the Bayes error probability, but that should not diminish the value of this classifier. Linear classification is at the heart of nearly every successful classification method like tree classifiers, generalized linear classifiers and neural networks. In the case of $\mathcal{R}^{2}$, the linear discriminant rule $\psi$ defines a 
separating line. Fisher's (1936) Linear Discriminant Function is one example of a linear discriminant rule. Note that, linear discriminant rules may be arbitrarily bad even though when the two classes are linearly separable (i.e., $L\left(\psi_{B}\right)=0$ ), as the error probabilities of these types of classifiers may be close to one. The multivariate normal distribution with identical matrix covariances, however, is an exception in which the Bayes rule is a linear discriminant rule. The general multivariate normal density of a random variable $\mathbf{Z}$ is given by

$$
f(\mathbf{z})=\frac{1}{\sqrt{(2 \pi)^{p}|\Sigma|}} e^{-\frac{1}{2}(\mathbf{z}-\boldsymbol{\mu})^{\prime} \Sigma^{-1}(\mathbf{z}-\boldsymbol{\mu})}
$$

where $\boldsymbol{\mu}=E(\mathbf{Z})$ is the mean ( $\boldsymbol{\mu}$ is a $p$-dimensional column vector), and $\Sigma=$ $E\left\{(\mathbf{Z}-\boldsymbol{\mu})(\mathbf{Z}-\boldsymbol{\mu})^{\prime}\right\}$ is the $p \times p$ covariance matrix. Let $f_{0}(\mathbf{z})$ and $f_{1}(\mathbf{z})$ be the conditional densities given $\{Y=0\}$ and $\{Y=1\}$ respectively. In this case, the Bayes rule is given by (1.5) where, by letting $p=P\{Y=1\}$ and $1-p=P\{Y=0\}$,

$$
\begin{aligned}
& \qquad a_{0}=2 \log \left(\frac{p}{1-p}\right)+\boldsymbol{\mu}_{0}^{\prime} \Sigma^{-1} \boldsymbol{\mu}_{0}-\boldsymbol{\mu}_{1}^{\prime} \Sigma^{-1} \boldsymbol{\mu}_{1} \\
& \text { and } \mathbf{a}=\left(a_{1}, \cdots, a_{p}\right)^{\prime}=2\left(\boldsymbol{\mu}_{1}-\boldsymbol{\mu}_{0}\right)^{\prime} \Sigma^{-1} \text {. }
\end{aligned}
$$

Here, it is assumed $\Sigma_{0}=\Sigma_{1}=\Sigma$. In practice though, $p, \boldsymbol{\mu}_{0}, \boldsymbol{\mu}_{1}, \Sigma_{0}, \Sigma_{1}$ are unknown and have to be estimated from the data. Note that, if $\Sigma_{0} \neq \Sigma_{1}$, one has a Quadratic Discrimination Rule.

\subsubsection{Nonparametric Rules}

Parametric approaches are developed by explicit assumptions about underlying distributions. However, there is also a need for classifiers that can be used without any assumptions about the underlying class-conditional distributions. In this sense, they are referred to as nonparametric rules. Fix and Hodges $(1951,1952)$ introduced 
important nonparametric concepts in classification analysis and density estimation. Rosenblatt (1956) and Parzen (1962) proposed nonparametric density estimation, then Nadaraya (1970) and Watson (1964) developed their idea and derived a nonparametric estimation for regression functions which are applied extensively in classification. Many research monographs have been devoted to these topics, including those by Tapia and Thompson (1978), Prakaso Rao (1983), Silverman (1986) and Devroye and Györfi (1985). Here, we will define some nonparametric classification rules that are commonly used in the literature and will be referred to in the subsequent chapters, when we develop our proposed methods. In general, these rules are based on the local average estimator.

Definition 1.3.1 (Local Average Estimator) An estimate of $\eta_{B}$ of the form

$$
\eta_{n}(\mathbf{z})=\sum_{i=1}^{n} I\left\{Y_{i}=1\right\} \omega_{n i}(\mathbf{z})=\sum_{i=1}^{n} Y_{i} \omega_{n i}(\mathbf{z})
$$

is a local average estimator. Here $\omega_{n i}(\mathbf{z})=\omega_{n}\left(\mathbf{z}, \mathbf{Z}_{1}, \cdots, \mathbf{Z}_{n}\right)$ weights those values of $i$ for which $\mathbf{Z}_{i}$ is close to $\mathbf{z}$ more heavily than those values of $i$ for which $\mathbf{Z}_{i}$ is far from $\mathbf{z} . \omega_{n i}$ is said to be a probability weight function if it is nonnegative and

$$
\sum_{i=1}^{n} \omega_{n i}(\mathbf{z})=1
$$

The classification rule defined as

$$
\psi_{n}(\mathbf{z})= \begin{cases}1 & \text { if } \sum_{i=1}^{n} \omega_{n i}(\mathbf{z}) I\left\{Y_{i}=1\right\}>\sum_{i=1}^{n} \omega_{n i}(\mathbf{z}) I\left\{Y_{i}=0\right\} \\ 0 & \text { otherwise, }\end{cases}
$$

is called a local weighted majority vote.

A general theorem by Stone (1977) allows us to deduce the universal consistency of the group of classifiers defined above. 
Theorem 1.3.2 (Stone (1977)) Let $\left\{\omega_{n}\right\}$ be a sequence of probability weights. The corresponding regression function is consistent if and only if the following three conditions hold: there is a $c \geqslant 1$ such that, for every nonnegative Borel function $f$ on $\mathcal{R}^{p}, E \sum_{i=1}^{n} \omega_{n_{i}}(\mathbf{Z}) f\left(\mathbf{Z}_{i}\right) \leqslant c E f(\mathbf{Z})$ for all $n \geqslant 1 ; \sum_{i=1}^{n} \omega_{n_{i}}(\mathbf{Z}) I\left\{\left\|\mathbf{Z}_{i}-\mathbf{Z}\right\|>a\right\} \rightarrow 0$ in probability for all $a>0$; and $\max _{i} \omega_{n_{i}}(\mathbf{Z}) \rightarrow 0$ in probability.

Examples of such rules include the partitioning, histogram, kernel, and nearest neighbour rules. These rules will be defined below.

\section{Partitioning Rules}

Many important classification rules partition $\mathcal{R}^{p}$ into disjoint cells $A_{1}, A_{2}, \cdots$ and classify in each cell according to the majority vote among the labels of the $\mathbf{Z}_{i}$ 's falling in the cell containing $\mathbf{Z}$. Formally,

$$
\psi_{n}(\mathbf{z})= \begin{cases}1 & \text { if } \sum_{i=1}^{n} I\left\{\mathbf{Z}_{i} \in A(\mathbf{z})\right\} I\left\{Y_{i}=1\right\}>\sum_{i=1}^{n} I\left\{\mathbf{Z}_{i} \in A(\mathbf{z})\right\} I\left\{Y_{i}=0\right\} \\ 0 & \text { otherwise }\end{cases}
$$

where $A(\mathbf{z})$ denotes the cell containing $\mathbf{z}$. The next theorem is a general consistency result for such partitioning rules (see, for example Devroye et al. (1996), Chapter $6)$ :

Theorem 1.3.3 Consider the partitioning classification rule as defined in (1.6).

Then $E\left\{L_{n}\left(\psi_{n}\right)\right\} \rightarrow L\left(\psi_{B}\right)$ if

(i) $\operatorname{diam}(A(\mathbf{Z})) \rightarrow 0$ in probability,

(ii) $N(\mathbf{Z}) \rightarrow \infty$ in probability,

where

$$
\operatorname{diam}(A)=\sup _{\mathbf{z}_{1}, \mathbf{z}_{2} \in A}\left\|\mathbf{z}_{1}-\mathbf{z}_{2}\right\|, \quad \text { and } \quad N(\mathbf{z})=\sum_{i=1}^{n} I\left\{\mathbf{Z}_{i} \in A(\mathbf{z})\right\}
$$




\section{The Regular Histogram Rule}

This rule partitions $\mathcal{R}^{p}$ into cubes of the same size, then makes a decision according to the majority vote among the $Y_{i}$ 's for which the corresponding $\mathbf{Z}_{i}$ fall in the same cube as $\mathbf{Z}$. (recall that $\left(\mathbf{Z}_{i}, Y_{i}\right), i=1, \cdots, n$ are i.i.d. $\mathcal{R}^{p} \times\{0,1\}$-valued random vectors). Now, consider $\mathcal{P}_{n}=\left\{A_{n_{1}}, A_{n_{2}}, \cdots\right\}$ as a partition of $\mathcal{R}^{p}$ into cubes of size $h_{n}>0$, that is, into sets of the type $\prod_{i=1}^{p}\left[k_{i} h_{n},\left(k_{i}+1\right) h_{n}\right]$, where the $k_{i}$ 's are integers, then the histogram rule is defined by

$$
\psi_{n}(\mathbf{z})=\left\{\begin{array}{cc}
1 & \text { if } \quad \sum_{i=1}^{n} I\left\{\mathbf{Z}_{i} \in A_{n}(\mathbf{z})\right\} I\left\{Y_{i}=1\right\}> \\
& \quad \sum_{i=1}^{n} I\left\{\mathbf{Z}_{i} \in A_{n}(\mathbf{z})\right\} I\left\{Y_{i}=0\right\} \\
0 & \text { otherwise, }
\end{array}\right.
$$

where for every $\mathbf{z} \in \mathcal{R}^{p}, A_{n}(\mathbf{z})=A_{n i}$ if $\mathbf{z} \in A_{n i}$. This rule is strongly universally consistent based on following theorem which is essentially due to Devroye and Györfi (1983).

Theorem 1.3.4 Assume that the sequence of partitions $\left\{\mathcal{P}_{n}\right\}$ satisfies the following two conditions as $n \rightarrow \infty$

$$
h_{n} \rightarrow 0 \quad \text { and } \quad n h_{n}^{p} \rightarrow \infty
$$

For any distribution of $(\mathbf{Z}, Y)$, and for every $\epsilon>0$ there is an integer $n_{0}$ such that for $n>n_{0}$, for the error probability $L_{n}\left(\psi_{n}\right)$ of the histogram rule

$$
P\left\{L_{n}\left(\psi_{n}\right)-L\left(\psi_{B}\right)>\epsilon\right\} \leqslant 2 e^{-n \epsilon^{2} / 32}
$$

Thus, the histogram rule is strongly universally consistent (due to the Borel-Cantelli lemma). 


\section{Kernel Rules}

The histogram rule in application, is less accurate at the borders of the cells of the partition than in the middle of the cells. To overcome this problem, Rosenblatt (1956) used the idea of the moving average of time series analysis and introduced moving window instead of having a fixed cell structure independent of the position of $\mathbf{Z}$. The moving window rule takes the data points within a certain distance of the point to be classified, then classifies that point according to a majority vote. Formally, let $h_{n}$ be a positive number and define the moving window rule (also known as a naïve kernel) as

$$
\psi_{n}(\mathbf{z})= \begin{cases}1 & \text { if } \sum_{i=1}^{n} I\left\{Y_{i}=1, \mathbf{Z}_{i} \in S_{\mathbf{z}, h_{n}}\right\}>\sum_{i=1}^{n} I\left\{Y_{i}=0, \mathbf{Z}_{i} \in S_{\mathbf{z}, h_{n}}\right\} \\ 0 & \text { otherwise }\end{cases}
$$

where $S_{\mathbf{z}, h_{n}}$ denotes a closed ball of radius $h_{n}>0$ centered at $\mathbf{z}$.

It is possible to make the above rule smoother by giving more weight to closer points than further points. Let $\mathcal{K}: \mathcal{R}^{p} \rightarrow \mathcal{R}$ be a kernel function, where $\mathcal{K} \geqslant 0$ and $\mathcal{K}(\mathbf{t})$ is monotone decreasing in $\|\mathbf{t}\|$. Then, the kernel classification rule, which is a generalization of the moving window rule, is given by

$$
\psi_{n}(\mathbf{z})= \begin{cases}1 & \text { if } \sum_{i=1}^{n} I\left\{Y_{i}=1\right\} \mathcal{K}\left(\frac{\mathbf{z}-\mathbf{Z}_{i}}{h_{n}}\right)>\sum_{i=1}^{n} I\left\{Y_{i}=0\right\} \mathcal{K}\left(\frac{\mathbf{z}-\mathbf{Z}_{i}}{h_{n}}\right) \\ 0 & \text { otherwise }\end{cases}
$$

where $h_{n}$ is a smoothing factor or bandwidth depending only on $n$. Two popular kernels that we will use later are the Gaussian kernel, $\mathcal{K}(\mathbf{z})=e^{-\|\mathbf{z}\|^{2}}$, and the Cauchy kernel, $\mathcal{K}(\mathbf{z})=1 /\left(1+\|\mathbf{z}\|^{p+1}\right)$ where $\|\cdot\|$ denotes the Euclidean distance. Devroye and Krzyzak (1989) proved the strong universal consistency of the kernel classifier for a large class of kernel functions called regular kernels. 
Definition 1.3.2 (Regular Kernel) The kernel $\mathcal{K}$ is called regular if $\mathcal{K} \geqslant 0$, and there is a ball $S_{0, r}$ of radius $r>0$ centered at the origin, and constant $B>0$ such that $\mathcal{K}(\mathbf{z}) \geqslant B I_{S_{0, r}}$ and $\int \sup _{\mathbf{w} \in \mathbf{z}+S_{0, r}} \mathcal{K}(\mathbf{w}) d \mathbf{z}<\infty$

Theorem 1.3.5 Suppose $\mathcal{K}$ is a regular kernel. If $h_{n} \rightarrow 0$ and $n h_{n}^{p} \rightarrow \infty$, as $n \rightarrow \infty$, then for any distribution of $(\mathbf{Z}, Y)$, and for every $\epsilon>0$ there is an integer $n_{0}$ such that for $n>n_{0}$,

$$
P\left\{L_{n}\left(\psi_{n}\right)-L\left(\psi_{B}\right)>\epsilon\right\} \leqslant 2 e^{-n \epsilon^{2} / 32 \rho^{2}}
$$

where the constant $\rho$ depends on the kernel $\mathcal{K}$ and the dimension only. Thus, the kernel classification rule is strongly universally consistent.

\section{Nearest Neighbour Rules}

Nearest neighbour procedures were first studied in the context of nonparametric classification by Fix and Hodges (1951). To define these rules, let $\left\|\mathbf{Z}-\mathbf{Z}_{i}\right\|$ be the Euclidean distance between $\mathbf{Z}_{i}$ and $\mathbf{Z}$. $\mathbf{Z}_{i}$ is said to be the $k^{\text {th }}$ nearest neighbour of $\mathbf{Z}$ if the distance $\left\|\mathbf{Z}-\mathbf{Z}_{i}\right\|$ is the $k^{\text {th }}$ smallest among $\left\|\mathbf{Z}-\mathbf{Z}_{1}\right\|, \cdots\left\|\mathbf{Z}-\mathbf{Z}_{n}\right\|$. In the case of a distance tie, the candidate with the smaller index is said to be closer to $\mathbf{Z}$. Formally, the $k$-NN ( $k$-nearest neighbour ) rule, proposed by Fix and Hodges (1951), is defined by

$$
\psi_{n}(\mathbf{z})= \begin{cases}1 & \text { if } \sum_{i=1}^{n} \omega_{n i} I\left\{Y_{i}=1\right\}>\sum_{i=1}^{n} \omega_{n i} I\left\{Y_{i}=0\right\} \\ 0 & \text { otherwise }\end{cases}
$$

where $\omega_{n i}=\frac{1}{k}$ if $\mathbf{Z}_{i}$ is among the $k$ nearest neighbours of $\mathbf{Z}$ and $\omega_{n i}=0$ otherwise. Note that the $k$-NN rule is based on a majority vote among the labels of the $k$ 
nearest neighbours of $\mathbf{z}$. It is convenient to let $k$ be odd in order to avoid a voting tie. By an application of Stone's theorem (1977), one can show that the $k$-NN rule is weakly universally consistent if $k$ is allowed to grow with $n$ in such a way that $\frac{k}{n} \rightarrow 0$

Theorem 1.3.6 (Stone (1977)) If $k \rightarrow \infty$ and $\frac{k}{n} \rightarrow 0$, then for all distributions $E\left\{L_{n}\left(\psi_{n}\right)\right\} \rightarrow L\left(\psi_{B}\right)$

The issue of tie breaking becomes important when one is concerned with strong consistency of the $k$-NN classification rule. Devroye and Györfi (1985) and Zhao (1987), proved that the $k$-NN rule is strongly consistent under the assumption that $\mathbf{Z}$ has a density function. This assumption is needed to avoid the problem of handling distance ties.

\subsection{Selecting Classifiers}

In this section, we deal with discrimination rules that are picked empirically (i.e., using the data $\mathcal{D}_{n}$ ) from a certain class of classifiers. To explain and motivate this approach, we first state the following well-known simple result (see, e.g., Van Ryzin (1966), Glick (1973), Devroye and Györfi (1985)).

Theorem 1.4.1 Let $\eta(\mathbf{z})$ be a nonnegative function that approximate $\eta_{B}(\mathbf{z})$. Define the plug-in decision $\psi$ by

$$
\psi(\mathbf{z})= \begin{cases}1 & \text { if } \eta(\mathbf{z})>\frac{1}{2} \\ 0 & \text { otherwise }\end{cases}
$$


Then

$$
0 \leqslant P\{\psi(\mathbf{Z}) \neq Y\}-P\left\{\psi_{B}(\mathbf{Z}) \neq Y\right\} \leqslant 2 E\left|\eta_{B}(\mathbf{Z})-\eta(\mathbf{Z})\right|,
$$

where $\psi_{B}$ defined in (1.1).

The above theorem states that if $\eta(\mathbf{z})$ is close to $\eta_{B}(\mathbf{z})$ in the $L_{1}$-sense, then the error probability of decision $\psi$ is near the optimal decision $\psi_{B}$. In many cases, $\eta_{B}$ is estimated from the training data $\mathcal{D}_{n}$ by some function $\eta_{n}(\mathbf{z})=\eta_{n}\left(\mathbf{z}, \mathcal{D}_{n}\right)$. Note that, the error probability $L_{n}\left(\psi_{n}\right)=P\left\{\psi_{n}(\mathbf{Z}) \neq Y \mid \mathcal{D}_{n}\right\}$ of the plug-in rule

$$
\psi_{n}(\mathbf{z})= \begin{cases}1 & \text { if } \eta_{n}(\mathbf{z})>\frac{1}{2} \\ 0 & \text { otherwise }\end{cases}
$$

is a random variable. Then a simple corollary of Theorem 1.4.1 is as follows (see Devroye et al. (1996), Chapter 6).

Corollary 1.4.2 The error probability of the classifier $\psi_{n}(\mathbf{z})$ defined above satisfies

$$
\begin{aligned}
L_{n}\left(\psi_{n}\right)-L\left(\psi_{B}\right) & \leqslant 2 E\left\{\left|\eta_{B}(\mathbf{Z})-\eta_{n}(\mathbf{Z})\right| \mathcal{D}_{n}\right\} \\
& \left.\leqslant 2 \sqrt{E\left\{\left(\eta_{B}(\mathbf{Z})-\eta_{n}(\mathbf{Z})\right)^{2} \mid \mathcal{D}_{n}\right\}} \quad \text { (Cauchy-Schwarz inequality }\right) .
\end{aligned}
$$

Thus estimates $\eta_{n}$ with small $L_{2}$ error automatically lead to estimates $\psi_{n}$ with small misclassification probability. So, one often constructs estimates by minimizing the empirical $L_{2}$ risk $\frac{1}{n} \sum_{i=1}^{n}\left(\eta_{n}\left(\mathbf{Z}_{i}\right)-Y_{i}\right)^{2}$. More precisely, let $\mathcal{M}$ be a class of candidate functions $\eta: \mathcal{R}^{p} \rightarrow[0,1]$ that contains $\eta_{B}$. The least squares estimator of $\eta_{B}$ is given by

$$
\eta_{n}=\underset{\eta \in \mathcal{M}}{\operatorname{argmin}} \frac{1}{n} \sum_{i=1}^{n}\left(Y_{i}-\eta\left(\mathbf{Z}_{i}\right)\right)^{2}
$$


According to the Corollary 1.4.2

$$
\begin{aligned}
L_{n}\left(\psi_{n}\right)-L\left(\psi_{B}\right) & \leqslant 2 \sqrt{E\left\{\left(\eta_{n}(\mathbf{Z})-\eta_{B}(\mathbf{Z})\right)^{2} \mid \mathcal{D}_{n}\right\}} \\
& =2 \sqrt{E\left\{\left(\eta_{n}(\mathbf{Z})-Y\right)^{2} \mid \mathcal{D}_{n}\right\}-E\left\{\left(\eta_{B}(\mathbf{Z})-Y\right)^{2}\right\}} \\
& =2 \sqrt{E\left\{\left(\eta_{n}(\mathbf{Z})-Y\right)^{2} \mid \mathcal{D}_{n}\right\}-\inf _{\eta \in \mathcal{M}} E\left\{(\eta(\mathbf{Z})-Y)^{2}\right\}} \\
& =2 \sqrt{\sup _{\eta \in \mathcal{M}}\left[E\left\{\left(\eta_{n}(\mathbf{Z})-Y\right)^{2} \mid \mathcal{D}_{n}\right\}-E\left\{(\eta(\mathbf{Z})-Y)^{2}\right\}\right]}
\end{aligned}
$$

where we have used the elementary argument that

$$
\begin{aligned}
E\left\{\left(\eta_{n}(\mathbf{Z})-Y\right)^{2} \mid \mathcal{D}_{n}\right\} & =E\left\{\left(\eta_{n}(\mathbf{Z})-\eta_{B}(\mathbf{Z})+\eta_{B}(\mathbf{Z})-Y\right)^{2} \mid \mathcal{D}_{n}\right\} \\
& =E\left\{\left(\eta_{n}(\mathbf{Z})-\eta_{B}(\mathbf{Z})\right)^{2} \mid \mathcal{D}_{n}\right\}+E\left(\eta_{B}(\mathbf{Z})-Y\right)^{2}
\end{aligned}
$$

Note that

$$
\begin{aligned}
\sup _{\eta \in \mathcal{M}}\left[E\left\{\left(\eta_{n}(\mathbf{Z})-Y\right)^{2} \mid \mathcal{D}_{n}\right\}-E\left\{(\eta(\mathbf{Z})-Y)^{2}\right\}\right] \\
=\sup _{\eta \in \mathcal{M}}\left[E\left\{\left(\eta_{n}(\mathbf{Z})-Y\right)^{2} \mid \mathcal{D}_{n}\right\}-\frac{1}{n} \sum_{i=1}^{n}\left(\eta_{n}\left(\mathbf{Z}_{i}\right)-Y_{i}\right)^{2}+\frac{1}{n} \sum_{i=1}^{n}\left(\eta_{n}\left(\mathbf{Z}_{i}\right)-Y_{i}\right)^{2}\right. \\
\left.\quad-\frac{1}{n} \sum_{i=1}^{n}\left(\eta\left(\mathbf{Z}_{i}\right)-Y_{i}\right)^{2}+\frac{1}{n} \sum_{i=1}^{n}\left(\eta\left(\mathbf{Z}_{i}\right)-Y_{i}\right)^{2}-E\left\{(\eta(\mathbf{Z})-Y)^{2}\right\}\right] \\
\leqslant 2 \sup _{\eta \in \mathcal{M}}\left|\frac{1}{n} \sum_{i=1}^{n}\left(\eta\left(\mathbf{Z}_{i}\right)-Y_{i}\right)^{2}-E\left\{(\eta(\mathbf{Z})-Y)^{2}\right\}\right| .
\end{aligned}
$$

In the above inequality, we have used the fact that $\frac{1}{n} \sum_{i=1}^{n}\left(\eta_{n}\left(\mathbf{Z}_{i}\right)-Y_{i}\right)^{2}-$ $\frac{1}{n} \sum_{i=1}^{n}\left(\eta\left(\mathbf{Z}_{i}\right)-Y_{i}\right)^{2} \leqslant 0$, as $\eta_{n}$ is selected by minimizing the empirical squared error. Finally

$$
L_{n}\left(\psi_{n}\right)-L\left(\psi_{B}\right) \leqslant 2 \sqrt{2 \sup _{\eta \in \mathcal{M}}\left|\frac{1}{n} \sum_{i=1}^{n}\left(\eta\left(\mathbf{Z}_{i}\right)-Y_{i}\right)^{2}-E\left\{(\eta(\mathbf{Z})-Y)^{2}\right\}\right|} .
$$


Thus, the least-squares method is consistent if the supremum above converges to zero. Therefore, we are interested in bounding the distance between an average and its expectation uniformly over a set of functions. In the special case where the class $\mathcal{M}$ is finite, one can use the union bound along with Hoeffding's inequality to conclude

$$
\begin{aligned}
& P\left\{L_{n}\left(\psi_{n}\right)-L\left(\psi_{B}\right)>\epsilon\right\} \\
& \leqslant P\left\{\sup _{\eta \in \mathcal{M}}\left|\frac{1}{n} \sum_{i=1}^{n}\left(\eta\left(\mathbf{Z}_{i}\right)-Y_{i}\right)^{2}-E\left\{(\eta(\mathbf{Z})-Y)^{2}\right\}\right|>\frac{\epsilon^{2}}{8}\right\} \\
& \leqslant 2|\mathcal{M}| e^{-n \epsilon^{4} / 32}
\end{aligned}
$$

where $|\mathcal{M}|$ is cardinality of $\mathcal{M}$. For finite classes $\mathcal{M}, \psi_{n}$ is strongly Bayes consistent by the Borel-Cantelli lemma. However, in the realistic case where the class $\mathcal{M}$ contains infinitely many functions, the bound in (1.9) is not summable and the problem becomes nontrivial. The most powerful weapons to attack these problems are to find covers for the class $\mathcal{M}$ and then use Dudley-Pollard-type theorems; see Dudley (1978) and Pollard (1984). For the sake of readability, we first present the following definitions:

Definition 1.4.1 ( $\epsilon$-Cover W.R.T Supnorm) Let $\epsilon>0$ and let $\mathcal{G}$ be $a$ set of functions $g: \mathcal{R}^{p} \rightarrow \mathcal{R}$. Every finite collection of functions $\mathcal{G}_{\epsilon}=$ $\left\{g_{1}, \cdots, g_{N_{(\epsilon)}}: \mathcal{R}^{p} \rightarrow \mathcal{R}\right\}$ with the property that for every $g \in \mathcal{G}$, there is a $g^{*} \in \mathcal{G}_{\epsilon}$ such that

$$
\left\|g-g^{*}\right\|:=\sup _{\mathbf{z}}\left|g(\mathbf{z})-g^{*}(\mathbf{z})\right|<\epsilon
$$

is called an $\epsilon$-cover of $\mathcal{G}$ with respect $\|\cdot\| \cdot \|_{\infty}$. Also, the class $\mathcal{G}$ with this property is called totally bounded. 
Definition 1.4.2 ( $\epsilon$-Covering Number W.R.T Supnorm) Let $\epsilon>0$ and let $\mathcal{G}$ be a set of functions $g: \mathcal{R}^{p} \rightarrow \mathcal{R}$. Let $\mathcal{N}\left(\epsilon, \mathcal{G},\|\cdot\|_{\infty}\right)$ be the size of the smallest $\epsilon$-cover of $\mathcal{G}$ w.r.t. $\|\cdot\|_{\infty}$. Take $\mathcal{N}\left(\epsilon, \mathcal{G},\|\cdot\|_{\infty}\right)=\infty$ if no finite $\epsilon$-cover exists. Then $\mathcal{N}\left(\epsilon, \mathcal{G},\|\cdot\|_{\infty}\right)$ is called the $\epsilon$-covering number of $\mathcal{G}$ w.r.t. $\|\cdot\|_{\infty}$ and will be abbreviated to $\mathcal{N}_{\infty}(\epsilon, \mathcal{G})$.

The following lemma is based on fixed supremum norm covers as defined above (see, Györfi et al. (2002)).

Lemma 1.4.3 Let $\mathcal{G}_{n}$ be a set of functions $g: \mathcal{R}^{p} \rightarrow[0, B]$. Then for every $\epsilon>0$ and all $n \geqslant 1$

$$
P\left\{\sup _{g \in \mathcal{G}_{n}}\left|\frac{1}{n} \sum_{i=1}^{n} g\left(\mathbf{Z}_{i}\right)-E\{g(\mathbf{Z})\}\right|>\epsilon\right\} \leqslant 2 \mathcal{N}_{\infty}\left(\epsilon / 3, \mathcal{G}_{n}\right) e^{-\frac{2 n \epsilon^{2}}{9 B^{2}}} .
$$

Furthermore, if

$$
\sum_{n=1}^{\infty} \mathcal{N}_{\infty}\left(\epsilon / 3, \mathcal{G}_{n}\right) e^{-\frac{2 n \epsilon^{2}}{9 B^{2}}}<\infty
$$

for all $\epsilon>0$, then

$$
\sup _{g \in \mathcal{G}_{n}}\left|\frac{1}{n} \sum_{i=1}^{n} g\left(\mathbf{Z}_{i}\right)-E\{g(\mathbf{Z})\}\right| \stackrel{\text { a.s. }}{\rightarrow} 0 \quad \text { as } n \rightarrow \infty .
$$

The following inequality is a result of Lemma 1.4.3, by considering $g(\mathbf{Z}, Y)=$ $\left(\eta\left(\mathbf{Z}_{i}\right)-Y_{i}\right)^{2}$

$$
\begin{aligned}
& P\left\{L_{n}\left(\psi_{n}\right)-L\left(\psi_{B}\right)>\epsilon\right\} \\
& \leqslant P\left\{\sup _{\eta \in \mathcal{M}}\left|\frac{1}{n} \sum_{i=1}^{n}\left(\eta\left(\mathbf{Z}_{i}\right)-Y_{i}\right)^{2}-E\left\{(\eta(\mathbf{Z})-Y)^{2}\right\}\right|>\frac{\epsilon^{2}}{8}\right\} \\
& \leqslant 2 \mathcal{N}_{\infty}\left(\epsilon^{2} / 24, \mathcal{M}\right) e^{-n \epsilon^{4} / 288},
\end{aligned}
$$


where $\mathcal{M}$ is a totally bounded class of functions $\eta: \mathcal{R}^{p} \rightarrow[0,1]$, containing $\eta_{B}$. Here $\psi_{n}$ is strongly consistent by an application of the Borel-Cantelli lemma.

Supremum norm covers are often too large and one is typically interested in carrying out the analysis based on a weaker condition like the $L_{1}$-norm covering which is defined as follows.

Definition 1.4.3 ( $\epsilon$-Cover W.R.T $L_{1}$-Norm) Let $\epsilon>0$ and let $\mathcal{G}$ be a set of functions $g: \mathcal{R}^{p} \rightarrow \mathcal{R}$, and $\nu$ be a probability measure on $\mathcal{R}^{p}$. For a function $f: \mathcal{R}^{p} \rightarrow \mathcal{R}$

$$
\|f\|_{L_{1}(\nu)}:=\int|f(\mathbf{z})| d \nu
$$

Every finite collection of functions $\mathcal{G}_{\epsilon}=\left\{g_{1}, \cdots, g_{N_{(\epsilon)}}: \mathcal{R}^{p} \rightarrow \mathcal{R}\right\}$ with the property that for every $g \in \mathcal{G}$, there is a $g^{*} \in \mathcal{G}_{\epsilon}$ such that

$$
\left\|g-g^{*}\right\|_{L_{1}(\nu)}<\epsilon
$$

is called an $\epsilon$-cover of $\mathcal{G}$ with respect to $\|\cdot\|_{L_{1}(\nu)}$.

Definition 1.4.4 ( $\epsilon$-Covering Number W.R.T $L_{1}$-Norm) Let $\mathcal{N}_{1}\left(\epsilon, \mathcal{G},\|\cdot\|_{L_{1}(\nu)}\right)$ be the size of the smallest $\epsilon$-cover of $\mathcal{G}$ w.r.t. $\|\cdot\|_{L_{1}(\nu)}$. Take $\mathcal{N}\left(\epsilon, \mathcal{G},\|\cdot\|_{L_{1}(\nu)}\right)=\infty$ if no finite $\epsilon$-cover exists. Then $\mathcal{N}_{1}\left(\epsilon, \mathcal{G},\|\cdot\|_{L_{1}(\nu)}\right)$ is called an $\epsilon$-covering number of $\mathcal{G}$ w.r.t. $\|\cdot\|_{L_{1}(\nu)}$.

The above definition can of course be stated for $L_{p}$-norms, $p \geqslant 1$, in general. Since in practice the distribution of $\mathbf{Z}$ is unknown, one may be interested in using the empirical $L_{1}$-norm instead. 
Definition 1.4.5 ( $\epsilon$-Cover W.R.T Empirical $L_{1}$-Norm) Let $\left(\mathbf{z}_{i}\right)_{i=1}^{n}=$ $\left(\mathbf{z}_{1}, \cdots \mathbf{z}_{n}\right)$ be $n$ fixed points in $\mathcal{R}^{p}$. Let $\nu_{n}$ be the corresponding empirical measure, i.e.,

$$
\nu_{n}(A)=\frac{1}{n} \sum_{i=1}^{n} I_{(A)}\left(\mathbf{z}_{i}\right) \quad\left(A \subseteq \mathcal{R}^{p}\right) .
$$

Then, for a function $f: \mathcal{R}^{p} \rightarrow \mathcal{R}$

$$
\|f\|_{L_{1}\left(\nu_{n}\right)}=\frac{1}{n} \sum_{i=1}^{n}\left|f\left(\mathbf{z}_{i}\right)\right| .
$$

Let $\epsilon>0$ and let $\mathcal{G}$ be a set of functions $g: \mathcal{R}^{p} \rightarrow \mathcal{R}$. Every finite collection of functions $\mathcal{G}_{\epsilon}=\left\{g_{1}, \cdots, g_{N_{(\epsilon)}}: \mathcal{R}^{p} \rightarrow \mathcal{R}\right\}$ with the property that for every $g \in \mathcal{G}$, there is a $g^{*} \in \mathcal{G}_{\epsilon}$ such that

$$
\left\|g-g^{*}\right\|_{L_{1}\left(\nu_{n}\right)}=\frac{1}{n} \sum_{i=1}^{n}\left|g\left(\mathbf{z}_{i}\right)-g^{*}\left(\mathbf{z}_{i}\right)\right|<\epsilon
$$

is an $\epsilon$-cover of $\mathcal{G}$ with respect to $\|\cdot\|_{L_{1}\left(\nu_{n}\right)}$. Here, $\mathcal{G}_{\epsilon}$ is called an $L_{1} \epsilon$-cover of $\mathcal{G}$ on $\left(\mathbf{z}_{i}\right)_{i=1}^{n}$

Definition 1.4.6 ( $\epsilon$-Covering Number W.R.T Empirical $L_{1}$-Norm) Let $\mathcal{N}_{1}\left(\epsilon, \mathcal{G},\left(\mathbf{z}_{i}\right)_{i=1}^{n}\right)$ be the size of the smallest $\epsilon$-cover of $\mathcal{G}$ w.r.t. $\|\cdot\|_{L_{1}\left(\nu_{n}\right)}$. Take $\mathcal{N}_{1}\left(\epsilon, \mathcal{G},\left(\mathbf{z}_{i}\right)_{i=1}^{n}\right)=\infty$ if no finite $\epsilon$-cover exists. Then $\mathcal{N}_{1}\left(\epsilon, \mathcal{G},\left(\mathbf{z}_{i}\right)_{i=1}^{n}\right)$ is called an $\epsilon$-covering number of $\mathcal{G}$ w.r.t. $\|\cdot\|_{L_{1}\left(\nu_{n}\right)}$.

Note that, if $\left(\mathbf{Z}_{i}\right)_{i=1}^{n}=\left(\mathbf{Z}_{1}, \cdots, \mathbf{Z}_{n}\right)$ is a sequence of i.i.d. random variables, then $\mathcal{N}_{1}\left(\epsilon, \mathcal{G},\left(\mathbf{Z}_{i}\right)_{i=1}^{n}\right)$ is a random variable, whose expected value plays a central role in our problem. The following result will be used extensively in the subsequent chapters: 
Theorem 1.4.4 (Pollard (1984)) Let $\mathcal{G}$ be a set of functions $g: \mathcal{R}^{p} \rightarrow[0, B]$. For any $n$, and any $\epsilon>0$,

$$
P\left\{\sup _{g \in \mathcal{G}}\left|\frac{1}{n} \sum_{i=1}^{n} g\left(\mathbf{Z}_{i}\right)-E\{g(\mathbf{Z})\}\right|>\epsilon\right\} \leqslant 8 E\left\{\mathcal{N}_{1}\left(\epsilon / 8, \mathcal{G},\left(\mathbf{Z}_{i}\right)_{i=1}^{n}\right)\right\} e^{-n \epsilon^{2} /\left(128 B^{2}\right)} .
$$

This theorem is proved in several steps, following the ideas of Dudley (1978), and Pollard (1984). See also, van der Vaart and Wellner (1996), van de Geer (2000).

We return to the minimization of the empirical squared error. Let $\mathcal{M}$ be a class of functions $\eta: \mathcal{R}^{p} \rightarrow[0,1]$, containing $\eta_{B}$. The empirical squared error

$$
\frac{1}{n} \sum_{i=1}^{n}\left(\eta\left(\mathbf{Z}_{i}\right)-Y_{i}\right)^{2}
$$

is minimized over $\eta \in \mathcal{M}$, to obtain the estimate $\eta_{n}$. The next result shows the empirical squared error minimization is consistent under general conditions (see, Devroye et al. (1996)).

Theorem 1.4.5 Let $\mathcal{M}$ be a totally bounded class of functions $\eta: \mathcal{R}^{p} \rightarrow[0,1]$, containing $\eta_{B}$. Then the classification rule obtained by minimizing the empirical squared error over $\mathcal{M}$ is strongly consistent, i.e.,

$$
\lim _{n \rightarrow \infty} L_{n}\left(\psi_{n}\right)=L\left(\psi_{B}\right) \quad \text { w.p.1, }
$$

where $L_{n}\left(\psi_{n}\right)=P\left\{\psi_{n}(\mathbf{Z}) \neq Y \mid \mathcal{D}_{n}\right\}$.

The proof follows from (1.9) and Theorem 1.4.4 by considering $g(\mathbf{Z}, Y)=(\eta(\mathbf{Z})-$ $Y)^{2}$.

This research aims to find classification rules in the presence of missing covariates. We have explained some results related to classification above. The next section 
explains some basic definitions related to missing data, followed by historical approaches to missing value problems.

\subsection{Missing Covariates}

Standard statistical methods have been developed to analyze complete data, however, in practice, often not all covariates can be measured for all units. For example, respondents in a household survey may refuse to report income. Statistical analysis with missing data has been noted in the literature for more than 70 years; Wilks (1932) initiated a study on the MLE for multivariate normal models with fragmentary data (a set of data with small incomplete parts). Thereafter extensive discussions on this topic continued, notably the work by Anderson (1957) who introduced factor likelihoods for the normal distribution. Later, EM algorithm, a technique to find maximum likelihood estimates from incomplete data, was introduced by Dempster, Laird, and Rubin (1977). A useful reference for general parametric statistical inferences with missing data was provided by Little and Rubin (2002). Nonparametric methods have also been proposed for missing data problems, for example, Cheng (1994) proposed using the kernel conditional mean estimator to impute the missing values. Wang and Rao (2002) developed an empirical likelihood inference for the mean parameter with missing responses where the missing values were imputed with a kernel estimator of the conditional mean. In survey statistics, Kim and Fuller (2004) proposed the fractional hot deck imputation method, in which multiple values are drawn from the same imputation cell as the missing observation, and a weight is assigned to each imputed value.

Missing data mechanisms are crucial since the properties of missing data methods 
depend very strongly on the nature of the dependencies of the missing values and the underlying values of the variables in the data set. In general there are 3 important missing mechanisms as follows:

Definition 1.5.1 (Missing Completely At Random (MCAR)) If missingness does not depend on the values of data $\mathbf{Z}$, missing or observed, that is if

$$
P\{\delta=1 \mid \mathbf{Z}\}=P\{\delta=1\} \quad \text { for all } \mathbf{Z}
$$

the data are called missing completely at random. Here $\delta$ is missing indicator.

The MCAR assumption is very strong, because it requires that the completely observed data be a random subsample of the original sample and so is often unreasonable in practice.

Definition 1.5.2 (Missing At Random (MAR)) If missingness depends only on the components that are observed, and not on the components that are missing, that is,

$$
P\{\delta=1 \mid \mathbf{Z}\}=P\{\delta=1 \mid \mathbf{X}, \mathbf{V}\}=P\{\delta=1 \mid \mathbf{X}\} \quad \text { where } \mathbf{V} \text { is missing }
$$

then the missing data mechanism is called missing at random.

The MAR assumption is much weaker than MCAR, as it allows the missing data to possibly depend on the observed data and may be reasonable for many practical problems.

Definition 1.5.3 (Not Missing At Random (NMAR)) The mechanism is called not missing at random if the distribution of $\delta$ depends on the missing values in the covariates. 
Note that the NMAR assumption is more natural in comparison with other mechanisms because one does not impose any assumptions on the missing probability mechanism.

\subsubsection{Methods for Analyzing Missing Data}

The literature on the analysis of partially missing data is comparatively recent. Afifi and Elashoff (1966) reviewed the literature on the problem of handling multivariate data with observations missing on some or all of the variables under study. They showed how the estimation problems can be simplified if the missing data follow certain patterns. Hartley and Hocking (1971) provided a simple taxonomy for incomplete-data problems and at the same time developed unified methods of analysis and finally in the well known paper by Dempster, Laird, and Rubin (1977), an applicable algorithm for computing maximum likelihood estimates was presented at various levels of generality. Methods proposed in this literature can, in general, be grouped into the following categories:

\section{Complete-Case Analysis}

Complete-case analysis is a common approach in which all incomplete observations are discarded and only the units with complete data are analyzed. This is a standard approach of most statistical software packages, e.g., Nei et al. (1975), used complete-case method in SPSS. This strategy is generally easy to carry out and may be satisfactory with small amounts of missing data. It is well-known that the complete-case method is inefficient, and may even give biased results if the missing data are not MCAR (see, Little and Rubin (2002)). 


\section{Weighted Complete Case Analysis}

This method is a modification of complete case analysis that differentially weights the complete cases to adjust for bias. The well-known Horvitz- Thompson estimator, (Horvitz and Thompson (1952)) for the population mean is a good example of this type of estimation that can be used for this method. This method works by weighting the complete cases by the inverse of the missing data probabilities. See, for example, Robins et al. (1994), Hirano et al. (2003), and Wang et al. (2004).

\section{Imputation-Based Procedures}

The missing values are filled-in (or imputed) and the resulting data are analyzed by standard methods. Commonly used procedures for imputation include hot deck imputation, mean imputation or regression imputation.

\section{Model-Based Procedures}

A broad class of procedures is generated by defining a model for the observed data and basing inferences on the likelihood or posterior under the model, with parameters estimated by procedures such as maximum likelihood. For more details, see, Little and Rubin (2002).

\subsection{Inequalities}

The following inequalities are main tools in proving the Theorems 1.3.4 and 1.3.5, and we will also use them many times throughout this research.

Theorem 1.6.1 (Hoeffding's Inequality; Hoeffding (1963)) Let $\mathbf{Z}_{1}, \cdots, \mathbf{Z}_{n}$ be independent bounded random variables such that $\mathbf{Z}_{i} \in\left[a_{i}, b_{i}\right]$ with probability 
one. Denote their sum by $S_{n}=\sum_{i=1}^{n} \mathbf{Z}_{i}$. Then for any $\epsilon>0$,

$$
P\left\{\left|S_{n}-E S_{n}\right|>\epsilon\right\} \leqslant 2 \exp \left(-\frac{2 \epsilon^{2}}{\sum_{i=1}^{n}\left(b_{i}-a_{i}\right)^{2}}\right) .
$$

Theorem 1.6.2 (Bernstein's Inequality; Bernstein (1946)) Let $\mathbf{Z}_{1}, \cdots, \mathbf{Z}_{n}$ be independent real-valued random variables with zero mean, and assume that $\mathbf{Z}_{i} \leqslant c$ with probability one. Let $\sigma^{2}=\frac{1}{n} \sum_{i=1}^{n} \operatorname{var}\left(\mathbf{Z}_{i}\right)$. Then, for any $\epsilon>0$,

$$
P\left\{\frac{1}{n} \sum_{i=n}^{n} \mathbf{Z}_{i}>\epsilon\right\} \leqslant \exp \left(-\frac{n \epsilon^{2}}{2 \sigma^{2}+2 c \epsilon / 3}\right) .
$$

Note that, Bernstein's inequality is better than Hoeffding's inequality when $\sigma \ll c$.

\subsection{Classification with Missing Data}

The traditional theory for estimation in classification gives no hint as to how to deal with missing values in the covariates. We mentioned some methods for handling missing values in Section 1.5. Chan and Dunn $(1972,1974)$ applied several commonly used methods of handling missing values in classification and studied the probabilities of correct classification by the Monte Carlo methods. Unfortunately, their study, in some aspect, is weak. The major drawback is that they imputed missing values based on mean substitution and the regression method but they did not pay attention to the point that by using these imputations, the misclassification errors increase. The following theorem clarifies that when information is missing, replacing $\mathbf{Z}$ by $t(\mathbf{Z})$ will increase the error:

Theorem 1.7.1 (Devroye et al. (1996), Chapter 3)

Let $t: \mathcal{R}^{d} \rightarrow \mathcal{R}^{p}$ be an arbitrary measurable function, then for any distribution of 
$(\mathbf{Z}, Y)$

$$
P\left\{\psi_{B}(\mathbf{Z}) \neq Y\right\} \leqslant P\left\{\psi_{B}^{*}\left(\mathbf{Z}^{*}\right) \neq Y\right\}
$$

where $\mathbf{Z}^{*}=t(\mathbf{Z})$, and $\psi_{B}$ and $\psi_{B}^{*}$ are the best classifiers in $\mathcal{R}^{d}$ and $\mathcal{R}^{p}$ respectively.

So, according to the above theorem, mean or regression imputation for missing values can increase the misclassification errors. Some other authors (e.g., Bahannon and Smith (1975)) construct the classification rule in the presence of missing data by estimating the parameters of the distributions. Anderson (1957) considered the maximum likelihood estimates of parameters of multivariate normal distributions with missing observations. Hocking and Smith (1968) developed a method for estimating the parameters in the multivariate normal distribution in which the missing observations are not restricted to follow certain patterns. These estimators are then substituted for the unknown parameters in the classification rule. In a recent paper, Chung and Hann (2000) focused on the classification of multivariate normals when a block of observations is missing. They proposed a new classification procedure which is a linear combination of two discriminant functions, one based on the complete samples and the other on the incomplete samples. The coefficients of this linear combination are chosen intuitively based on the sizes of complete and incomplete samples and Mahalanobis distances. This method has little or no merits and its usage has no theoretical justification. Note that all the work mentioned above, classify the new observation according to "Anderson's classification statistics" which is based on the strong assumption that two populations should be normal with identical covariance matrices, in addition, they completely ignored the missing probability mechanism. 


\subsection{Thesis Outline}

In Chapter 2, without imposing any assumptions on the missing probability mechanism, we derive representations for the best classifier corresponding to the setup where a block of covariates may be missing. An interesting and perhaps counter-intuitive feature of this classifier is that its misclassification error can sometimes be less than that of the optimal classifier corresponding to the case of no missing covariates. The contributions of Chapter 2 also include methods for constructing good classifiers without employing any imputation-based (or Horvitz-Thompson-type inverse weighting) arguments. The proposed nonparametric classifiers are good in the sense that they work without imposing any MAR-type assumptions, they do not depend on the distribution of the data, and they are strongly consistent. Both parametric and nonparametric situations are considered but the main focus is on the latter case.

In Chapter 3 we tackle the more realistic case where the missing values can appear anywhere in the vector of covariates, i.e., the so-called "Swiss-cheese" model. Once again, without imposing any assumptions on the missing probability mechanism, we derive a number of equivalent representations for the optimal classifier. These representations are then used to construct good classifiers based on popular local-averaging techniques ( such as kernels methods).

Chapter 4 uses the representations of the best classifier from Chapter 2 and 3 and contains two parts: In the first place, a histogram rule is proposed when some parts of covariates are missing. We consider both the case where there is only one missing pattern and the case that contains more than one missing pattern, referred to as 
"Swiss-cheese". In the second part of Chapter 4, data-based classifiers are derived using general partitioning rules. Once again, we consider the case where one block of covariates is missing as well as the full Swiss-cheese model. Strong consistency results for the proposed rules are also established.

In Chapter 5, least-squares approaches are proposed for classification in the presence of missing covariates. Both kernel-based imputation as well as HorvitzThompson-type inverse weighting approaches are employed to handle the presence of missing covariates. In the case of imputation, it is a certain regression function which is being imputed (and not the missing values). Using the theory of empirical processes, the performance of the resulting classifiers is assessed by obtaining exponential bounds on the deviations of their conditional errors from that of the Bayes classifier. Here the MAR assumption will be required.

Chapter 6 gives more numerical examples to illustrate the possible effects of missing covariates on the misclassification error rates. In addition, a simulation study is conducted to compare parametric and nonparametric classifiers for different missing probability mechanisms. For this part, the MCAR, MAR, and NMAR cases are considered, and the parametric classifiers with the kernel classifiers and the histogram rules are compared. Finally, to study the Swiss-cheese model, a real data set is considered. These data are classified nonparametrically based on the kernel rule and different situations are analyzed.

Chapter 7 discussed some future problems related to the material covered in the first five chapters. 


\section{Chapter 2}

\section{The Best Classifier in the Presence of Missing Covariates}

\subsection{Introduction}

Missing values in the covariates constitute a challenge in the daily work of statisticians. They often follow the approach of discarding the incomplete data and analyzing only the complete units. Since this method in most cases is biased especially when the complete cases are not a random sample of all the cases and it is also inefficient by increasing variance from the loss of information. Other methods such as imputation procedures have been proposed to try to rectify these problems. Commonly used procedures for imputation include hot deck imputation such that missing covariates are filled in and then the resulting completed data are analyzed by standard methods. In the recent works, some authors like Cheng and Chu (1996) and Hazelton (2000), have considered imputing a certain function of a missing value (and not the missing value itself). Also, Horvitz-Thompson inverse weighting meth- 
ods are employed to handle the presence of missing covariates. See, e.g., Robins et al. (1994), Hirano et al. (2003), and Wang et al. (2004); we will discuss these methods in Chapter 5. Note that all aforementioned (and many other) results in the literature are based on the MAR (Missing At Random) assumption imposed on the missing probability mechanism. Unlike the problems of point and curve estimation (where a curve can be a regression function, density function, or a distribution function), the area of classification in the presence of missing covariates has been barely touched. In this chapter, we show that the basic setup and previous results related to the best classifier are ill-suited when we are confronted with missing covariates. In fact, our main goal in this chapter is to find the best classifier when a block of covariates is missing. Section 2.2 is devoted to this task where it is shown that the proposed classifier is the best in the sense of minimizing the misclassification error; this is given by Theorem 2.2.1. We investigate the performance of our classifier numerically for different missing mechanisms in Example 1. Interestingly, the results in this example show that the misclassification error of the proposed classifier is sometimes less than that of the optimal classifier corresponding to the case of no missing covariates. The rest of this chapter deals with constructing sample versions of the best classifier. We consider both parametric and nonparametric situations and show that they are consistent. In the nonparametric case we focus on kernel classifiers. Both theory and the mechanics of the proposed procedures will be discussed.

\subsection{The Best Classifier}

In the case where one block of covariates is missing, our discussion and results are based on the following setup. Let $(\mathbf{Z}, Y) \sim F_{\mathbf{Z}, Y}$ be a random pair where 
$\mathbf{Z}=\left(\mathbf{X}^{\prime}, \mathbf{V}^{\prime}\right)^{\prime} \in \mathcal{R}^{p}$ is vector of covariates to be used to predict the class membership $Y \in\{0,1\}$. Now, consider the case where $\mathbf{X} \in \mathcal{R}^{d}, d \geqslant 1$, is always observable but $\mathbf{V} \in \mathcal{R}^{p-d}, p-d \geqslant 1$, could be missing. Also the missing data indicator is given by

$$
\delta= \begin{cases}1 & \text { if } \mathbf{V} \text { is not missing } \\ 0 & \text { otherwise }\end{cases}
$$

In this case, a random sample of size $n$ may be represented by

$$
\mathcal{D}_{n}:=\left\{\left(\mathbf{X}_{1}, \mathbf{V}_{1}, Y_{1}, \delta_{1}\right), \cdots,\left(\mathbf{X}_{n}, \mathbf{V}_{n}, Y_{n}, \delta_{n}\right)\right\}
$$

The missing pattern of covariates is shown in Figure 2.1 where $\star$ 's represent the missing covariates. Next, let $(\mathbf{X}, \mathbf{V}, Y, \delta) \stackrel{\text { i.i.d. }}{=}\left(\mathbf{X}_{1}, \mathbf{V}_{1}, Y_{1}, \delta_{1}\right)$ be a new/future observation for which $Y$ is to be predicted. Given $\mathcal{D}_{n}$ and $(\mathbf{Z}, \delta)$, how do we construct a Bayes consistent classifier? Unfortunately, this question cannot be answered unless one knows what the "Bayes" classifier is under the current setup, where $\mathbf{V}$ can be missing. Clearly, in the absence of any other information, if $\delta=1$ then the natural choice of a classifier is a map of the form $g_{1}: \mathcal{R}^{p} \rightarrow\{0,1\}$. On the other hand, when $\delta=0$ (i.e., $\mathbf{V}$ is missing), one is confined to classifiers of the form $g_{0}: \mathcal{R}^{d} \rightarrow\{0,1\}$. Thus, under the current setup, a classifier is any map of the form

$$
\begin{aligned}
\psi(\mathbf{Z}, \delta) \equiv \psi_{g_{1}, g_{0}}(\mathbf{Z}, \delta) & =I\{\delta=1\} g_{1}(\mathbf{Z})+I\{\delta=0\} g_{0}(\mathbf{X}) \\
& =\delta g_{1}(\mathbf{Z})+(1-\delta) g_{0}(\mathbf{X}) .
\end{aligned}
$$

We saw in Theorem 1.2.1 that the classifier defined by (1.1) was optimal when there were no missing covariates. So, one may suggest that an intuitively reasonable candidate for the best classifier, under the setup with missing covariates, is simply

$$
\breve{\psi}(\mathbf{Z}, \delta)=\delta \breve{\psi}_{1}(\mathbf{Z})+(1-\delta) \breve{\psi}_{0}(\mathbf{X})
$$


Figure 2.1: One block missing data pattern. Rows correspond to variables, columns to observations. $n=n_{1}+n_{2}$

$$
\begin{array}{ccccccc}
Z_{1,1} & Z_{1,2} & \cdots & Z_{1, n_{1}} & Z_{1, n_{1}+1} & \cdots & Z_{1, n_{1}+n_{2}} \\
Z_{2,1} & Z_{2,2} & \cdots & Z_{2, n_{1}} & Z_{2, n_{1}+1} & \cdots & Z_{2, n_{1}+n_{2}} \\
\vdots & \vdots & & \vdots & \vdots & & \vdots \\
Z_{d, 1} & Z_{d, 2} & \cdots & Z_{d, n_{1}} & Z_{d, n_{1}+1} & \cdots & Z_{d, n_{1}+n_{2}} \\
Z_{d+1,1} & Z_{d+1,2} & \cdots & Z_{d+1, n_{1}} & \star & \cdots & \star \\
\vdots & \vdots & & \vdots & \vdots & & \vdots \\
Z_{p, 1} & Z_{p, 2} & \cdots & Z_{p, n_{1}} & \star & \cdots & \star
\end{array}
$$

where

$$
\breve{\psi}_{1}(\mathbf{Z})=\left\{\begin{array}{ll}
1 & \text { if } E(Y \mid \mathbf{Z})>1 / 2 \\
0 & \text { otherwis, }
\end{array} \quad \breve{\psi}_{0}(\mathbf{X})= \begin{cases}1 & \text { if } E(Y \mid \mathbf{X})>1 / 2 \\
0 & \text { otherwise }\end{cases}\right.
$$

Although, there are some special cases where the classifier defined in (2.2) is the best classifier, it turns out, however, that this classifier is not optimal in general. In fact, the missing probability mechanism plays a crucial role in finding the best classifier. To appreciate this, consider the situation where a block of covariates is missing under the NMAR assumption. More specifically, the distribution of $\delta$ depends on the missing values (recall, $\mathbf{Z}^{\prime}=\left(\mathbf{X}^{\prime}, \mathbf{V}^{\prime}\right)$, where $\mathbf{V}$ may be missing). In order to find the best classifier, let

$$
p(\mathbf{Z}, Y) \equiv p(\mathbf{X}, \mathbf{V}, Y):=P(\delta=1 \mid \mathbf{X}, \mathbf{V}, Y)=E(\delta \mid \mathbf{X}, \mathbf{V}, Y)
$$

be the conditional probability that $\mathbf{V}$ is not missing (i.e., $\delta=1$ ), given $(\mathbf{Z}, Y)$. Also, 
let

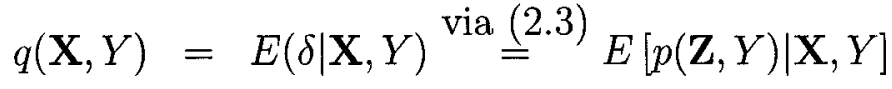

$$
\begin{aligned}
& \eta_{B}(\mathbf{Z})=E(Y \mid \mathbf{Z}) \\
& \lambda_{B}(\mathbf{X})=E(Y \mid \mathbf{X}) \\
& p_{1}(\mathbf{Z})=p(\mathbf{Z}, 1), p_{0}(\mathbf{Z})=p(\mathbf{Z}, 0) \\
& q_{1}(\mathbf{X})=q(\mathbf{X}, 1), q_{0}(\mathbf{X})=q(\mathbf{X}, 0) .
\end{aligned}
$$

Define the functions $\psi_{1}: \mathcal{R}^{p} \rightarrow\{0,1\}$ and $\psi_{0}: \mathcal{R}^{d} \rightarrow\{0,1\}$ by

$$
\begin{aligned}
& \psi_{1}(\mathbf{Z})= \begin{cases}1 & \text { if } p_{1}(\mathbf{Z}) \eta_{B}(\mathbf{Z})-p_{0}(\mathbf{Z})\left(1-\eta_{B}(\mathbf{Z})\right)>0 \\
0 & \text { otherwise }\end{cases} \\
& \psi_{0}(\mathbf{X})= \begin{cases}1 & \text { if }\left(1-q_{1}(\mathbf{X})\right) \lambda_{B}(\mathbf{X})-\left(1-q_{0}(\mathbf{X})\right)\left(1-\lambda_{B}(\mathbf{X})\right)>0 \\
0 & \text { otherwise }\end{cases}
\end{aligned}
$$

and put

$$
\psi_{B}(\mathbf{Z}, \delta)=\delta \psi_{1}(\mathbf{Z})+(1-\delta) \psi_{0}(\mathbf{X})
$$

Part (i) of the following theorem shows that $\psi_{B}$ is optimal in the sense of having the lowest misclassification error rate.

Theorem 2.2.1 Let $\psi_{B}$ be as in (2.11). Then for any other classifier $\psi$ given by (2.1), one has:

(i) $L(\psi)-L\left(\psi_{B}\right) \geqslant 0$, where $L(\psi)=P\{\psi(\mathbf{Z}, \delta) \neq Y\}$.

(ii) Suppose that the classifiers $g_{1}$ and $g_{0}$ in (2.1) are of the form $g_{1}(\mathbf{Z})=I\{G(\mathbf{Z})>$ $0\}$ and $g_{0}(\mathbf{X})=I\{H(\mathbf{X})>0\}$ for some $G: \mathcal{R}^{p} \rightarrow[-1,1]$ and $H: \mathcal{R}^{d} \rightarrow[-1,1]$. 
Then

$$
\begin{aligned}
L(\psi)-L\left(\psi_{B}\right) \leqslant & E\left|\left[p_{1}(\mathbf{Z}) \eta_{B}(\mathbf{Z})-p_{0}(\mathbf{Z})\left(1-\eta_{B}(\mathbf{Z})\right)\right]-G(\mathbf{Z})\right| \\
& +E\left|\left[\left(1-q_{1}(\mathbf{X})\right) \lambda_{B}(\mathbf{X})-\left(1-q_{0}(\mathbf{X})\right)\left(1-\lambda_{B}(\mathbf{X})\right)\right]-H(\mathbf{X})\right|
\end{aligned}
$$

\section{Proof.}

Part (i). Let $\psi$ be as in (2.1) and note that

$$
\begin{aligned}
P\{\psi(\mathbf{Z}, \delta)=Y\}= & P\left\{g_{1}(\mathbf{Z})=1, Y=1, \delta=1\right\}+P\left\{g_{1}(\mathbf{Z})=0, Y=0, \delta=1\right\} \\
& +P\left\{g_{0}(\mathbf{X})=1, Y=1, \delta=0\right\}+P\left\{g_{0}(\mathbf{X})=0, Y=0, \delta=0\right\} \\
:= & P_{1}+P_{2}+P_{3}+P_{4} .
\end{aligned}
$$

Upon conditioning on $(\mathbf{Z}, Y)$, one finds

$$
\begin{aligned}
P_{1}= & E\left[I\left\{g_{1}(\mathbf{Z})=1\right\} I\{Y=1\} P\{\delta=1 \mid \mathbf{Z}, Y\}\right]=E\left[I\left\{g_{1}(\mathbf{Z})=1\right\} Y p(\mathbf{Z}, Y)\right] \\
= & E\left[I\left\{g_{1}(\mathbf{Z})=1\right\} Y(Y p(\mathbf{Z}, 1)+(1-Y) p(\mathbf{Z}, 0))\right]=E\left[I\left\{g_{1}(\mathbf{Z})=1\right\} Y p_{1}(\mathbf{Z})\right] \\
& \quad \text { where } p_{1}(\mathbf{Z})=p(\mathbf{Z}, 1) \\
& =E\left[E\left[I\left\{g_{1}(\mathbf{Z})=1\right\} Y p_{1}(\mathbf{Z}) \mid \mathbf{Z}\right]\right]=E\left[I\left\{g_{1}(\mathbf{Z})=1\right\} \eta_{B}(\mathbf{Z}) p_{1}(\mathbf{Z})\right]
\end{aligned}
$$

Similarly, $P_{2}=E\left[I\left\{g_{1}(\mathbf{Z})=0\right\}\left(1-\eta_{B}(\mathbf{Z})\right) p_{0}(\mathbf{Z})\right], P_{3}=E\left[I\left\{g_{0}(\mathbf{X})=1\right\} \lambda_{B}(\mathbf{X})(1-\right.$ $\left.\left.q_{1}(\mathbf{X})\right)\right]$, and $P_{4}=E\left[I\left\{g_{0}(\mathbf{X})=0\right\}\left(1-\lambda_{B}(\mathbf{X})\right)\left(1-q_{0}(\mathbf{X})\right)\right]$, where $q_{y}(\mathbf{X})=$ $q(\mathbf{X}, y), y=0,1$. Therefore, 


$$
\begin{array}{rl} 
& L(\psi)-L\left(\psi_{B}\right) \\
=E & P\left\{\psi_{B}(\mathbf{Z}, \delta)=Y\right\}-P\{\psi(\mathbf{Z}, \delta)=Y\} \\
=E & E\left[p_{1}(\mathbf{Z}) \eta_{B}(\mathbf{Z})\left(I\left\{\psi_{1}(\mathbf{Z})=1\right\}-I\left\{g_{1}(\mathbf{Z})=1\right\}\right)\right. \\
& \left.+p_{0}(\mathbf{Z})\left(1-\eta_{B}(\mathbf{Z})\right)\left(I\left\{\psi_{1}(\mathbf{Z})=0\right\}-I\left\{g_{1}(\mathbf{Z})=0\right\}\right)\right] \\
& +E\left[\left(1-q_{1}(\mathbf{X})\right) \lambda_{B}(\mathbf{X})\left(I\left\{\psi_{0}(\mathbf{X})=1\right\}-I\left\{g_{0}(\mathbf{X})=1\right\}\right)\right. \\
& \left.+\left(1-q_{0}(\mathbf{X})\right)\left(1-\lambda_{B}(\mathbf{X})\right)\left(I\left\{\psi_{0}(\mathbf{X})=0\right\}-I\left\{g_{0}(\mathbf{X})=0\right\}\right)\right] \\
=E & {\left[\left(p_{1}(\mathbf{Z}) \eta_{B}(\mathbf{Z})-p_{0}(\mathbf{Z})\left(1-\eta_{B}(\mathbf{Z})\right)\right)\left(I\left\{\psi_{1}(\mathbf{Z})=1\right\}-I\left\{g_{1}(\mathbf{Z})=1\right\}\right)\right]} \\
& +E\left[\left(\left(1-q_{1}(\mathbf{X})\right) \lambda_{B}(\mathbf{X})-\left(1-q_{0}(\mathbf{X})\right)\left(1-\lambda_{B}(\mathbf{X})\right)\right)\right. \\
& \left.\quad \times\left(I\left\{\psi_{0}(\mathbf{X})=1\right\}-I\left\{g_{0}(\mathbf{X})=1\right\}\right)\right] \\
=E & {\left[\left|p_{1}(\mathbf{Z}) \eta_{B}(\mathbf{Z})-p_{0}(\mathbf{Z})\left(1-\eta_{B}(\mathbf{Z})\right)\right| I\left\{\psi_{1}(\mathbf{Z}) \neq g_{1}(\mathbf{Z})\right\}\right]} \\
& +E\left[\left|\left(1-q_{1}(\mathbf{X})\right) \lambda_{B}(\mathbf{X})-\left(1-q_{0}(\mathbf{X})\right)\left(1-\lambda_{B}(\mathbf{X})\right)\right| I\left\{\psi_{0}(\mathbf{X}) \neq g_{0}(\mathbf{X})\right\}\right] \\
\quad & \left.\quad \text { because of the definition of } \psi_{1} \text { and } \psi_{0}\right)
\end{array}
$$

$\geqslant 0$

part (ii). It was found in part (i) that $L(\psi)-L\left(\psi_{B}\right)=$ r.h.s. of (2.12). By considering the two cases $\left\{\psi_{1}(\mathbf{Z})=1, g_{1}(\mathbf{Z})=0\right\}$ and $\left\{\psi_{1}(\mathbf{Z})=0, g_{1}(\mathbf{Z})=1\right\}$ separately, (and recalling that $\left.g_{1}(\mathbf{Z})=I\{G(\mathbf{Z})>0\}\right)$, it is straightforward to see that the $1^{\text {st }}$ term on the r.h.s. of $(2.12)$ is bounded by $E\left|\left[p_{1}(\mathbf{Z}) \eta_{B}(\mathbf{Z})-p_{0}(\mathbf{Z})\left(1-\eta_{B}(\mathbf{Z})\right)\right]-G(\mathbf{Z})\right|$. Similarly, one finds that the $2^{\text {nd }}$ term on the r.h.s. of $(2.12)$ is bounded by $E\left|\left[\left(1-q_{1}(\mathbf{X})\right) \lambda_{B}(\mathbf{X})-\left(1-q_{0}(\mathbf{X})\right)\left(1-\lambda_{B}(\mathbf{X})\right)\right]-H(\mathbf{X})\right|$

Remark 2.2.2 Part (ii) of Theorem 2.2.1 gives upper bounds on the error difference 
$L(\psi)-L\left(\psi_{B}\right)$, where $\psi$ is any classifier of the form $\psi(\mathbf{Z}, \delta)=\delta I\{G(\mathbf{Z})>0\}+(1-$ $\delta) I\{H(\mathbf{X})>0\}$. Such bounds are particularly useful when the functions $G$ and $H$ are of the form $G(\mathbf{Z})=\widetilde{p}_{1}(\mathbf{Z}) \widetilde{\eta}_{1}(\mathbf{Z})-\widetilde{p}_{0}(\mathbf{Z}) \widetilde{\eta}_{0}(\mathbf{Z})$ and $H(\mathbf{X})=\left(1-\widetilde{q}_{1}(\mathbf{X})\right) \widetilde{\lambda}_{1}(\mathbf{X})-$ $\left(1-\widetilde{q}_{0}(\mathbf{X})\right) \widetilde{\lambda}_{0}(\mathbf{X})$. Here the functions $\widetilde{p}_{1}, \widetilde{p}_{0}, \widetilde{q}_{1}$, and $\widetilde{q}_{0}$ are approximations to $p_{1}, p_{0}, q_{1}$, and $q_{0}$, whereas $\widetilde{\eta}_{1}, \widetilde{\eta}_{0}, \widetilde{\lambda}_{1}$, and $\widetilde{\lambda}_{0}$ are approximations to $\eta_{B}, 1-\eta_{B}, \lambda_{B}$, and $1-\lambda_{B}$ respectively. If we denote the corresponding classifier by $\widetilde{\psi}(\mathbf{Z}, \delta)$ then, in view of part (ii) of Theorem 2.2.1, one can study the error difference $L(\widetilde{\psi})-L\left(\psi_{B}\right)$, where $L(\widetilde{\psi})=P\{\widetilde{\psi}(\mathbf{Z}, \delta) \neq Y\}$, by studying the $L_{1}$ differences $E\left|\widetilde{\eta}_{1}(\mathbf{Z})-\eta_{B}(\mathbf{Z})\right|$, $E\left|\widetilde{p}_{1}(\mathbf{Z})-p_{1}(\mathbf{Z})\right|$, etc.

It is worth noting that (2.3) does not impose any assumption on the missing probability mechanism. In the next remarks we will present the best classifiers under specific assumptions.

Remark 2.2.3 (The MAR Case) In the literature a common assumption on the missing mechanism, is the so-called MAR (Missing At Random) assumption that missingness depends only on the components that are observed. This corresponds to the simpler model where

$$
p(\mathbf{Z}, Y):=P\{\delta=1 \mid \mathbf{X}, \mathbf{V}, Y\}=P\{\delta=1 \mid \mathbf{X}, Y\} \stackrel{b y}{\stackrel{(2.4)}{=}} q(\mathbf{X}, Y),
$$

also $p_{1}(\mathbf{X})=p(\mathbf{X}, 1)$ and $p_{0}(\mathbf{X})=p(\mathbf{X}, 0)$.

\section{Remark 2.2.4 (Missingness Does Not Depend on Membership Class in}

the MAR Case) Consider the $M A R$ case where the probability that $\mathbf{V}$ is missing is the same for both classes. More precisely

$$
P\{\delta=1 \mid \mathbf{X}, \mathbf{V}, Y\}=P\{\delta=1 \mid \mathbf{X}\}:=p(\mathbf{X}) \equiv q(\mathbf{X})
$$


It is straightforward to see that in this case the naïve classifier defined in (2.2) is the best classifier.

Before going any further, we give a numerical example to illustrate the possible effects of missing mechanisms on misclassification error rates; 'NMAR', 'MAR' and 'MCAR' cases are considered.

\section{Example 1.}

This example deals with the prediction of the class membership $Y \in\{0,1\}$ of an entity based on the covariates $\mathbf{Z}=(X, V)^{\prime}$, where $\mathbf{Z} \sim N_{2}\left(\boldsymbol{\mu}_{0}, \Sigma_{0}\right)$ if $Y=0$ and $\mathbf{Z} \sim N_{2}\left(\boldsymbol{\mu}_{1}, \Sigma_{1}\right)$ otherwise. The parameters are chosen as follows

$$
\mu_{0}=\left(\begin{array}{l}
2 \\
2
\end{array}\right), \quad \mu_{1}=\left(\begin{array}{c}
0.5 \\
1
\end{array}\right), \quad \Sigma_{0}=\left(\begin{array}{cc}
1 & 0.15 \\
0.15 & 2.25
\end{array}\right), \quad \Sigma_{1}=\left(\begin{array}{cc}
1 & 0.15 \\
0.15 & 1
\end{array}\right)
$$

Here $X$ is observable but $V$ could be missing. The missing data mechanism is taken to be

$$
\begin{aligned}
p(\mathbf{z}, y)=p(x, v, y) & :=P\{\delta=1 \mid X=x, V=v, Y=y\} \\
& =e^{-a(1+d y)(x-1)^{2}-b(1+d y) v^{2}-d y-c}
\end{aligned}
$$

where $a, b, c, d \geqslant 0$ are constants. Note that, the reason for using the above model for the missing mechanism is its computational simplicity. Since the distribution of $\mathbf{Z}$ is normal, taking the exponential form for the missing mechanism would be extremely helpful. At the same time, the different values of $a, b, c$, and $d$ provide the different kinds of missing mechanisms (e.g., NMAR, MAR, etc). For example, the missingness of $\mathbf{Z}$ depends on the value of $\mathbf{V}$ if $b \neq 0$ (i.e., NMAR) and does not depend on $\mathbf{V}$ if $b=0$ (i.e., MAR). From the fact that $\eta_{B}(\mathbf{Z})=P\{Y=1 \mid \mathbf{Z}\}=$ $f_{1}(\mathbf{Z}) p /\left[f_{1}(\mathbf{Z}) p+f_{0}(\mathbf{Z})(1-p)\right]$ and $\lambda_{B}(X)=P\{Y=1 \mid X\}=g_{1}(X) p /\left[g_{1}(X) p+\right.$ 
$\left.g_{0}(X)(1-p)\right]$, it is straightforward to show that for the above bivariate normal populations $\psi_{1}(\mathbf{Z})$ and $\psi_{0}(X)$ in $(2.9)$ and (2.10) reduce to

$$
\begin{aligned}
& \psi_{1}(\mathbf{Z})= \begin{cases}1 & \text { if } p_{1}(\mathbf{Z}) f_{1}(\mathbf{Z}) p-p_{0}(\mathbf{Z}) f_{0}(\mathbf{Z})(1-p)>0 \\
0 & \text { otherwise }\end{cases} \\
& \psi_{0}(X)= \begin{cases}1 & \text { if }\left(1-q_{1}(X)\right) g_{1}(X) p-\left(1-q_{0}(X)\right) g_{0}(X)(1-p)>0 \\
0 & \text { otherwise },\end{cases}
\end{aligned}
$$

where

$$
\begin{aligned}
p_{1}(\mathbf{Z}) & =p(\mathbf{Z}, 1), \quad p_{0}(\mathbf{Z})=p(\mathbf{Z}, 0) \\
f_{k}(\mathbf{Z}) & =\text { density function for } N_{2}\left(\boldsymbol{\mu}_{k}, \Sigma_{k}\right), k=0,1 \\
g_{k}(X) & =\text { marginal density of } X \text { for class } k=0,1 \\
q_{1}(X) & =q(X, 1), \quad q_{0}(X)=q(X, 0),(q(X, Y) \text { is given by }(2.4)) \\
p & =P\{Y=1\}=1-P\{Y=0\} .
\end{aligned}
$$

Also put

$$
\psi_{B}(\mathbf{Z}, \delta)=\delta \psi_{1}(\mathbf{Z})+(1-\delta) \psi_{0}(X)
$$

In this numerical example, straightforward integration yields

$$
\begin{aligned}
& p_{1}(\mathbf{z})=\exp \left\{-a(1+d)(x-1)^{2}-b(1+d) v^{2}-d-c\right\} \\
& p_{0}(\mathbf{z})=\exp \left\{-a(x-1)^{2}-b v^{2}-c\right\}
\end{aligned}
$$

(by integration (in view of the far right side of (2.4)))

$$
\begin{gathered}
q_{1}(x)=(1+1.995 b(1+d))^{-\frac{1}{2}} \exp \left\{-c-d-a(1+d)(x-1)^{2}\right. \\
\left.-b(1+d)(1+0.15(x-0.5))^{2} /(1+1.995 b(1+d))\right\} \\
q_{0}(x)=(1+4.455 b)^{-\frac{1}{2}} \exp \left\{-a(x-1)^{2}-c-b(2+0.15(x-2))^{2} /(1+4.455 b)\right\} .
\end{gathered}
$$


As for the constants $a, b, c$, and $d$ in (2.13), the following choices were considered: $(a, b, c, d)=(0,0,0,0), \quad(0,0,1.5,0), \quad(1.5,0,1,0), \quad(0.3,0.2,1,2.5), \quad(0.3,0.2,0,2.5)$, $(0.4,0,0,0.5),(0.5,1.5,0.7,0.55),(0.15,0.2,0.1,3),(0,0,0,2.5)$. We have considered 3 values of $p=P\{Y=1\}: p=0.5, p=0.85$, and $p=0.25$. The results are shown in Tables 2.1, 2.2 and 2.3 respectively. These tables contain various misclassification probabilities for both (2.16) as well as the naïve classifier defined in (2.2), where

$$
\begin{aligned}
& \breve{\psi}_{1}(\mathbf{Z})= \begin{cases}1 & \text { if } f_{1}(\mathbf{Z}) p-f_{0}(\mathbf{Z})(1-p)>0 \\
0 & \text { otherwise }\end{cases} \\
& \breve{\psi}_{0}(X)= \begin{cases}1 & \text { if } g_{1}(X) p-g_{0}(X)(1-p)>0 \\
0 & \text { otherwise. }\end{cases}
\end{aligned}
$$

Table 2.1: Error rates for $\psi_{B}$ and $\psi_{\text {naïve }}$ when $p=0.5$

\begin{tabular}{|c|c|c|c|c|c|c|}
\hline$a$ & $b$ & $c$ & $d$ & Error $\left(\psi_{B}\right)$ & Error $\left(\psi_{\text {naïe }}\right)$ & Missing Mechanism \\
\hline 0 & 0 & 0 & 0 & 0.198 & 0.198 & No missing data \\
0 & 0 & 1.5 & 0 & 0.220 & 0.220 & MCAR \\
1.5 & 0 & 1 & 0 & 0.219 & 0.219 & MAR \\
0.3 & 0.2 & 1 & 2.5 & 0.209 & 0.232 & NMAR \\
0.3 & 0.2 & 0 & 2.5 & 0.169 & 0.241 & NMAR \\
0.4 & 0 & 0 & 0.5 & 0.153 & 0.206 & MAR \\
0.5 & 1.5 & 0.7 & 0.55 & 0.225 & 0.227 & NMAR \\
0.15 & 0.2 & 0.1 & 3 & 0.170 & 0.239 & NMAR \\
0 & 0 & 0 & 2.5 & 0.036 & 0.215 & MAR \\
\hline
\end{tabular}


Table 2.2: Error rates for $\psi_{B}$ and $\psi_{\text {naïve }}$ when $p=0.85$

\begin{tabular}{|c|c|c|c|c|c|c|}
\hline$a$ & $b$ & $c$ & $d$ & Error $\left(\psi_{B}\right)$ & Error $\left(\psi_{\text {naïe }}\right)$ & Missing Mechanism \\
\hline 0 & 0 & 0 & 0 & 0.106 & 0.106 & No missing data \\
0 & 0 & 1.5 & 0 & 0.119 & 0.119 & MCAR \\
1.5 & 0 & 1 & 0 & 0.121 & 0.121 & MAR \\
0.3 & 0.2 & 1 & 2.5 & 0.113 & 0.124 & NMAR \\
0.3 & 0.2 & 0 & 2.5 & 0.095 & 0.124 & NMAR \\
0.4 & 0 & 0 & 0.5 & 0.106 & 0.111 & MAR \\
0.5 & 1.5 & 0.7 & 0.55 & 0.122 & 0.123 & NMAR \\
0.15 & 0.2 & 0.1 & 3 & 0.090 & 0.124 & NMAR \\
0 & 0 & 0 & 2.5 & 0.039 & 0.107 & MAR \\
\hline
\end{tabular}

Here, the entries in Tables 2.1, 2.2 and 2.3 are the exact misclassification probabilities which are computable (via integration) because the full knowledge of the two class distributions as well as the form of the missing probability mechanism, $p(x, v, y)$, is completely given. (The proof of Theorem 2.2.1 shows what integrations one has to carry out to compute the exact misclassification error rate.) Observe that the error rate of the classifier $\psi_{B}$ is always less than or equal to that of the naïve classifier, $\psi_{\text {naive }}$. (In fact, according to Theorem 2.2.1, $\psi_{B}$ has the lowest error rate.) The case where $a=b=c=d=0$ corresponds to $P\{\delta=1 \mid \mathbf{Z}, Y\}=1$, i.e., there are no missing covariates; in this case the error rates are $0.198,0.106$ and 0.159 corresponding to $p=0.5, p=0.85$ and $p=0.25$, respectively. The misclassification errors for $\psi_{B}$ and $\psi_{\text {naive }}$ are equal, as expected. Also note that the misclassification errors in the 
Table 2.3: Error rates for $\psi_{B}$ and $\psi_{\text {naïve }}$ when $p=0.25$

\begin{tabular}{|c|c|c|c|c|c|c|}
\hline$a$ & $b$ & $c$ & $d$ & Error $\left(\psi_{B}\right)$ & Error $\left(\psi_{\text {naïve }}\right)$ & Missing Mechanism \\
\hline 0 & 0 & 0 & 0 & 0.159 & 0.159 & No missing data \\
0 & 0 & 1.5 & 0 & 0.171 & 0.171 & MCAR \\
1.5 & 0 & 1 & 0 & 0.170 & 0.170 & MAR \\
0.3 & 0.2 & 1 & 2.5 & 0.166 & 0.179 & NMAR \\
0.3 & 0.2 & 0 & 2.5 & 0.145 & 0.187 & NMAR \\
0.4 & 0 & 0 & 0.5 & 0.133 & 0.168 & MAR \\
0.5 & 1.5 & 0.7 & 0.55 & 0.175 & 0.177 & NMAR \\
0.15 & 0.2 & 0.1 & 3 & 0.145 & 0.177 & NMAR \\
0 & 0 & 0 & 2.5 & 0.019 & 0.176 & MAR \\
\hline
\end{tabular}

second row for both classifiers are equal. This is because $a=b=d=0$ and $c=1.5$, correspond to the MCAR case; see Remark 2.2.4. The results on the third row in the tables show that both classifiers behave the same even in the MAR case. Remark 2.2.4, shows that this is indeed to be expected. Interestingly, the last rows also correspond to the MAR case, but there is a huge difference between the error rates of the two classifiers. Also in this case, there is a significant difference between the misclassification error of $\psi_{B}$ and that of the optimal classifier corresponding to the case of no missing covariates (for example, in Table 2.1 the error of $\psi_{B}$ in the last row is 0.036 and in the first row, when there are no missing data, is 0.198). This is related to the crucial role of $Y$ in missing probability mechanisms; in the last rows of the Tables 2.1, 2.2, and 2.3, the missing probabilities depend on $Y$ only. The results in these tables also show some other cases where the misclassification error of $\psi_{B}$ can be less than the misclassification error of the optimal classifier for the case in 
which there is no missing data. See, for example, the row corresponding to $a=0.15$, $b=0.20, c=0.1$ and $d=3.0$, in these tables. In other words, classification with missing covariates can sometimes have a lower misclassification error rate than the case with no missing covariates. An intuitive reason is that, although $\mathbf{V}$ is missing, but the random variable $\delta$ (which is always observable) may have more information about $Y$ than $\mathbf{V}$ has. This, in turn, can result in lower error rates when predicting $Y$. Meanwhile, in all three tables the misclassification errors of $\psi_{\text {naive }}$ are increased in comparison with the errors reported in the first rows of these tables.

\subsection{Sample Versions of the Best Classifier}

As Theorem 2.2.1 shows the classifier defined in (2.11) is the best classifier in general. In practice, however, either the underlying distributions or the missing probability mechanism (or both), are always unknown and one only has access to some data (i.i.d.), that is to

$$
\mathcal{D}_{n}:=\left\{\left(\mathbf{X}_{1}, \mathbf{V}_{1}, Y_{1}, \delta_{1}\right), \cdots,\left(\mathbf{X}_{n}, \mathbf{V}_{n}, Y_{n}, \delta_{n}\right)\right\}
$$

Therefore, we have to construct a classifier based on the sample $\mathcal{D}_{n}$. The simplest and perhaps the most natural sample version of $\psi_{B}$ in $(2.11)$ where $\psi_{1}$ and $\psi_{0}$ defined in (2.9) and (2.10) is the plug-in version in which the quantities $p_{i}(\cdot), q_{i}(\cdot), \eta_{B}(\cdot), \lambda_{B}(\cdot), i=0,1$, are replaced by some data-based counterparts $\widehat{p}_{i}(\cdot), \widehat{q}_{i}(\cdot), \widehat{\eta}(\cdot)$, and $\widehat{\lambda}(\cdot)$. In order to study the performance of the resulting classifiers, we first state the following general result. Let $G: \mathcal{R}^{p} \rightarrow[-1,1]$ and $H: \mathcal{R}^{d} \rightarrow[-1,1]$ be as in part (ii) of Theorem 2.2.1. Also, let $G_{n}$ and $H_{n}$ be any sample versions of the functions $G$ and $H$, and put $g_{n 1}(\mathbf{Z})=I\left\{G_{n}(\mathbf{Z})>0\right\}$ and 
$g_{n 0}(\mathbf{X})=I\left\{H_{n}(\mathbf{X})>0\right\}$. Define the classifier

$$
\psi_{n}(\mathbf{Z}, \delta)=\delta g_{n 1}(\mathbf{Z})+(1-\delta) g_{n 0}(\mathbf{X})
$$

The following corollary may be viewed as the counterpart of part (ii) of Theorem 2.2.1 for the classifier $\psi_{n}$.

Corollary 2.3.1 Let $\psi_{n}$ be as above. Then

$$
\begin{aligned}
L_{n}\left(\psi_{n}\right)-L\left(\psi_{B}\right) \leqslant E & {\left[\left|\left[p_{1}(\mathbf{Z}) \eta_{B}(\mathbf{Z})-p_{0}(\mathbf{Z})\left(1-\eta_{B}(\mathbf{Z})\right)\right]-G_{n}(\mathbf{Z})\right| \mid \mathcal{D}_{n}\right] } \\
+ & E\left[\mid\left[\left(1-q_{1}(\mathbf{X})\right) \lambda_{B}(\mathbf{X})-\left(1-q_{0}(\mathbf{X})\right)\left(1-\lambda_{B}(\mathbf{X})\right)\right]\right. \\
& -H_{n}(\mathbf{X})||_{\left.\mathcal{D}_{n}\right]}
\end{aligned}
$$

where $L_{n}\left(\psi_{n}\right)=P\left\{\psi_{n}(\mathbf{Z}, \delta) \neq Y \mid \mathcal{D}_{n}\right\}$.

The upper bounds in the above corollary are particulary useful when the functions $G_{n}$ and $H_{n}$ are of the following forms

$$
\begin{aligned}
& G_{n}(\mathbf{Z})=\widehat{p}_{1}(\mathbf{Z}) \widehat{\eta}(\mathbf{Z})-\widehat{p}_{0}(\mathbf{Z})(1-\widehat{\eta}(\mathbf{Z})) \\
& H_{n}(\mathbf{X})=\left(1-\widehat{q}_{1}(\mathbf{X})\right) \widehat{\lambda}(\mathbf{X})-\left(1-\widehat{q}_{0}(\mathbf{X})\right)(1-\widehat{\lambda}(\mathbf{X}))
\end{aligned}
$$

where $\widehat{p}_{1}(\cdot), \widehat{p}_{0}(\cdot), \widehat{q}_{1}(\cdot), \widehat{q}_{0}(\cdot), \widehat{\eta}(\cdot)$, and $\widehat{\lambda}(\cdot)$ be estimates of $p_{1}(\cdot), p_{0}(\cdot), q_{1}(\cdot), q_{0}(\cdot)$, $\eta_{B}(\cdot)$, and $\lambda_{B}(\cdot)$. Then Corollary 2.3 .1 yields

$$
\begin{aligned}
L_{n}\left(\psi_{n}\right)-L\left(\psi_{B}\right) \leqslant & E\left[\left|\widehat{p}_{1}(\mathbf{Z}) \widehat{\eta}(\mathbf{Z})-p_{1}(\mathbf{Z}) \eta_{B}(\mathbf{Z})\right| \mid \mathcal{D}_{n}\right] \\
& +E\left[\left|\widehat{p}_{0}(\mathbf{Z})(1-\widehat{\eta}(\mathbf{Z}))-p_{0}(\mathbf{Z})\left(1-\eta_{B}(\mathbf{Z})\right)\right| \mid \mathcal{D}_{n}\right] \\
& +E\left[\left|\left(1-\widehat{q}_{1}(\mathbf{X})\right) \widehat{\lambda}(\mathbf{X})-\left(1-q_{1}(\mathbf{X})\right) \lambda_{B}(\mathbf{X})\right| \mid \mathcal{D}_{n}\right] \\
& +E\left[\left|\left(1-\widehat{q}_{0}(\mathbf{X})\right)(1-\widehat{\lambda}(\mathbf{X}))-\left(1-q_{0}(\mathbf{X})\right)\left(1-\lambda_{B}(\mathbf{X})\right)\right| \mid \mathcal{D}_{n}\right]
\end{aligned}
$$


Thus one only needs to study,

$$
\begin{aligned}
& E\left[\left|\widehat{p}_{1}(\mathbf{Z})-p_{1}(\mathbf{Z})\right| \mid \mathcal{D}_{n}\right], \quad E\left[\left|\widehat{p}_{0}(\mathbf{Z})-p_{0}(\mathbf{Z})\right| \mid \mathcal{D}_{n}\right] \\
& E\left[\left|\widehat{q}_{1}(\mathbf{X})-q_{1}(\mathbf{X})\right| \mid \mathcal{D}_{n}\right], \quad E\left[\left|\widehat{q}_{0}(\mathbf{X})-q_{0}(\mathbf{X})\right| \mid \mathcal{D}_{n}\right] \\
& E\left[\left|\widehat{\eta}(\mathbf{Z})-\eta_{B}(\mathbf{Z})\right| \mid \mathcal{D}_{n}\right], \text { and } E\left[\left|\widehat{\lambda}(\mathbf{X})-\lambda_{B}(\mathbf{X})\right| \mid \mathcal{D}_{n}\right]
\end{aligned}
$$

In the next sections, we consider parametric and nonparametric estimators and investigate the Bayes consistency of resulting classifiers. Interestingly, we describe in the next section that it is not possible to have parametric estimation for $\widehat{p}_{1}(\cdot), \widehat{p}_{0}(\cdot), \widehat{q}_{1}(\cdot)$, and $\widehat{q}_{0}(\cdot)$, since the missing mechanism is always unknown.

\subsection{Parametric Estimation}

In parametric cases, where the distribution of $\mathbf{Z}$ is known up to some unknown parameters, one may define, (see, Section 1.3.1)

$$
\eta_{\boldsymbol{\theta}}(\mathbf{Z})=P\{Y=1 \mid \mathbf{Z}\}=\frac{f_{1}\left(\mathbf{Z}, \boldsymbol{\theta}_{1}\right) p}{f_{1}\left(\mathbf{Z}, \boldsymbol{\theta}_{1}\right) p+f_{0}\left(\mathbf{Z}, \boldsymbol{\theta}_{0}\right)(1-p)},
$$

and

$$
\lambda_{\boldsymbol{\theta}}(\mathbf{X})=P\{Y=1 \mid \mathbf{X}\}=\frac{g_{1}\left(\mathbf{X}, \boldsymbol{\theta}_{1}\right) p}{g_{1}\left(\mathbf{X}, \boldsymbol{\theta}_{1}\right) p+g_{0}\left(\mathbf{X}, \boldsymbol{\theta}_{0}\right)(1-p)}
$$

where

$$
\begin{aligned}
f_{k}\left(\mathbf{Z}, \boldsymbol{\theta}_{k}\right) & =\text { density function for } \mathbf{Z} \text { for class } k=0,1 \\
g_{k}\left(\mathbf{X}, \boldsymbol{\theta}_{k}\right) & =\text { marginal density of } \mathbf{X} \text { for class } k=0,1 \\
\boldsymbol{\theta} & =\left(\boldsymbol{\theta}_{0}, \boldsymbol{\theta}_{1}, p\right)=\text { the parameter vector } \\
p & =P\{Y=1\}=1-P\{Y=0\} .
\end{aligned}
$$


Then, the parametric forms of (2.9) and (2.10) are

$$
\begin{aligned}
& \psi_{1 \boldsymbol{\theta}}(\mathbf{Z})= \begin{cases}1 & \text { if } p_{1}(\mathbf{Z}) \eta_{\boldsymbol{\theta}}(\mathbf{Z})-p_{0}(\mathbf{Z})\left(1-\eta_{\boldsymbol{\theta}}(\mathbf{Z})\right)>0 \\
0 & \text { otherwise }\end{cases} \\
& \psi_{0 \boldsymbol{\theta}}(\mathbf{X})= \begin{cases}1 & \text { if }\left(1-q_{1}(\mathbf{X})\right) \lambda_{\boldsymbol{\theta}}(\mathbf{X})-\left(1-q_{0}(\mathbf{X})\right)\left(1-\lambda_{\boldsymbol{\theta}}(\mathbf{X})\right)>0 \\
0 & \text { otherwise. }\end{cases}
\end{aligned}
$$

Finally, the Bayes rule can be rewritten as

$$
\psi_{B}(\mathbf{Z}, \delta)=\delta \psi_{1 \boldsymbol{\theta}}(\mathbf{Z})+(1-\delta) \psi_{0 \boldsymbol{\theta}}(\mathbf{X})
$$

To find the sample version of the above classifier, we construct $\widehat{\boldsymbol{\theta}}_{1}, \widehat{\boldsymbol{\theta}}_{0}, \widehat{p}$ and also $\widehat{p}_{i}$, and $\widehat{q}_{i}, i=0,1$ from the data, and use them to form classifiers

$$
\begin{aligned}
& \psi_{\mathbf{1} \hat{\boldsymbol{\theta}}}(\mathbf{Z})= \begin{cases}1 & \text { if } \widehat{p}_{1}(\mathbf{Z}) \eta_{\hat{\boldsymbol{\theta}}}(\mathbf{Z})-\widehat{p}_{0}(\mathbf{Z})\left(1-\eta_{\hat{\boldsymbol{\theta}}}(\mathbf{Z})\right)>0 \\
0 & \text { otherwise }\end{cases} \\
& \psi_{0 \hat{\boldsymbol{\theta}}}(\mathbf{X})= \begin{cases}1 & \text { if }\left(1-\widehat{q}_{1}(\mathbf{X})\right) \lambda_{\hat{\boldsymbol{\theta}}}(\mathbf{X})-\left(1-\widehat{q}_{0}(\mathbf{X})\right) \lambda_{\hat{\boldsymbol{\theta}}}(\mathbf{X})>0 \\
0 & \text { otherwise },\end{cases}
\end{aligned}
$$

also, put

$$
\psi_{\hat{\boldsymbol{\theta}}}(\mathbf{Z}, \delta)=\delta \psi_{1 \hat{\boldsymbol{\theta}}}(\mathbf{Z})+(1-\delta) \psi_{0 \hat{\boldsymbol{\theta}}}(\mathbf{X})
$$

The following theorem which is based on Devroye et al. (1996) investigates the consistency of classifier defined in (2.20). (See Theorem 1.3.1.)

Theorem 2.4.1 If $\eta_{\boldsymbol{\theta}}$ and $\lambda_{\boldsymbol{\theta}}$ are continuous in $\boldsymbol{\theta}$ w.r.t. the $L_{1}$ metric, and $\widehat{\boldsymbol{\theta}} \stackrel{P}{\rightarrow} \boldsymbol{\theta}$, then $\psi_{\hat{\boldsymbol{\theta}}}$ in (2.20) is Bayes consistent in the $L_{1}$ sense (i.e., $\left.E\left[L_{n}\left(\psi_{\hat{\boldsymbol{\theta}}}\right)\right] \rightarrow L\left(\psi_{B}\right)\right)$, provided that $E\left[\left|\widehat{p}_{i}(\mathbf{Z})-p_{i}(\mathbf{Z})\right| \mid \mathcal{D}_{n}\right] \stackrel{P}{\rightarrow} 0$ and $E\left[\left|\widehat{q}_{i}(\mathbf{X})-q_{i}(\mathbf{X})\right| \mid \mathcal{D}_{n}\right] \stackrel{P}{\rightarrow} 0, i=0,1$. 


\section{Proof.}

By using Corollary 2.3.1

$$
\begin{aligned}
L_{n}\left(\psi_{\hat{\boldsymbol{\theta}}}\right)-L\left(\psi_{B}\right) & \leqslant \sum_{i=0,1} E\left[\left|\widehat{p}_{i}(\mathbf{Z})-p_{i}(\mathbf{Z})\right| \mid \mathcal{D}_{n}\right]+\sum_{i=0,1} E\left[\left|\widehat{q}_{i}(\mathbf{X})-q_{i}(\mathbf{X})\right| \mid \mathcal{D}_{n}\right] \\
& +2 E\left[\left|\eta_{\hat{\boldsymbol{\theta}}}(\mathbf{Z})-\eta_{\boldsymbol{\theta}}(\mathbf{Z})\right| \mid \mathcal{D}_{n}\right]+2 E\left[\left|\lambda_{\hat{\boldsymbol{\theta}}}(\mathbf{X})-\lambda_{\boldsymbol{\theta}}(\mathbf{X})\right| \mid \mathcal{D}_{n}\right] .
\end{aligned}
$$

The proof now follows from the statement of the theorem together with the Lebesgue dominated convergence theorem.

The rest of this section is devoted to proposing suitable methods for estimating $\boldsymbol{\theta}, p_{i}$, and $q_{i}, i=0,1$.

\subsubsection{Parameters Estimation}

In this section we briefly discuss two methods for estimating the parameters of the underlying distributions.

The Expectation Maximization (EM) Algorithm.

The EM algorithm (Dempster, Laird and Rubin (1977)) is a general iterative technique for finding maximum likelihood estimates for parametric models when the data are incomplete. Each iteration of EM consists of an M step (maximization step), which estimates the parameters by maximum likelihood estimation just as if there were no missing data, and an E step (expectation step), which finds the conditional expectation of "missing data" given the observed data and current parameter estimates. It then substitutes these values for the "missing data". There are some extensions of EM algorithm like ECM (expectation conditional maximization), ECME (expectation conditional maximization either), etc. These are discussed in Little and Rubin (2002) and Schafer (1997). 


\section{Anderson's Method.}

Anderson (1957) introduced factored likelihoods for the important case of the multivariate normal. He used the maximum likelihood method to estimate means, variances and correlation of $\mathbf{X}$ and $\mathbf{V}$ (recall, $\mathbf{Z}^{\prime}=\left(\mathbf{X}^{\prime}, \mathbf{V}^{\prime}\right)$ ) by assuming that $\mathbf{Z}$ has normal distribution with the following parameters

$$
\boldsymbol{\mu}_{z}=\left(\begin{array}{l}
\boldsymbol{\mu}_{x} \\
\boldsymbol{\mu}_{v}
\end{array}\right), \quad \Sigma_{z z}=\left(\begin{array}{cc}
\Sigma_{x x} & \Sigma_{x v} \\
\Sigma_{x v} & \Sigma_{v v}
\end{array}\right)
$$

This method, factors the joint distributions of $\mathbf{X}$ and $\mathbf{V}$ into the marginal distributions of $\mathbf{X}$ and the conditional distribution of $\mathbf{V}$ given $\mathbf{X}$. The ML estimates of parameters are

$$
\begin{gathered}
\widehat{\boldsymbol{\mu}}_{x}=\overline{\mathbf{X}}=\frac{1}{n} \sum_{i=1}^{n} \mathbf{X}_{i}, \quad \widehat{\boldsymbol{\mu}}_{v}=\bar{V}^{*}+\widehat{B}\left(\overline{\mathbf{X}}-\overline{\mathbf{X}}^{*}\right) \\
\widehat{\Sigma}_{x x}=\frac{1}{n} \sum_{i=1}^{n}\left(\mathbf{X}_{i}-\overline{\mathbf{X}}\right)\left(\mathbf{X}_{i}-\overline{\mathbf{X}}\right)^{\prime}, \quad \widehat{\Sigma}_{x v}=\widehat{B} \widehat{\Sigma}_{x x}, \quad \widehat{\Sigma}_{v v}=\widehat{\Sigma}_{v \cdot x}+\widehat{B} \widehat{\Sigma}_{x x} \widehat{B}^{\prime},
\end{gathered}
$$

where

$$
\begin{gathered}
\overline{\mathbf{X}}^{*}=\frac{1}{n_{1}} \sum_{i=1}^{n_{1}} \mathbf{X}_{i} \quad \overline{\mathbf{V}}^{*}=\frac{1}{n_{1}} \sum_{i=1}^{n_{1}} \mathbf{V}_{i} \\
\widehat{B}=\sum_{i=1}^{n_{1}}\left(\mathbf{V}_{i}-\overline{\mathbf{V}}^{*}\right)\left(\mathbf{X}_{i}-\overline{\mathbf{X}}^{*}\right)^{\prime}\left[\sum_{i=1}^{n_{1}}\left(\mathbf{X}_{i}-\overline{\mathbf{X}}^{*}\right)\left(\mathbf{X}_{i}-\overline{\mathbf{X}}^{*}\right)^{\prime}\right]^{-1} \\
\widehat{\Sigma}_{v \cdot x}=\frac{1}{n_{1}}\left[\sum_{i=1}^{n_{1}}\left(\mathbf{V}_{i}-\overline{\mathbf{V}}^{*}\right)\left(\mathbf{V}_{i}-\overline{\mathbf{V}}^{*}\right)^{\prime}-\widehat{B} \sum_{i=1}^{n_{1}}\left(\mathbf{X}_{i}-\overline{\mathbf{X}}^{*}\right)\left(\mathbf{X}_{i}-\overline{\mathbf{X}}^{*}\right)^{\prime} \widehat{B}^{\prime}\right]
\end{gathered}
$$

Note that, $n_{1}$ is the number of data that are completely observed.

\subsubsection{Missing Probabilities Estimation}

To estimate $p_{i}, q_{i}, i=0,1$, we shall make the realistic assumption that the functional form of the missing probability mechanism, $p_{i}(\mathbf{Z})=P\{\delta=1 \mid \mathbf{Z}, Y=i\}$, and $q_{i}(\mathbf{X})=$ 
$P\{\delta=1 \mid \mathbf{X}, Y=i\}, i=0,1$ is completely unknown and hence $\widehat{p}_{i}$ and $\widehat{q}_{i}, i=0,1$ will be nonparametric estimates. Clearly the nonparametric estimation of $p_{0}$ and $p_{1}$ can be problematic unless one can impose further assumptions on the missing probability mechanism. To see this, consider any local averaging estimator for $p(\mathbf{z}, y)$ :

$$
\widehat{p}(\mathbf{z}, y)=\frac{\sum_{i=1}^{n} \delta_{i} \omega_{n i}(\mathbf{z}, y)}{\sum_{i=1}^{n} \omega_{n i}(\mathbf{z}, y)} \quad y=0,1 \text {. }
$$

where $\omega_{n i}(\mathbf{z}, y) \equiv \omega_{n}\left(\mathbf{z}, y, \mathbf{Z}_{i}, Y_{i}\right)$ are weight functions. The class of estimators (2.21) contains virtually all popular nonparametric estimators; this includes kernel estimators, nearest neighbor estimators, histogram, as well as general partitioning estimators; see Section 1.3.3 for more details. Unfortunately, these popular estimators are not readily computable, because

$$
\sum_{i=1}^{n} \omega_{n i}(\mathbf{z}, y)=\sum_{i=1}^{n} \delta_{i} \omega_{n i}(\mathbf{z}, y)+\sum_{i=1}^{n}\left(1-\delta_{i}\right) \omega_{n i}(\mathbf{z}, y)
$$

and the second term above is not computable since $\omega_{n i}(\mathbf{z}, y) \equiv \omega_{n i}\left(\mathbf{z}, y, \mathbf{Z}_{i}, Y_{i}\right)$ is not available when $\delta_{i}=0$. However, any popular nonparametric estimator can be used to estimate $p_{i}$ and $q_{i}, i=0,1$ under the additional assumption that the missing mechanism is MAR, i.e.,

$$
p(\mathbf{Z}, Y):=P\{\delta=1 \mid \mathbf{X}, \mathbf{V}, Y\} \stackrel{\text { MAR }}{=} P\{\delta=1 \mid \mathbf{X}, Y\}:=q(\mathbf{X}, Y) .
$$

The above MAR assumption implies that $p_{i}(\mathbf{Z})=q_{i}(\mathbf{X}), i=0,1$, and the estimator for $q_{i}(\mathbf{x})=q(\mathbf{x}, i)$ is

$$
\widehat{q}(\mathbf{x}, y)=\frac{\sum_{i=1}^{n} \delta_{i} \omega_{n i}^{*}(\mathbf{x}, y)}{\sum_{i=1}^{n} \omega_{n i}^{*}(\mathbf{x}, y)} \quad y=0,1
$$

where $\omega_{n i}^{*}(\mathbf{x}, y) \equiv \omega_{n}^{*}\left(\mathbf{x}, y, \mathbf{X}_{i}, Y_{i}\right)$ is available. Under the conditions of Stone's theorem (see, Theorem 1.3.2) $E\left[\left|\widehat{q}_{i}(\mathbf{X})-q_{i}(\mathbf{X})\right| \mathcal{D}_{n}\right] \stackrel{P}{\rightarrow} 0, i=0,1$. In Chapter 5, we propose kernel-type estimation for $q_{1}$ and $q_{0}$ and show the stronger result that $E\left[\left|\widehat{q}_{i}(\mathbf{X})-q_{i}(\mathbf{X})\right| \mid \mathcal{D}_{n}\right] \stackrel{\text { a.s }}{\rightarrow} 0$. 


\subsection{Nonparametric Estimation}

We saw in the previous section that to apply parametric methods, one needed to know the distribution of $\mathbf{Z}$ and, also, to impose the MAR assumption. When there is no information available about the form of $f_{i}, g_{i}$ or the form of $\eta_{B}(\cdot)$, (and $\lambda_{B}$ ) or the missing probability mechanism $p(\mathbf{X}, \mathbf{V}, Y)$, most of the estimation has to be carried out in a nonparametric fashion. Here, the nonparametric estimation of $\eta_{B}(\mathbf{Z})=E(Y \mid \mathbf{Z})$ can be particularly difficult due to the presence of missing covariates. Estimators based on the complete cases alone are typically not unbiased (not even asymptotically). Alternatively, one may use imputation-based methods to take into account the incomplete cases, or employ a Horvitz-Thompson-type inverse weighting approach to handle the missing covariates. The theoretical validity of the resulting classifiers (at least, in the sense of consistency), requires further restrictive assumptions on the missing probability mechanism as well as the underlying distribution of the data. To avoid such problems and, more importantly, to avoid further parametric/distributional assumptions, or MAR-type assumptions, (which may not be warranted), we start by proposing a kernel-based classifier as follows. First note that the classifier $\psi_{B}$ in $(2.11)$, i.e.,

$$
\psi_{B}(\mathbf{Z}, \delta)=\delta \psi_{1}(\mathbf{Z})+(1-\delta) \psi_{0}(\mathbf{X})
$$

where $\psi_{1}$ and $\psi_{0}$ are as in $(2.9)$ and $(2.10)$, can also be represented with

$$
\begin{aligned}
& \psi_{1}(\mathbf{Z})= \begin{cases}1 & \text { if } E[\delta(2 Y-1) \mid \mathbf{Z}]>0 \\
0 & \text { otherwise }\end{cases} \\
& \psi_{0}(\mathbf{X})= \begin{cases}1 & \text { if } E[(1-\delta)(2 Y-1) \mid \mathbf{X}]>0 \\
0 & \text { otherwise. }\end{cases}
\end{aligned}
$$


To appreciate that (2.23) and (2.9) (as well as (2.24) and (2.10)) are equivalent, simply observe that

$$
\begin{aligned}
E(\delta Y \mid \mathbf{Z}) & =E[E(\delta Y \mid \mathbf{Z}, Y) \mid \mathbf{Z}]=E[Y E(\delta \mid \mathbf{Z}, Y) \mid \mathbf{Z}]=E[Y p(\mathbf{Z}, Y) \mid \mathbf{Z}] \\
& =E[Y(Y p(\mathbf{Z}, 1)+(1-Y) p(\mathbf{Z}, 0)) \mid \mathbf{Z}]=E[Y(Y p(\mathbf{Z}, 1))) \mid \mathbf{Z}] \\
& =p_{1}(\mathbf{Z}) \eta_{B}(\mathbf{Z})
\end{aligned}
$$

where, as before, $p_{1}(\mathbf{Z})=p(\mathbf{Z}, 1)$ and $\eta_{B}(\mathbf{Z})=E(Y \mid \mathbf{Z})$. Similarly,

$$
\begin{aligned}
& E(\delta(1-Y) \mid \mathbf{Z})=p_{0}(\mathbf{Z})\left(1-\eta_{B}(\mathbf{Z})\right) \\
& E((1-\delta) Y \mid \mathbf{X})=\left(1-q_{1}(\mathbf{X})\right) \lambda_{B}(\mathbf{X}) \\
& E[(1-\delta)(1-Y) \mid \mathbf{X})=\left(1-q_{0}(\mathbf{X})\right)\left(1-\lambda_{B}(\mathbf{X})\right)
\end{aligned}
$$

Now, the proposed kernel classifier, which essentially replaces the conditional expectations in (2.23) and (2.24), by their corresponding kernel regression estimators, works as follows. Let $\mathcal{K}: \mathcal{R}^{p} \rightarrow \mathcal{R}$ and $\mathcal{H}: \mathcal{R}^{d} \rightarrow \mathcal{R}$ be two given functions (kernels) and put

$$
\begin{aligned}
& \widetilde{g}_{n, 1}(\mathbf{z})= \begin{cases}1 & \text { if } \sum_{i=1}^{n} \delta_{i}\left(2 Y_{i}-1\right) \mathcal{K}\left(\frac{\mathbf{z}_{i}-\mathbf{z}}{h_{n, \mathbf{z}}}\right)>0 \\
0 & \text { otherwise, }\end{cases} \\
& \widetilde{g}_{n, 0}(\mathbf{x})= \begin{cases}1 & \text { if } \sum_{i=1}^{n}\left(1-\delta_{i}\right)\left(2 Y_{i}-1\right) \mathcal{H}\left(\frac{\mathbf{x}_{i}-\mathbf{x}}{h_{n, \mathbf{x}}}\right)>0 \\
0 & \text { otherwise, }\end{cases}
\end{aligned}
$$

where $h_{n, \mathbf{z}}$ and $h_{n, \mathbf{x}}$ are smoothing parameters of the kernels $\mathcal{K}$ and $\mathcal{H}$ respectively. Then our kernel-based estimator of $\psi_{B}$ is defined by

$$
\psi_{n}^{\mathrm{ker}}(\mathbf{Z}, \delta)=\delta \widetilde{g}_{n, 1}(\mathbf{Z})+(1-\delta) \widetilde{g}_{n, 0}(\mathbf{X})
$$

To study the performance of $\psi_{n}^{\text {ker }}$, we assume that the kernels chosen are regular, in the sense of Devroye and Krzyzak (1989). Regular kernel is defined in Definition 
1.3.2. Let $L_{n}\left(\psi_{n}^{\text {ker }}\right)=P\left\{\psi_{n}^{\text {ker }}(\mathbf{Z}, \delta) \neq Y \mid \mathcal{D}_{n}\right\}$ and $L\left(\psi_{B}\right)=P\left\{\psi_{B}(\mathbf{Z}, \delta) \neq Y\right\}$. Then the following result, in conjunction with the Borel-Cantelli lemma, implies that $L_{n}\left(\psi_{n}^{\text {ker }}\right) \stackrel{\text { a.s. }}{\rightarrow} L\left(\psi_{B}\right)$.

Theorem 2.5.1 Let $\psi_{n}^{\text {ker }}$ be as in(2.29) and assume that $\mathcal{K}$ and $\mathcal{H}$ are regular kernels. If $h_{n, \mathbf{z}} \bigvee h_{n, \mathbf{x}} \rightarrow 0$ and $\left(h_{n, \mathbf{z}}^{p} \wedge h_{n, \mathbf{x}}^{d}\right) n \rightarrow \infty$, as $n \rightarrow \infty$, then for every $\epsilon>0$ there is an integer $n_{0}>0$ such that for every $n>n_{0}$

$$
P\left\{L_{n}\left(\psi_{n}^{k e r}\right)-L\left(\psi_{B}\right)>\epsilon\right\} \leqslant 2 e^{-n \epsilon^{2} / 128\left(\rho_{1} \vee \rho_{2}\right)^{2}}
$$

where $\rho_{1}$ and $\rho_{2}$ are positive constants depending on $\mathcal{K}$ and $\mathcal{H}$ respectively.

To prove the theorem we first state the following two lemmas

Lemma 2.5.2 let $G_{n}(\mathbf{Z})$ and $H_{n}(\mathbf{X})$ be any sample-based estimators of $E[\delta(2 Y-$ 1) $\mid \mathbf{Z}]$ and $E[(1-\delta)(2 Y-1) \mid \mathbf{X}]$, respectively, and define the classifier

$$
\psi_{n}(\mathbf{Z}, \delta)=\delta I\left\{G_{n}(\mathbf{Z})>0\right\}+(1-\delta) I\left\{H_{n}(\mathbf{X})>0\right\}
$$

Then

$$
\begin{aligned}
L_{n}\left(\psi_{n}\right)-L\left(\psi_{B}\right) \leqslant & E\left[\left|E[\delta(2 Y-1) \mid \mathbf{Z}]-G_{n}(\mathbf{Z})\right| \mid \mathcal{D}_{n}\right] \\
& +E\left[\left|E[(1-\delta)(2 Y-1) \mid \mathbf{X}]-H_{n}(\mathbf{X})\right| \mid \mathcal{D}_{n}\right]
\end{aligned}
$$

where $\psi_{B}$ is as in (2.22). 


\section{Proof.}

By (2.25), (2.26), (2.27) and (2.28)

$$
\begin{aligned}
E[\delta(2 Y-1) \mid \mathbf{Z}] & =E[\delta Y \mid \mathbf{Z}]-E[\delta(1-Y) \mid \mathbf{Z}] \\
& =p_{1}(\mathbf{Z}) \eta_{B}(\mathbf{Z})-p_{0}(\mathbf{Z})\left(1-\eta_{B}(\mathbf{Z})\right) \\
E[(1-\delta)(2 Y-1) \mid \mathbf{X}] & =E[(1-\delta) Y \mid \mathbf{X}]-E[(1-\delta)(1-Y) \mid \mathbf{X}] \\
& =\left(1-q_{1}(\mathbf{X})\right) \lambda_{B}(\mathbf{X})-\left(1-q_{0}(\mathbf{X})\right)\left(1-\lambda_{B}(\mathbf{X})\right)
\end{aligned}
$$

Now, an application of Corollary 2.3.1 completes the proof of the lemma.

Lemma 2.5.3 (Devroye et al. (1996) Györfi et al. (2002))

Let $\left(U_{i}, \mathbf{V}_{i}\right)_{i=1}^{n}=\mathcal{D}_{n}$ be iid $[-L, L] \times \mathcal{R}^{d}$-valued observations. Put $m(\mathbf{v})=E(U \mid \mathbf{V}=$ $\mathbf{v})$ and define $m_{n}(\mathbf{v})=\sum_{i=1}^{n} U_{i} \mathcal{K}\left(\frac{\mathbf{V}_{i}-\mathbf{v}}{h_{n}}\right) / n E \mathcal{K}\left(\frac{\mathbf{v}-\mathbf{v}}{h_{n}}\right)$, where the kernel $\mathcal{K}(\cdot)$ is regular. If $h_{n} \rightarrow 0$ and $n h_{n}^{d} \rightarrow \infty$, as $n \rightarrow \infty$, then for large $n$,

$$
P\left\{E\left[\left|m_{n}(\mathbf{V})-m(\mathbf{V})\right| \mid \mathcal{D}_{n}\right]>\epsilon\right\} \leqslant e^{-\frac{n \epsilon^{2}}{64 \rho^{2} L}}
$$

where $\rho=\rho(\mathcal{K})$ is a positive constant that depends on $\mathcal{K}$ only.

\section{Proof of Theorem 2.5.1.}

Take

$$
G_{n}(\mathbf{Z})=\frac{\sum_{i=1}^{n} \delta_{i}\left(2 Y_{i}-1\right) \mathcal{K}\left(\frac{\mathbf{z}_{i}-\mathbf{z}}{h_{n, \mathbf{z}}}\right)}{n E \mathcal{K}\left(\frac{\mathbf{z}-\mathbf{z}}{h_{n, \mathbf{z}}}\right)}
$$

and

$$
H_{n}(\mathbf{X})=\frac{\sum_{i=1}^{n}\left(1-\delta_{i}\right)\left(2 Y_{i}-1\right) \mathcal{H}\left(\frac{\mathbf{x}_{i}-\mathbf{x}}{h_{n, \mathbf{x}}}\right)}{n \mathcal{H}\left(\frac{\mathbf{x}-\mathbf{x}}{h_{n, \mathbf{x}}}\right)}
$$


Observe that in view of Lemmas 2.5.2 and 2.5.3,

$$
\begin{aligned}
P\left\{L_{n}\left(\psi_{n}^{\mathrm{ker}}\right)-L\left(\psi_{B}\right)>\epsilon\right\} & \leqslant P\left\{E\left[\left|E[\delta(2 Y-1) \mid \mathbf{Z}]-G_{n}(\mathbf{Z})\right| \mid \mathcal{D}_{n}\right]>\frac{\epsilon}{2}\right\} \\
& +P\left\{E\left[\left|E[(1-\delta)(2 Y-1) \mid \mathbf{X}]-H_{n}(\mathbf{X})\right| \mid \mathcal{D}_{n}\right]>\frac{\epsilon}{2}\right\} \\
& \leqslant e^{-n \epsilon^{2} / 128 \rho_{1}^{2}}+e^{-n \epsilon^{2} / 128 \rho_{2}^{2}} \\
& \leqslant 2 e^{-n \epsilon^{2} / 128\left(\rho_{1} \vee \rho_{2}\right)^{2}}
\end{aligned}
$$

where $\rho_{1}$ and $\rho_{2}$ do not depend on $n$ or $\epsilon$.

The results of this chapter imply that, fully parametric models for classification in the presence of missing covariates are meaningless because of the key role of the missing mechanism. In practice, one does not know the missing probability mechanism $p(\mathbf{X}, \mathbf{V}, Y)$, and it should be estimated via nonparametric models. Furthermore, the nonparametric estimation of $p(\mathbf{X}, \mathbf{V}, Y)$ requires one to impose MAR-type assumptions (which are not necessarily warranted). However, by using our new representation for the optimal classifier, (see, (2.22), (2.23) and (2.24)) one can construct a nonparametric classifier without imposing any assumption on the missing probability mechanism. In the next chapter, we will consider the Swiss-cheese models where missing covariates may appear anywhere among the covariates. 


\section{Chapter 3}

\section{The Best Classifier for the Swiss-Cheese Model}

\subsection{Introduction}

In the previous chapter, we presented the best classifier when a block of covariates was missing (i.e., there was only one missing pattern). However, in practice, there are different patterns for missing covariates with the most general being that the missing covariates may be anywhere in the data set. This realistic model is also called the "Swiss-cheese" model. In this chapter, the main goal is to find the optimal classifier under such models and to propose data-based classifiers without imposing any assumption on the missing probability mechanism. In Section 3.2, we explain the appropriate setup for such models. Section 3.3 is devoted to the derivation of the best classifier for the Swiss-cheese model (in the sense of having the lowest misclassification error rate). In Section 3.4, we construct data-based classifiers in both parametric and nonparametric cases. In the nonparametric case, we are able 
to construct a classifier without imposing any assumption on the missing probability mechanism; this is achieved by first deriving an alternative representation of the optimal classifier which does not depend on $p(\mathbf{x}, \mathbf{v}, y)$ directly. We use kernel regression methods to construct our proposed classifier.

\subsection{General Setup for the Swiss-Cheese Model}

In the most general case, missing values can appear anywhere in the vector of covariates, i.e., the "Swiss-cheese" model. More specifically, corresponding to each covariate vector $\mathbf{Z} \in \mathcal{R}^{p}$, define $\boldsymbol{\delta}=\left(\delta_{1}, \cdots, \delta_{p}\right)^{\prime}$ to be the $p$-dimensional vector such that

$$
\delta_{j}= \begin{cases}0 & \text { if } j^{\text {th }} \text { component of } \mathbf{Z} \text { is missing } \\ 1 & \text { otherwise }\end{cases}
$$

where $j=1, \cdots, p$. There are $2^{p}$ different possible binary vectors of this form; this includes the vector $(0,0, \cdots, 0)$, which corresponds to all components missing, and $(1,1, \cdots, 1)$ which implies that $\mathbf{Z}$ is a complete case. Since, in practice, all possible missing patterns may not be realizable, we suppose that for some $m \in\left\{1,2, \cdots, 2^{p}\right\}$ there are $m$ possible missing patterns. For the $k^{\text {th }}$ pattern $(k=1, \cdots, m)$, let $\mathbf{d}_{k} \in\{0,1\}^{p}$ be its corresponding binary vector. Without loss of generality, if a complete set is observed, we take

$$
\mathbf{d}_{1}=(1,1, \cdots, 1)^{\prime}
$$

and let $\mathbf{d}_{2}, \cdots, \mathbf{d}_{2^{p}}$ be the remaining possible vectors in $\{0,1\}^{p}$, in any order. For each $\mathbf{d}=\left(d_{1}, \cdots, d_{p}\right) \in\{0,1\}^{p}$, let $\mathbf{Z}^{(k)}=\mathbf{Z}\left(\mathbf{d}_{k}\right)$ be the observable part of $\mathbf{Z}$, that is, the $p(k)$-dimensional vector, $(1 \leqslant p(k) \leqslant p)$, obtained from $\mathbf{Z}=\left(Z_{1}, \cdots, Z_{p}\right)$ 
Figure 3.1: Swiss-cheese pattern. Rows correspond to variables, columns to observations. $n=12$

$$
\begin{array}{cccccccccccc}
Z_{1,1} & Z_{1,2} & \star & Z_{1,4} & Z_{1,5} & Z_{1,6} & \star & \star & Z_{1,9} & Z_{1,10} & Z_{1,11} & Z_{1,12} \\
Z_{2,1} & Z_{2,2} & \star & Z_{2,4} & Z_{2,5} & Z_{2,6} & \star & \star & Z_{2,9} & Z_{2,10} & Z_{2,11} & Z_{2,12} \\
\star & \star & Z_{3,3} & Z_{3,4} & \star & Z_{3,6} & Z_{3,7} & Z_{3,8} & Z_{3,9} & Z_{3,10} & \star & Z_{3,12} \\
Z_{4,1} & Z_{4,2} & Z_{4,3} & Z_{4,4} & Z_{4,5} & \star & Z_{4,7} & Z_{4,8} & \star & Z_{4,10} & Z_{4,11} & \star \\
Z_{5,1} & Z_{5,2} & Z_{5,3} & Z_{5,4} & Z_{5,5} & \star & Z_{5,7} & Z_{5,8} & \star & Z_{5,10} & Z_{5,11} & \star \\
\star & \star & Z_{6,3} & Z_{6,4} & Z_{6,5} & Z_{6,6} & Z_{6,7} & Z_{6,8} & Z_{6,9} & Z_{6,10} & Z_{6,11} & Z_{6,12}
\end{array}
$$

upon removing those $Z_{j}$ 's, $1 \leqslant j \leqslant p$, for which $d_{j}$ is 0 . Here

$$
p(k)=\text { number of } 1 \text { 's in } \mathbf{d}_{k} \text {. }
$$

Note that $\mathbf{Z}^{(1)} \equiv \mathbf{Z}\left(\mathbf{d}_{1}\right)=\mathbf{Z}(\mathbf{1})=\mathbf{Z}$ represents a complete case. Thus an observation may be represented by $(\mathbf{Z}, Y, \boldsymbol{\delta})$, where one has to predict the class membership $Y$. Corresponding to this setup, our random sample may be represented by the $n$ i.i.d. triplets

$$
\mathcal{D}_{n}:=\left\{\left(\mathbf{Z}_{1}, Y_{1}, \boldsymbol{\delta}_{1}\right), \cdots,\left(\mathbf{Z}_{n}, Y_{n}, \boldsymbol{\delta}_{n}\right)\right\}
$$

As an example, consider Figure 3.1 where $\mathbf{Z} \in \mathcal{R}^{6}$ and some covariates are missing in a Swiss-cheese model. Here, there are 5 different missing patterns and $\delta_{i} \in\{0,1\}^{6}$ 
and $n=12$. Also

$$
\begin{aligned}
& \boldsymbol{\delta}_{4}=\boldsymbol{\delta}_{10}=\mathbf{d}_{1}=(1,1,1,1,1,1) \\
& \boldsymbol{\delta}_{3}=\boldsymbol{\delta}_{7}=\boldsymbol{\delta}_{8}=\mathbf{d}_{2}=(0,0,1,1,1,1) \\
& \boldsymbol{\delta}_{1}=\boldsymbol{\delta}_{2}=\mathbf{d}_{3}=(1,1,0,1,1,0) \\
& \boldsymbol{\delta}_{5}=\boldsymbol{\delta}_{11}=\mathbf{d}_{4}=(1,1,0,1,1,1) \\
& \boldsymbol{\delta}_{6}=\boldsymbol{\delta}_{9}=\boldsymbol{\delta}_{12}=\mathbf{d}_{5}=(1,1,1,0,0,1) .
\end{aligned}
$$

Suppose that for some $m \in\left\{1,2,3, \cdots, 2^{p}\right\}$ there are $m$ possible missing patterns. To state our results we start by defining the conditional probabilities

$$
p_{k}(\cdot, y)=P\left\{\boldsymbol{\delta}=\mathbf{d}_{k} \mid \mathbf{Z}^{(k)}=\cdot, Y=y\right\}, \quad y=0,1, \quad k=1, \cdots, m
$$

where $p_{1}(\mathbf{z}, y)$ is the conditional probability that $\mathbf{Z}$ is a complete case (i.e., $\boldsymbol{\delta}=$ $\left.\mathbf{d}_{1}=(1, \cdots, 1)^{\prime}\right)$, given $\mathbf{Z}=\mathbf{z}, Y=y$. Here, we have not imposed any MAR-type assumption. In fact, in general, one cannot impose any MAR assumption of the form

$$
P\left\{\boldsymbol{\delta}=\mathbf{d}_{k} \mid \mathbf{Z}, Y\right\}=P\left\{\boldsymbol{\delta}=\mathbf{d}_{k} \mid \widetilde{\mathbf{Z}}, Y\right\},
$$

for some subset $\widetilde{\mathbf{Z}}$ of $\mathbf{Z}$; the reason is that in our very general set-up there is no assumption that some subset of $\mathbf{Z}$, say $\widetilde{\mathbf{Z}}=\left(Z_{1}, Z_{2}\right)$, is always observable. However, if such an assumption is appropriate, then under the MAR mechanism: $P\{\boldsymbol{\delta}=$ $\left.\mathbf{d}_{k} \mid \mathbf{Z}, Y\right\}=P\left\{\boldsymbol{\delta}=\mathbf{d}_{k} \mid Z_{1}, Z_{2}, Y\right\}$. If the covariates are MCAR, then

$$
\mathbf{P}\left(\boldsymbol{\delta}=\mathbf{d}_{k} \mid \mathbf{Z}, Y\right)=P\left(\boldsymbol{\delta}=\mathbf{d}_{k}\right)
$$

In the next section, we use our general setup to derive the classifier with minimum misclassification error rate. 


\subsection{The Best Classifier for the Swiss-Cheese Model}

We have seen under the setup of the last section that an observation may be represented by $(\mathbf{Z}, Y, \boldsymbol{\delta})$, where one has to predict the class membership $Y$. Also note that, we may have $m$ different missing patterns corresponding to $\mathbf{d}_{1}, \cdots, \mathbf{d}_{m}$. If $\delta=\mathbf{d}_{k}, k=1, \cdots, m$, one is confined to classifiers of the form $g_{k}: \mathcal{R}^{p(k)} \rightarrow\{0,1\}$, with $p(k)=\#$ of 1 's in $\mathbf{d}_{k}$. Thus any classifier $\psi$ necessarily has the form

$$
\begin{aligned}
\psi(\mathbf{Z}, \boldsymbol{\delta}) & =\psi_{g_{1}, \cdots, g_{m}}(\mathbf{Z}, \boldsymbol{\delta}) \\
& =I\left\{\boldsymbol{\delta}=\mathbf{d}_{1}\right\} g_{1}\left(\mathbf{Z}^{(1)}\right)+I\left\{\boldsymbol{\delta}=\mathbf{d}_{2}\right\} g_{2}\left(\mathbf{Z}^{(2)}\right)+\cdots+I\left\{\boldsymbol{\delta}=\mathbf{d}_{m}\right\} g_{m}\left(\mathbf{Z}^{(m)}\right) \\
& =\sum_{k=1}^{m} I\left\{\boldsymbol{\delta}=\mathbf{d}_{k}\right\} g_{k}\left(\mathbf{Z}^{(k)}\right)
\end{aligned}
$$

where $\mathbf{Z}^{(k)} \equiv \mathbf{Z}\left(\mathbf{d}_{k}\right)$. Our goal is to present the best classifier. Intuitively, one may use the definition of the best classifier for complete data and propose to take the overall optimal classifier to be as in (3.3) with $g_{k}\left(\mathbf{Z}^{(k)}\right)=I\left\{P\left(Y=1 \mid \mathbf{Z}^{(k)}\right)>\right.$ $\left.\frac{1}{2}\right\}, k=1, \cdots, m$. But this classifier is not optimal in general since the probabilities of the missing data are completely ignored. Because of the crucial role of the missing mechanism, one has to take these missing probabilities into account in order to be able to derive an appropriate classifier in the presence of missing covariates. Now, let

$$
\eta_{k}(\cdot)=P\left\{Y=1 \mid \mathbf{Z}^{(k)}=\cdot\right\}, \quad k=1, \cdots, m
$$

and define the classifier

$$
\psi_{B}(\mathbf{Z}, \boldsymbol{\delta})=\sum_{k=1}^{m} I\left\{\boldsymbol{\delta}=\mathbf{d}_{k}\right\} \psi_{k}\left(\mathbf{Z}^{(k)}\right)
$$


where, for $k=1, \cdots, m$,

$$
\psi_{k}\left(\mathbf{Z}^{(k)}\right)= \begin{cases}1 & \text { if } p_{k 1}\left(\mathbf{Z}^{(k)}\right) \eta_{k}\left(\mathbf{Z}^{(k)}\right)-p_{k 0}\left(\mathbf{Z}^{(k)}\right)\left(1-\eta_{k}\left(\mathbf{Z}^{(k)}\right)\right)>0 \\ 0 & \text { otherwise }\end{cases}
$$

$p_{k 1}\left(\mathbf{Z}^{(k)}\right)=p_{k}\left(\mathbf{Z}^{(k)}, 1\right)$, and $p_{k 0}\left(\mathbf{Z}^{(k)}\right)=p_{k}\left(\mathbf{Z}^{(k)}, 0\right)$. The term $p_{k}\left(\mathbf{Z}^{(k)}, Y\right)$ is defined by (3.2). The following result shows that $\psi_{B}$ is indeed the optimal classifier; it also provides bounds on the performance of $\psi_{B}$.

Theorem 3.3.1 Let $\psi_{B}$ be as in (3.4). Then, for any other classifier $\psi$ given by (3.3), one has:

(i) $L(\psi)-L\left(\psi_{B}\right) \geqslant 0$.

(ii) Suppose that the functions $g_{1}, g_{2}, \cdots, g_{m}$ in (3.3) are of the form

$$
g_{k}\left(\mathbf{Z}^{(k)}\right)=I\left\{G_{k}\left(\mathbf{Z}^{(k)}\right)>0\right\}, k=1, \cdots, m,
$$

for some functions $G_{k}: \mathcal{R}^{p(k)} \rightarrow[-1,1], k=1, \cdots, m$, where $0<p(k)=$ \# of 1 's in $\mathrm{d}_{k}$. Then

$$
L(\psi)-L\left(\psi_{B}\right) \leqslant \sum_{k=1}^{m} E\left|\left[p_{k 1}\left(\mathbf{Z}^{(k)}\right) \eta_{k}\left(\mathbf{Z}^{(k)}\right)-p_{k 0}\left(\mathbf{Z}^{(k)}\right)\left(1-\eta_{k}\left(\mathbf{Z}^{(k)}\right)\right)\right]-G_{k}\left(\mathbf{Z}^{(k)}\right)\right|
$$

Part (i) of Theorem 3.3.1 shows that the classifier defined in (3.4) has the lowest misclassification error. The bound in part (ii) is practically useful when $G_{k}\left(\mathbf{Z}^{(k)}\right)$ is of the form $G_{k}\left(\mathbf{Z}^{(k)}\right)=\widetilde{p}_{k 1}\left(\mathbf{Z}^{(k)}\right) \widetilde{\eta}_{k}\left(\mathbf{Z}^{(k)}\right)-\widetilde{p}_{k 0}\left(\mathbf{Z}^{(k)}\right)\left(1-\widetilde{\eta}_{k}\left(\mathbf{Z}^{(k)}\right)\right)$. Here the functions $\widetilde{p}_{k 1}$ and $\widetilde{p}_{k 0}$ are approximations to $p_{k 1}$ and $p_{k 0}$, and $\widetilde{\eta}_{k}$ is an approximation to $\eta_{k}, k=1, \cdots, m$. If we denote the corresponding classifier by

$$
\widetilde{\psi}(\mathbf{Z}, \boldsymbol{\delta})=\sum_{k=1}^{m} I\left\{\boldsymbol{\delta}=\mathbf{d}_{k}\right\} I\left\{\widetilde{p}_{k 1}\left(\mathbf{Z}^{(k)}\right) \widetilde{\eta}_{k}\left(\mathbf{Z}^{(k)}\right)-\widetilde{p}_{k 0}\left(\mathbf{Z}^{(k)}\right)\left(1-\widetilde{\eta}_{k}\left(\mathbf{Z}^{(k)}\right)\right)>0\right\},
$$


then in view of part (ii) of Theorem 3.3.1, one can study the error difference $L(\widetilde{\psi})-$ $L\left(\psi_{B}\right)$, where $L(\widetilde{\psi})=P\{\widetilde{\psi}(\mathbf{Z}, \boldsymbol{\delta}) \neq Y\}$, by studying the $L_{1}$-differences

$$
\begin{aligned}
& E\left|\widetilde{p}_{k 1}\left(\mathbf{Z}^{(k)}\right)-p_{k 1}\left(\mathbf{Z}^{(k)}\right)\right|, \quad E\left|\widetilde{p}_{k 0}\left(\mathbf{Z}^{(k)}\right)-p_{k 0}\left(\mathbf{Z}^{(k)}\right)\right|, \quad \text { and } \\
& E\left|\widetilde{\eta}_{k}\left(\mathbf{Z}^{(k)}\right)-\eta_{k}\left(\mathbf{Z}^{(k)}\right)\right|, \quad k=1, \cdots, m
\end{aligned}
$$

\section{Proof of Theorem 3.3.1.}

(i). First note that for any classifier $\psi$,

$$
\begin{aligned}
P\{\psi(\mathbf{Z}, \boldsymbol{\delta})=Y\}= & \sum_{k=1}^{m}\left[P\left\{g_{k}\left(\mathbf{Z}^{(k)}\right)=1, Y=1, \boldsymbol{\delta}=\mathbf{d}_{\mathbf{k}}\right\}\right. \\
& \left.+P\left\{g_{k}\left(\mathbf{Z}^{(k)}\right)=0, Y=0, \boldsymbol{\delta}=\mathbf{d}_{\mathbf{k}}\right\}\right] \\
= & \sum_{k=1}^{m}\left[P_{k 1}+P_{k 0}\right], \quad \text { (say.) }
\end{aligned}
$$

But, for $k=1, \cdots, m$,

$$
\begin{aligned}
P_{k 1} & =E[I\left\{g_{k}\left(\mathbf{Z}^{(k)}\right)=1\right\} I\{Y=1\} \underbrace{P\left\{\boldsymbol{\delta}=\mathbf{d}_{k} \mid \mathbf{Z}^{(k)}, Y\right\}}_{p_{k}\left(\mathbf{Z}^{(k)}, Y\right)}] \\
& =E\left[I\left\{g_{k}\left(\mathbf{Z}^{(k)}\right)=1\right\} Y\left(Y p_{k}\left(\mathbf{Z}^{(k)}, 1\right)+(1-Y) p_{k}\left(\mathbf{Z}^{(k)}, 0\right)\right)\right] \\
& =E\left[I\left\{g_{k}\left(\mathbf{Z}^{(k)}\right)=1\right\} Y p_{k 1}\left(\mathbf{Z}^{(k)}\right)\right] \\
& \left.=E\left[I\left\{g_{k}\left(\mathbf{Z}^{(k)}\right)=1\right\} \eta_{k}\left(\mathbf{Z}^{(k)}\right) p_{k 1}\left(\mathbf{Z}^{(k)}\right)\right], \text { (upon conditioning on } \mathbf{Z}^{(k)}\right)
\end{aligned}
$$

where, as before, $p_{k 1}\left(\mathbf{Z}^{(k)}\right):=p_{k}\left(\mathbf{Z}^{(k)}, 1\right)=P\left\{\boldsymbol{\delta}=\mathbf{d}_{k} \mid \mathbf{Z}^{(k)}, Y=1\right\}$. Similarly, for $k=1, \cdots, m$,

$$
P_{k 0}=E\left[I\left\{g_{k}\left(\mathbf{Z}^{(k)}\right)=0\right\}\left(1-\eta_{k}\left(\mathbf{Z}^{(k)}\right)\right) p_{k 0}\left(\mathbf{Z}^{(k)}\right)\right]
$$


where $p_{k 0}\left(\mathbf{Z}^{(k)}\right)=P\left\{\boldsymbol{\delta}=\mathbf{d}_{k} \mid \mathbf{Z}^{(k)}, Y=0\right\}$. Therefore

$$
\begin{aligned}
& L(\psi)-L\left(\psi_{B}\right)= P\left\{\psi_{B}(\mathbf{Z}, \boldsymbol{\delta})=Y\right\}-P\{\psi(\mathbf{Z}, \boldsymbol{\delta})=Y\} \\
&= \sum_{k=1}^{m} E\left[\eta_{k}\left(\mathbf{Z}^{(k)}\right) p_{k 1}\left(\mathbf{Z}^{(k)}\right)\left(I\left\{\psi_{k}\left(\mathbf{Z}^{(k)}\right)=1\right\}-I\left\{g_{k}\left(\mathbf{Z}^{(k)}\right)=1\right\}\right)\right. \\
&\left.+\left(1-\eta_{k}\left(\mathbf{Z}^{(k)}\right)\right) p_{k 0}\left(\mathbf{Z}^{(k)}\right)\left(I\left\{\psi_{k}\left(\mathbf{Z}^{(k)}\right)=0\right\}-I\left\{g_{k}\left(\mathbf{Z}^{(k)}\right)=0\right\}\right)\right] \\
&= \sum_{k=1}^{m} E\left[\left(\eta_{k}\left(\mathbf{Z}^{(k)}\right) p_{k 1}\left(\mathbf{Z}^{(k)}\right)-\left(1-\eta_{k}\left(\mathbf{Z}^{(k)}\right)\right) p_{k 0}\left(\mathbf{Z}^{(k)}\right)\right)\right. \\
&\left.\times\left(I\left\{\psi_{k}\left(\mathbf{Z}^{(k)}\right)=1\right\}-I\left\{g_{k}\left(\mathbf{Z}^{(k)}\right)=1\right\}\right)\right] \\
&= \sum_{k=1}^{m} E\left[\left|\eta_{k}\left(\mathbf{Z}^{(k)}\right) p_{k 1}\left(\mathbf{Z}^{(k)}\right)-\left(1-\eta_{k}\left(\mathbf{Z}^{(k)}\right)\right) p_{k 0}\left(\mathbf{Z}^{(k)}\right)\right|\right. \\
& \geqslant 0 \\
&\left.\times I\left\{\psi_{k}\left(\mathbf{Z}^{(k)}\right) \neq g_{k}\left(\mathbf{Z}^{(k)}\right)\right\}\right]
\end{aligned}
$$

(ii). From part (i), $L(\psi)-L\left(\psi_{B}\right)=$ far r.h.s. of (3.6). By considering the two cases $\left\{\psi_{k}\left(\mathbf{Z}^{(k)}\right)=1, g_{k}\left(\mathbf{Z}^{(k)}\right)=0\right\}$ and $\left\{\psi_{k}\left(\mathbf{Z}^{(k)}\right)=0, g_{k}\left(\mathbf{Z}^{(k)}\right)=1\right\}$ separately, where $g_{k}\left(\mathbf{Z}^{(k)}\right)=I\left\{G_{k}\left(\mathbf{Z}^{(k)}\right)>0\right\}$, it is straightforward to see that the $k^{\text {th }}$ term of the sum on the far r.h.s. of (3.6) is bounded by

$$
E\left|\left[\eta_{k}\left(\mathbf{Z}^{(k)}\right) p_{k 1}\left(\mathbf{Z}^{(k)}\right)-\left(1-\eta_{k}\left(\mathbf{Z}^{(k)}\right)\right) p_{k 0}\left(\mathbf{Z}^{(k)}\right)\right]-G_{k}\left(\mathbf{Z}^{(k)}\right)\right|
$$

and this completes the proof.

The classifier $\psi_{B}$, defined via (3.4) and (3.5), is not applicable in practice for the simple reason that the functions $p_{k}\left(\mathbf{Z}^{(k)}, Y\right)=P\left\{\boldsymbol{\delta}=\mathbf{d}_{k} \mid \mathbf{Z}^{(k)}, Y\right\}$, or the functions $\eta_{k}\left(\mathbf{Z}^{(k)}\right)=P\left\{Y=1 \mid \mathbf{Z}^{(k)}\right\}$, (or both) are virtually always unknown. In the next section, we derive consistent data-based classifiers with minimum assumptions on the missing probability mechanism for both parametric and nonparametric cases. 


\subsection{Data-based Classifiers for the Swiss-Cheese Model}

Given the sample $\mathcal{D}_{n}$ in (3.1), let $G_{n k}\left(\mathbf{Z}^{(k)}\right)$ be any sample version (estimate) of the function $p_{k 1}\left(\mathbf{Z}^{(k)}\right) \eta_{k}\left(\mathbf{Z}^{(k)}\right)-p_{k 0}\left(\mathbf{Z}^{(k)}\right)\left(1-\eta_{k}\left(\mathbf{Z}^{(k)}\right)\right)$ that appears in (3.5). Put

$$
g_{n k}\left(\mathbf{Z}^{(k)}\right)=I\left\{G_{n k}\left(\mathbf{Z}^{(k)}\right)>0\right\}
$$

and define the data-based classifier

$$
\psi_{n}(\mathbf{Z}, \boldsymbol{\delta})=\sum_{k=1}^{m} I\left\{\boldsymbol{\delta}=\mathbf{d}_{k}\right\} g_{n k}\left(\mathbf{Z}^{(k)}\right)
$$

Then the following result, which is a simple corollary to part (ii) of theorem 3.3.1, provides the necessary tools to establish various consistency results.

Corollary 3.4.1 Let $\psi_{B}$ be as in (3.4). Then, for the classifier $\psi_{n}$ defined above,

$$
\begin{aligned}
L_{n}\left(\psi_{n}\right)-L\left(\psi_{B}\right) \leqslant & \sum_{k=1}^{m} E\left(\mid\left[p_{k 1}\left(\mathbf{Z}^{(k)}\right) \eta_{k}\left(\mathbf{Z}^{(k)}\right)-p_{k 0}\left(\mathbf{Z}^{(k)}\right)\left(1-\eta_{k}\left(\mathbf{Z}^{(k)}\right)\right)\right]\right. \\
& \left.-G_{n k}\left(\mathbf{Z}^{(k)}\right) \|\left.\right|_{\mathcal{D}_{n}}\right)
\end{aligned}
$$

where $L\left(\psi_{B}\right)=P\left\{\psi_{B}(\mathbf{Z}, \boldsymbol{\delta}) \neq Y\right\}$ and $L_{n}\left(\psi_{n}\right)=P\left\{\psi_{n}(\mathbf{Z}, \boldsymbol{\delta}) \neq Y \mid \mathcal{D}_{n}\right\}$

Note that, the above corollary is particulary useful in bounding the error difference $L_{n}\left(\psi_{n}\right)-L\left(\psi_{B}\right)$, when $G_{n k}\left(\mathbf{Z}^{(k)}\right)$ is of the form $\widehat{p}_{k 1}\left(\mathbf{Z}^{(k)}\right) \widehat{\eta}_{k}\left(\mathbf{Z}^{(k)}\right)-\widehat{p}_{k 0}\left(\mathbf{Z}^{(k)}\right)(1-$ $\left.\widehat{\eta}_{k}\left(\mathbf{Z}^{(k)}\right)\right)$, for some estimators $\widehat{p}_{k 1}(\cdot), \widehat{p}_{k 0}(\cdot)$, and $\widehat{\eta}_{k}(\cdot)$ of $p_{k 1}(\cdot), p_{k 0}(\cdot)$, and $\eta_{k}(\cdot)$. In 
such cases one only needs to study the $L_{1}$-differences, because

$$
\begin{aligned}
L_{n}\left(\psi_{n}\right)-L\left(\psi_{B}\right) \leqslant & \sum_{k=1}^{m} E\left(\mid\left[p_{k 1}\left(\mathbf{Z}^{(k)}\right) \eta_{k}\left(\mathbf{Z}^{(k)}\right)-p_{k 0}\left(\mathbf{Z}^{(k)}\right)\left(1-\eta_{k}\left(\mathbf{Z}^{(k)}\right)\right)\right]\right. \\
& \left.-\left[\widehat{p}_{k 1}\left(\mathbf{Z}^{(k)}\right) \widehat{\eta}_{k}\left(\mathbf{Z}^{(k)}\right)-\widehat{p}_{k 0}\left(\mathbf{Z}^{(k)}\right)\left(1-\widehat{\eta}_{k}\left(\mathbf{Z}^{(k)}\right)\right)\right]|| \mathcal{D}_{n}\right) \\
\leqslant & \sum_{k=1}^{m} E\left[\left|\widehat{p}_{k 1}\left(\mathbf{Z}^{(k)}\right)-p_{k 1}\left(\mathbf{Z}^{(k)}\right)\right| \mid \mathcal{D}_{n}\right] \\
& +\sum_{k=1}^{m} E\left[\left|\widehat{p}_{k 0}\left(\mathbf{Z}^{(k)}\right)-p_{k 0}\left(\mathbf{Z}^{(k)}\right)\right| \mid \mathcal{D}_{n}\right] \\
& +2 \sum_{k=1}^{m} E\left[\left|\widehat{\eta}_{k}\left(\mathbf{Z}^{(k)}\right)-\eta_{k}\left(\mathbf{Z}^{(k)}\right)\right| \mid \mathcal{D}_{n}\right]
\end{aligned}
$$

In what follows, we consider two methods of estimation. The first method is parametric and deals with the situation where the underlying distribution of $\mathbf{Z}$ is known, up to a vector of unknown parameters. The second method is fully nonparametric. In both cases we shall make the realistic assumption that the functional form of the missing probability mechanism, $p_{k 1}\left(\mathbf{Z}^{(k)}\right)=P\left\{\boldsymbol{\delta}=\mathbf{d}_{k} \mid \mathbf{Z}^{(k)}, Y=1\right\}$ and $p_{k 0}\left(\mathbf{Z}^{(k)}\right)=P\left\{\boldsymbol{\delta}=\mathbf{d}_{k} \mid \mathbf{Z}^{(k)}, Y=0\right\}, k=1, \cdots, m$, is completely unknown and hence $\widehat{p}_{k 1}\left(\mathbf{Z}^{(k)}\right)$ and $\widehat{p}_{k 0}\left(\mathbf{Z}^{(k)}\right)$ will be nonparametric estimates.

\subsubsection{Parametric Estimation}

Suppose that the distribution of $\mathbf{Z}$ is known but the parameters of the distribution are unknown. It is straightforward to show that

$$
\eta_{k, \boldsymbol{\theta}}\left(\mathbf{Z}^{(k)}\right)=\frac{f_{k 1}\left(\mathbf{Z}^{(k)}, \boldsymbol{\theta}_{1}\right) p}{f_{k 1}\left(\mathbf{Z}^{(k)}, \boldsymbol{\theta}_{1}\right) p+f_{k 0}\left(\mathbf{Z}^{(k)}, \boldsymbol{\theta}_{0}\right)(1-p)}
$$

where $f_{k y}\left(\mathbf{Z}^{(k)}, \boldsymbol{\theta}_{y}\right)$ is the density of the subvector $\mathbf{Z}^{(k)}$ for class $y=0,1$ and $p=$ 
$P\{Y=1\}$, and the parameter vector $\boldsymbol{\theta}=\left(\boldsymbol{\theta}_{1}, \boldsymbol{\theta}_{0}, p\right)$. Now, one can re-write (3.5) as

$$
\psi_{k \boldsymbol{\theta}}\left(\mathbf{Z}^{(k)}\right)= \begin{cases}1 & \text { if } p_{k 1}\left(\mathbf{Z}^{(k)}\right) \eta_{k, \boldsymbol{\theta}}\left(\mathbf{Z}^{(k)}\right)-p_{k 0}\left(\mathbf{Z}^{(k)}\right) \eta_{k, \boldsymbol{\theta}}\left(\mathbf{Z}^{(k)}\right)>0 \\ 0 & \text { otherwise }\end{cases}
$$

where $p_{k y}\left(\mathbf{Z}^{(k)}\right)=P\left\{\boldsymbol{\delta}=\mathbf{d}_{k} \mid \mathbf{Z}^{(k)}, Y=y\right\}, y=0,1$. Now (3.8) suggests using plug-in classifiers of the form:

$$
\psi_{n}^{\mathrm{par}}(\mathbf{Z}, \boldsymbol{\delta})=\sum_{k=1}^{m} I\left\{\boldsymbol{\delta}=\mathbf{d}_{k}\right\} \psi_{k, \widehat{\theta}}\left(\mathbf{Z}^{(k)}\right)
$$

with

$$
\psi_{k, \widehat{\boldsymbol{\theta}}}\left(\mathbf{Z}^{(k)}\right)= \begin{cases}1 & \text { if } \widehat{p}_{k 1}\left(\mathbf{Z}^{(k)}\right) \eta_{k, \widehat{\boldsymbol{\theta}}}\left(\mathbf{Z}^{(k)}\right)>\widehat{p}_{k 0}\left(\mathbf{Z}^{(k)}\right) \eta_{k, \widehat{\boldsymbol{\theta}}}\left(\mathbf{Z}^{(k)}\right) \\ 0 & \text { otherwise }\end{cases}
$$

where $\widehat{p}=n^{-1} \sum_{i=1}^{n} Y_{i}, \widehat{\boldsymbol{\theta}}=\left(\widehat{\boldsymbol{\theta}}_{1}, \widehat{\boldsymbol{\theta}}_{0}, \widehat{p}\right)$ is a consistent estimator of $\boldsymbol{\theta}=\left(\boldsymbol{\theta}_{1}, \boldsymbol{\theta}_{0}, p\right)$ and $\widehat{p}_{k y}\left(\mathbf{Z}^{(k)}\right)$ is a nonparametric estimator of $p_{k y}\left(\mathbf{Z}^{(k)}\right), y=0,1$. In the important case of two multivariate normals, Anderson (1957) gives explicit formulas for the MLEs of $\boldsymbol{\theta}$. For more general distributions, one can refer to Little and Rubin (2002; chapters 8 and 14), where the EM algorithm as well as other methods are discussed. (See, Section 2.4.1 for more details on Anderson formulas and EM method.) On the other hand, the nonparametric estimation of $p_{k y}(\cdot)=P\left\{\boldsymbol{\delta}=\mathbf{d}_{k} \mid \mathbf{Z}^{(k)}=\cdot, Y=y\right\}$ can be problematic unless one can impose further assumptions on the missing probability mechanism. To appreciate this, consider any local averaging estimator:

$$
\widehat{p}_{k y}\left(\mathbf{z}^{(k)}\right)=\sum_{i=1}^{n} \omega_{n i}\left(\mathbf{z}^{(k)}, y\right) I\left\{\boldsymbol{\delta}_{i}=\mathbf{d}_{k}\right\} / \sum_{i=1}^{n} \omega_{n i}\left(\mathbf{z}^{(k)}, y\right),
$$

where $\omega_{n i}\left(\mathbf{z}^{(k)}, y\right)=\omega_{n}\left(\mathbf{z}^{(k)}, y ; \mathbf{Z}_{i}^{(k)}, Y_{i}\right)$ are weight functions. The class of estimators (3.10) contains virtually all popular nonparametric estimators; this includes kernel 
estimators, nearest neighbour estimators, histogram, as well as general partitioning estimators. Taking for example, the weight functions to be

$$
\omega_{n i}\left(\mathbf{z}^{(k)}, y\right)=I\left\{Y_{i}=y\right\} \mathcal{H}_{k}\left(\frac{\mathbf{Z}_{i}^{(k)}-\mathbf{z}^{(k)}}{h_{k}}\right)
$$

where $\mathcal{H}_{k}: \mathcal{R}^{p(k)} \rightarrow \mathcal{R}$ is the $k^{\text {th }}$ kernel function with smoothing parameter $h_{k}$ and $p(k)=\#$ of 1 's in $\mathbf{d}_{k}$, results in kernel-type estimators

$$
\begin{aligned}
\widehat{p}_{k 1}\left(\mathbf{z}^{(k)}\right) & =\frac{\sum_{i=1}^{n} Y_{i} I\left\{\boldsymbol{\delta}_{i}=\mathbf{d}_{k}\right\} \mathcal{H}_{k}\left(\frac{\mathbf{z}_{i}^{(k)}-\mathbf{z}^{(k)}}{h_{k}}\right)}{\sum_{i=1}^{n} Y_{i} \mathcal{H}_{k}\left(\frac{\mathbf{z}_{i}^{(k)}-\mathbf{z}^{(k)}}{h_{k}}\right)} \\
\widehat{p}_{k 0}\left(\mathbf{z}^{(k)}\right) & =\frac{\sum_{i=1}^{n}\left(1-Y_{i}\right) I\left\{\boldsymbol{\delta}_{i}=\mathbf{d}_{k}\right\} \mathcal{H}_{k}\left(\frac{\mathbf{z}_{i}^{(k)}-\mathbf{z}^{(k)}}{h_{k}}\right)}{\sum_{i=1}^{n}\left(1-Y_{i}\right) \mathcal{H}_{k}\left(\frac{\mathbf{z}_{i}^{(k)}-\mathbf{z}^{(k)}}{h_{k}}\right)}
\end{aligned}
$$

Unfortunately, these popular estimators are not readily computable for every $k$. To see this, take $k=1$ and observe that the denominator of the r.h.s. of (3.10) is simply

$$
\sum_{i: \delta_{i}=\mathbf{d}_{1}} \omega_{n i}\left(\mathbf{z}^{(1)}, y\right)+\sum_{i: \delta_{i} \neq \mathbf{d}_{1}} \omega_{n i}\left(\mathbf{z}^{(1)}, y\right)
$$

where $\mathbf{z}^{(1)}=\mathbf{z}\left(\right.$ since $\left.\mathbf{d}_{1}=(1,1, \cdots, 1)^{\prime}\right)$. Clearly, the second sum above is not computable $\left(\omega_{n i}\right.$ is not available when $\left.\boldsymbol{\delta}_{i} \neq \mathbf{d}_{1}\right)$. Fortunately, this situation can be rectified if the following MAR-type assumption holds. Suppose that some subset of $\mathbf{Z}$, say $\mathbf{Z}^{*}=\left(Z_{2}, Z_{5}\right)^{\prime}$, is always observable. Then, since $\mathbf{Z}^{*} \in \mathbf{Z}^{(k)}$ for every $k=1, \cdots, m$ (recall that $\mathbf{Z}^{(k)}$ is the observable part/components of $\mathbf{Z}$ under the $k^{\text {th }}$ missing pattern), the natural extension of the MAR assumption to $m$ missing 
patterns requires

$$
\begin{aligned}
p_{k}\left(\mathbf{Z}^{(k)}, Y\right) & =P\left\{\boldsymbol{\delta}=\mathbf{d}_{k} \mid \mathbf{Z}^{(k)}, Y\right\} \\
& \stackrel{\text { MAR }}{=} P\left\{\boldsymbol{\delta}=\mathbf{d}_{k} \mid \mathbf{Z}^{*}, Y\right\} \\
& :=q_{k}\left(\mathbf{Z}^{*}, Y\right), \quad \text { (say) }, k=1, \cdots, m,
\end{aligned}
$$

where $\mathbf{Z}^{(1)} \equiv \mathbf{Z}$. In this case one can estimate $q_{k}\left(\mathbf{z}^{*}, y\right)$, (equivalently $p_{k}\left(\mathbf{z}^{(k)}, y\right)$ ) by

$$
\widehat{q}_{k}\left(\mathbf{z}^{*}, y\right)=\frac{\sum_{i=1}^{n} \omega_{n i}^{*}\left(\mathbf{z}^{*}, y\right) I\left\{\boldsymbol{\delta}_{i}=\mathbf{d}_{k}\right\}}{\sum_{i=1}^{n} \omega_{n i}^{*}\left(\mathbf{z}^{*}, y\right)}, y=0,1
$$

Observe that when $m=2$ (one missing pattern only) the above definition coincides with the usual MAR assumption. The following theorem shows that, $\psi_{n}^{\mathrm{par}}(\mathbf{Z}, \boldsymbol{\delta})$ defined in (3.9) is Bayes consistent.

Theorem 3.4.2 Suppose $\widehat{\boldsymbol{\theta}} \stackrel{P}{\rightarrow} \boldsymbol{\theta}$ and that $\eta_{k, \boldsymbol{\theta}}\left(\mathbf{Z}^{(k)}\right)$ is continuous in $\boldsymbol{\theta}$ with respect to the $L_{1}$ metric. Then $\psi_{n}^{\text {par }}$, as defined in (3.9), is Bayes consistent in the $L_{1}$ sense, i.e.,

$$
E\left[L_{n}\left(\psi_{n}^{p a r}\right)\right] \rightarrow L\left(\psi_{B}\right)
$$

provided that $E\left[\left|\widehat{p}_{k y}\left(\mathbf{Z}^{(k)}\right)-p_{k y}\left(\mathbf{Z}^{(k)}\right)\right| \mid \mathcal{D}_{n}\right] \stackrel{P}{\rightarrow} 0, y=0,1, k=1, \cdots m$.

\section{Proof.}

If $\eta_{k, \boldsymbol{\theta}}\left(\mathbf{Z}^{(k)}\right), k=1, \cdots m$ is continuous in the $L_{1}$ metric and $\widehat{\boldsymbol{\theta}} \stackrel{P}{\rightarrow} \boldsymbol{\theta}$, then

$$
E\left[\left|\eta_{k, \hat{\boldsymbol{\theta}}}\left(\mathbf{Z}^{(k)}\right)-\eta_{k, \boldsymbol{\theta}}\left(\mathbf{Z}^{(k)}\right)\right| \mid \mathcal{D}_{n}\right] \stackrel{P}{\rightarrow} 0, \quad k=1, \cdots m
$$


In view of 3.7 and the dominated convergence theorem

$$
\begin{aligned}
E\left[L_{n}\left(\psi_{n}^{\mathrm{par}}\right)-L\left(\psi_{B}\right)\right] \leqslant & \sum_{k=1}^{m} E\left[\left|\widehat{p}_{k 1}\left(\mathbf{Z}^{(k)}\right)-p_{k 1}\left(\mathbf{Z}^{(k)}\right)\right|\right] \\
& +\sum_{k=1}^{m} E\left[\left|\widehat{p}_{k 0}\left(\mathbf{Z}^{(k)}\right)-p_{k 0}\left(\mathbf{Z}^{(k)}\right)\right|\right] \\
& +2 \sum_{k=1}^{m} E\left[\left|\eta_{k, \widehat{\boldsymbol{\theta}}}\left(\mathbf{Z}^{(k)}\right)-\eta_{k, \boldsymbol{\theta}}\left(\mathbf{Z}^{(k)}\right)\right|\right] .
\end{aligned}
$$

It is worth noting that the parametric approaches require knowing the underlying distribution of $\mathbf{Z}$, as well as imposing restrictive assumptions on the missing probability mechanism. In practice the validity of these assumptions is in doubt. It would be of interest to construct nonparametric classifiers without imposing any assumptions on the missing mechanism. In the next section, we derive a new representation for the optimal classifier defined in (3.4) and (3.5). This representation is then used to construct a "good" classifier based on the kernel regression methods, (good in the sense that it is computable without an MAR-type assumption, it does not depend on the underlying distribution of data, and it is almost surely consistent).

\subsubsection{Nonparametric Estimation}

Suppose that the underlying distribution of the data is completely unknown and/or no assumptions can be made about the missing probability mechanism (i.e., we may have a Not Missing At Random situation). To construct nonparametric classifiers,

we start by giving an equivalent representation of $\psi_{B}$, where $\psi_{B}$ is as in (3.4) and 
(3.5). First observe that:

$$
\begin{aligned}
E\left[Y I\left\{\boldsymbol{\delta}=\mathbf{d}_{k}\right\} \mid \mathbf{Z}^{(k)}\right] & =E[Y \underbrace{E\left(I\left\{\boldsymbol{\delta}=\mathbf{d}_{k}\right\} \mid \mathbf{Z}^{(k)}, Y\right)}_{p_{k}\left(\mathbf{Z}^{(k)}, Y\right)} \mid \mathbf{Z}^{(k)}] \\
& =E\left[Y\left(Y p_{k}\left(\mathbf{Z}^{(k)}, 1\right)+(1-Y) p_{k}\left(\mathbf{Z}^{(k)}, 0\right)\right) \mid \mathbf{Z}^{(k)}\right] \\
& =E\left[Y p_{k 1}\left(\mathbf{Z}^{(k)}\right) \mid \mathbf{Z}^{(k)}\right], \text { where } p_{k 1}\left(\mathbf{Z}^{(k)}\right)=p_{k}\left(\mathbf{Z}^{(k)}, 1\right) \\
& =p_{k 1}\left(\mathbf{Z}^{(k)}\right) \eta_{k}\left(\mathbf{Z}^{(k)} .\right.
\end{aligned}
$$

Similarly,

$$
E\left[(1-Y) I\left\{\boldsymbol{\delta}=\mathbf{d}_{k}\right\} \mid \mathbf{Z}^{(k)}\right]=p_{k 0}\left(\mathbf{Z}^{(k)}\right)\left(1-\eta_{k}\left(\mathbf{Z}^{(k)}\right)\right),
$$

where $p_{k 0}\left(\mathbf{Z}^{(k)}\right)=p_{k}\left(\mathbf{Z}^{(k)}, 0\right)$. Therefore, we have the equivalent representation of $\psi_{B}:$

$$
\psi_{B}(\mathbf{Z}, \boldsymbol{\delta})=\sum_{k=1}^{m} I\left\{\boldsymbol{\delta}=\mathbf{d}_{k}\right\} \psi_{k}\left(\mathbf{Z}^{(k)}\right)
$$

where

$$
\psi_{k}\left(\mathbf{Z}^{(k)}\right)= \begin{cases}1 & \text { if } E\left[Y I\left\{\boldsymbol{\delta}=\mathbf{d}_{k}\right\} \mid \mathbf{Z}^{(k)}\right]-E\left[(1-Y) I\left\{\boldsymbol{\delta}=\mathbf{d}_{k}\right\} \mid \mathbf{Z}^{(k)}\right]>0 \\ 0 & \text { otherwise. }\end{cases}
$$

Clearly, if the regression functions $r_{1}\left(\mathbf{z}^{(k)}\right):=E\left[Y I\left\{\boldsymbol{\delta}=\mathbf{d}_{k}\right\} \mid \mathbf{Z}^{(k)}=\mathbf{z}^{(k)}\right]$ and $r_{0}\left(\mathbf{z}^{(k)}\right):=E\left[(1-Y) I\left\{\boldsymbol{\delta}=\mathbf{d}_{k}\right\} \mid \mathbf{Z}^{(k)}\right]$ are replaced by some estimators $\widehat{r}_{1}\left(\mathbf{z}^{(k)}\right)$ and $\widehat{r}_{0}\left(\mathbf{z}^{(k)}\right)$ in $(3.15)$, then the resulting sample version of $(3.15), \psi_{n k}\left(\mathbf{Z}^{(k)}\right)$ say, can be substituted for $\psi_{k}\left(\mathbf{Z}^{(k)}\right)$ in (3.14) to construct the sample-based classifier $\psi_{n}(\mathbf{Z}, \boldsymbol{\delta})=\sum_{i=1}^{n} I\left\{\boldsymbol{\delta}=\mathbf{d}_{k}\right\} \psi_{k n}\left(\mathbf{Z}^{(k)}\right)$. To carry out the construction of $\psi_{n}(\mathbf{Z}, \boldsymbol{\delta})$ in a fully nonparametric way, (that is, to replace $r_{1}$ and $r_{0}$ by some nonparametric estimators $\widehat{r}_{1}$ and $\widehat{r}_{0}$ ), we propose to use local-averaging methods. More specifically, consider the kernel-based classifier 


$$
\widetilde{\widetilde{\psi}}_{n}(\mathbf{Z}, \boldsymbol{\delta})=\sum_{k=1}^{m} I\left\{\boldsymbol{\delta}=\mathbf{d}_{k}\right\} \widetilde{\psi}_{n, k}\left(\mathbf{Z}^{(k)}\right)
$$

where

$$
\widetilde{\psi}_{n, k}\left(\mathbf{z}^{(k)}\right)=\left\{\begin{aligned}
1 & \text { if } \sum_{i=1}^{n} I\left\{\boldsymbol{\delta}_{i}=\mathbf{d}_{k}\right\} Y_{i} \mathcal{K}_{k}\left(\frac{\mathbf{z}_{i}^{(k)}-\mathbf{z}^{(k)}}{h_{n, k}}\right) \\
& >\sum_{i=1}^{n} I\left\{\boldsymbol{\delta}_{i}=\mathbf{d}_{k}\right\}\left(1-Y_{i}\right) \mathcal{K}_{k}\left(\frac{\mathbf{z}_{i}^{(k)}-\mathbf{z}^{(k)}}{h_{n, k}}\right) \\
0 & \text { otherwise. }
\end{aligned}\right.
$$

Here $\mathcal{K}_{k}: \mathcal{R}^{p(k)} \rightarrow \mathcal{R}$ is the $k^{\text {th }}$ kernel, where $p(k)=\#$ of 1 's in $\mathbf{d}_{k}$. In passing we also note that upon dividing both sides of the inequality in (3.17) by $\sum_{i=1}^{n} \mathcal{K}_{k}\left(\left(\mathbf{Z}_{i}^{(k)}-\right.\right.$ $\left.\mathbf{z}^{(k)}\right) / h_{n, k}$ ), [a term which is not necessarily computable for each $k$ (take, for example, $k=1)], \widetilde{\psi}_{n, k}\left(\mathbf{z}^{(k)}\right)$ reduces to the usual kernel regression estimator for $\psi_{k}\left(\mathbf{z}^{(k)}\right)$ in (3.15). However, the fact that the term $\sum_{i=1}^{n} \mathcal{K}_{k}\left(\left(\mathbf{Z}_{i}^{(k)}-\mathbf{z}^{(k)}\right) / h_{n, k}\right)$ need not be evaluated to compute $\widetilde{\psi}_{n, k}\left(\mathbf{z}^{(k)}\right)$ makes the classifier $\widetilde{\widetilde{\psi}}_{n}$ particularly appealing. How good is $\widetilde{\widetilde{\psi}}_{n}$ ? The following performance bounds of the following theorem in conjunction with the Borel-Cantelli lemma, immediately yields

$$
L_{n}\left(\widetilde{\widetilde{\psi}}_{n}\right) \stackrel{\text { a.s. }}{\rightarrow} L\left(\psi_{B}\right), \text { as } n \rightarrow \infty
$$

where $L_{n}(\widetilde{\widetilde{\psi}})=P\left\{\widetilde{\widetilde{\psi}}(\mathbf{Z}, \boldsymbol{\delta}) \neq Y \mid \mathcal{D}_{n}\right\}$

Theorem 3.4.3 Let $\widetilde{\widetilde{\psi}}_{n}$ be as defined by (3.16) and (3.17). Assume that $\mathcal{K}_{1}, \cdots, \mathcal{K}_{m}$ are regular kernels. If $\bigvee_{k=1}^{m} h_{n, k} \rightarrow 0$ and $\left(\bigwedge_{k=1}^{m} h_{n, k}^{p(k)}\right) n \rightarrow \infty$, as $n \rightarrow \infty$, where $p(k)=\#$ of 1's in $\mathbf{d}_{k}$, then for every $\epsilon>0$ there is an integer $n_{0}>0$ such that for every $n>n_{0}$

$$
P\left\{L_{n}\left(\widetilde{\widetilde{\psi}}_{n}\right)-L\left(\psi_{B}\right)>\epsilon\right\} \leqslant m e^{-c_{2} n \epsilon^{2}}
$$

here the constant $c_{2}>0$ does not depend on $n$ or $\epsilon$. 
To prove the theorem we first state some lemmas.

Lemma 3.4.4 Let $H_{n k}\left(\mathbf{z}^{(k)}\right)$ be any sample-based estimator of $E\left[(2 Y-1) I\left\{\boldsymbol{\delta}=\mathbf{d}_{k}\right\} \mid \mathbf{Z}^{(k)}=\mathbf{z}^{(k)}\right], k=1,2, \cdots, m$, and define the classifier

$$
\psi_{n}(\mathbf{Z}, \boldsymbol{\delta})=\sum_{k=1}^{m} I\left\{\boldsymbol{\delta}=\mathbf{d}_{k}\right\} I\left\{H_{n k}\left(\mathbf{Z}^{(k)}\right)>0\right\}
$$

Then

$$
L_{n}\left(\psi_{n}\right)-L\left(\psi_{B}\right) \leqslant \sum_{k=1}^{m} E\left(\left|E\left[(2 Y-1) I\left\{\boldsymbol{\delta}=\mathbf{d}_{k}\right\} \mid \mathbf{Z}^{(k)}\right]-H_{n k}\left(\mathbf{Z}^{(k)}\right)\right| \mid \mathcal{D}_{n}\right)
$$

where $\psi_{B}$ is as in (3.14).

\section{Proof.}

By (3.12) and (3.13),

$$
E\left[(2 Y-1) I\left\{\boldsymbol{\delta}=\mathbf{d}_{k}\right\} \mid \mathbf{Z}^{(k)}\right]=p_{k 1}\left(\mathbf{Z}^{(k)}\right) \eta_{k}\left(\mathbf{Z}^{(k)}\right)-p_{k 0}\left(\mathbf{Z}^{(k)}\right)\left(1-\eta_{k}\left(\mathbf{Z}^{(k)}\right)\right.
$$

Now, an application of Corollary 3.4.1 completes the proof of the lemma.

Lemma 3.4.5 [Devroye et al. (1996), Györfi et al. (2002)]

Let $\left(U_{i}, \mathbf{V}_{i}\right)_{i=1}^{n}=\mathcal{D}_{n}$ be i.i.d. $[-L, L] \times \mathcal{R}^{d}$-valued observations. Put $m(\mathbf{v})=$ $E(U \mid \mathbf{V}=\mathbf{v})$ and define $m_{n}(\mathbf{v})=\sum_{i=1}^{n} U_{i} \mathcal{K}\left(\frac{\mathbf{v}_{i}-\mathbf{v}}{h_{n}}\right) / n E \mathcal{K}\left(\frac{\mathbf{v}-\mathbf{v}}{h_{n}}\right)$, where the kernel $\mathcal{K}(\cdot)$ is regular. If $h_{n} \rightarrow 0$ and $n h_{n}^{d} \rightarrow \infty$, as $n \rightarrow \infty$, then for large $n$,

$$
P\left\{E\left[\left|m_{n}(\mathbf{V})-m(\mathbf{V})\right| \mid \mathcal{D}_{n}\right]>\epsilon\right\} \leqslant e^{-\frac{n \epsilon^{2}}{64 \rho^{2} L}}
$$

where $\rho=\rho(\mathcal{K})$ is a positive constant that depends on $\mathcal{K}$ only. 


\section{Proof of theorem 3.4.3.}

Take

$$
H_{n k}\left(\mathbf{z}^{(k)}\right)=\frac{\sum_{i=1}^{n} I\left\{\boldsymbol{\delta}_{i}=\mathbf{d}_{k}\right\}\left(2 Y_{i}-1\right) \mathcal{K}_{k}\left(\frac{\mathbf{z}_{i}^{(k)}-\mathbf{z}^{(k)}}{h_{n, k}}\right)}{n E \mathcal{K}_{k}\left(\frac{\mathbf{z}_{i}^{(k)}-\mathbf{z}^{(k)}}{h_{n, k}}\right)}
$$

and observe that in view of Lemmas 3.4.4 and 3.4.5,

$$
\begin{aligned}
P\left\{L_{n}\left(\widetilde{\widetilde{\psi}}_{n}\right)-L\left(\psi_{B}\right)>\epsilon\right\} \leqslant & \sum_{k=1}^{m} P\left\{E \left(\mid E\left[(2 Y-1) I\left\{\boldsymbol{\delta}=\mathbf{d}_{k}\right\} \mid \mathbf{Z}^{(k)}\right]\right.\right. \\
& \left.\left.-H_{n k}^{(k)}\left(\mathbf{Z}^{(k)}\right)|| \mathcal{D}_{n}\right)>\frac{\epsilon}{m}\right\} \\
\leqslant & m e^{-\frac{n \epsilon^{2}}{64 m^{2}\left(\rho_{1} \vee \rho_{2} \vee \cdots \vee \rho_{m}\right)^{2}}}
\end{aligned}
$$

where $\rho_{k}=\rho\left(\mathcal{H}_{k}\right), k=1, \cdots, m$ are positive constants depending on $\mathcal{H}_{k}$ only.

In this chapter, we have considered the realistic case that missing covariates may appear anywhere in the data set. Also, we have constructed parametric and nonparametric classifiers that are consistent under minimal conditions. In the next chapter, we apply partitioning rules for classification when a block of covariates is missing. 


\section{Chapter 4}

\section{Partitioning Rules in the Presence of Missing Covariates}

\subsection{Introduction}

Many important nonparametric classification rules partition the space of observations into cells and then compute statistics locally within each cell. In this chapter, we study a cubic histogram rule and also general partitioning rules in the presence of missing data. In the simplest case, these rules partition the space into cubes or cells whose sizes and positions depend on the number of available data points $n$, but not the data itself. In general, partitioning estimates are computationally superior to the other nonparametric estimates due to the fact that they work by simple indicator functions. Note that, in partitioning rules, one should only know the estimate for each nonempty cell and obviously the number of nonempty cells is much smaller than $n$. This chapter is organized as follows: In the first section, we study the cubic histogram rule when there is only one missing pattern in the data 
and establish strong consistency results for the proposed rule. Section 4.3 presents general sufficient conditions for the almost-sure consistency of our cubic histogram classifier for the Swiss-cheese model. In Section 4.4, we study the fixed partitioning rule (in the sense that the partition does not depend on the data) when a block of covariates is missing, and establish the strong consistency of this rule. In the last section we study the strong consistency of partitioning rule for the Swiss-cheese model.

\subsection{The Regular Histogram Rule When a Block of Covariates is Missing}

In this section we study the cubic histogram rule when there is one missing pattern in the covariates (see Figure 2.1). We recall that when there are no missing data, the histogram rule partitions $\mathcal{R}^{p}$ into cubes of the same size and gives a decision according to the majority vote among $Y_{i}$ 's whose corresponding $\mathbf{Z}_{i}$ falls in the same cube as z. More formally, let $\mathcal{A}_{n}=\left\{A_{n 1}, A_{n 2}, \cdots\right\}$ be a partition of $\mathcal{R}^{p}$ into cubes of size $\ell_{n}>0$ and for each $\mathbf{z} \in \mathcal{R}^{p}$, let $A_{n}(\mathbf{z})$ denotes the cell of $\mathcal{A}_{n}$ containing $\mathbf{z}$. The histogram rule is defined as

$$
\psi_{n}(\mathbf{z})= \begin{cases}1 & \text { if } \sum_{i=1}^{n} I\left\{\mathbf{Z}_{i} \in A_{n}(\mathbf{z})\right\} I\left\{Y_{i}=1\right\}>\sum_{i=1}^{n} I\left\{\mathbf{Z}_{i} \in A_{n}(\mathbf{z})\right\} I\left\{Y_{i}=0\right\} \\ 0 & \text { otherwise }\end{cases}
$$

This means that the histogram rule makes the decision according to the number of zeros and ones among the $Y_{i}$ 's for which $\mathbf{Z}_{i}$ belongs to the same cube into which $\mathbf{z}$ 
falls. According to Theorem 1.3.4 in Chapter 1, the histogram rule defined in (4.1) is strongly consistent if $\ell_{n} \rightarrow 0$ and $n \ell_{n}^{p} \rightarrow \infty$, as $n \rightarrow \infty$. That is, $L_{n}\left(\psi_{n}\right) \stackrel{\text { a.s. }}{\rightarrow} L\left(\psi_{B}\right)$, as $n \rightarrow \infty$ where $L_{n}\left(\psi_{n}\right)=P\left\{\psi_{n}(\mathbf{Z}) \neq Y \mid \mathcal{D}_{n}\right\}$. This theorem was proved by Devroye and Györfi (1983).

Now consider the case where a subset of $\mathbf{Z}$, say $\mathbf{V}$, may be missing (recall $\mathbf{Z}^{\prime}=$ $\left(\mathbf{X}^{\prime}, \mathbf{V}^{\prime}\right)$, where $\mathbf{X} \in \mathcal{R}^{d}$ and $\left.\mathbf{V} \in \mathcal{R}^{p-d}\right)$. In Chapter 2, we proved that the following rule is optimal when a block of covariates is missing (see Theorem 2.2.1 and (2.22), (2.23), and (2.24)):

$$
\psi_{B}(\mathbf{Z}, \delta)=\delta \psi_{1}(\mathbf{Z})+(1-\delta) \psi_{0}(\mathbf{X})
$$

where

$$
\delta= \begin{cases}1 & \text { if } \mathbf{V} \text { is observed } \\ 0 & \text { otherwise }\end{cases}
$$

and

$$
\begin{aligned}
& \psi_{1}(\mathbf{Z})= \begin{cases}1 & \text { if } E[\delta Y \mid \mathbf{Z}]>E[\delta(1-Y) \mid \mathbf{Z}] \\
0 & \text { otherwise }\end{cases} \\
& \psi_{0}(\mathbf{X})= \begin{cases}1 & \text { if } E[(1-\delta) Y \mid \mathbf{X}]>E[(1-\delta)(1-Y) \mid \mathbf{X}] \\
0 & \text { otherwise. }\end{cases}
\end{aligned}
$$

Now, one needs to estimate $\psi_{1}(\mathbf{Z})$ and $\psi_{0}(\mathbf{X})$ based on the given data

$$
\mathcal{D}_{n}=\left\{\left(\mathbf{X}_{1}, \mathbf{V}_{1}, Y_{1}, \delta_{1}\right), \cdots,\left(\mathbf{X}_{n}, \mathbf{V}_{n}, Y_{n}, \delta_{n}\right)\right\}
$$

Let $\mathcal{A}_{n}=\left\{A_{n 1}, A_{n 2}, \cdots\right\}$ be a partition of $\mathcal{R}^{p}$ into cubes of size $\ell_{n, \mathbf{z}}>0$, that is into sets of the type $\prod_{i=1}^{p}\left[k_{i} \ell_{n, \mathbf{z}},\left(k_{i}+1\right) \ell_{n, \mathbf{z}}\right]$, where the $k_{i}$ 's are integers. Similarly, let 
$\mathcal{B}_{n}=\left\{B_{n 1}, B_{n 2}, \cdots\right\}$ be a partition of $\mathcal{R}^{d}$ into cubes of size $\ell_{n, \mathbf{x}}>0$. Then we have the histogram classifier

$$
\psi_{n}^{\mathrm{hist}}(\mathbf{Z}, \delta)=\delta \psi_{n, 1}^{\mathrm{hist}}(\mathbf{Z})+(1-\delta) \psi_{n, 0}^{\mathrm{hist}}(\mathbf{X})
$$

where

$$
\begin{aligned}
& \psi_{n, 1}^{\text {hist }}(\mathbf{z})= \begin{cases}1 & \text { if } \sum_{i=1}^{n} \delta_{i} Y_{i} I\left\{\mathbf{Z}_{i} \in A_{n}(\mathbf{z})\right\}>\sum_{i=1}^{n} \delta_{i}\left(1-Y_{i}\right) I\left\{\mathbf{Z}_{i} \in A_{n}(\mathbf{z})\right\} \\
0 & \text { otherwise }\end{cases} \\
& \psi_{n, 0}^{\text {hist }}(\mathbf{x})=\left\{\begin{array}{cc}
1 & \text { if } \sum_{i=1}^{n}\left(1-\delta_{i}\right) Y_{i} I\left\{\mathbf{X}_{i} \in B_{n}(\mathbf{x})\right\} \\
& >\sum_{i=1}^{n}\left(1-\delta_{i}\right)\left(1-Y_{i}\right) I\left\{\mathbf{X}_{i} \in B_{n}(\mathbf{x})\right\}
\end{array}\right. \\
& 0 \text { otherwise. }
\end{aligned}
$$

In (4.3), $A_{n}(\mathbf{z})=A_{n i}$ if $\mathbf{z} \in A_{n i}$, where $\mathbf{z} \in \mathcal{R}^{p}$. Similarly, in (4.4), $B_{n}(\mathbf{x})=B_{n i}$ if $\mathbf{x} \in B_{n i}$, where $\mathbf{x} \in \mathcal{R}^{d}$. To study the performance of $\psi_{n}^{\text {hist }}$, we first state the following result. In what follows $L_{n}\left(\psi_{n}^{\text {hist }}\right)=P\left\{\psi_{n}^{\text {hist }}(\mathbf{Z}, \delta) \neq Y \mid \mathcal{D}_{n}\right\}$ and $L\left(\psi_{B}\right)=$ $P\left\{\psi_{B}(\mathbf{Z}, \delta) \neq Y\right\}$

Theorem 4.2.1 Let $\psi_{n}^{\text {hist }}$ be as defined in (4.2). Assume that the sequence of partitions $\left\{\mathcal{A}_{n}\right\}$ and $\left\{\mathcal{B}_{n}\right\}$ satisfy the following two conditions as $n \rightarrow \infty: \ell_{n, \mathbf{z}} \bigvee \ell_{n, \mathbf{x}} \rightarrow 0$ and $\left(\ell_{n, \mathbf{z}}^{p} \wedge \ell_{n, \mathbf{x}}^{d}\right) n \rightarrow \infty$. Then, for every $\epsilon>0$, there is an integer $n_{0}>0$ such that for every $n>n_{0}$,

$$
P\left\{L_{n}\left(\psi_{n}^{\text {hist }}\right)-L\left(\psi_{B}\right)>\epsilon\right\} \leqslant 2 e^{-\frac{n \epsilon^{2}}{128}}
$$

The performance bounds of Theorem 4.2.1 in conjunction with the Borel-Cantelli lemma immediately yields 


$$
L_{n}\left(\psi_{n}^{\text {hist }}\right) \stackrel{\text { a.s. }}{\rightarrow} L\left(\psi_{B}\right), \quad \text { as } n \rightarrow \infty
$$

The proof of the above theorem is based on the following key result.

Lemma 4.2.2 (Devroye and Györfi (1983), Györfi et al. (2002)) Let $\left(U_{i}, \mathbf{V}_{i}\right)_{i=1}^{n}=\mathcal{D}_{n}$ be i.i.d. $\quad[-L, L] \times \mathcal{R}^{d}$-valued observations and $\mathcal{P}_{n}=$ $\left\{P_{n 1}, P_{n 2}, \cdots\right\}$ be a cubic partition, where the cells $P_{n i}$ are cubes of size $h_{n}>0$. Put $m(\mathbf{v})=E(U \mid \mathbf{V}=\mathbf{v})$ and $m_{n}(\mathbf{v})=\sum_{i=n}^{n} U_{i} I\left\{\mathbf{V}_{i} \in P_{n}(\mathbf{v})\right\} / n P\left\{\mathbf{V} \in P_{n}(\mathbf{v})\right\}$ where $P_{n}(\mathbf{v})=P_{n i}$ if $\mathbf{v} \in P_{n i}$. If $h_{n} \rightarrow 0$ and $n h_{n}^{d} \rightarrow \infty$, as $n \rightarrow \infty$, then for each $\epsilon>0$ and $n$ large enough,

$$
P\left\{E\left[\left|m_{n}(\mathbf{V})-m(\mathbf{V})\right| \mid \mathcal{D}_{n}\right]>\epsilon\right\} \leqslant e^{-\frac{n \epsilon^{2}}{32 L^{2}}}
$$

\section{Proof of Theorem 4.2.1.}

Define

$$
G_{n}(\mathbf{Z})=\frac{\sum_{i=1}^{n} \delta_{i}\left(2 Y_{i}-1\right) I\left\{\mathbf{Z}_{i} \in A_{n}(\mathbf{z})\right\}}{n P\left\{\mathbf{Z} \in A_{n}(\mathbf{z})\right\}}
$$

and

$$
H_{n}(\mathbf{X})=\frac{\sum_{i=1}^{n}\left(1-\delta_{i}\right)\left(2 Y_{i}-1\right) I\left\{\mathbf{X}_{i} \in B_{n}(\mathbf{x})\right\}}{n P\left\{\mathbf{X} \in B_{n}(\mathbf{x})\right\}}
$$

where $0 / 0=0$ by definition. Now, one may rewrite the classifier defined in (4.2) as

$$
\psi_{n}^{\text {hist }}(\mathbf{Z}, \delta)=\delta I\left\{G_{n}(\mathbf{Z})>0\right\}+(1-\delta) I\left\{H_{n}(\mathbf{X})>0\right\}
$$

In view of Lemma 2.5.2, 


$$
\begin{aligned}
L_{n}\left(\psi_{n}^{\text {hist }}\right)-L\left(\psi_{B}\right) \leqslant & E\left[\left|E[\delta(2 Y-1) \mid \mathbf{Z}]-G_{n}(\mathbf{Z})\right| \mid \mathcal{D}_{n}\right] \\
& +E\left[\left.\left|E[(1-\delta)(2 Y-1) \mid \mathbf{X}]-H_{n}(\mathbf{X})\right|\right|_{\left.\mathcal{D}_{n}\right]}\right.
\end{aligned}
$$

Then

$$
\begin{aligned}
P\left\{L_{n}\left(\psi_{n}^{\text {hist }}\right)-L\left(\psi_{B}\right)>\epsilon\right\} & \leqslant P\left\{E\left[\left|E[\delta(2 Y-1) \mid \mathbf{Z}]-G_{n}(\mathbf{Z})\right| \mid \mathcal{D}_{n}\right]>\frac{\epsilon}{2}\right\} \\
& +P\left\{E\left[\left|E[(1-\delta)(2 Y-1) \mid \mathbf{X}]-H_{n}(\mathbf{X})\right| \mid \mathcal{D}_{n}\right]>\frac{\epsilon}{2}\right\} .
\end{aligned}
$$

Apply Lemma 4.2.2 to bound each term on the right-hand side of (4.7), one obtains

$$
P\left\{L_{n}\left(\psi_{n}^{\text {hist }}\right)-L\left(\psi_{B}\right)>\epsilon\right\} \leqslant 2 e^{-\frac{n \epsilon^{2}}{128}}
$$

This completes the proof of Theorem 4.2.1.

It is important to mention that the above result does not require any MAR-type assumptions and continues to hold for the general NMAR case as well. The proposed histogram rule in (4.2) along with (4.3) and (4.4) is a strongly consistent classifier in the case that a block of covariates is missing. However, in practice, we may be confronted with the general case where the missing values appear anywhere in the vector of covariates. In the next section, we present a histogram rule for the full Swiss-cheese model. 


\subsection{The Regular Histogram Rule for the Swiss- Cheese Model}

In the previous section we proposed a histogram rule that was appropriate in the presence of one missing pattern in the vector of covariates. In what follows we extend these results to the Swiss-cheese model with $m$ possible missing patterns, where $m \in\left\{1,2,3, \cdots, 2^{p}\right\}$. See Section 3.2 for more details. To state our main results, let

$$
p_{k}(\cdot, y)=P\left\{\boldsymbol{\delta}=\mathbf{d}_{k} \mid \mathbf{Z}^{(k)}=\cdot, Y=y\right\}, \quad y=0,1, \quad k=1, \cdots, m,
$$

and recall that (Section 3.3) the best classifier for the Swiss-cheese model is given by

$$
\psi_{B}(\mathbf{Z}, \boldsymbol{\delta})=\sum_{k=1}^{m} I\left\{\boldsymbol{\delta}=\mathbf{d}_{k}\right\} \psi_{k}\left(\mathbf{Z}^{(k)}\right)
$$

where $\psi_{k}\left(\mathbf{Z}^{(k)}\right): \mathcal{R}^{p(k)} \rightarrow\{0,1\}$, with $p(k)=\#$ of 1 's in $\mathbf{d}_{k}$, is defined by

$$
\psi_{k}\left(\mathbf{Z}^{(k)}\right)= \begin{cases}1 & \text { if } p_{k 1}\left(\mathbf{Z}^{(k)}\right) \eta_{k}\left(\mathbf{Z}^{(k)}\right)-p_{k 0}\left(\mathbf{Z}^{(k)}\right)\left(1-\eta_{k}\left(\mathbf{Z}^{(k)}\right)\right)>0 \\ 0 & \text { otherwise }\end{cases}
$$

Here, $\eta_{k}(\cdot)=P\left\{Y=1 \mid \mathbf{Z}^{(k)}=\cdot\right\}, p_{k 1}\left(\mathbf{Z}^{(k)}\right)=p_{k}\left(\mathbf{Z}^{(k)}, 1\right)$, and $p_{k 0}\left(\mathbf{Z}^{(k)}\right)=$ $p_{k}\left(\mathbf{Z}^{(k)}, 0\right), \quad k=1, \cdots, m$, where the term $p_{k}\left(\mathbf{Z}^{(k)}, Y\right)$ is defined by (4.8). Suppose that the underlying distribution of the data is completely unknown and, at the same time, no assumptions can be made about the missing probability mechanism (i.e., we may have the Not Missing At Random situation). To construct "good" 
nonparametric classifiers (good in the sense that it is computable without an MARtype assumption, it does not depend on the underlying distribution of the data, and it is almost surely consistent), we recall the following equivalent representation of $\psi_{k}\left(\mathbf{Z}^{(k)}\right)$, as given by (3.15) in Section 3.4 .2 ,

$$
\psi_{k}\left(\mathbf{Z}^{(k)}\right)= \begin{cases}1 & \text { if } E\left[(2 Y-1) I\left\{\boldsymbol{\delta}=\mathbf{d}_{k}\right\} \mid \mathbf{Z}^{(k)}\right]>0 \\ 0 & \text { otherwise. }\end{cases}
$$

The advantage of (4.11) is that it does not depend on the hard-to-estimate quantities $p_{k y}\left(\mathbf{Z}^{(k)}\right)$ and $\eta_{k}\left(\mathbf{Z}^{(k)}\right)$ directly. To estimate $\psi_{k}\left(\mathbf{Z}^{(k)}\right)$, we shall use the histogram rule which partitions $\mathcal{R}^{p(k)}$ (recall $p(k)=\#$ of 1 's in $\mathbf{d}_{k}$ ), into cubes of the same size and makes a decision according to the majority vote among the $Y_{i}$ 's such that the corresponding $\mathbf{Z}_{i}^{(k)}$ falls in the same cube as $\mathbf{z}^{(k)}$. More precisely, let $\mathcal{A}_{n, k}=$ $\left\{A_{n 1, k}, A_{n 2, k}, \cdots\right\}$ be a partition of $\mathcal{R}^{p(k)}$ into cubes of size $\ell_{n, k}>0$, that is, into sets of the type $\prod_{i=1}^{p(k)}\left[k_{i} \ell_{n, k},\left(k_{i}+1\right) \ell_{n, k}\right]$, where the $k_{i}$ 's are integers. The histogram classifier is defined by

$$
\psi_{n}^{\mathrm{H}}(\mathbf{Z}, \boldsymbol{\delta})=\sum_{i=1}^{m} I\left\{\boldsymbol{\delta}=\mathbf{d}_{k}\right\} \widehat{\psi}_{n, k}\left(\mathbf{Z}^{(k)}\right)
$$

where

$$
\widehat{\psi}_{n, k}\left(\mathbf{Z}^{(k)}\right)= \begin{cases}1 & \text { if } \sum_{i=1}^{n} I\left\{\boldsymbol{\delta}_{i}=\mathbf{d}_{k}\right\}\left(2 Y_{i}-1\right) I\left\{\mathbf{Z}_{i}^{(k)} \in A_{n, k}\left(\mathbf{Z}^{(k)}\right)\right\}>0 \\ 0 & \text { otherwise. }\end{cases}
$$

Here $\mathbf{z}^{(k)} \in \mathcal{R}^{p(k)}$, and $A_{n, k}\left(\mathbf{z}^{(k)}\right)=A_{n i, k}$ if $\mathbf{z}^{(k)} \in A_{n i, k}$. In passing we note that upon dividing both sides of the inequality in (4.13) by $\sum_{i=1}^{n} I\left\{\mathbf{Z}_{i}^{(k)} \in A_{n, k}\left(\mathbf{z}^{(k)}\right)\right\}$, (a term which is not necessarily computable for each $k$ ), $\widehat{\psi}_{n, k}\left(\mathbf{Z}^{(k)}\right)$ reduces to the 
usual histogram regression estimator for $\psi_{k}\left(\mathbf{Z}^{(k)}\right)$ in (4.11). However, the fact that the term $\sum_{i=1}^{n} I\left\{\mathbf{Z}_{i}^{(k)} \in A_{n, k}\left(\mathbf{z}^{(k)}\right)\right\}$ need not be evaluated to compute $\widehat{\psi}_{n, k}\left(\mathbf{Z}^{(k)}\right)$ makes the classifier $\psi_{n}^{\mathrm{H}}$ particularly appealing. How good is $\psi_{n}^{\mathrm{H}}$ ? The performance bounds of the following theorem in conjunction with the Borel-Cantelli lemma yields

$$
L_{n}\left(\psi_{n}^{\mathrm{H}}\right) \stackrel{a . s .}{\rightarrow} L\left(\psi_{B}\right), \quad n \rightarrow \infty
$$

where $L_{n}\left(\psi_{n}^{\mathbf{H}}\right)=P\left\{\psi_{n}^{\mathrm{H}}(\mathbf{Z}, \boldsymbol{\delta}) \neq Y \mid \mathcal{D}_{n}\right\}$.

Theorem 4.3.1 Let $\psi_{n}^{H}$ be as defined via (4.12) and (4.13). If $\mathcal{A}_{n, 1}, \cdots, \mathcal{A}_{n, m}$ satisfy the following two conditions as $n \rightarrow \infty: \bigvee_{k=1}^{m} \ell_{n, k} \rightarrow 0$ and $\left(\bigwedge_{k=1}^{m} \ell_{n, k}^{p(k)}\right) n \rightarrow \infty$, where $p(k)=\#$ of 1 's in $\mathbf{d}_{k}$, then for every $\epsilon>0$ there is an integer $n_{0}>0$ such that for every $n>n_{0}$

$$
P\left\{L_{n}\left(\psi_{n}^{H}\right)-L\left(\psi_{B}\right)>\epsilon\right\} \leqslant m e^{-\frac{n \epsilon^{2}}{32 m}}
$$

\section{Proof.}

One may write $\psi_{n}^{\mathrm{H}}$ as follow

$$
\psi_{n}^{\mathrm{H}}(\mathbf{Z}, \boldsymbol{\delta})=\sum_{k=1}^{m} I\left\{\boldsymbol{\delta}=\mathbf{d}_{k}\right\} I\left\{H_{n k}\left(\mathbf{Z}^{(k)}\right)>0\right\}
$$

where

$$
H_{n k}\left(\mathbf{z}^{(k)}\right):=\frac{\sum_{i=1}^{n} I\left\{\boldsymbol{\delta}_{i}=\mathbf{d}_{k}\right\}\left(2 Y_{i}-1\right) I\left\{\mathbf{Z}_{i}^{(k)} \in A_{n, k}\left(\mathbf{z}^{(k)}\right)\right\}}{n P\left\{\mathbf{Z}^{(k)} \in A_{n, k}\left(\mathbf{z}^{(k)}\right)\right\}} .
$$

with $0 / 0=0$ by definition. According to Lemma 3.4.4

$$
L_{n}\left(\psi_{n}^{\mathrm{H}}\right)-L\left(\psi_{B}\right) \leqslant \sum_{k=1}^{m} E\left(\left|E\left[(2 Y-1) I\left\{\boldsymbol{\delta}=\mathbf{d}_{k}\right\} \mid \mathbf{Z}^{(k)}\right]-H_{n k}\left(\mathbf{Z}^{(k)}\right)\right| \mid \mathcal{D}_{n}\right)
$$


Now observe that in view of Lemma 4.2.2,

$$
\begin{aligned}
P\left\{L_{n}\left(\psi_{n}^{\mathrm{H}}\right)-L\left(\psi_{B}\right)>\epsilon\right\} \leqslant & \sum_{k=1}^{m} P\left\{E \left(\mid E\left[(2 Y-1) I\left\{\boldsymbol{\delta}=\mathbf{d}_{k}\right\} \mid \mathbf{Z}^{(k)}\right]\right.\right. \\
& \left.\left.-H_{n k}^{(k)}\left(\mathbf{Z}^{(k)}\right)|| \mathcal{D}_{n}\right)>\frac{\epsilon}{m}\right\} \\
\leqslant & m e^{-\frac{n \epsilon^{2}}{32 m^{2}}} .
\end{aligned}
$$

This completes the proof.

In the regular histogram rule, the cells $A_{n i, k}$ are cubes of volume $\ell_{n, k}^{p}$. But instead of cubes of the same size, we may consider rectangles $A_{n i, k}$ with side lengths $\ell_{n 1, k}, \cdots \ell_{n p, k}$. In fact, in the more general case, we can partition $\mathcal{R}^{p}$ into disjoint cells. In the next section, we propose the general partitioning rules in the presence of missing covariates.

\subsection{The General Partitioning Rule When a Block of Covariates is Missing}

In this section we study the general partitioning rules. Recall from Section 1.3.3 that many important classification rules partition $\mathcal{R}^{p}$ into disjoint cells $P_{n 1}, P_{n 2}, \cdots$

and classify in each cell according to the majority vote among the labels of the $\mathbf{Z}_{i}$ 's falling in the cell containing Z. Formally,

$$
g_{n}(\mathbf{z})= \begin{cases}1 & \text { if } \sum_{i=1}^{n} I\left\{Y_{i}=1\right\} I\left\{\mathbf{Z}_{i} \in P_{n}(\mathbf{z})\right\}>\sum_{i=1}^{n} I\left\{Y_{i}=0\right\} I\left\{\mathbf{Z}_{i} \in P_{n}(\mathbf{z})\right\} \\ 0 \text { othewise } & \end{cases}
$$


where $P_{n}(\mathbf{z})$ denotes the cell containing $\mathbf{z}$. This rule requires two conditions for consistency: First, the cells should be small enough so that local changes of the distribution can be detected, i.e., by changing $\mathbf{Z}$, its weights in the neighbourhood will also be changed. On the other hand, cells should be large enough to contain a large number of points so that averaging among the labels is effective, because one may write

$g_{n}(\mathbf{z})= \begin{cases}1 & \text { if } \frac{1}{\sum_{i=1}^{n} I\left\{\mathbf{Z}_{i} \in P_{n}(\mathbf{z})\right\}} \sum_{i: \mathbf{Z}_{i} \in P_{n}(\mathbf{z})} Y_{i}>\frac{1}{\sum_{i=1}^{n} I\left\{\mathbf{Z}_{i} \in P_{n}(\mathbf{z})\right\}} \sum_{i: \mathbf{Z}_{i} \in P_{n}(\mathbf{z})}\left(1-Y_{i}\right) \\ 0 & \text { othewise }\end{cases}$

where $\frac{1}{\sum_{i=1}^{n} I\left\{\mathbf{Z}_{i} \in P_{n}(\mathbf{z})\right\}} \sum_{i: \mathbf{Z}_{i} \in P_{n}(\mathbf{z})} Y_{i}$ is an average among the labels and it is effective if $\sum_{i=1}^{n} I\left\{\mathbf{Z}_{i} \in P_{n}(\mathbf{z})\right\}$ is a large number. More precisely, let

$$
\operatorname{diam}\left(P_{n}\right)=\sup _{\mathbf{z}_{1}, \mathbf{z}_{2} \in P_{n}}\left\|\mathbf{z}_{1}-\mathbf{z}_{2}\right\|
$$

be the diameter of a set $P_{n}$, and let

$$
N(\mathbf{z})=\sum_{i=1}^{n} I\left\{\mathbf{Z}_{i} \in P_{n}(\mathbf{z})\right\}
$$

be the number of $\mathbf{Z}_{i}$ 's falling in the same cell as $\mathbf{z}$. As we saw in Theorem 1.3.3, if

$$
\operatorname{diam}\left(P_{n}(\mathbf{Z})\right) \rightarrow 0 \text { in probability and } N(\mathbf{Z}) \rightarrow \infty \text { in probability }
$$

then

$$
E\left\{L_{n}\left(g_{n}\right)\right\} \rightarrow L\left(\psi_{B}\right)
$$

where $L_{n}\left(g_{n}\right)=P\left\{g_{n}(\mathbf{Z}) \neq Y \mid \mathcal{D}_{n}\right\}$. The two conditions in (4.14) ensure that a random cell selected according to the distribution of $\mathbf{Z}$ has a small diameter, 
and contains many points with large probability. One may show $g_{n}(\mathbf{Z})$ is strongly consistent as follows. First define

$$
\eta_{n}(\mathbf{z})=\frac{\sum_{i=1}^{n} I\left\{Y_{i}=1\right\} I\left\{\mathbf{Z}_{i} \in P_{n}(\mathbf{z})\right\}}{n P\left\{\mathbf{Z} \in P_{n}(\mathbf{z})\right\}} .
$$

Clearly, the decision based on $\eta_{n}$, i.e.,

$$
g_{n}(\mathbf{z})= \begin{cases}1 & \text { if } \frac{\sum_{i=1}^{n} I\left\{Y_{i}=1\right\} I\left\{\mathbf{Z}_{i} \in P_{n}(\mathbf{z})\right\}}{n P\left\{\mathbf{Z} \in P_{n}(\mathbf{z})\right\}}>\frac{\sum_{i=1}^{n} I\left\{Y_{i}=0\right\} I\left\{\mathbf{Z}_{i} \in P_{n}(\mathbf{z})\right\}}{n P\left\{\mathbf{Z} \in P_{n}(\mathbf{z})\right\}} \\ 0 & \text { othewise }\end{cases}
$$

is just the general partition rule. Now, by Corollary 1.4.2,

$$
P\left\{L_{n}\left(g_{n}\right)-L\left(\psi_{B}\right)>\epsilon\right\} \leqslant P\left\{E\left[\left|\eta_{n}(\mathbf{Z})-\eta_{B}(\mathbf{Z})\right| \mid \mathcal{D}_{n}\right]>\frac{\epsilon}{2}\right\}
$$

The following lemma establishes strong consistency for $\eta_{n}$.

Lemma 4.4.1 [Devroye and Györfi (1983), Györfi et al. (2002)]

Let $\left(U_{i}, \mathbf{V}_{i}\right)_{i=1}^{n}=\mathcal{D}_{n}$ be i.i.d. $\quad[-L, L] \times \mathcal{R}^{d}$-valued observations. Put $m(\mathbf{v})=$ $E(U \mid \mathbf{V}=\mathbf{v})$ and define $m_{n}(\mathbf{v})=\sum_{i=1}^{n} U_{i} I\left\{\mathbf{V}_{i} \in A_{n}(\mathbf{v})\right\} / n P\left\{\mathbf{V} \in A_{n}(\mathbf{v})\right\}$, where $A_{n}(\mathbf{v})$ denotes the cell of $\mathcal{A}_{n}=\left\{A_{n 1}, A_{n 2}, \cdots\right\}$ containing $\mathbf{v}$. If for each sphere $S$ centered at the origin

$$
\max _{i: A_{n i} \cap S \neq \varnothing} \operatorname{diam}\left(A_{n i}\right) \rightarrow 0, \text { and } \quad \frac{\left|\left\{i: A_{n i} \cap S \neq \varnothing\right\}\right|}{n} \rightarrow 0 \text {, as } n \rightarrow \infty
$$

then for each $\epsilon>0$ and large $n$,

$$
P\left\{E\left[\left|m_{n}(\mathbf{V})-m(\mathbf{V})\right| \mid \mathcal{D}_{n}\right]>\epsilon\right\} \leqslant e^{-\frac{n \epsilon^{2}}{32 L^{2}}}
$$


Now, consider the case where a block of covariates is missing (recall, $\mathbf{Z}^{\prime}=\left(\mathbf{X}^{\prime}, \mathbf{V}^{\prime}\right)$, where $\mathbf{X} \in \mathcal{R}^{d}$ and $\mathbf{V} \in \mathcal{R}^{p-d}$ ). By Theorem 2.2.1

$$
\psi_{B}(\mathbf{Z}, \delta)=\delta \psi_{\mathbf{1}}(\mathbf{Z})+(1-\delta) \psi_{0}(\mathbf{X})
$$

is the optimal rule, where

$$
\begin{aligned}
& \psi_{1}(\mathbf{Z})= \begin{cases}1 & \text { if } E[\delta(2 Y-1) \mid \mathbf{Z}]>0 \\
0 & \text { otherwise }\end{cases} \\
& \psi_{0}(\mathbf{X})= \begin{cases}1 & \text { if } E[(1-\delta)(2 Y-1) \mid \mathbf{X}]>0 \\
0 & \text { otherwise }\end{cases}
\end{aligned}
$$

(see, (2.23), and (2.24)). Here, the proposed partitioning classifier, which essentially replaces the conditional expectations in (4.15) and (4.16), by their corresponding partitioning regression estimators, works as follows. Let $\mathcal{P}_{n}=\left\{P_{n 1}, P_{n 2}, \cdots\right\}$ be a partition of $\mathcal{R}^{p}$ into disjoint cells and $\mathcal{P}_{n}^{\prime}=\left\{P_{n 1}^{\prime}, P_{n 2}^{\prime}, \cdots\right\}$ be a partition of $\mathcal{R}^{d}$ into disjoint cells and put

$$
\psi_{n, 1}^{\text {part }}(\mathbf{z})= \begin{cases}1 & \text { if } \sum_{i=1}^{n} \delta_{i}\left(2 Y_{i}-1\right) I\left\{\mathbf{Z}_{i} \in P_{n}(\mathbf{z})\right\}>0 \\ 0 & \text { otherwise }\end{cases}
$$

where $P_{n}(\mathbf{z})=P_{n i}$ if $\mathbf{z} \in P_{n i}$ and

$$
\psi_{n, 0}^{\text {part }}(\mathbf{x})= \begin{cases}1 & \text { if } \sum_{i=1}^{n}\left(1-\delta_{i}\right)\left(2 Y_{i}-1\right) I\left\{\mathbf{X}_{i} \in P_{n}^{\prime}(\mathbf{x})\right\}>0 \\ 0 & \text { otherwise }\end{cases}
$$

where $P_{n}^{\prime}(\mathbf{x})=P_{n i}^{\prime}$ if $\mathbf{x} \in P_{n i}^{\prime}$. Then our estimator of $\psi_{B}$ is defined by

$$
\psi_{n}^{\text {part }}(\mathbf{Z}, \delta)=\delta \psi_{n, 1}^{\text {part }}(\mathbf{Z})+(1-\delta) \psi_{n, 0}^{\text {part }}(\mathbf{X})
$$


To study the performance of $\psi_{n}^{\text {part }}$, let $L_{n}\left(\psi_{n}^{\text {part }}\right)=P\left\{\psi_{n}^{\text {part }}(\mathbf{Z}, \delta) \neq Y \mid \mathcal{D}_{n}\right\}$ and $L\left(\psi_{B}\right)=P\left\{\psi_{B}(\mathbf{Z}, \delta) \neq Y\right\}$. The following theorem provides sufficient conditions for the strong consistency of the partitioning rule. The first condition ensures that the cells of the underlying partition shrink to zero inside a bounded set, so the estimate is local in this sense. The second condition means that the number of cells inside a bounded set is small with respect to $n$.

Theorem 4.4.2 Let $\psi_{n}^{\text {part }}$ be as in (4.19). If for each sphere $S_{1}$ and $S_{2}$ centered at origin (in $\mathcal{R}^{p}$ and $\mathcal{R}^{d}$, respectively),

$$
\lim _{n \rightarrow \infty} \max _{i: P_{n i} \cap S_{1} \neq \varnothing} \operatorname{diam}\left(P_{n i}\right) \bigvee \max _{i: P_{n i}^{\prime} \cap S_{2} \neq \varnothing} \operatorname{diam}\left(P_{n i}^{\prime}\right)=0
$$

and

$$
\lim _{n \rightarrow \infty}\left(\left|\left\{i: P_{n i} \cap S_{1} \neq \varnothing\right\}\right| \bigvee\left|\left\{i: P_{n i}^{\prime} \cap S_{2} \neq \varnothing\right\}\right|\right) \frac{1}{n}=0
$$

then for every $\epsilon>0$ there is an integer $n_{0}>0$ such that for every $n>n_{0}$,

$$
P\left\{L_{n}\left(\psi_{n}^{\text {part }}\right)-L\left(\psi_{B}\right)>\epsilon\right\} \leqslant 2 e^{-\frac{n \epsilon^{2}}{128}}
$$

The bounds in Theorem 4.4.2, in conjunction with the Borel-Cantelli lemma, implies that

$$
L_{n}\left(\psi_{n}^{\text {part }}\right) \stackrel{\text { a.s. }}{\rightarrow} L\left(\psi_{B}\right)
$$

Remark 4.4.3 Note that, $\psi_{n, 0}^{\text {part }}(\mathrm{X})$ in (4.19) is based on the partitioning rule as well, though one may classify $\mathbf{X}$ based on any other nonparametric rule such as the 
histogram rule or the kernel rule. Then, the new classifier is

$$
\psi_{n}^{*}(\mathbf{Z}, \delta)=\delta \psi_{n, 1}^{\text {part }}(\mathbf{Z})+(1-\delta) \psi_{n, 0}(\mathbf{X})
$$

where $\psi_{n, 1}^{\text {part }}(\mathbf{Z})$ is defined in (4.17) and $\psi_{n, 0}(\mathbf{X})$, is a data-based estimator for (4.16).

\section{Proof of Theorem 4.4.2.}

Take

$$
G_{n}(\mathbf{z})=\frac{\sum_{i=1}^{n} \delta_{i}\left(2 Y_{i}-1\right) I\left\{\mathbf{Z}_{i} \in P_{n}(\mathbf{z})\right\}}{n P\left\{\mathbf{Z} \in P_{n}(\mathbf{z})\right\}}
$$

and

$$
H_{n}(\mathbf{X})=\frac{\sum_{i=1}^{n}\left(1-\delta_{i}\right)\left(2 Y_{i}-1\right) I\left\{\mathbf{X}_{i} \in P_{n}^{\prime}(\mathbf{x})\right\}}{n P\left\{\mathbf{X} \in P_{n}^{\prime}(\mathbf{x})\right)},
$$

with $0 / 0=0$ by definition. Now, in view of Lemmas 2.5 .2 and 4.4.1,

$$
\begin{aligned}
P\left\{L_{n}\left(\psi_{n}^{\text {part }}\right)-L\left(\psi_{B}\right)>\epsilon\right\} \leqslant & P\left\{E\left[\left|E[\delta(2 Y-1) \mid \mathbf{Z}]-G_{n}(\mathbf{Z})\right| \mid \mathcal{D}_{n}\right]>\frac{\epsilon}{2}\right\} \\
& +P\left\{E\left[\left|E[(1-\delta)(2 Y-1) \mid \mathbf{X}]-H_{n}(\mathbf{X})\right| \mid \mathcal{D}_{n}\right]>\frac{\epsilon}{2}\right\} \\
\leqslant & 2 e^{-\frac{n \epsilon^{2}}{128}}
\end{aligned}
$$

This complete the proof of Theorem 4.4.2.

In this section, we derived strongly consistent estimation for $\psi_{B}$ based on the partitioning rule, when a block of covariates is missing. This is done without imposing any assumptions on the underlying missing probability mechanism. The most general case, where the missing values can appear anywhere in the vector of covariates, will be addressed in the next section. 


\subsection{The General Partitioning Rule for the Swiss- Cheese Model}

In the previous section, we discussed the general partitioning rule when there is one pattern for missing covariates. However, in practice, there can be different missing patterns in the covariates, with the most general being the case where the missing covariates appear anywhere in the data, (the Swiss-cheese model). In Section 3.2, we explained the appropriate setup for the best classifier when we were confronted with such models. In this section we would like to propose general partitioning classifiers without imposing any assumption on the missing probability mechanism. Recall from (4.9) and (4.11) that the best classifier for this model is

$$
\psi_{B}(\mathbf{Z}, \boldsymbol{\delta})=\sum_{k=1}^{m} I\left\{\boldsymbol{\delta}=\mathbf{d}_{k}\right\} \psi_{k}\left(\mathbf{Z}^{(k)}\right)
$$

where

$$
\psi_{k}\left(\mathbf{Z}^{(k)}\right)= \begin{cases}1 & \text { if } E\left[(2 Y-1) I\left\{\boldsymbol{\delta}=\mathbf{d}_{k}\right\} \mid \mathbf{Z}^{(k)}\right]>0 \\ 0 & \text { otherwise. }\end{cases}
$$

Clearly, if the regression function $r\left(\mathbf{z}^{(k)}\right):=E\left[(2 Y-1) I\left\{\boldsymbol{\delta}=\mathbf{d}_{k}\right\} \mid \mathbf{Z}^{(k)}\right]$ is replaced by some estimator $\widehat{r}\left(\mathbf{z}^{(k)}\right)$ in (4.21), then the resulting sample version of (4.21), $\psi_{n k}\left(\mathbf{Z}^{(k)}\right)$ say, can be substituted for $\psi_{k}\left(\mathbf{Z}^{(k)}\right)$ in (4.20) to construct the sample version $\psi_{n}(\mathbf{Z}, \boldsymbol{\delta})=\sum_{k=1}^{m} I\left\{\boldsymbol{\delta}=\mathbf{d}_{k}\right\} \psi_{n k}\left(\mathbf{Z}^{(k)}\right)$. To carry out the construction of $\psi_{n}(\mathbf{Z}, \delta)$ in a fully nonparametric way, (equivalently, to replace $r$ by some nonparametric estimators $\widehat{r}$ ), we propose to use local-averaging methods. More specially, consider the general partitioning rule which partitions $\mathcal{R}^{p(k)}$ (where $p(k)=\#$ of 1 's in $\mathbf{d}_{k}$ ), 
into disjoints cells and makes a decision according to the majority vote among the $Y_{i}$ 's such that the corresponding $\mathbf{Z}_{i}^{(k)}$ falls in the same cells as $\mathbf{z}^{(k)}$. Formally, let $\mathcal{P}_{n, k}=\left\{P_{n 1, k}, P_{n 2, k}, \cdots\right\}$ be a partition of $\mathcal{R}^{p(k)}$ into disjoint cells, then the general partitioning estimator is defined by

$$
\hat{\hat{\psi}}_{n, k}\left(\mathbf{Z}^{(k)}\right)= \begin{cases}1 & \text { if } \sum_{i=1}^{n} I\left\{\boldsymbol{\delta}_{i}=\mathbf{d}_{k}\right\}\left(2 Y_{i}-1\right) I\left\{\mathbf{Z}_{i}^{(k)} \in P_{n, k}\left(\mathbf{z}^{(k)}\right)\right\} \\ 0 & \text { otherwise }\end{cases}
$$

where for every $\mathbf{z}^{(k)} \in \mathcal{R}^{p(k)}, P_{n, k}\left(\mathbf{z}^{(k)}\right)=P_{n i, k}$ if $\mathbf{z}^{(k)} \in P_{n i, k}$. Finally, our proposed partitioning classifier is given by

$$
\psi_{n}^{\mathrm{P}}(\mathbf{Z}, \boldsymbol{\delta})=\sum_{k=1}^{m} I\left\{\boldsymbol{\delta}=\mathbf{d}_{k}\right\} \hat{\hat{\psi}}_{n, k}\left(\mathbf{Z}^{(k)}\right)
$$

How good is $\psi_{n}^{\mathrm{P}}$ ? The performance bounds of the following theorem in conjunction with the Borel-Cantelli lemma yields

$$
L_{n}\left(\psi_{n}^{\mathrm{P}}\right) \stackrel{a . s .}{\rightarrow} L\left(\psi_{B}\right), \quad n \rightarrow \infty
$$

where $L_{n}\left(\psi_{n}^{\mathrm{P}}\right)=P\left\{\psi_{n}^{\mathrm{P}}(\mathbf{Z}, \boldsymbol{\delta}) \neq Y \mid \mathcal{D}_{n}\right\}$

Theorem 4.5.1 Let $\psi_{n}^{P}$ be as defined via (4.23) and (4.22). If for each sphere $S_{1}, \cdots, S_{m}$ centered at origin (in $\mathcal{R}^{p(1)}, \cdots, \mathcal{R}^{p(m)}$, respectively), the sequence of partitions $\mathcal{P}_{n, 1}, \cdots, \mathcal{P}_{n, m}$ satisfy the following two conditions:

$$
\lim _{n \rightarrow \infty} \bigvee_{j=1}^{m} \max _{i: P_{n i, j} \cap S_{j} \neq \varnothing} \operatorname{diam}\left(P_{n i, j}\right)=0
$$

and 


$$
\lim _{n \rightarrow \infty} \bigvee_{j=1}^{m}\left(\left|\left\{i: P_{n i, j} \cap S_{j} \neq \varnothing\right\}\right|\right) \frac{1}{n}=0,
$$

then for every $\epsilon>0$ there is an integer $n_{0}>0$ such that for every $n>n_{0}$

$$
P\left\{L_{n}\left(\psi_{n}^{P}\right)-L\left(\psi_{B}\right)>\epsilon\right\} \leqslant m e^{-\frac{n \epsilon^{2}}{32 m^{2}}}
$$

\section{Proof.}

One may write $\psi_{n}^{\mathrm{P}}$ as follows

$$
\psi_{n}^{\mathrm{P}}(\mathbf{Z}, \boldsymbol{\delta})=\sum_{k=1}^{m} I\left\{\boldsymbol{\delta}=\mathbf{d}_{k}\right\} I\left\{H_{n k}\left(\mathbf{Z}^{(k)}\right)>0\right\}
$$

where

$$
H_{n k}\left(\mathbf{Z}^{(k)}\right):=\frac{\sum_{i=1}^{n} I\left\{\boldsymbol{\delta}_{i}=\mathbf{d}_{k}\right\}\left(2 Y_{i}-1\right) I\left\{\mathbf{Z}_{i}^{(k)} \in P_{n, k}\left(\mathbf{z}^{(k)}\right)\right\}}{n P\left\{\mathbf{Z}^{(k)} \in P_{n, k}\left(\mathbf{z}^{(k)}\right)\right\}},
$$

with $0 / 0=0$ by definition. According to Lemma 3.4.4

$$
L_{n}\left(\psi_{n}^{\mathbf{P}}\right)-L\left(\psi_{B}\right) \leqslant \sum_{k=1}^{m} E\left(\left|E\left[(2 Y-1) I\left\{\boldsymbol{\delta}=\mathbf{d}_{k}\right\} \mid \mathbf{Z}^{(k)}\right]-H_{n k}\left(\mathbf{Z}^{(k)}\right)\right| \mid \mathcal{D}_{n}\right)
$$

Now observe that in view of Lemma 4.4.1,

$$
\begin{aligned}
& P\left\{L_{n}\left(\psi_{n}^{\mathrm{P}}\right)-L\left(\psi_{B}\right)>\epsilon\right\} \\
\leqslant & P\left\{\sum_{k=1}^{m} E\left(\left|E\left[(2 Y-1) I\left\{\boldsymbol{\delta}=\mathbf{d}_{k}\right\} \mid \mathbf{Z}^{(k)}\right]-H_{n k}\left(\mathbf{Z}^{(k)}\right)\right| \mid \mathcal{D}_{n}\right)>\epsilon\right\} \\
\leqslant & \sum_{k=1}^{m} P\left\{E\left(\left|E\left[(2 Y-1) I\left\{\boldsymbol{\delta}=\mathbf{d}_{k}\right\} \mid \mathbf{Z}^{(k)}\right]-H_{n k}\left(\mathbf{Z}^{(k)}\right)\right| \mid \mathcal{D}_{n}\right)>\frac{\epsilon}{m}\right\} \\
\leqslant & m e^{-\frac{n \epsilon^{2}}{3 m^{2}}} .
\end{aligned}
$$


The proof is now completed.

Remark 4.5.2 Partitioning and regular histogram rules are particularly attractive from the computational point of view because these rules are constructed based on simple indicator functions. But, these rules have the undesirable property that they are less accurate at borders of cells of the partition than in the middle of cells. This is because points near the border of a cell should have less weight in a decision regarding the cell's center. To remedy this problem, one may use the kernel methods discussed in Chapters 2 and 3.

We have derived a number of parametric and nonparametric consistent classifier in this chapter as well as Chapters 2 and 3, in the presence of missing covariates. Although, we explained in Chapter 1 that there were several standard methods to deal with missing data, such as imputation or using Horvitz-Thompson estimators, we did not use them in these chapters. These methods are discussed in the next chapter. 


\section{Chapter 5}

\section{The Least-Squares Approach}

\subsection{Introduction}

In the previous chapters, we proposed a number of parametric and nonparametric methods to construct good classification rules. We also studied the theoretical properties of these classifiers. But sometimes, in practice, one is willing to impose a model on $\eta_{B}(\mathbf{z})=P\{Y=1 \mid \mathbf{Z}=\mathbf{z}\}$ in advance. For example $\eta_{B}$ may follow the logistic model, that is $\eta_{B}(\mathbf{z})=e^{b_{0}+b_{1} z_{1}+\cdots+b_{p} z_{p}} /\left(1+e^{b_{0}+b_{1} z_{1}+\cdots+b_{p} z_{p}}\right)$. In general, if one knows that $\eta_{B}$ belongs to a class $\mathcal{M}$ of functions $\eta: \mathcal{R}^{p} \rightarrow[0,1]$, then the least-squares method can be used to pick the empirically best classifier, $\eta_{n}$. That is, $\eta_{n}$ is selected by minimizing the empirical squared error over $\mathcal{M}$. In this chapter, we use this idea to construct classifiers when a block of covariates is missing. The performance of each proposed method is assessed by obtaining exponential bounds on the deviation of its conditional errors from that of the optimal classifier. In Section 5.2 kernel-based imputation is employed to handle the presence of missing covariates under the MAR assumption. We show that the resulting classifier is strongly 
consistent. Section 5.3 is devoted to the use of a Horvitz-Thompson-type inverse weighting approach when the probability of missing covariates is unknown. When the probability of missing covariates is unknown, two methods are proposed to estimate it. The NMAR case is discussed in Section 5.4 and we find a classifier based on minimizing the empirical squared error without imposing MAR-type assumptions.

\subsection{Minimizing the Empirical Squared Error in the MAR Case}

In Section 1.4 the data $\mathcal{D}_{n}:=\left\{\left(\mathbf{Z}_{1}, Y_{1}\right), \cdots,\left(\mathbf{Z}_{n}, Y_{n}\right)\right\}$ were used to select a function $\eta_{n}$ from a class $\mathcal{M}$ of candidate regression functions $\eta: \mathcal{R}^{p} \rightarrow[0,1]$, based on minimizing the empirical squared error over $\mathcal{M}$, that is

$$
\eta_{n}=\underset{\eta \in \mathcal{M}}{\operatorname{argmin}} \frac{1}{n} \sum_{i=1}^{n}\left|Y_{i}-\eta\left(\mathbf{Z}_{i}\right)\right|^{2},
$$

and the corresponding classification rule is

$$
\psi_{n}(\mathbf{z})= \begin{cases}1 & \text { if } \eta_{n}(\mathbf{z})>\frac{1}{2} \\ 0 & \text { otherwise }\end{cases}
$$

Let $L_{n}\left(\psi_{n}\right)=P\left\{\psi_{n}(\mathbf{Z}) \neq Y \mid \mathcal{D}_{n}\right\}$, then $\psi_{n}$ is strongly consistent in the sense that $L_{n}\left(\psi_{n}\right) \stackrel{a . s .}{\rightarrow} L\left(\psi_{B}\right)$, as $n \rightarrow \infty$. For more on this and other related results see Devroye et al. (1996, Chapter 29).

Now one needs to apply this method (minimizing the empirical squared error), when one block of covariates is missing, or in other words, there is one missing pattern for covariates. This case is defined in Section 1.5. (Recall $\mathbf{Z}^{\prime}=\left(\mathbf{X}^{\prime}, \mathbf{V}^{\prime}\right) \in \mathcal{R}^{p}$ where $\mathbf{X} \in \mathcal{R}^{d}$ is always observed and $\mathbf{V} \in \mathcal{R}^{p-d}$ may be missing at random.) We saw in 
Remark 2.2.3 that in the MAR case, one may represent the optimal classifier by

$$
\psi_{B}(\mathbf{Z}, \delta)=\delta \psi_{1}(\mathbf{Z})+(1-\delta) \psi_{0}(\mathbf{X})
$$

where $\psi_{1}: \mathcal{R}^{p} \rightarrow\{0,1\}$ and $\psi_{0}: \mathcal{R}^{d} \rightarrow\{0,1\}$ defined by

$$
\begin{aligned}
& \psi_{1}(\mathbf{Z})= \begin{cases}1 & \text { if } p_{1}(\mathbf{X}) \eta_{B}(\mathbf{Z})-p_{0}(\mathbf{X})\left(1-\eta_{B}(\mathbf{Z})\right)>0 \\
0 & \text { otherwise }\end{cases} \\
& \psi_{0}(\mathbf{X})= \begin{cases}1 & \text { if }\left(1-p_{1}(\mathbf{X})\right) \lambda_{B}(\mathbf{X})-\left(1-p_{0}(\mathbf{X})\right)\left(1-\lambda_{B}(\mathbf{X})\right)>0 \\
0 & \text { otherwise }\end{cases}
\end{aligned}
$$

Recall that in the MAR case

$$
p_{1}(\mathbf{X})=p(\mathbf{X}, 1), p_{0}(\mathbf{X})=p(\mathbf{X}, 0)
$$

where

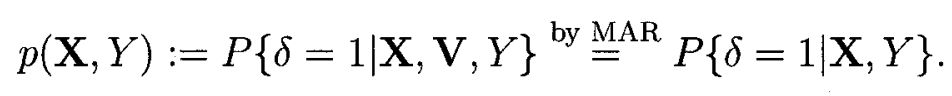

Now, $\eta_{B}(\cdot), \lambda_{B}(\cdot), p_{1}(\cdot)$ and $p_{0}(\cdot)$ should be estimated based on the data. Formally, given the data $\mathcal{D}_{n}:=\left\{\left(\mathbf{Z}_{1}, Y_{1}, \delta_{1}\right), \cdots,\left(\mathbf{Z}_{n}, Y_{n}, \delta_{n}\right)\right\}=\left\{\left(\mathbf{X}_{1}, \mathbf{V}_{1}, Y_{1}, \delta_{1}\right), \cdots,\left(\mathbf{X}_{n}\right.\right.$, $\left.\left.\mathbf{V}_{n}, Y_{n}, \delta_{n}\right)\right\}$, one would like to predict $Y$ corresponding to the new observation $(\mathbf{X}, \mathbf{V}, Y, \delta)$. If $\delta=0$, i.e., if $\mathbf{V}$ is missing, then one can find a Bayes consistent classifier using, for example, the least-squares method discussed earlier, i.e., choose $\lambda_{n}$ :

$$
\lambda_{n}=\underset{\lambda \in \Lambda}{\operatorname{argmin}} \frac{1}{n} \sum_{i=1}^{n}\left|Y_{i}-\lambda\left(\mathbf{X}_{i}\right)\right|^{2},
$$

provided that the class $\Lambda$ contains $\lambda_{B}$, where $\lambda_{B}(\mathbf{x})=P\{Y=1 \mid \mathbf{X}=\mathbf{x}\}$ as before (see (1.8)). Note that, there are of course other popular classification methods that 
one could use instead of the least-squares approach. Also, to estimate $p_{1}(\mathbf{x})$ and $p_{0}(\mathbf{x})$, one may use any popular nonparametric method. The situation however is completely different when $\delta=1$, i.e., when $\mathbf{V}$ (and thus all of $\mathbf{Z}=\left(\begin{array}{l}\mathbf{x} \\ \mathbf{V}\end{array}\right)$ ) is observable in $(\mathbf{X}, \mathbf{V}, Y, \delta)$. Since not every $\mathbf{Z}_{i}$ is completely observable in $\mathcal{D}_{n}$, one might consider a counterpart of (5.1) based on the complete cases, i.e., one would choose $\eta_{n} \in \mathcal{M}$ that minimizes

$$
S_{n}(\eta)=\frac{1}{n_{1}} \sum_{i=1}^{n} \delta_{i}\left|Y_{i}-\eta\left(\mathbf{Z}_{i}\right)\right|^{2}, \text { where } n_{1}=\sum_{i=1}^{n} \delta_{i} .
$$

Unfortunately, there are drawbacks to this approach: In the first place, if a large proportion of the sample (say 75\%) is missing, then by discarding/ignoring the incomplete cases one may be losing useful information which could otherwise be available from all those $\left(\mathbf{X}_{i}, Y_{i}\right)$ 's for which $\delta_{i}=0$. Secondly, the above sum of squares is not (in general) unbiased for $E(Y-\eta(\mathbf{Z}))^{2}$, under the MAR assumption. This implies that the resulting empirical processes (indexed by $\eta \in \mathcal{M}$ ) are not centred, not even asymptotically. The reason for emphasizing this fact is that the resulting function-indexed empirical processes play a crucial role in obtaining exponential bounds on the performance of the resulting classifiers. Such bounds, in conjunction with the Borel-Cantelli lemma, immediately provide various strong consistency results.

In the next sections, a number of procedures will be proposed to "correct" the definition of $S_{n}(\eta)$, under different assumptions.

\subsubsection{A Hypothetical Situation}

To motivate the methods discuss in Section 5.2.2, consider the hypothetical situation where one has available the function $E\left(\left|Y_{i}-\eta\left(\mathbf{Z}_{i}\right)\right|^{2} \mid \mathbf{X}_{i}, Y_{i}\right)$ for every $\eta \in \mathcal{M}$. Re- 
define $S_{n}(\eta)$ to be

$$
\widetilde{S}_{n}(\eta)=\frac{1}{n} \sum_{i=1}^{n}\left[\delta_{i}\left|Y_{i}-\eta\left(\mathbf{Z}_{i}\right)\right|^{2}+\left(1-\delta_{i}\right) E\left(\left|Y_{i}-\eta\left(\mathbf{Z}_{i}\right)\right|^{2} \mid \mathbf{X}_{i}, Y_{i}\right)\right]
$$

The following lemma shows that $\widetilde{S}_{n}(\eta)$ is an unbiased estimator for $E|Y-\eta(\mathbf{Z})|^{2}$.

Lemma 5.2.1 Under the MAR assumption,

$$
E\left(\widetilde{S}_{n}(\eta)\right)=E|Y-\eta(\mathbf{Z})|^{2}
$$

\section{Proof.}

Note that

$E\left(\widetilde{S}_{n}(\eta)\right)=E\left[\left(1-\delta_{i}\right)\left[E\left(\left|Y_{i}-\eta\left(\mathbf{Z}_{i}\right)\right|^{2} \mid \mathbf{X}_{i}, Y_{i}\right)-\left|Y_{i}-\eta\left(\mathbf{Z}_{i}\right)\right|^{2}\right]\right]+E\left|Y_{i}-\eta\left(\mathbf{Z}_{i}\right)\right|^{2}$

On the other hand

$$
\begin{aligned}
& E\left\{E\left[\left(1-\delta_{i}\right)\left[E\left(\left|Y_{i}-\eta\left(\mathbf{Z}_{i}\right)\right|^{2} \mid \mathbf{X}_{i}, Y_{i}\right)-\left|Y_{i}-\eta\left(\mathbf{Z}_{i}\right)\right|^{2}\right] \mid Y_{i}, \mathbf{Z}_{i}\right]\right\} \\
& =E\left\{\left[E\left(\left|Y_{i}-\eta\left(\mathbf{Z}_{i}\right)\right|^{2} \mid \mathbf{X}_{i}, Y_{i}\right)-\left|Y_{i}-\eta\left(\mathbf{Z}_{i}\right)\right|^{2}\right] E\left[\left(1-\delta_{i}\right) \mid Y_{i}, \mathbf{Z}_{i}\right]\right\} \\
& \stackrel{\text { by }}{=}{ }^{\mathrm{MAR}} E\left\{\left[E\left(\left|Y_{i}-\eta\left(\mathbf{Z}_{i}\right)\right|^{2} \mid \mathbf{X}_{i}, Y_{i}\right)-\left|Y_{i}-\eta\left(\mathbf{Z}_{i}\right)\right|^{2}\right] E\left[\left(1-\delta_{i}\right) \mid Y_{i}, \mathbf{X}_{i}\right]\right\} \\
& =E\left\{E\left[\left(1-\delta_{i}\right) \mid Y_{i}, \mathbf{X}_{i}\right] E\left[E\left(\left|Y_{i}-\eta\left(\mathbf{Z}_{i}\right)\right|^{2} \mid \mathbf{X}_{i}, Y_{i}\right)-\left|Y_{i}-\eta\left(\mathbf{Z}_{i}\right)\right|^{2} \mid Y_{i}, \mathbf{X}_{i}\right]\right\} \\
& =0 .
\end{aligned}
$$

This completes the proof.

Let $\widetilde{\eta}_{n}=\underset{\eta \in \mathcal{M}}{\operatorname{argmin}} \widetilde{S}_{n}(\eta)$. Also, let $\lambda_{n}(\mathbf{x})$ be any estimator of $\lambda(\mathbf{x})=P\{Y=1 \mid \mathbf{X}=$ $\mathbf{x}$. Then the resulting plug-in classifier is given by

$$
\widetilde{\psi}_{n}(\mathbf{Z}, \delta)=\delta \widetilde{\psi}_{n, 1}(\mathbf{Z})+(1-\delta) \psi_{n, 0}(\mathbf{X})
$$


where $\widetilde{\psi}_{n, 1}(\mathbf{Z})=I\left\{\widehat{p}_{1}(\mathbf{X}) \widetilde{\eta}_{n}(\mathbf{Z})-\widehat{p}_{0}(\mathbf{X})\left(1-\widetilde{\eta}_{n}(\mathbf{Z})\right)>0\right\}$ and $\psi_{n, 0}(\mathbf{X})=I\{(1-$ $\left.\left.\widehat{p}_{1}(\mathbf{X})\right) \lambda_{n}(\mathbf{X})-\left(1-\widehat{p}_{0}(\mathbf{X})\right)\left(1-\lambda_{n}(\mathbf{X})\right)>0\right\}$. Here, $\widehat{p}_{1}(\mathbf{x})$ and $\widehat{p}_{0}(\mathbf{x})$ are estimators of $p_{1}(\mathbf{x})=p(\mathbf{x}, 1)$ and $p_{0}(\mathbf{x})=p(\mathbf{x}, 0)$ defined in (5.4). To study the performance of $\widetilde{\psi}_{n}$ we first state the following result. In what follows $L_{n}\left(\widetilde{\psi}_{n}\right)=P\left\{\widetilde{\psi}_{n}(\mathbf{Z}, \delta) \neq Y \mid \mathcal{D}_{n}\right\}$.

Theorem 5.2.2 Let $\mathcal{M}$ be a totally bounded class of functions $\eta: \mathcal{R}^{p} \rightarrow[0,1]$, containing $\eta_{B}$. Then for every $\epsilon>0$ and all $n \geqslant 1$

$$
P\left\{L_{n}\left(\widetilde{\psi}_{n}\right)-L\left(\psi_{B}\right)>\epsilon\right\} \leqslant 2 \mathcal{N}_{\infty}\left(\frac{\epsilon^{2}}{2048}, \mathcal{M}\right) e^{-C^{\prime} n \epsilon^{4}}+\delta_{n}\left(\frac{\epsilon}{8}\right),
$$

where $C^{\prime}$ is positive constant not depending on $n$ or $\epsilon$ and

$$
\begin{aligned}
\delta_{n}(\cdot) & =P\left\{E\left[\left|\lambda_{n}(\mathbf{X})-\lambda_{B}(\mathbf{X})\right| \mid \mathcal{D}_{n}\right]>\cdot\right\} \\
& +P\left\{E\left[\left|\widehat{p}_{1}(\mathbf{X})-p_{1}(\mathbf{X})\right| \mid \mathcal{D}_{n}\right]>\cdot\right\}+P\left\{E\left[\left|\widehat{p}_{0}(\mathbf{X})-p_{0}(\mathbf{X})\right| \mid \mathcal{D}_{n}\right]>\cdot\right\}
\end{aligned}
$$

The following result is a simple corollary to Theorem 5.2.2.

\section{Corollary 5.2.3 If}

$$
\sum_{n=1}^{\infty} \delta_{n}\left(\frac{\epsilon}{8}\right)<\infty
$$

then an application of the Borel-Cantelli lemma gives

$$
L_{n}\left(\widetilde{\psi}_{n}\right) \stackrel{a . s .}{\rightarrow} L\left(\psi_{B}\right) \quad \text { as } n \rightarrow \infty
$$

For the definitions of totally boundedness and $\mathcal{N}_{\infty}(\epsilon, \mathcal{M})$, see Definitions 1.4.1 and 1.4.2 respectively. It is worth noting that, many important classes of functions are totally bounded with respect to $\|\cdot\|_{\infty}$; here we give two examples: 
Example 1. (Differentiable functions.) For $i=1, \cdots s$, let $k_{i} \geqslant 0$ be non-negative integers. Put $\mathbf{k}=\left(k_{1}, \cdots k_{s}\right)$ and let $k=k_{1}+\cdots+k_{s}$. Also, for any $g: \mathcal{R}^{s} \rightarrow$ $\mathcal{R}$, define $D^{(\mathbf{k})} g(\mathbf{u})=\partial^{k} g(\mathbf{u}) / \partial u_{1}^{k_{1}}, \cdots, \partial u_{s}^{k_{s}}$. Consider the class of functions with bounded partial derivatives of order $r$ :

$$
\mathcal{G}=\left\{g:[0,1]^{p} \rightarrow \mathcal{R}^{1}\left|\sum_{k \leqslant r} \sup _{\mathbf{u}}\right| D^{(\mathbf{k})} g(\mathbf{u}) \mid \leqslant A<\infty\right\} .
$$

Then, for every $\epsilon>0, \log \mathcal{N}_{\infty}(\epsilon, \mathcal{G}) \leqslant M \epsilon^{-\alpha}$, where $\alpha=p / r$ and $M \equiv M(p, r)$; this is due to Kolmogorov and Tikhomirov (1959).

Example 2. Consider the class $\Psi$ of all convex functions $\psi: \mathcal{C} \rightarrow[0,1]$, where $\mathcal{C} \subset \mathcal{R}^{p}$ is compact and convex. If $\psi$ satisfies $\left|\psi\left(\mathbf{z}_{1}\right)-\psi\left(\mathbf{z}_{2}\right)\right| \leqslant L\left|\mathbf{z}_{1}-\mathbf{z}_{2}\right|$, for all $\mathbf{z}_{1}, \mathbf{z}_{2} \in \mathcal{C}$, then $\log \mathcal{N}_{\infty}(\epsilon, \Psi) \leqslant M \epsilon^{-p / 2}$, for every $\epsilon>0$, where $M \equiv M(p, L)$; see van der Vaart and Wellner (1996).

Remark 5.2.4 Finding a bound on $P\left\{E\left[\left|\lambda_{n}(\mathbf{X})-\lambda_{B}(\mathbf{X})\right| \mid \mathcal{D}_{n}\right]>\epsilon\right\}$ clearly depends on the estimator $\lambda_{n}$. For example, if $\lambda_{n}=\underset{\lambda \in \Lambda}{\operatorname{argmin}} \frac{1}{n} \sum_{i=1}^{n}\left|Y_{i}-\lambda\left(\mathbf{X}_{i}\right)\right|^{2}$ and if $\lambda_{B} \in \Lambda$, then, provided that $\Lambda$ is totally bounded, the results of Devroye et al. (1996) yield the exponential bound:

$$
P\left\{E\left[\left|\lambda_{n}(\mathbf{X})-\lambda_{B}(\mathbf{X})\right| \mid \mathcal{D}_{n}\right]>\epsilon\right\} \leqslant 2 \mathcal{N}_{\infty}\left(\epsilon^{2} / 6, \Lambda\right) e^{-n \epsilon^{4} / 18}
$$

Remark 5.2.5 Finding bounds on $P\left\{E\left[\left|\widehat{p}_{1}(\mathbf{X})-p_{1}(\mathbf{X})\right| \mid \mathcal{D}_{n}\right]>\epsilon\right\} \quad$ and $P\left\{E\left[\left|\widehat{p}_{0}(\mathbf{X})-p_{0}(\mathbf{X})\right| \mid \mathcal{D}_{n}\right]>\epsilon\right\}$ depend on the estimators $\widehat{p}_{1}(\mathbf{x})$ and $\widehat{p}_{0}(\mathbf{x})$. For example, for kernel regression estimates,

$$
\widehat{p}_{k}(\mathbf{x})=\sum_{i: Y_{i}=k} \delta_{i} \mathcal{J}\left(\frac{\mathbf{X}_{i}-\mathbf{x}}{h_{n}}\right) / \sum_{i: Y_{i}=k} \mathcal{J}\left(\frac{\mathbf{X}_{i}-\mathbf{x}}{h_{n}}\right),
$$


where $0 / 0$ is defined to be 0 and $\mathcal{J}: \mathcal{R}^{d} \rightarrow \mathcal{R}$ is the kernel used. This gives

$$
\widehat{p}_{1}(\mathbf{x})=\frac{\sum_{i=1}^{n} Y_{i} \delta_{i} \mathcal{J}\left(\frac{\mathbf{x}_{i}-\mathbf{x}}{h_{n}}\right)}{\sum_{i=1}^{n} Y_{i} \mathcal{J}\left(\frac{\mathbf{x}_{i}-\mathbf{x}}{h_{n}}\right)}, \quad \widehat{p}_{0}(\mathbf{x})=\frac{\sum_{i=1}^{n}\left(1-Y_{i}\right) \delta_{i} \mathcal{J}\left(\frac{\mathbf{x}_{i}-\mathbf{x}}{h_{n}}\right)}{\sum_{i=1}^{n}\left(1-Y_{i}\right) \mathcal{J}\left(\frac{\mathbf{x}_{i}-\mathbf{x}}{h_{n}}\right)} .
$$

Let $\mathcal{J}: \mathcal{R}^{d} \rightarrow \mathcal{R}$ be a regular kernel (see, Definition 1.3.2). If $h_{n} \rightarrow 0$ and $n h_{n}^{d} \rightarrow \infty$, as $n \rightarrow \infty$, then for every $\epsilon>0$ there is an integer $n_{0}$ such that for $n>n_{0}$

$$
\begin{aligned}
& P\left\{E\left[\left|\widehat{p}_{1}(\mathbf{X})-p_{1}(\mathbf{X})\right| \mid \mathcal{D}_{n}\right]>\epsilon\right\}+P\left\{E\left[\left|\widehat{p}_{0}(\mathbf{X})-p_{0}(\mathbf{X})\right| \mid \mathcal{D}_{n}\right]>\epsilon\right\} \\
& \leqslant 4 e^{-\lambda_{m i n}^{2} n \epsilon^{2} / 32 \rho_{1}^{2}}
\end{aligned}
$$

where $\lambda_{\min }=\inf _{\mathbf{x}} E(Y \mid \mathbf{X}=\mathbf{x})$ and $\rho_{1}$ is an constant not depending on $n$. We have used the fact that

$$
\begin{aligned}
& P\left\{E\left[\left|\widehat{p}_{1}(\mathbf{X})-p_{1}(\mathbf{X})\right| \mid \mathcal{D}_{n}\right]>\epsilon\right\} \\
& =P\left\{E\left[\left|\frac{\sum_{i=1}^{n} Y_{i} \delta_{i} \mathcal{J}\left(\frac{\mathbf{x}_{i}-\mathbf{x}}{h_{n}}\right)}{\sum_{i=1}^{n} Y_{i} \mathcal{J}\left(\frac{\mathbf{X}_{i}-\mathbf{x}}{h_{n}}\right)}-p_{1}(\mathbf{x})\right| \mid \mathcal{D}_{n}\right]>\epsilon\right\} \\
& =P\left\{E\left[\left|\frac{\sum_{i=1}^{n} Y_{i} \delta_{i} \mathcal{J}\left(\frac{\mathbf{X}_{i}-\mathbf{x}}{h_{n}}\right) / E \mathcal{J}\left(\frac{\mathbf{X}-\mathbf{x}}{h_{n}}\right)}{\sum_{i=1}^{n} Y_{i} \mathcal{J}\left(\frac{\mathbf{X}_{i}-\mathbf{x}}{h_{n}}\right) / E \mathcal{J}\left(\frac{\mathbf{X}-\mathbf{x}}{h_{n}}\right)}-\frac{E(\delta Y \mid \mathbf{X})}{E(Y \mid \mathbf{X})}\right| \mid \mathcal{D}_{n}\right]>\epsilon\right\}
\end{aligned}
$$

by the MAR assumption

$$
\begin{aligned}
& \leqslant P\left\{E\left[\left|\frac{\sum_{i=1}^{n} Y_{i} \delta_{i} \mathcal{J}\left(\frac{\mathbf{X}_{i}-\mathbf{x}}{h_{n}}\right)}{E \mathcal{J}\left(\frac{\mathbf{X}-\mathbf{x}}{h_{n}}\right)}-E(\delta Y \mid \mathbf{X})\right| \mid \mathcal{D}_{n}\right]>\frac{\lambda_{\min } \epsilon}{2}\right\} \\
& +P\left\{E\left[\left|\frac{\sum_{i=1}^{n} Y_{i} \mathcal{J}\left(\frac{\mathbf{X}_{i}-\mathbf{x}}{h_{n}}\right)}{E \mathcal{J}\left(\frac{\mathbf{X}-\mathbf{x}}{h_{n}}\right)}-E(Y \mid \mathbf{X})\right| \mid \mathcal{D}_{n}\right]>\frac{\lambda_{\min } \epsilon}{2}\right\}
\end{aligned}
$$

$\leqslant 2 e^{-n \lambda_{m i n}^{2} \epsilon^{2} / 32 \rho_{1}^{2}} . \quad$ (via the results of Devroye and Krzyzak (1989))

$A$ similar argument is used to bound $P\left\{E\left[\left|\widehat{p}_{0}(\mathbf{X})-p_{0}(\mathbf{X})\right| \mid \mathcal{D}_{n}\right]>\epsilon\right\}$. 


\section{Proof of Theorem 5.2.2.}

First note that from the Cauchy-Schwarz inequality

$$
E\left[\left|\widetilde{\eta}_{n}(\mathbf{Z})-\eta_{B}(\mathbf{Z})\right| \mid \mathcal{D}_{n}\right] \leqslant \sqrt{E\left[\left|\widetilde{\eta}_{n}(\mathbf{Z})-\eta_{B}(\mathbf{Z})\right|^{2} \mid \mathcal{D}_{n}\right]}
$$

On the other hand

$$
\begin{aligned}
E\left[\left|\widetilde{\eta}_{n}(\mathbf{Z})-Y\right|^{2} \mid \mathcal{D}_{n}\right]= & \left.\left.E\left[\mid \widetilde{\eta}_{n}(\mathbf{Z})-\eta_{B}(\mathbf{Z})+\eta_{B}(\mathbf{Z})-Y\right)\right|^{2} \mid \mathcal{D}_{n}\right] \\
= & E\left[\left(\widetilde{\eta}_{n}(\mathbf{Z})-\eta_{B}(\mathbf{Z})\right)^{2} \mid \mathcal{D}_{n}\right]+E\left[\left(\eta_{B}(\mathbf{Z})-Y\right)^{2}\right] \\
& +2 E\left[\left(\widetilde{\eta}_{n}(\mathbf{Z})-\eta_{B}(\mathbf{Z})\right)\left(\eta_{B}(\mathbf{Z})-Y\right) \mid \mathcal{D}_{n}\right] \\
& =E\left[\left(\widetilde{\eta}_{n}(\mathbf{Z})-\eta_{B}(\mathbf{Z})\right)^{2} \mid \mathcal{D}_{n}\right]+E\left[\left(\eta_{B}(\mathbf{Z})-Y\right)^{2}\right]
\end{aligned}
$$

Then

$$
\begin{aligned}
\sqrt{E\left[\left|\widetilde{\eta}_{n}(\mathbf{Z})-\eta_{B}(\mathbf{Z})\right|^{2} \mid \mathcal{D}_{n}\right]} & =\sqrt{E\left[\left|\widetilde{\eta}_{n}(\mathbf{Z})-Y\right|^{2} \mid \mathcal{D}_{n}\right]-E\left|\eta_{B}(\mathbf{Z})-Y\right|^{2}} \\
& =\sqrt{E\left[\left|\widetilde{\eta}_{n}(\mathbf{Z})-Y\right|^{2} \mid \mathcal{D}_{n}\right]-\inf _{\eta \in \mathcal{M}} E|\eta(\mathbf{Z})-Y|^{2}} \\
& :=\sqrt{I(n)}, \quad \text { (say) }
\end{aligned}
$$

But

$$
\begin{aligned}
I(n)= & \sup _{\eta \in \mathcal{M}}\left[E\left[\left|\widetilde{\eta}_{n}(\mathbf{Z})-Y\right|^{2} \mid \mathcal{D}_{n}\right]-\widetilde{S}_{n}\left(\widetilde{\eta}_{n}\right)\right. \\
& \left.+\widetilde{S}_{n}\left(\widetilde{\eta}_{n}\right)-\widetilde{S}_{n}(\eta)+\widetilde{S}_{n}(\eta)-E|\eta(\mathbf{Z})-Y|^{2}\right] \\
\leqslant & 2 \sup _{\eta \in \mathcal{M}}\left|\widetilde{S}_{n}(\eta)-E\right| \eta(\mathbf{Z})-\left.Y\right|^{2} \mid .
\end{aligned}
$$

The inequality in $(5.10)$ follows from the fact that $\widetilde{S}_{n}\left(\widetilde{\eta}_{n}\right)-\widetilde{S}_{n}(\eta) \leqslant 0$, as $\widetilde{\eta}_{n}=$ $\underset{\eta \in \mathcal{M}}{\operatorname{argmin}} \widetilde{S}_{n}(\eta)$. Now, by Corollary 2.3 .1 and the arguments leading to (2.19), for 
every $\epsilon>0$,

$$
\begin{aligned}
P\left\{L_{n}\left(\widetilde{\psi}_{n}\right)-L\left(\psi_{B}\right)>\epsilon\right\} \leqslant & P\left\{\sup _{\eta \in \mathcal{M}}\left|\widetilde{S}_{n}(\eta)-E\right| Y-\left.\eta(\mathbf{Z})\right|^{2} \mid>\frac{\epsilon^{2}}{128}\right\} \\
+ & P\left\{E\left[\left|\lambda_{n}(\mathbf{X})-\lambda_{B}(\mathbf{X})\right| \mid \mathcal{D}_{n}\right]>\frac{\epsilon}{8}\right\} \\
& +P\left\{E\left[\left|\widehat{p}_{1}(\mathbf{X})-p_{1}(\mathbf{X})\right| \mid \mathcal{D}_{n}\right]>\frac{\epsilon}{8}\right\} \\
& +P\left\{E\left[\left|\widehat{p}_{0}(\mathbf{X})-p_{0}(\mathbf{X})\right| \mid \mathcal{D}_{n}\right]>\frac{\epsilon}{8}\right\} \\
\leqslant & P\left\{\sup _{\eta \in \mathcal{M}}\left|\widetilde{S}_{n}(\eta)-E\right| Y-\left.\eta(\mathbf{Z})\right|^{2} \mid>\frac{\epsilon^{2}}{128}\right\}+\delta_{n}\left(\frac{\epsilon}{8}\right) .
\end{aligned}
$$

Let $\mathcal{M}_{\frac{\epsilon^{2}}{2048}}$ be a minimal $\frac{\epsilon^{2}}{2048}$-cover of $\mathcal{M}$. That is, for every $\eta \in \mathcal{M}$, there is a $\eta^{*} \in \mathcal{M}_{\frac{\epsilon^{2}}{2048}}$ such that $\left\|\eta-\eta^{*}\right\|_{\infty}<\frac{\epsilon^{2}}{2048}$. Let $g(\mathbf{Z}, Y)=|Y-\eta(\mathbf{Z})|^{2}$ and $g^{*}(\mathbf{Z}, Y)=$ $\left|Y-\eta^{*}(\mathbf{Z})\right|^{2}$ and observe that

$$
\begin{aligned}
\left|\widetilde{S}_{n}(\eta)-E\right| Y-\left.\eta(\mathbf{Z})\right|^{2}\left|\leqslant \frac{1}{n}\right| \sum_{i=1}^{n} \delta_{i} g\left(\mathbf{Z}_{i}, Y_{i}\right)-\sum_{i=1}^{n} \delta_{i} g^{*}\left(\mathbf{Z}_{i}, Y_{i}\right) \mid & \\
& +\frac{1}{n}\left|\sum_{i=1}^{n}\left(1-\delta_{i}\right) E\left[g\left(\mathbf{Z}_{i}, Y_{i}\right)-g^{*}\left(\mathbf{Z}_{i}, Y_{i}\right) \mid \mathbf{X}_{i}, Y_{i}\right]\right| \\
& +\left|E g(\mathbf{Z}, Y)-E g^{*}(\mathbf{Z}, Y)\right|+\left|\widetilde{S}_{n}\left(\eta^{*}\right)-E\right| Y-\left.\eta^{*}(\mathbf{Z})\right|^{2} \mid \\
\leqslant & \left\|g-g^{*}\right\|_{\infty} \sum_{i=1}^{n} \frac{\delta_{i}}{n}+\left\|g-g^{*}\right\|_{\infty} \sum_{i=1}^{n} \frac{1-\delta_{i}}{n} \\
& +\frac{4 \epsilon^{2}}{2048}+\left|\widetilde{S}_{n}\left(\eta^{*}\right)-E\right| Y-\left.\eta^{*}(\mathbf{Z})\right|^{2} \mid \\
= & \frac{8 \epsilon^{2}}{2048}+\left|\widetilde{S}_{n}\left(\eta^{*}\right)-E\right| Y-\left.\eta^{*}(\mathbf{Z})\right|^{2} \mid .
\end{aligned}
$$

Here, we have used the fact that $\left|g(\mathbf{Z}, Y)-g^{*}(\mathbf{Z}, Y)\right|=|| Y-\left.\eta(\mathbf{Z})\right|^{2}-\mid Y-$ $\left.\eta^{*}(\mathbf{Z})\right|^{2}|=| \eta(\mathbf{Z})-\eta^{*}(\mathbf{Z})|\times| \eta(\mathbf{Z})+\eta^{*}(\mathbf{Z})-2 Y \mid \leqslant 4\left\|\eta-\eta^{*}\right\|_{\infty}$. Therefore, 


$$
\begin{aligned}
& P\left\{\sup _{\eta \in \mathcal{M}}\left|\widetilde{S}_{n}(\eta)-E\right| Y-\left.\eta(\mathbf{Z})\right|^{2} \mid>\frac{\epsilon^{2}}{128}\right\} \\
& \quad \leqslant P\left\{\sup _{\eta \in \mathcal{M}}\left|\widetilde{S}_{\frac{\epsilon^{2}}{2048}}(\eta)-E\right| Y-\left.\eta(\mathbf{Z})\right|^{2} \mid>\frac{\epsilon^{2}}{256}\right\} \\
& \quad \leqslant \mathcal{N}_{\infty}\left(\frac{\epsilon^{2}}{2048}, \mathcal{M}\right) \sup _{\eta \in \mathcal{M}} P\left\{\left|\widetilde{S}_{\frac{\epsilon^{2}}{2048}}(\eta)-E\right| Y-\left.\eta(\mathbf{Z})\right|^{2}>\frac{\epsilon^{2}}{256}\right\} .
\end{aligned}
$$

The Theorem now follows from an application of Hoeffding's inequality to the above probability on the far right hand side. (Note that $\widetilde{S}_{n}(\eta)$ is a sum of i.i.d. bounded random variables, and is also unbiased for $E|Y-\eta(\mathbf{Z})|$ by lemma 5.2.1.)

Remark 5.2.6 Since in the above probability (5.11), the supremum is taken over a possibly uncountable set, there may be some measurability problems. In the book of van der Vaart and Wellner (1996) this issue is handled very elegantly using the notion of outer probability. Alternatively, Dudley (1978) mentioned the universal separability assumption as a way of avoiding measurability difficulties. That is, a class $\mathcal{M}$ of functions is universally separable if there exists a countable subclass $\mathcal{M}_{0}$ such that each $\eta \in \mathcal{M}$ can be written as a pointwise limit of a sequence in $\mathcal{M}_{0}$. Here and in the sequel, we shall assume that the supremum functionals do satisfy measurability conditions.

\subsubsection{Kernel-Based Imputation}

Clearly the function $E\left[\left|Y_{i}-\eta\left(\mathbf{Z}_{i}\right)\right|^{2} \mid \mathbf{X}_{i}, Y_{i}\right]$ that appears in the definition of $\widetilde{S}_{n}(\eta)$ is virtually always unknown in practice. In this section we propose a kernel-based 
estimator of the above conditional expectation. Kernel estimation/imputation has already been used by many other authors; see, for example, Rao and Wang (2002) for empirical likelihood-based inference under kernel regression imputation for missing response data. Also, see Cheng and Chu (1996) for the estimation of a marginal distribution function and Hazelton's (2000) estimator of a marginal density function as well as Cheng's (1994) estimator of a mean functional, in the presence of missing data. To this end, for each $\eta \in \mathcal{M}$, define

$$
\widehat{L}_{n}(\eta)=\frac{1}{n} \sum_{i=1}^{n}\left[\delta_{i}\left|Y_{i}-\eta\left(\mathbf{Z}_{i}\right)\right|^{2}+\left(1-\delta_{i}\right) \widehat{\ell}_{n, i}(\eta)\right],
$$

where the term $\widehat{\ell}_{n, i}$, defined by

$$
\widehat{\ell}_{n, i}(\eta)=\frac{\sum_{j=1, \neq i}^{n} \delta_{j} I\left\{Y_{j}=Y_{i}\right\} \mathcal{H}\left(\frac{\mathbf{x}_{j}-\mathbf{x}_{i}}{h_{n, \mathbf{x}}}\right)\left|Y_{j}-\eta\left(\mathbf{Z}_{j}\right)\right|^{2}}{\sum_{j=1, \neq i}^{n} \delta_{j} I\left\{Y_{j}=Y_{i}\right\} \mathcal{H}\left(\frac{\mathbf{x}_{j}-\mathbf{x}_{i}}{h_{n, \mathbf{x}}}\right)}
$$

is the estimate of the function $E\left[\left|Y_{i}-\eta\left(\mathbf{Z}_{i}\right)\right|^{2} \mid \mathbf{X}_{i}, Y_{i}\right]$. Note that $\widehat{L}_{n}(\eta)$ is the imputation estimator of $E\left|Y_{i}-\eta\left(\mathbf{Z}_{i}\right)\right|^{2}$. Also, let

$$
\widehat{\eta}_{n}=\underset{\eta \in \mathcal{M}}{\operatorname{argmin}} \widehat{L}_{n}(\eta)
$$

be the resulting empirically selected function from the class $\mathcal{M}$. Define the classifier corresponding to $\widehat{\eta}_{n}$ by

$$
\widehat{\psi}_{n}(\mathbf{Z}, \delta)=\delta \widehat{\psi}_{n, 1}(\mathbf{Z})+(1-\delta) \psi_{n, 0}(\mathbf{X})
$$

where $\widehat{\psi}_{n, 1}(\mathbf{Z})=I\left\{\widehat{p}_{1}(\mathbf{X}) \widehat{\eta}_{n}(\mathbf{Z})-\widehat{p}_{0}(\mathbf{X})\left(1-\widehat{\eta}_{n}(\mathbf{Z})\right)>0\right\}$ and $\psi_{0}(\mathbf{X})=I\{(1-$ $\left.\left.\widehat{p}_{1}(\mathbf{X})\right) \lambda_{n}(\mathbf{X})-\left(1-\widehat{p}_{0}(\mathbf{X})\right)\left(1-\lambda_{n}(\mathbf{X})\right)>0\right\}$; here $\lambda_{n}(\mathbf{x}), \widehat{p}_{1}(\mathbf{x})$, and $\widehat{p}_{0}(\mathbf{x})$ are any estimators of $\lambda(\mathbf{x})=P\{Y=1 \mid \mathbf{X}=\mathbf{x}\}, p_{1}(\mathbf{x})$, and $p_{0}(\mathbf{x})$, respectively. In order to assess the performance of $\widehat{\psi}_{n}$, we state some conditions. Our first condition assumes that the conditional probability that $\delta=1$ is always nonzero, i.e., 
C1. $\quad p_{\min }:=\inf _{\mathbf{x}} P\{\delta=1 \mid \mathbf{X}=\mathbf{x}, Y=y\}>0, \quad y=0,1$.

Also, in order to avoid having unstable estimates (in the tails of the pdf $f$ of $\mathbf{X}$ ), it is often assumed in the literature on nonparametric regression that $f$ is compactly supported and is bounded away from zero, and so we shall make this assumption. More specifically, it is assumed that

C2. The probability density function of $\mathbf{X}$, say $f(\mathbf{x})$ is compactly supported and satisfies: $\inf _{\mathbf{x}} f(\mathbf{x}):=f_{m i n}>0$. Furthermore both $f$ and its $1^{\text {st }}$-order partial derivatives are uniformly bounded on this compact support.

As for the choice of kernel, we shall require

C3. The kernel $\mathcal{H}$ is a pdf and satisfies $\int\left|t_{i}\right| \mathcal{H}(\mathbf{t}) d \mathbf{t}<\infty, i=1, \cdots, d$ and $\|\mathcal{H}\|_{\infty}<\infty$. Furthermore, the smoothing parameter $h_{n, \mathbf{x}}$ satisfies $h_{n, \mathbf{x}} \rightarrow 0$ and $n h_{n, \mathbf{x}}^{d} \rightarrow \infty$, as $n \rightarrow \infty$.

C4. For every $\eta \in \mathcal{M}$, the partial derivatives $\frac{\partial}{\partial x_{i}}\left\{E\left[\delta|Y-\eta(\mathbf{Z})|^{2} \mid \mathbf{X}=\mathbf{x}, Y=y\right]\right\}$, (where $i=1, \cdots, d$, and $y=0$ or 1 ) exist and are bounded on the compact support of $f$, uniformly in $\mathbf{x}$.

Conditions C1 to C3 are standard in literature. Condition C4 is technical. The following theorem provides bounds on the performance of $\widehat{\psi}_{n}$.

Theorem 5.2.7 Let $\mathcal{M}$ be the class of functions defined in Theorem 5.2.2, and suppose that conditions $\mathbf{C 1 - C 4}$ hold. Then, for every $\epsilon>0$, there is an $n_{0}>0$ such that for all $n \geqslant n_{0}$,

$$
P\left\{L_{n}\left(\widehat{\psi}_{n}\right)-L\left(\psi_{B}\right)>\epsilon\right\} \leqslant C_{1} n \mathcal{N}_{\infty}\left(\epsilon^{2} / 2048, \mathcal{M}\right) e^{-C_{2} n h_{n, \mathbf{x}}^{d} \epsilon^{4}}+\delta_{n}\left(\frac{\epsilon}{8}\right)
$$

where $C_{1}$ and $C_{2}$ are positive constants not depending on $n$ and $\delta_{n}(\cdot)$ is defined in (5.7). 


\section{Proof.}

Define the class of functions

$$
\mathcal{G} \equiv \mathcal{G}_{\mathcal{M}}=\left\{g: \mathcal{R}^{p+1} \rightarrow[0,1]|g(\mathbf{Z}, Y)=| Y-\left.\eta(\mathbf{Z})\right|^{2}, \exists \eta \in \mathcal{M}\right\}
$$

Also, let $\mu(g)=E g(\mathbf{Z}, Y)=E|Y-\eta(\mathbf{Z})|^{2}$ and put

$$
\mu_{n}(g)=n^{-1} \sum_{i=1}^{n}\left[\delta_{i} g\left(\mathbf{Z}_{i}, Y_{i}\right)+\left(1-\delta_{i}\right){\left.\widehat{\left(\mathbf{Z}_{i}, Y_{i}\right.}\right)}\right]
$$

where

$$
\left.\widehat{g\left(\mathbf{Z}_{i}, Y_{i}\right.}\right)=\frac{\sum_{j=1, \neq i}^{n} \delta_{j} I\left\{Y_{j}=Y_{i}\right\} \mathcal{H}\left(\frac{\mathbf{x}_{j}-\mathbf{x}_{i}}{h_{n, \mathbf{x}}}\right) g\left(\mathbf{Z}_{j}, Y_{j}\right)}{\sum_{j=1, \neq i}^{n} \delta_{j} I\left\{Y_{j}=Y_{i}\right\} \mathcal{H}\left(\frac{\mathbf{x}_{j}-\mathbf{x}_{i}}{h_{n, \mathbf{x}}}\right)}
$$

By Corollary 2.3.1 and arguments similar to those leading to (5.9) and (5.10) one finds

$$
\begin{aligned}
L_{n}\left(\widehat{\psi}_{n}\right)-L\left(\psi_{B}\right) \leqslant & 2 \sqrt{2 \sup _{g \in \mathcal{G}}\left|\mu_{n}(g)-\mu(g)\right|}+2 E\left[\left|\lambda_{n}(\mathbf{X})-\lambda(\mathbf{X})\right| \mid \mathcal{D}_{n}\right] \\
& +2 E\left[\left|\widehat{p}_{1}(\mathbf{X})-p_{1}(\mathbf{X})\right| \mid \mathcal{D}_{n}\right]+2 E\left[\left|\widehat{p}_{0}(\mathbf{X})-p_{0}(\mathbf{X})\right| \mid \mathcal{D}_{n}\right]
\end{aligned}
$$

Therefore, $\forall \epsilon>0$,

$$
P\left\{L_{n}\left(\widehat{\psi}_{n}\right)-L\left(\psi_{B}\right)>\epsilon\right\} \leqslant P\left\{\sup _{g \in \mathcal{G}}\left|\mu_{n}(g)-\mu(g)\right|>\frac{\epsilon^{2}}{128}\right\}+\delta_{n}\left(\frac{\epsilon}{8}\right) .
$$

But

$$
\begin{aligned}
P\left\{\sup _{g \in \mathcal{G}}\left|\mu_{n}(g)-\mu(g)\right|>\frac{\epsilon^{2}}{128}\right\} & \leqslant P\left\{\sup _{\left.g \in \mathcal{G}_{\epsilon^{2} / 2048}\left|\mu_{n}(g)-\mu(g)\right|>\frac{\epsilon^{2}}{256}\right\}}\right. \\
& \left(\text { where } \mathcal{G}_{\epsilon^{2} / 2048} \text { is an } \frac{\epsilon^{2}}{2048}-\text { cover of } \mathcal{G} \text { w.r.t. }\|\|_{\infty}\right) \\
& \leqslant \mathcal{N}_{\infty}\left(\frac{\epsilon^{2}}{2048}, \mathcal{M}\right)_{g \in \mathcal{G}_{\epsilon^{2} / 2048}} P\left\{\left|\mu_{n}(g)-\mu(g)\right|>\frac{\epsilon^{2}}{256}\right\} .
\end{aligned}
$$


To bound the probability on the far right side of (5.15), first observe that for every $t>0$, one may write

$$
\begin{aligned}
& P\left\{\left|\mu_{n}(g)-\mu(g)\right|>t\right\} \\
& \leqslant P\left\{n^{-1}\left|\sum_{i=1}^{n} g\left(\mathbf{Z}_{i}, Y_{i}\right)-n \mu(g)\right|>\frac{t}{3}\right\} \\
+ & P\left\{n^{-1}\left|\sum_{i=1}^{n}\left(1-\delta_{i}\right)\left(g\left(\mathbf{Z}_{i}, Y_{i}\right)-E\left[g\left(\mathbf{Z}_{i}, Y_{i}\right) \mid \mathbf{X}_{i}, Y_{i}\right]\right)\right|>\frac{t}{3}\right\} \\
+ & P\left\{n^{-1}\left|\sum_{i=1}^{n}\left(1-\delta_{i}\right)\left(\sum_{j=1, \neq i}^{n} \delta_{j} W_{j}\left(\mathbf{X}_{i}, Y_{i}\right) g\left(\mathbf{Z}_{j}, Y_{j}\right)-E\left[g\left(\mathbf{Z}_{i}, Y_{i}\right) \mid \mathbf{X}_{i}, Y_{i}\right]\right)\right|>\frac{t}{3}\right\} \\
:= & D_{n 1}+D_{n 2}+D_{n 3},
\end{aligned}
$$

where

$$
W_{j}\left(\mathbf{X}_{i}, Y_{i}\right)=\frac{I\left\{Y_{j}=Y_{i}\right\} \mathcal{H}\left(\frac{\mathbf{x}_{j}-\mathbf{x}_{i}}{h_{n, \mathbf{x}}}\right)}{\sum_{k=1, \neq i}^{n} \delta_{k} I\left\{Y_{k}=Y_{i}\right\} \mathcal{H}\left(\frac{\mathbf{X}_{k}-\mathbf{X}_{i}}{h_{n, \mathbf{x}}}\right)} .
$$

But by Hoeffding's inequality $D_{n 1} \leqslant 2 e^{-\frac{n t^{2}}{18}}$. Similarly, the terms

$$
U_{i}=\left(1-\delta_{i}\right)\left(g\left(\mathbf{Z}_{i}, Y_{i}\right)-E\left[g\left(\mathbf{Z}_{i}, Y_{i}\right) \mid \mathbf{X}_{i}, Y_{i}\right]\right), \quad i=1, \cdots, n
$$

are independent, zero-mean (under the MAR assumption) random variables, bounded by -1 and +1 . The fact that $E U_{i}=0$ follows from:

$$
\begin{aligned}
E U_{i} & =E\left\{E\left[\left(1-\delta_{i}\right)\left(g\left(\mathbf{Z}_{i}, Y_{i}\right)-E\left[g\left(\mathbf{Z}_{i}, Y_{i}\right) \mid \mathbf{X}_{i}, Y_{i}\right]\right) \mid \mathbf{Z}_{i}, Y_{i}\right]\right\} \\
& =E\left\{\left(g\left(\mathbf{Z}_{i}, Y_{i}\right)-E\left[g\left(\mathbf{Z}_{i}, Y_{i}\right) \mid \mathbf{X}_{i}, Y_{i}\right]\right) E\left[\left(1-\delta_{i}\right) \mid \mathbf{Z}_{i}, Y_{i}\right]\right\} \\
& \stackrel{\text { by }}{=} E\left\{E\left[\left(1-p\left(\mathbf{X}_{i}, Y_{i}\right)\right)\left(g\left(\mathbf{Z}_{i}, Y_{i}\right)-E\left[g\left(\mathbf{Z}_{i}, Y_{i}\right) \mid \mathbf{X}_{i}, Y_{i}\right]\right) \mid \mathbf{X}_{i}, Y_{i}\right]\right\}=0 .
\end{aligned}
$$

Thus, one more application of Hoeffding's inequality gives

$$
D_{n 2}=P\left\{n^{-1}\left|\sum_{i=1}^{n} U_{i}\right|>\frac{t}{3}\right\} \leqslant 2 e^{-\frac{n t^{2}}{18}} .
$$

106 
As for the term $D_{n 3}$, first note that by the MAR assumption $E\left[g\left(\mathbf{Z}_{i}, Y_{i}\right) \mid \mathbf{X}_{i}, Y_{i}\right]=$ $\left(p\left(\mathbf{X}_{i}, Y_{i}\right)\right)^{-1} \times E\left[\delta_{i} g\left(\mathbf{Z}_{i}, Y_{i}\right) \mid \mathbf{X}_{i}, Y_{i}\right]$, where $p\left(\mathbf{X}_{i}, Y_{i}\right)=E\left\{\delta_{i} \mid \mathbf{X}_{i}, Y_{i}\right\}$ is as defined in (5.5). Thus

$$
\begin{aligned}
& D_{n 3} \leqslant P\left\{n^{-1} \sum_{i=1}^{n}\left|\sum_{j=1, \neq i}^{n} \delta_{j} W_{j}\left(\mathbf{X}_{i}, Y_{i}\right) g\left(\mathbf{Z}_{j}, Y_{j}\right)-E\left[g\left(\mathbf{Z}_{i}, Y_{i}\right) \mid \mathbf{X}_{i}, Y_{i}\right]\right|>\frac{t}{3}\right\} \\
& \leqslant \sum_{i=1}^{n} P\left\{\mid \frac{\sum_{j=1, \neq i}^{n} \delta_{j} I\left\{Y_{j}=Y_{i}\right\} \mathcal{H}\left(\frac{\mathbf{x}_{j}-\mathbf{x}_{i}}{h_{n, \mathbf{x}}}\right) g\left(\mathbf{Z}_{j}, Y_{j}\right)}{\sum_{j=1, \neq i}^{n} \delta_{j} I\left\{Y_{j}=Y_{i}\right\} \mathcal{H}\left(\frac{\mathbf{x}_{j}-\mathbf{X}_{i}}{h_{n, \mathbf{x}}}\right)}\right. \\
& \left.-\frac{E\left[\delta_{i} g\left(\mathbf{Z}_{i}, Y_{i}\right) \mid \mathbf{X}_{i}, Y_{i}\right]}{p\left(\mathbf{X}_{i}, Y_{i}\right)} \mid>\frac{t}{3}\right\} \\
& :=\sum_{i=1}^{n} p_{n, i}, \quad \text { (say) }
\end{aligned}
$$

Let

$$
\begin{aligned}
\mu\left(g \mid \mathbf{X}_{i}, Y_{i}\right) & =E\left[\delta_{i} g\left(\mathbf{Z}_{i}, Y_{i}\right) \mid \mathbf{X}_{i}, Y_{i}\right] \\
\widehat{\mu}\left(g \mid \mathbf{X}_{i}, Y_{i}\right) & =\frac{\sum_{j=1, \neq i}^{n} \delta_{j} I\left\{Y_{j}=Y_{i}\right\} \mathcal{H}\left(\frac{\mathbf{x}_{j}-\mathbf{X}_{i}}{h_{n, \mathbf{x}}}\right) g\left(\mathbf{Z}_{j}, Y_{j}\right)}{\sum_{j=1, \neq i}^{n} I\left\{Y_{j}=Y_{i}\right\} \mathcal{H}\left(\frac{\mathbf{x}_{j}-\mathbf{X}_{i}}{h_{n, \mathbf{x}}}\right)} \\
\widehat{p}\left(\mathbf{X}_{i}, Y_{i}\right) & =\frac{\sum_{j=1, \neq i}^{n} \delta_{j} I\left\{Y_{j}=Y_{i}\right\} \mathcal{H}\left(\frac{\mathbf{x}_{j}-\mathbf{X}_{i}}{h_{n, \mathbf{x}}}\right)}{\sum_{j=1, \neq i}^{n} I\left\{Y_{j}=Y_{i}\right\} \mathcal{H}\left(\frac{\mathbf{x}_{j}-\mathbf{X}_{i}}{h_{n, \mathbf{x}}}\right)} .
\end{aligned}
$$

Then, one finds

$$
\begin{aligned}
& p_{n, i}=P\left\{\left|\frac{\widehat{\mu}\left(g \mid \mathbf{X}_{i}, Y_{i}\right)}{\widehat{p}\left(\mathbf{X}_{i}, Y_{i}\right)}-\frac{\mu\left(g \mid \mathbf{X}_{i}, Y_{i}\right)}{p\left(\mathbf{X}_{i}, Y_{i}\right)}\right|>\frac{t}{3}\right\} \\
& =P\left\{\mid \frac{-\widehat{\mu}\left(g \mid \mathbf{X}_{i}, Y_{i}\right) / \widehat{p}\left(\mathbf{X}_{i}, Y_{i}\right)}{p\left(\mathbf{X}_{i}, Y_{i}\right)}\left(\widehat{p}\left(\mathbf{X}_{i}, Y_{i}\right)-p\left(\mathbf{X}_{i}, Y_{i}\right)\right)\right. \\
& \left.+\frac{\widehat{\mu}\left(g \mid \mathbf{X}_{i}, Y_{i}\right)-\mu\left(g \mid \mathbf{X}_{i}, Y_{i}\right)}{p\left(\mathbf{X}_{i}, Y_{i}\right)} \mid>\frac{t}{3}\right\} \text {. }
\end{aligned}
$$

Using the fact that $\left|\widehat{\mu}\left(g \mid \mathbf{X}_{i}, Y_{i}\right) / \widehat{p}\left(\mathbf{X}_{i}, Y_{i}\right)\right| \leqslant 1$, we can write

$$
p_{n, i} \leqslant P\left\{\left|\widehat{p}\left(\mathbf{X}_{i}, Y_{i}\right)-p\left(\mathbf{X}_{i}, Y_{i}\right)\right|+\left|\widehat{\mu}\left(g \mid \mathbf{X}_{i}, Y_{i}\right)-\mu\left(g \mid \mathbf{X}_{i}, Y_{i}\right)\right|>\frac{t p_{\min }}{3}\right\} .
$$


Also, define

$$
\begin{aligned}
\widehat{S}\left(g \mid \mathbf{X}_{i}, Y_{i}\right) & =\frac{1}{(n-1) h_{n, \mathbf{x}}^{d}} \sum_{j=1, \neq i}^{n} \delta_{j} I\left\{Y_{j}=Y_{i}\right\} \mathcal{H}\left(\frac{\mathbf{X}_{j}-\mathbf{X}_{i}}{h_{n, \mathbf{x}}}\right) g\left(\mathbf{Z}_{j}, Y_{j}\right) \\
S\left(g \mid \mathbf{X}_{i}, Y_{i}\right) & =f\left(\mathbf{X}_{i}\right) P\left\{Y=Y_{i} \mid Y_{i}\right\} E\left[\delta_{i} g\left(\mathbf{Z}_{i}, Y_{i}\right) \mid \mathbf{X}_{i}, Y_{i}\right] \\
& =f\left(\mathbf{X}_{i}\right) P\left\{Y=Y_{i} \mid Y_{i}\right\} \mu\left(g \mid \mathbf{X}_{i}, Y_{i}\right) \\
\widehat{r}\left(\mathbf{X}_{i}, Y_{i}\right) & =\frac{1}{(n-1) h_{n, \mathbf{x}}^{d}} \sum_{j=1, \neq i}^{n} I\left\{Y_{j}=Y_{i}\right\} \mathcal{H}\left(\frac{\mathbf{X}_{j}-\mathbf{X}_{i}}{h_{n, \mathbf{x}}}\right) \\
r\left(\mathbf{X}_{i}, Y_{i}\right) & =f\left(\mathbf{X}_{i}\right) P\left\{Y=Y_{i} \mid Y_{i}\right\} .
\end{aligned}
$$

Then $\widehat{\mu}\left(g \mid \mathbf{X}_{i}, Y_{i}\right)=\widehat{S}\left(g \mid \mathbf{X}_{i}, Y_{i}\right) / \widehat{r}\left(\mathbf{X}_{i}, Y_{i}\right)$ and $\mu\left(g \mid \mathbf{X}_{i}, Y_{i}\right)=S\left(g \mid \mathbf{X}_{i}, Y_{i}\right) / r\left(\mathbf{X}_{i}, Y_{i}\right)$. Consequently, one finds (with $p_{1}=P(Y=1)$ )

$$
\begin{aligned}
p_{n, i} \leqslant & P\left\{\left|\widehat{p}\left(\mathbf{X}_{i}, Y_{i}\right)-p\left(\mathbf{X}_{i}, Y_{i}\right)\right|+\left|\frac{\widehat{S}\left(g \mid \mathbf{X}_{i}, Y_{i}\right)}{\widehat{r}\left(\mathbf{X}_{i}, Y_{i}\right)}-\frac{S\left(g \mid \mathbf{X}_{i}, Y_{i}\right)}{r\left(\mathbf{X}_{i}, Y_{i}\right)}\right|>\frac{t p_{\min }}{3}\right\} \\
\leqslant & P\left\{\left|\widehat{p}\left(\mathbf{X}_{i}, Y_{i}\right)-p\left(\mathbf{X}_{i}, Y_{i}\right)\right|+\left|\frac{\widehat{r}\left(\mathbf{X}_{i}, Y_{i}\right)-r\left(\mathbf{X}_{i}, Y_{i}\right)}{r\left(\mathbf{X}_{i}, Y_{i}\right)}\right|\right. \\
& \left.+\left|\frac{\widehat{S}\left(g \mid \mathbf{X}_{i}, Y_{i}\right)-S\left(g \mid \mathbf{X}_{i}, Y_{i}\right)}{r\left(\mathbf{X}_{i}, Y_{i}\right)}\right|>\frac{t p_{\min }}{3}\right\} \\
\leqslant & P\left\{\left|\widehat{p}\left(\mathbf{X}_{i}, Y_{i}\right)-p\left(\mathbf{X}_{i}, Y_{i}\right)\right|+\frac{1}{\left(p_{1} \wedge\left(1-p_{1}\right)\right) f_{\min }}\left(\left|\widehat{r}\left(\mathbf{X}_{i}, Y_{i}\right)-r\left(\mathbf{X}_{i}, Y_{i}\right)\right|\right.\right. \\
& \left.\left.+\left|\widehat{S}\left(g \mid \mathbf{X}_{i}, Y_{i}\right)-S\left(g \mid \mathbf{X}_{i}, Y_{i}\right)\right|\right)>\frac{t p_{\min }}{3}\right\},
\end{aligned}
$$

because $r\left(\mathbf{X}_{i}, Y_{i}\right) \geqslant\left(p_{1} \wedge\left(1-p_{1}\right)\right) f_{\min }$. Now fix $\beta>0$ and observe that

$$
\begin{aligned}
P & \left\{\left|\widehat{S}\left(g \mid \mathbf{X}_{i}, Y_{i}\right)-S\left(g \mid \mathbf{X}_{i}, Y_{i}\right)\right|>\beta\right\} \\
& \leqslant E P\left\{\left|\widehat{S}\left(g \mid \mathbf{X}_{i}, Y_{i}\right)-E\left[\widehat{S}\left(g \mid \mathbf{X}_{i}, Y_{i}\right) \mid \mathbf{X}_{i}, Y_{i}\right]\right|\right. \\
& \left.+\left|E\left[\widehat{S}\left(g \mid \mathbf{X}_{i}, Y_{i}\right) \mid \mathbf{X}_{i}, Y_{i}\right]-S\left(g \mid \mathbf{X}_{i}, Y_{i}\right)\right|>\beta \mid \mathbf{X}_{i}, Y_{i}\right\} .
\end{aligned}
$$

To deal with the r.h.s. of the above expression, we need the following lemma (whose proof will be given later) to bound the "bias" term $E\left[\widehat{S}\left(g \mid \mathbf{X}_{i}, Y_{i}\right) \mid \mathbf{X}_{i}, Y_{i}\right]-S\left(g \mid \mathbf{X}_{i}, Y_{i}\right)$. 
Lemma 5.2.8 Suppose that conditions $\mathbf{C 1 - C 4}$ hold. Then

$$
\left|E\left[\widehat{S}\left(g \mid \mathbf{X}_{i}, Y_{i}\right) \mid \mathbf{X}_{i}, Y_{i}\right]-S\left(g \mid \mathbf{X}_{i}, Y_{i}\right)\right| \leqslant h_{n, \mathbf{x}} \cdot \mid \text { const } \mid
$$

As a consequence of Lemma 5.2.8, one finds for every $\beta>0$, and $n$ large enough (such that $h_{n, \mathbf{x}} \cdot \mid$ const $\mid \leqslant \frac{\beta}{2}$ ),

$$
\begin{aligned}
& P\left\{\left|\widehat{S}\left(g \mid \mathbf{X}_{i}, Y_{i}\right)-S\left(g \mid \mathbf{X}_{i}, Y_{i}\right)\right|>\beta\right\} \\
\leqslant & E P\left\{\left|\widehat{S}\left(g \mid \mathbf{X}_{i}, Y_{i}\right)-E\left[\widehat{S}\left(g \mid \mathbf{X}_{i}, Y_{i}\right) \mid \mathbf{X}_{i}, Y_{i}\right]\right|>\frac{\beta}{2} \mid \mathbf{X}_{i}, Y_{i}\right\} \\
= & E P\left\{\frac{1}{n-1}\left|\sum_{j=1, \neq i}^{n} \Gamma_{j}\left(\mathbf{X}_{i}, Y_{i}\right)\right|>\frac{\beta}{2} \mid \mathbf{X}_{i}, Y_{i}\right\}
\end{aligned}
$$

where

$$
\begin{aligned}
\Gamma_{j}\left(\mathbf{X}_{i}, Y_{i}\right)=h_{n, \mathbf{x}}^{-d}\left[\delta_{j} I\left\{Y_{j}=Y_{i}\right\} \mathcal{H}\left(\frac{\mathbf{X}_{j}-\mathbf{X}_{i}}{h_{n, \mathbf{x}}}\right) g\left(\mathbf{Z}_{j}, Y_{j}\right)\right. \\
\left.-E\left\{\delta_{j} I\left\{Y_{j}=Y_{i}\right\} \mathcal{H}\left(\frac{\mathbf{X}_{j}-\mathbf{X}_{i}}{h_{n, \mathbf{x}}}\right) g\left(\mathbf{Z}_{j}, Y_{j}\right) \mid \mathbf{X}_{i}, Y_{i}\right\}\right] .
\end{aligned}
$$

But, conditional on $\left(\mathbf{X}_{i}, Y_{i}\right)$, the terms $\Gamma_{j}$ 's are independent, zero-mean random variables bounded by $-h_{n, \mathbf{x}}^{-d}\|\mathcal{H}\|_{\infty}$ and $+h_{n, \mathbf{x}}^{-d}\|\mathcal{H}\|_{\infty}$. Furthermore, $\operatorname{Var}\left(\Gamma_{j}\left(\mathbf{X}_{i}, Y_{i}\right) \mid \mathbf{X}_{i}, Y_{i}\right)=E\left(\Gamma_{j}^{2}\left(\mathbf{X}_{i}, Y_{i}\right) \mid \mathbf{X}_{i}, Y_{i}\right) \leqslant h_{n, \mathbf{x}}^{-d}\|\mathcal{H}\|_{\infty}\|f\|_{\infty}$. Therefore an application of Bernstein's inequality to the conditional probability on the r.h.s. of (5.19) yields

$$
P\left\{(n-1)^{-1}\left|\sum_{j=1, \neq i}^{n} \Gamma_{j}\left(\mathbf{X}_{i}, Y_{i}\right)\right|>\frac{\beta}{2} \mid \mathbf{X}_{i}, Y_{i}\right\} \leqslant 2 \exp \left\{-\frac{(n-1) h_{n, \mathbf{x}}^{d}(\beta / 2)^{2}}{2\|\mathcal{H}\|_{\infty}\left[\|f\|_{\infty}+\beta / 2\right]}\right\}
$$

Since the above bound does not depend on $\left(\mathbf{X}_{i}, Y_{i}\right)$ one obtains, for $n$ large enough,

$$
P\left\{\left|\widehat{S}\left(g \mid \mathbf{X}_{i}, Y_{i}\right)-S\left(g \mid \mathbf{X}_{i}, Y_{i}\right)\right|>\beta\right\} \leqslant \text { the bound in }(5.20)
$$


Similarly, one can also show (in fact, with less effort ) that for $\forall \beta^{\prime}>0$ and $n$ large enough,

$$
P\left\{\left|\widehat{r}\left(\mathbf{X}_{i}, Y_{i}\right)-r\left(\mathbf{X}_{i}, Y_{i}\right)\right|>\beta^{\prime}\right\} \leqslant 2 e^{-\frac{(n-1) h_{n, \mathbf{x}}^{d}\left(\beta^{\prime} / 2\right)^{2}}{\|\mathcal{H}\| \infty\left[\|f\| \infty+\beta^{\prime} / 2\right]}}
$$

Finally, note that

$$
\begin{aligned}
\left|\widehat{p}\left(\mathbf{X}_{i}, Y_{i}\right)-p\left(\mathbf{X}_{i}, Y_{i}\right)\right| & =\left|\frac{\widehat{S}\left(1 \mid \mathbf{X}_{i}, Y_{i}\right)}{\widehat{r}\left(\mathbf{X}_{i}, Y_{i}\right)}-\frac{S\left(1 \mid \mathbf{X}_{i}, Y_{i}\right)}{r\left(\mathbf{X}_{i}, Y_{i}\right)}\right| \\
& \leqslant\left|\frac{\widehat{r}\left(\mathbf{X}_{i}, Y_{i}\right)-r\left(\mathbf{X}_{i}, Y_{i}\right)}{r\left(\mathbf{X}_{i}, Y_{i}\right)}\right|+\left|\frac{\widehat{S}\left(1 \mid \mathbf{X}_{i}, Y_{i}\right)-S\left(1 \mid \mathbf{X}_{i}, Y_{i}\right)}{r\left(\mathbf{X}_{i}, Y_{i}\right)}\right| .
\end{aligned}
$$

Since $r\left(\mathbf{X}_{i}, Y_{i}\right) \geqslant\left(p_{1} \wedge\left(1-p_{1}\right)\right) f_{\text {min }}$, where $p_{1}=P(Y=1)$, one finds for every $\beta^{\prime \prime}>0$, (and $n$ large enough),

$$
\begin{aligned}
& P\left\{\left|\widehat{p}\left(\mathbf{X}_{i}, Y_{i}\right)-p\left(\mathbf{X}_{i}, Y_{i}\right)\right|>\beta^{\prime \prime}\right\} \\
& \quad \leqslant 4 \exp \left\{\frac{-(n-1) h_{n, \mathbf{x}}^{d}\left(\left(p_{1} \wedge\left(1-p_{1}\right)\right) f_{\min } \beta^{\prime \prime} / 4\right)^{2}}{2\|\mathcal{H}\|_{\infty}\left[\|f\|_{\infty}+\left(\left(p_{1} \wedge\left(1-p_{1}\right)\right) f_{\min } \beta^{\prime \prime} / 4\right)\right]}\right\} .
\end{aligned}
$$

Putting all the above together, one finds, for $n$ large enough,

$$
\begin{aligned}
p_{n, i} \leqslant & P\left\{\left|\widehat{S}\left(g \mid \mathbf{X}_{i}, Y_{i}\right)-S\left(g \mid \mathbf{X}_{i}, Y_{i}\right)\right|>\frac{\left(p_{1} \wedge\left(1-p_{1}\right)\right) f_{\min } p_{\min } t}{9}\right\} \\
& +P\left\{\left|\widehat{r}\left(\mathbf{X}_{i}, Y_{i}\right)-r\left(\mathbf{X}_{i}, Y_{i}\right)\right|>\frac{\left(p_{1} \wedge\left(1-p_{1}\right)\right) f_{\min } p_{\min } t}{9}\right\} \\
& +P\left\{\left|\widehat{p}\left(\mathbf{X}_{i}, Y_{i}\right)-p\left(\mathbf{X}_{i}, Y_{i}\right)\right|>\frac{p_{\min } t}{9}\right\} \\
\leqslant & 2\left[e^{-C_{3}(n-1) h_{n, \mathbf{x}}^{d} t^{2}}+e^{-C_{4}(n-1) h_{n, \mathbf{x}}^{d} t^{2}}+2 e^{-C_{5}(n-1) h_{n, \mathbf{x}}^{d} t^{2}}\right] \\
\leqslant & 8 e^{-C_{6}(n-1) h_{n, \mathbf{x}}^{d} t^{2}},
\end{aligned}
$$

where (5.23) is obtained upon taking $\beta=\beta^{\prime}=\frac{\left(p_{1} \wedge\left(1-p_{1}\right)\right) f_{\min } p_{\min } t}{9}$ and $\beta^{\prime \prime}=\frac{p_{\min } t}{9}$. Here the constants $C_{3}-C_{6}$ are positive and do not depend on $n$. Note that, in general $\exp \left\{-\frac{(n-1) h_{n, c^{\prime}}^{d} t^{2}}{2\|\mathcal{H}\|_{\infty}\left[\|f\|_{\infty}+c^{\prime \prime} t\right]}\right\}$, where $t>0$, is not bounded by $\exp \left\{-c(n-1) h_{n, \mathbf{x}}^{d} t^{2}\right\}$. 
However, in our case, we only need to focus on the case where $0<t<12$ (see below). Now for every $t \in(0,12)$ one has

$$
\begin{aligned}
\exp \left\{-\frac{(n-1) h_{n, \mathbf{x}}^{d} c^{\prime} t^{2}}{2\|\mathcal{H}\|_{\infty}\left[\|f\|_{\infty}+c^{\prime \prime} t\right]}\right\} & \leqslant \exp \left\{-\frac{(n-1) h_{n, \mathbf{x}}^{d} c^{\prime} t^{2}}{2\|\mathcal{H}\|_{\infty}\left[\|f\|_{\infty}+c^{\prime \prime 12}\right]}\right\} \\
& :=\exp \left\{-c(n-1) h_{n, \mathbf{x}} t^{2}\right\} .
\end{aligned}
$$

To see why we only need to consider $t \in(0,12)$, first note that $0 \leqslant g(\mathbf{Z}, Y):=$ $|Y-\eta(\mathbf{Z})| \leqslant|Y|+\eta(\mathbf{Z}) \mid \leqslant 1+1=2$. Therefore,

$$
\begin{aligned}
& \left|\sum_{j=1, \neq i}^{n} \delta_{j} W_{j}\left(\mathbf{X}_{i}, Y_{i}\right) g\left(\mathbf{Z}_{j}, Y_{j}\right)-E\left[g\left(\mathbf{Z}_{i}, Y_{i}\right) \mid \mathbf{X}_{i}, Y_{i}\right]\right| \\
& \leqslant\left|\sum_{j=1, \neq i}^{n} \delta_{j} W_{j}\left(\mathbf{X}_{i}, Y_{i}\right) g\left(\mathbf{Z}_{j}, Y_{j}\right)\right|+\left|E\left[g\left(\mathbf{Z}_{i}, Y_{i}\right) \mid \mathbf{X}_{i}, Y_{i}\right]\right| \leqslant 2+2=4,
\end{aligned}
$$

where

$$
W_{j}\left(\mathbf{X}_{i}, Y_{i}\right)=\frac{I\left\{Y_{j}=Y_{i}\right\} \mathcal{H}\left(\frac{\mathbf{x}_{j}-\mathbf{x}_{i}}{h_{n, \mathbf{x}}}\right)}{\sum_{j=1, \neq i}^{n} \delta_{j} I\left\{Y_{j}=Y_{i}\right\} \mathcal{H}\left(\frac{\mathbf{x}_{j}-\mathbf{X}_{i}}{h_{n, \mathbf{x}}}\right)}
$$

Since by our results in (5.17)

$$
p_{n i}=P\left\{\left|\sum_{j=1, \neq i}^{n} \delta_{j} W_{j}\left(\mathbf{X}_{i}, Y_{i}\right) g\left(\mathbf{Z}_{j}, Y_{j}\right)-E\left[g\left(\mathbf{Z}_{i}, Y_{i}\right) \mid \mathbf{X}_{i}, Y_{i}\right]\right|>\frac{t}{3}\right\},
$$

one only needs to focus on $0<t<12$ (when $t \geqslant 12$ then $p_{n i}=0$ ). The bound, in (5.23) in conjunction with (5.17), (5.15), and (5.16) implies that for every $\epsilon>0$, and $n$ large enough,

$$
P\left\{L_{n}\left(\widehat{\psi}_{n}\right)-L\left(\psi_{B}\right)>\epsilon\right\} \leqslant \mathcal{N}_{\infty}\left(\frac{\epsilon^{2}}{2048}, \mathcal{M}\right)\left[4 e^{-C_{7} n \epsilon^{4}}+8 n e^{-C_{8}(n-1) h_{n, \boldsymbol{x}}^{d} \epsilon^{4}}\right]+\delta_{n}\left(\frac{\epsilon}{8}\right) .
$$

Now, the theorem follows from the fact that $h_{n, \mathbf{x}} \rightarrow 0$, as $n \rightarrow \infty$ (and $\left.n h_{n, \mathbf{x}}^{d} \rightarrow \infty\right)$. 
Thus, if $\sum_{n=1}^{\infty} \delta_{n}\left(\frac{\epsilon}{8}\right)<\infty$, then $L_{n}\left(\widehat{\psi}_{n}\right) \stackrel{\text { a.s. }}{\rightarrow} L\left(\psi_{B}\right)$ from an application of the BorelCantelli lemma.

Proof of Lemma 5.2.8.

To prove this lemma, start by writing

$$
\begin{aligned}
E & {\left[\widehat{S}\left(g \mid \mathbf{X}_{i}, Y_{i}\right) \mid \mathbf{X}_{i}, Y_{i}\right]-S\left(g \mid \mathbf{X}_{i}, Y_{i}\right) } \\
= & \frac{1}{n-1} \sum_{j=1, \neq i}^{n}\left\{h_{n, \mathbf{x}}^{-d} E\left[\delta_{j} I\left\{Y_{j}=Y_{i}\right\} \mathcal{H}\left(\frac{\mathbf{X}_{j}-\mathbf{X}_{i}}{h_{n, \mathbf{x}}}\right) g\left(\mathbf{Z}_{j}, Y_{j}\right) \mid \mathbf{X}_{i}, Y_{i}\right]\right. \\
& \left.-S\left(g \mid \mathbf{X}_{i}, Y_{i}\right)\right\} \\
:= & \frac{1}{n-1} \sum_{j=1, \neq i}^{n} I_{n, i, j} .
\end{aligned}
$$

But $E\left[\delta_{j} I\left\{Y_{j}=Y_{i}\right\} \mathcal{H}\left(\frac{\mathbf{X}_{j}-\mathbf{X}_{i}}{h_{n, \mathbf{x}}}\right) g\left(\mathbf{Z}_{j}, Y_{j}\right) \mid \mathbf{X}_{i}, Y_{i}\right]$

$$
\begin{aligned}
=E\left\{I\left\{Y_{j}=Y_{i}\right\} \mathcal{H}\left(\frac{\mathbf{X}_{j}-\mathbf{X}_{i}}{h_{n, \mathbf{x}}}\right)\right. & \underbrace{E\left[\delta_{j} g\left(\mathbf{Z}_{j}, Y_{j}\right) \mid \mathbf{X}_{i}, Y_{i}, \mathbf{X}_{j}, Y_{j}\right]}_{=E\left[\delta_{j} g\left(\mathbf{Z}_{j}, Y_{j}\right) \mid \mathbf{X}_{j}, Y_{j}\right], \quad \text { (by independence) }} \mid \mathbf{X}_{i}, Y_{i}\} \\
& =\mu\left(g \mid \mathbf{X}_{j}, Y_{j}\right) .
\end{aligned}
$$

Therefore,

$$
\begin{aligned}
I_{n, i, j}= & h_{n, \mathbf{x}}^{-d} E\left[I\left\{Y_{j}=Y_{i}\right\} \mathcal{H}\left(\frac{\mathbf{X}_{j}-\mathbf{X}_{i}}{h_{n, \mathbf{x}}}\right) \mu\left(g \mid \mathbf{X}_{j}, Y_{j}\right) \mid \mathbf{X}_{i}, Y_{i}\right] \\
& \quad-f\left(\mathbf{X}_{i}\right) P\left\{Y=Y_{i} \mid Y_{i}\right\} \mu\left(g \mid \mathbf{X}_{i}, Y_{i}\right) \\
= & h_{n, \mathbf{x}}^{-d} E\left[\left(\mu\left(g \mid \mathbf{X}_{j}, Y_{j}\right)-\mu\left(g \mid \mathbf{X}_{i}, Y_{i}\right)\right) I\left\{Y_{j}=Y_{i}\right\} \mathcal{H}\left(\frac{\mathbf{X}_{j}-\mathbf{X}_{i}}{h_{n, \mathbf{x}}}\right) \mid \mathbf{X}_{i}, Y_{i}\right] \\
& \quad+E\left[\mu ( g | \mathbf { X } _ { i } , Y _ { i } ) \left\{h_{n, \mathbf{x}}^{-d} I\left\{Y_{j}=Y_{i}\right\} \mathcal{H}\left(\frac{\mathbf{X}_{j}-\mathbf{X}_{i}}{h_{n, \mathbf{x}}}\right)\right.\right. \\
& \left.\left.\quad-f\left(\mathbf{X}_{i}\right) P\left\{Y=Y_{i} \mid Y_{i}\right\}\right\} \mid \mathbf{X}_{i}, Y_{i}\right] \\
:= & I_{n, i, j}^{(1)}+I_{n, i, j}^{(2) .}
\end{aligned}
$$


Taylor expansion gives

$$
\left|I_{n, i, j}^{(1)}\right| \leqslant h_{n, \mathbf{x}}^{-d} E\left[\sum_{k=1}^{d}\left|\frac{\partial \mu\left(g \mid \mathbf{X}^{*}, Y_{i}\right)}{\partial x_{k}}\right| \cdot\left|X_{j k}-X_{i k}\right| \mathcal{H}\left(\frac{\mathbf{X}_{j}-\mathbf{X}_{i}}{h_{n, \mathbf{x}}}\right) \mid \mathbf{X}_{i}, Y_{i}\right]
$$

where $X_{j k}$ and $X_{i k}$ are the $k^{t h}$ components of the vectors $\mathbf{X}_{j}$ and $\mathbf{X}_{i}$ respectively, and $\mathbf{X}^{*}$ is on the interior of the line segment joining $\mathbf{X}_{i}$ and $\mathbf{X}_{j}$. Let $C_{\max }=\max _{i=0,1} \vee_{k=1}^{d}\left\|\left.\frac{\partial \mu(g \mid \mathbf{x}, i)}{\partial x_{k}}\right|_{\mathbf{x}=.}\right\|_{\infty}$, where the notation $\left\|\left.\frac{\partial \mu(g \mid \mathbf{x}, i)}{\partial x_{k}}\right|_{\mathbf{x}=.}\right\|_{\infty}$ stands for $\sup _{\mathbf{y} \in \mathcal{R}^{d}}\left|\frac{\partial \mu(g \mid \mathbf{x}, i)}{\partial x_{k}}\right|_{\mathbf{x}=\mathbf{y}} \mid$. Then by condition $\mathbf{C 4}, C_{\max }<\infty$, and consequently,

$$
\begin{aligned}
\left|I_{n, i, j}^{(1)}\right| & \leqslant C_{\max } \sum_{k=1}^{d} \int_{\mathcal{R}^{d}}\left|x_{k}-X_{i k}\right| h_{n, \mathbf{x}}^{-d} \mathcal{H}\left(\frac{\mathbf{x}-\mathbf{X}_{i}}{h_{n, \mathbf{x}}}\right) f(\mathbf{x}) d \mathbf{x} \\
& \leqslant C_{\max }\|f\|_{\infty} \sum_{k=1}^{d} \int_{\mathcal{R}^{d}} h_{n, \mathbf{x}}\left|u_{i}\right| \mathcal{H}(\mathbf{u}) d \mathbf{u} \\
& \leqslant \mid \text { const } \mid h_{n, \mathbf{x}} \quad \text { (by condition } \mathbf{C 3} \text { ) }
\end{aligned}
$$

As for the term $I_{n, i, j}^{(2)}$, first note that

$$
I_{n, i, j}^{(2)}=\mu\left(g \mid \mathbf{X}_{i}, Y_{i}\right) E\left[h_{n, \mathbf{x}}^{-d} I\left\{Y_{j}=Y_{i}\right\} \mathcal{H}\left(\frac{\mathbf{X}_{j}-\mathbf{X}_{i}}{h_{n, \mathbf{x}}}\right)-f\left(\mathbf{X}_{i}\right) P\left\{Y=Y_{i} \mid Y_{i}\right\} \mid \mathbf{X}_{i}, Y_{i}\right]
$$

Let $f_{y}(\mathbf{x})$ be the conditional density of $\mathbf{X}$, given class $y(y=0,1)$. Then

$$
\begin{aligned}
E[ & \left.h_{n, \mathbf{x}}^{-d} I\left\{Y_{j}=Y_{i}\right\} \mathcal{H}\left(\frac{\mathbf{X}_{j}-\mathbf{X}_{i}}{h_{n, \mathbf{x}}}\right) \mid \mathbf{X}_{i}, Y_{i}\right] \\
= & \int_{\mathcal{R}^{d} \times\{0,1\}} h_{n, \mathbf{x}}^{-d} I\left\{y=Y_{i}\right\} \mathcal{H}\left(\frac{\mathbf{u}-\mathbf{X}_{i}}{h_{n, \mathbf{x}}}\right) f(\mathbf{u}, y) d y d \mathbf{u} \\
& \quad\left(\text { where } f(\mathbf{u}, y)=f_{y}(\mathbf{u}) P(Y=y), \text { and } y=0,1\right) \\
= & \sum_{k=0,1} P\{Y=k\} I\left\{Y_{i}=k\right\} \int_{\mathcal{R}^{d}} h_{n, \mathbf{x}}^{-d} \mathcal{K}\left(\frac{\mathbf{u}-\mathbf{X}_{i}}{h_{n, \mathbf{x}}}\right) f_{k}(\mathbf{u}) d \mathbf{u} .
\end{aligned}
$$

Furthermore, noticing that $P\left\{Y=Y_{i} \mid Y_{i}\right\}=\sum_{k=0,1} P\{Y=k\} I\left\{Y_{i}=k\right\}$, and taking 
into account the fact that $\mathcal{H}(\cdot)$ integrates to 1 over $\mathcal{R}^{d}$ (by condition C3) one finds

$$
\begin{aligned}
I_{n, i, j}^{(2)}= & \mu\left(g \mid \mathbf{X}_{i}, Y_{i}\right) \sum_{k=0,1} P\{Y=k\} I\left\{Y_{i}=k\right\} \\
& \times\left[\int_{\mathcal{R}^{d}} h_{n, \mathbf{x}}^{-d} \mathcal{H}\left(\frac{\mathbf{u}-\mathbf{X}_{i}}{h_{n, \mathbf{x}}}\right)\left[f_{k}(\mathbf{u})-f_{k}\left(\mathbf{X}_{i}\right)\right] d \mathbf{u}\right] .
\end{aligned}
$$

Since $\left|\mu\left(g \mid \mathbf{X}_{i}, Y_{i}\right)\right| \leqslant 1$ and $\left|P(Y=k) I\left\{Y_{i}=k\right\}\right| \leqslant 1$, one obtains

$$
\begin{aligned}
\left|I_{n, i, j}^{(2)}\right| & \leqslant \sum_{k=0,1}\left|\int_{\mathcal{R}^{d}}\left[f_{k}\left(\mathbf{X}_{i}+h_{n, \mathbf{x}} \mathbf{v}\right)-f_{k}\left(\mathbf{X}_{i}\right)\right] \mathcal{H}(\mathbf{v}) d \mathbf{v}\right| \\
& \leqslant h_{n, \mathbf{x}} \sum_{k=0,1} \sum_{m=1}^{d} \| \partial f(\mathbf{x}) /\left.\partial x_{m}\right|_{\mathbf{x}=\cdot \|_{\infty}} \int_{\mathcal{R}^{d}}\left|v_{m}\right| \mathcal{H}(\mathbf{v}) d \mathbf{v} \quad \text { (via Taylor expansion) } \\
& =\mid \text { const } \mid h_{n, \mathbf{x}}, \quad \text { (by condition } \mathbf{C 2} \text { ) }
\end{aligned}
$$

This completes the proof of Lemma 5.2.8.

\subsection{Horvitz-Thompson-Type Estimators}

The assumption of total boundedness, imposed on the class $\mathcal{M}$ in the previous section is rather restrictive and undesirable. In practice supnorm covers or equivalently $\mathcal{N}_{\infty}(\cdot, \mathcal{M})$, can be very large and one is typically interested in carrying out the analysis based on weaker condition like $L_{p}$-norms, $p<\infty$ (see Definitions 1.4 .3 and 1.4.4). However, in practice the distribution of $\mathbf{Z}$ is unknown and one would like to use the empirical $L_{p}$-norm instead. (see Definitions 1.4.5 and 1.4.6.) That is, one is interested in finding bounds such as the one in Theorem 5.2 .7 , with $\mathcal{N}_{\infty}(\cdot, \mathcal{M})$ replaced by the expected value of the empirical covering number of the class $\mathcal{M}$ with respect to the empirical $L_{p}$-norm (in our study $p=1$ ). Unfortunately, in this case the dependence structure among the terms (which is caused by the kernel-based imputation approach of the previous section) prevents one from applying standard 
Dudley-Pollard, (Dudley (1978) and Pollard (1984)), symmetrization arguments to deal with the resulting empirical processes. To achieve our goal, we propose a Horvitz-Thompson-type estimation approach, which works by weighting the complete cases by the inverse of the missing data probabilities. There are some results related to the estimation and inference for the mean of a distribution (where the estimation is done via weighting of the complete cases by the inverse of missing data probabilities). Hirano et al. (2003) defined $\widehat{E Y}=n^{-1} \sum_{i=1}^{n} \frac{Y_{i} \delta_{i}}{p_{n}\left(\mathbf{X}_{i}\right)}$ which is essentially a corrected version of the naïve estimator based on the complete cases. These authors showed that weighting by the inverse of a nonparametric estimate of $p(\mathbf{X})$, rather than the true values, leads to an efficient estimate. The case where the missing data probabilities are known or can be parametrically modeled has been studied by Robins et al. (1994). In a more recent article, Wang et al. (2004) studied the situation where $Y$ follows the semiparametric model, $Y_{i}=\mathbf{X}_{i} \beta+g\left(\mathbf{T}_{i}\right)+\varepsilon_{i}$; here the covariates $\left(\mathbf{X}_{i}, \mathbf{T}_{i}\right) \in \mathcal{R}^{d} \times \mathcal{R}^{d^{*}}$ are observable and $Y_{i}$ could be missing at random, $\left(\varepsilon_{i}\right.$ are independent with $E\left(\varepsilon_{i} \mid \mathbf{X}_{i}, \mathbf{T}_{i}\right)=0$ ). In the present case of classification with missing covariates, start by defining

$$
\widehat{L}_{n, p}(\eta)=\frac{1}{n} \sum_{i=1}^{n} \frac{\delta_{i}}{p\left(\mathbf{X}_{i}, Y_{i}\right)}\left|Y_{i}-\eta\left(\mathbf{Z}_{i}\right)\right|^{2}
$$

Clearly, the above estimator is unbiased under the MAR assumption:

$$
\begin{aligned}
E\left[\widehat{L}_{n, p}(\eta)\right] & =E\left\{E\left[\frac{\delta}{p(\mathbf{X}, Y)}|Y-\eta(\mathbf{Z})|^{2} \mid \mathbf{Z}, Y\right]\right\} \\
& =E\left\{\frac{1}{p(\mathbf{X}, Y)}|Y-\eta(\mathbf{Z})|^{2} E(\delta \mid \mathbf{Z}, Y)\right\} \stackrel{\text { MAR }}{=} E|Y-\eta(\mathbf{Z})|^{2}
\end{aligned}
$$

Of course the estimator in (5.25) is useful when the function $p(\mathbf{x}, y)$ is known. If this is not the case, one may use a data-based estimator $\widehat{p}(\mathbf{x}, y)$ in which case $(5.25)$ 
becomes

$$
\widehat{L}_{n, \hat{p}}(\eta)=\frac{1}{n} \sum_{i=1}^{n} \frac{\delta_{i}}{\widehat{p}\left(\mathbf{X}_{i}, Y_{i}\right)}\left|Y_{i}-\eta\left(\mathbf{Z}_{i}\right)\right|^{2}
$$

Now let

$$
\eta_{n}= \begin{cases}\underset{\eta \in \mathcal{M}}{\operatorname{argmin}} \widehat{L}_{n, p}(\eta), & \text { if } p(\cdot, \cdot) \text { is known, } \\ \underset{\eta \in \mathcal{M}}{\operatorname{argmin}} \widehat{L}_{n, \hat{p}}(\eta), & \text { otherwise, }\end{cases}
$$

and define the corresponding classifier by

$$
\psi_{n}^{(H T)}(\mathbf{Z}, \delta)=\delta \psi_{n, 1}^{(H T)}(\mathbf{Z})+(1-\delta) \psi_{n, 0}(\mathbf{X}),
$$

where $\psi_{n, 1}^{(H T)}(\mathbf{Z})=I\left\{p_{1}(\mathbf{X}) \eta_{n}(\mathbf{Z})-p_{0}(\mathbf{X})\left(1-\eta_{n}(\mathbf{Z})\right)>0\right\}$ and $\psi_{n, 0}(\mathbf{X})=I\{(1-$ $\left.\left.p_{1}(\mathbf{X})\right) \lambda_{n}(\mathbf{X})-\left(1-p_{0}(\mathbf{X})\right)\left(1-\lambda_{n}(\mathbf{X})\right)>0\right\}$ for any estimator $\lambda_{n}$ of $\lambda$. How good is $\psi_{n}^{(H T)}$ ? The answer is partially buried in the following result. Fix the data $\mathcal{D}_{n}$ and for any $\epsilon>0$, let $\mathcal{N}_{1}\left(\epsilon, \mathcal{M},\left(\mathbf{Z}_{i}, \delta_{i}\right)_{i=1}^{n}\right)$ be the $\epsilon$-covering number of $\mathcal{M}$ with respect to the empirical $L_{1}$-norm, based on the complete cases. That is, $\mathcal{N}_{1}\left(\epsilon, \mathcal{M},\left(\mathbf{Z}_{i}, \delta_{i}\right)_{i=1}^{n}\right)$ is the cardinality of the smallest subclass of functions $\mathcal{M}_{\epsilon}=\left\{\eta_{1}, \cdots, \eta_{N(\epsilon)}: \mathcal{R}^{p} \rightarrow\right.$ $[0,1]\}$, with the property that for every $\eta \in \mathcal{M}$ there is a $\eta^{*} \in \mathcal{M}_{\epsilon}$ such that $\left(\sum_{j=1}^{n} \delta_{j}\right)^{-1} \sum_{i=1}^{n} \delta_{i}\left|\eta\left(\mathbf{Z}_{i}\right)-\eta^{*}\left(\mathbf{Z}_{i}\right)\right|<\epsilon$, provided that $\sum_{j=1}^{n} \delta_{j}>0$ (see Definition 1.4.6).

Theorem 5.3.1 Let $\psi_{n}^{(H T)}$ be as defined above. If the function $p(\mathbf{x}, y)=P\{\delta=$ $1 \mid \mathbf{X}=\mathbf{x}, Y=y\}$ is known then, under conditions $\mathbf{C 1}$ and $\mathbf{C 2}$, there is a $n_{0}$ such that for all $n>n_{0}$,

$$
P\left\{L_{n}\left(\psi_{n}^{(H T)}\right)-L\left(\psi_{B}\right)>\epsilon\right\} \leqslant 4 E\left[\mathcal{N}_{1}\left(\frac{p_{\min } \epsilon^{2}}{1024}, \mathcal{M},\left(\mathbf{Z}_{i}, \delta_{i}\right)_{i=1}^{n}\right)\right] e^{-C_{10} n \epsilon^{4}}+\delta_{n, 1}\left(\frac{\epsilon}{4}\right),
$$

where $C_{10}$ is a positive constant not depending on $n$, and

$$
\delta_{n, 1}(\cdot)=P\left\{E\left[\left|\lambda_{n}(\mathbf{X})-\lambda_{B}(\mathbf{X})\right| \mid \mathcal{D}_{n}\right]>\cdot\right\}
$$


The following proof is based on symmetrization arguments that go back to Dudley (1978) and Pollard (1984); such methods also appear in empirical process techniques, under "Rademacher Complexity", in machine learning literature; see, for example, Bartlett et al. (2005) and Bartlett et al. (2002).

\section{Proof of Theorem 5.3.1.}

Let $\mathcal{G} \equiv \mathcal{G}_{\mathcal{M}}$ be as in (5.14) and $\mu(g)=E g(\mathbf{Z}, Y):=E|Y-\eta(\mathbf{Z})|^{2}$. Also put $\mu_{n, p}(g)=\frac{1}{n} \sum_{i=1}^{n} \frac{\delta_{i}}{p\left(\mathbf{X}_{i}, Y_{i}\right)} g\left(\mathbf{Z}_{i}, Y_{i}\right)$. Then, as in the proof of Theorem 5.2.7, one has

$$
L_{n}\left(\psi_{n}^{(H T)}\right)-L\left(\psi_{B}\right) \leqslant 2 \sqrt{2 \sup _{g \in \mathcal{G}}\left|\mu_{n, p}(g)-\mu(g)\right|}+2 E\left[\left|\lambda_{n}(\mathbf{X})-\lambda(\mathbf{X})\right| \mid \mathcal{D}_{n}\right]
$$

In other words, $\forall \epsilon>0$,

$$
\begin{aligned}
P\left\{L_{n}\left(\psi_{n}^{(H T)}\right)-L\left(\psi_{B}\right)>\epsilon\right\} & \leqslant P\left\{\sup _{g \in \mathcal{G}}\left|\mu_{n, p}(g)-\mu(g)\right|>\frac{\epsilon^{2}}{32}\right\} \\
& +P\left\{E\left[\lambda_{n}(\mathbf{X})-\lambda_{B}(\mathbf{X})|| \mathcal{D}_{n}\right]>\frac{\epsilon}{4}\right\}
\end{aligned}
$$

We shall use symmetrization arguments to finish the proof. Fix the data $\mathcal{D}_{n}$. If $\sup _{g \in \mathcal{G}}\left|\mu_{n, p}(g)-\mu(g)\right|>\frac{\epsilon^{2}}{32}$ then there is a $g_{\epsilon} \in \mathcal{G}$ (which will depend on the data $\left.\mathcal{D}_{n}\right)$ such that $\left|\mu_{n, p}\left(g_{\epsilon}\right)-\mu\left(g_{\epsilon} \mid \mathcal{D}_{n}\right)\right|>\frac{\epsilon^{2}}{32}$, where $\mu\left(g_{\epsilon} \mid \mathcal{D}_{n}\right)=E\left[g_{\epsilon}(\mathbf{Z}, Y) \mid \mathcal{D}_{n}\right]$. Let $\mathcal{D}_{n}^{\prime}=\left\{\left(\mathbf{Z}_{1}^{\prime}, Y_{1}^{\prime}, \delta_{1}^{\prime}\right), \cdots,\left(\mathbf{Z}_{n}^{\prime}, Y_{n}^{\prime}, \delta_{n}^{\prime}\right)\right\}$ be an i.i.d. ghost sample, where $\left(\mathbf{Z}_{i}^{\prime}, Y_{i}^{\prime}, \delta_{i}^{\prime}\right) \stackrel{\text { i.i.d. }}{=}$ $\left(\mathbf{Z}_{1}, Y_{1}, \delta_{1}\right)$. Put

$$
\mu_{n, p}^{\prime}(g)=\frac{1}{n} \sum_{i=1}^{n} \frac{\delta_{i}^{\prime}}{p\left(\mathbf{X}_{i}^{\prime}, Y_{i}^{\prime}\right)} g\left(\mathbf{Z}_{i}^{\prime}, Y_{i}^{\prime}\right)
$$


and observe that

$$
\begin{aligned}
& P\left\{\left|\mu_{n, p}^{\prime}\left(g_{\epsilon}\right)-\mu\left(g_{\epsilon} \mid \mathcal{D}_{n}\right)\right|<\frac{\epsilon^{2}}{64} \mid \mathcal{D}_{n}\right\} \\
& =1-P\left\{\left|\mu_{n, p}^{\prime}\left(g_{\epsilon}\right)-\mu\left(g_{\epsilon} \mid \mathcal{D}_{n}\right)\right| \geqslant \frac{\epsilon^{2}}{64} \mid \mathcal{D}_{n}\right\} \\
& \geqslant 1-\sup _{g \in \mathcal{G}} P\left\{\left|\frac{1}{n} \sum_{i=1}^{n} \frac{\delta^{\prime}}{p\left(\mathbf{X}_{i}^{\prime}, Y_{i}^{\prime}\right)} g\left(\mathbf{Z}_{i}^{\prime}, Y_{i}^{\prime}\right)-\mu(g)\right| \geqslant \frac{\epsilon^{2}}{64}\right\} \\
& \geqslant 1-\frac{64^{2}}{n \epsilon^{4}} \sup _{g \in \mathcal{G}} \operatorname{Var}\left(\frac{\delta_{1}}{p\left(\mathbf{X}_{1}, Y_{1}\right)} g\left(\mathbf{Z}_{1}, Y_{1}\right)\right) \\
& \geqslant 1-\frac{64^{2}}{n \epsilon^{4}} \cdot \frac{\|g\|_{\infty}^{2}}{p_{\text {min }}^{2}} \\
& >\frac{1}{2}, \text { for } n \text { large enough. }
\end{aligned}
$$

Therefore, for $n$ large,

$$
\begin{aligned}
\frac{1}{2} & <P\left\{\left|\mu_{n, p}^{\prime}\left(g_{\epsilon}\right)-\mu\left(g_{\epsilon} \mid \mathcal{D}_{n}\right)\right|<\frac{\epsilon^{2}}{64} \mid \mathcal{D}_{n}\right\} \\
& \leqslant P\left\{-\left|\mu_{n, p}^{\prime}\left(g_{\epsilon}\right)-\mu_{n, p}\left(g_{\epsilon}\right)\right|+\left|\mu_{n, p}\left(g_{\epsilon}\right)-\mu\left(g_{\epsilon} \mid \mathcal{D}_{n}\right)\right|<\frac{\epsilon^{2}}{64} \mid \mathcal{D}_{n}\right\} \\
& \leqslant P\left\{\left|\mu_{n, p}^{\prime}\left(g_{\epsilon}\right)-\mu_{n, p}\left(g_{\epsilon}\right)\right|>\frac{\epsilon^{2}}{64} \mid \mathcal{D}_{n}\right\} \\
& \leqslant P\left\{\sup _{g \in \mathcal{G}}\left|\mu_{n, p}^{\prime}(g)-\mu_{n, p}(g)\right|>\frac{\epsilon^{2}}{64} \mid \mathcal{D}_{n}\right\} .
\end{aligned}
$$

Now, the far left and far right sides of (5.28) do not depend on any specific $g_{\epsilon}$, and the chain of inequalities between them are valid on the set $\left\{\sup _{g \in \mathcal{G}}\left|\mu_{n, p}(g)-\mu(g)\right|>\frac{\epsilon^{2}}{32}\right\}$. Thus, multiplying the two far sides of $(5.28)$ by $I\left\{\sup _{g \in \mathcal{G}}\left|\mu_{n, p}(g)-\mu(g)\right|>\frac{\epsilon^{2}}{32}\right\}$ and taking expectation w.r.t. the distribution of $\mathcal{D}_{n}$, one finds 


$$
\begin{aligned}
& \frac{1}{2} P\left\{\sup _{g \in \mathcal{G}}\left|\mu_{n, p}(g)-\mu(g)\right|>\frac{\epsilon^{2}}{32}\right\} \\
& \leqslant P\left\{\sup _{g \in \mathcal{G}}\left|\mu_{n, p}^{\prime}(g)-\mu_{n, p}(g)\right|>\frac{\epsilon^{2}}{64}\right\} \\
& \quad=P\left\{\sup _{g \in \mathcal{G}} \frac{1}{n}\left|\sum_{i=1}^{n}\left[\frac{\delta_{i} g\left(\mathbf{Z}_{i}, Y_{i}\right)}{p\left(\mathbf{X}_{i}, Y_{i}\right)}-\frac{\delta_{i}^{\prime} g\left(\mathbf{Z}_{i}^{\prime}, Y_{i}^{\prime}\right)}{p\left(\mathbf{X}_{i}^{\prime}, Y_{i}^{\prime}\right)}\right]\right|>\frac{\epsilon^{2}}{64}\right\} .
\end{aligned}
$$

Let $R_{1}, \cdots, R_{n}$ be i.i.d. with $P\left(R_{i}=-1\right)=\frac{1}{2}=P\left(R_{i}=+1\right)$; here $R_{1}, \cdots, R_{n}$ are independent of both $\mathcal{D}_{n}$ and $\mathcal{D}_{n}^{\prime}$. Then

$$
\begin{aligned}
& P\left\{\sup _{g \in \mathcal{G}}\left|\mu_{n, p}(g)-\mu(g)\right|>\frac{\epsilon^{2}}{32}\right\} \\
& \leqslant 2 P\left\{\sup _{g \in \mathcal{G}} \frac{1}{n}\left|\sum_{i=1}^{n} R_{i}\left[\frac{\delta_{i} g\left(\mathbf{Z}_{i}, Y_{i}\right)}{p\left(\mathbf{X}_{i}, Y_{i}\right)}-\frac{\delta_{i}^{\prime} g\left(\mathbf{Z}_{i}^{\prime}, Y_{i}^{\prime}\right)}{p\left(\mathbf{X}_{i}^{\prime}, Y_{i}^{\prime}\right)}\right]\right|>\frac{\epsilon^{2}}{64}\right\} \\
& \leqslant 4 P\left\{\sup _{g \in \mathcal{G}} \frac{1}{n}\left|\sum_{i=1}^{n} R_{i} \delta_{i} g\left(\mathbf{Z}_{i}, Y_{i}\right) / p\left(\mathbf{X}_{i}, Y_{i}\right)\right|>\frac{\epsilon^{2}}{128}\right\} .
\end{aligned}
$$

Fix $\mathcal{D}_{n}$ and let $\mathcal{G}_{n, 256^{-1} \epsilon^{2} p_{\min }}$ be an empirical $L_{1} \frac{\epsilon^{2} p_{\min }}{256}$-cover of $\mathcal{G}$ based on the complete cases only. I.e., $\forall g \in \mathcal{G}$, there is a $g^{*} \in \mathcal{G}_{n, 256^{-1} \epsilon^{2} p_{\min }}$ such that $\frac{1}{n} \sum_{i=1}^{n} \delta_{i}\left|g\left(\mathbf{Z}_{i}, Y_{i}\right)-g^{*}\left(\mathbf{Z}_{i}, Y_{i}\right)\right|<\frac{\epsilon^{2} p_{\min }}{256}$. Let $\mathcal{N}_{1}\left(\frac{\epsilon^{2} p_{\min }}{256}, \mathcal{G},\left(\mathbf{Z}_{i}, Y_{i}, \delta_{i}\right)_{i=1}^{n}\right)$ be the $\frac{\epsilon^{2} p_{\min }}{256}$-covering number of $\mathcal{G}$. Then for some $g^{*} \in \mathcal{G}_{n, 256^{-1} \epsilon^{2} p_{\min }}$,

$$
\begin{aligned}
\frac{1}{n}\left|\sum_{i=1}^{n} R_{i} \delta_{i} g\left(\mathbf{Z}_{i}, Y_{i}\right) / p\left(\mathbf{X}_{i}, Y_{i}\right)\right| & \leqslant \frac{1}{n}\left|\sum_{i=1}^{n} \frac{R_{i} \delta_{i}}{p\left(\mathbf{X}_{i}, Y_{i}\right)}\left[g\left(\mathbf{Z}_{i}, Y_{i}\right)-g^{*}\left(\mathbf{Z}_{i}, Y_{i}\right)\right]\right| \\
& +\frac{1}{n}\left|\sum_{i=1}^{n} R_{i} \delta_{i} g^{*}\left(\mathbf{Z}_{i}, Y_{i}\right) / p\left(\mathbf{X}_{i}, Y_{i}\right)\right| \\
\leqslant & \frac{1}{p_{\min }} \cdot \frac{\epsilon^{2} p_{\min }}{256}+\frac{1}{n}\left|\sum_{i=1}^{n} R_{i} \delta_{i} g^{*}\left(\mathbf{Z}_{i}, Y_{i}\right) / p\left(\mathbf{X}_{i}, Y_{i}\right)\right| .
\end{aligned}
$$


Therefore,

$$
\begin{aligned}
\text { R.H.S. of }(5.29) & \leqslant 4 E P\left\{\sup _{g \in \mathcal{G}_{n, 256^{-1} \epsilon^{2} p_{\min }}}\left|\frac{1}{n} \sum_{i=1}^{n} R_{i} \delta_{i} g\left(\mathbf{Z}_{i}, Y_{i}\right) / p\left(\mathbf{X}_{i}, Y_{i}\right)\right|>\frac{\epsilon^{2}}{256} \mid \mathcal{D}_{n}\right\} \\
& \leqslant 4 E\left[\mathcal{N}_{1}\left(\frac{\epsilon^{2} p_{\min }}{256}, \mathcal{G},\left(\mathbf{Z}_{i}, Y_{i}, \delta_{i}\right)_{i=1}^{n}\right)\right] e^{-\frac{2 n\left(\epsilon^{2} / 256\right)^{2}}{4\left(\|g\| \infty / p_{\min }\right)^{2}}} .
\end{aligned}
$$

But $\mathcal{N}_{1}\left(\epsilon^{2} p_{\min } / 256, \mathcal{G},\left(\mathbf{Z}_{i}, Y_{i}, \delta_{i}\right)_{i=1}^{n}\right) \leqslant \mathcal{N}_{1}\left(\epsilon^{2} p_{\min } / 1024, \mathcal{M},\left(\mathbf{Z}_{i}, \delta_{i}\right)_{i=1}^{n}\right)$, which completes the proof of Theorem 5.3.1.

Next, in order to study the case where the function $p(\mathbf{x}, y)$ is unknown, we consider two possible estimators to be used in $\widehat{L}_{n, \hat{p}}(\eta)$, (see expression (5.26)). Our first estimator is the kernel-type regression estimator.

$$
\widehat{p}\left(\mathbf{X}_{i}, Y_{i}\right)=\frac{\sum_{j=1, \neq i}^{n} \delta_{j} \mathcal{J}\left(\frac{\mathbf{x}_{j}-\mathbf{x}_{i}}{h_{n}}\right) I\left\{Y_{i}=Y_{j}\right\}}{\sum_{j=1, \neq i}^{n} \mathcal{J}\left(\frac{\mathbf{x}_{j}-\mathbf{X}_{i}}{h_{n}}\right) I\left\{Y_{i}=Y_{j}\right\}}
$$

The resulting classifier is

$$
\psi_{n_{\hat{p}}}^{(H T)}(\mathbf{Z}, \delta)=\delta \psi_{n_{\hat{p}}, \mathbf{1}}^{(H T)}(\mathbf{Z})+(1-\delta) \psi_{n_{\hat{p}}, 0}(\mathbf{X})
$$

where $\psi_{n_{\hat{p}}, 1}(\mathbf{Z})=I\left\{\widehat{p}_{1}(\mathbf{X}) \eta_{n}(\mathbf{Z})-\widehat{p}_{0}(\mathbf{X})\left(1-\eta_{n}(\mathbf{Z})\right)>0\right\}$ with $\eta_{n}=\underset{\eta \in \mathcal{M}}{\operatorname{argmin}} \widehat{L}_{n, \hat{p}}(\eta)$, and $\psi_{n_{\hat{p}}, 0}(\mathbf{X})=I\left\{\left(1-\widehat{p}_{1}(\mathbf{X})\right) \lambda_{n}(\mathbf{X})-\left(1-\widehat{p}_{0}(\mathbf{X})\right)\left(1-\lambda_{n}(\mathbf{X})>0\right\}\right.$ as before. To study the performance of $\psi_{n_{\hat{p}}}^{(H T)}$, we first state the following condition.

$\mathbf{C}^{\prime} 4$. The partial derivatives $\frac{\partial}{\partial x_{i}} p(\mathbf{x}, y)$, where $i=1, \cdots, d$, and $y=0$ or 1 exist and are bounded on the compact support of $f$, uniformly in $\mathbf{x}$.

Theorem 5.3.2 Suppose that conditions $\mathbf{C 1}, \mathbf{C 2}, \mathbf{C 3}$, and $\mathbf{C}^{\prime} \mathbf{4}$ hold. Then $\forall \epsilon>0$, 
there is an $n_{0}$ such that for $n>n_{0}$

$$
\begin{aligned}
& P\left\{L_{n}\left(\psi_{n_{\hat{p}}}^{(H T)}\right)-L\left(\psi_{B}\right)>\epsilon\right\} \\
\leqslant & 4 E\left[\mathcal{N}_{1}\left(\frac{\epsilon^{2} p_{\min }}{2048}, \mathcal{G},\left(\mathbf{Z}_{i}, Y_{i}, \delta_{i}\right)_{i=1}^{n}\right)\right] e^{-C_{11} n \epsilon^{4}}+4 n e^{-C_{12} n h_{n}^{d} \epsilon^{4}}+4 n e^{-C_{13} n h_{n}^{d}} \\
& +4 e^{-C_{14} n \epsilon^{2}}+\delta_{n, 1}\left(\frac{\epsilon}{8}\right)
\end{aligned}
$$

where the positive constants $C_{11}$ to $C_{14}$ do not depend on $n$, and $\delta_{n, 1}(\cdot)$ is as in (5.27).

\section{Proof.}

Let $\mathcal{G}$ be as in (5.14), and for every $g \in \mathcal{G}$ put $\mu_{n, \hat{p}}(g)=\frac{1}{n} \sum_{i=1}^{n} \frac{\delta_{i}}{\hat{p}\left(\mathbf{X}_{i}, Y_{i}\right)} g\left(\mathbf{Z}_{i}, Y_{i}\right)$, and $\mu(g)=E g(\mathbf{Z}, Y)$. Since

$$
\frac{\delta_{i}}{\widehat{p}\left(\mathbf{X}_{i}, Y_{i}\right)} g\left(\mathbf{Z}_{i}, Y_{i}\right)=-\frac{\delta_{i} g\left(\mathbf{Z}_{i}, Y_{i}\right)}{\widehat{p}\left(\mathbf{X}_{i}, Y_{i}\right)}\left[\frac{\widehat{p}\left(\mathbf{X}_{i}, Y_{i}\right)}{p\left(\mathbf{X}_{i}, Y_{i}\right)}-1\right]+\frac{\delta_{i}}{p\left(\mathbf{X}_{i}, Y_{i}\right)} g\left(\mathbf{Z}_{i}, Y_{i}\right),
$$

one finds (using Corollary 2.3.1),

$$
\begin{aligned}
P & \left\{L_{n}\left(\psi_{n_{\hat{p}}}^{(H T)}\right)-L\left(\psi_{B}\right)>\epsilon\right\} \\
\leqslant P & \left\{\sup _{g \in \mathcal{G}}\left|\mu_{n, \hat{p}}(g)-\mu(g)\right|>\frac{\epsilon^{2}}{128}\right\} \\
& +P\left\{E\left[\left|\widehat{p}_{1}(\mathbf{X})-p_{1}(\mathbf{X})\right| \mid \mathcal{D}_{n}\right]>\frac{\epsilon}{8}\right\}+P\left\{E\left[\left|\widehat{p}_{0}(\mathbf{X})-p_{0}(\mathbf{X})\right| \mid \mathcal{D}_{n}\right]>\frac{\epsilon}{8}\right\} \\
& +\delta_{n, 1}\left(\frac{\epsilon}{8}\right) \\
\leqslant \quad & \left\{\sup _{g \in \mathcal{G}}\left|\mu_{n, p}(g)-\mu(g)\right|>\frac{\epsilon^{2}}{256}\right\}+P\left\{\frac{1}{n} \sum_{i=1}^{n}\left|\frac{1}{\widehat{p}\left(\mathbf{X}_{i}, Y_{i}\right)}\right| \cdot\left|\frac{\widehat{p}\left(\mathbf{X}_{i}, Y_{i}\right)}{p\left(\mathbf{X}_{i}, Y_{i}\right)}-1\right|>\frac{\epsilon^{2}}{256}\right\} \\
& +\left[P\left\{E\left[\left|\widehat{p}_{1}(\mathbf{X})-p_{1}(\mathbf{X})\right| \mid \mathcal{D}_{n}\right]>\frac{\epsilon}{8}\right\}+P\left\{E\left[\left|\widehat{p}_{0}(\mathbf{X})-p_{0}(\mathbf{X})\right| \mid \mathcal{D}_{n}\right]>\frac{\epsilon}{8}\right\}\right] \\
& +\delta_{n, 1}\left(\frac{\epsilon}{8}\right) \\
:=\pi_{n, 1} & +\pi_{n, 2}(\widehat{p})+\pi_{n, 3}(\widehat{p})+\delta_{n, 1}\left(\frac{\epsilon}{8}\right) .
\end{aligned}
$$


But, by the arguments leading to (5.30) in the proof of Theorem 5.3.1, one finds

$$
\pi_{n, 1} \leqslant 4 E\left[\mathcal{N}_{1}\left(\frac{p_{\min } \epsilon^{2}}{2048}, \mathcal{G},\left(\mathbf{Z}_{i}, Y_{i}, \delta_{i}\right)_{i=1}^{n}\right)\right] e^{-C_{11} n \epsilon^{4}}
$$

for some positive constant $C_{11}$ that does not depend on $n$, As for the term $\pi_{n, 2}(\widehat{p})$, first observe that

$$
\begin{aligned}
\pi_{n, 2}(\widehat{p}) \leqslant P & \left\{\left[\frac{1}{n} \sum_{i=1}^{n}\left|\frac{1}{\widehat{p}\left(\mathbf{X}_{i}, Y_{i}\right)}\right| \cdot\left|\frac{\widehat{p}\left(\mathbf{X}_{i}, Y_{i}\right)}{p\left(\mathbf{X}_{i}, Y_{i}\right)}-1\right|>\frac{\epsilon^{2}}{64}\right] \bigcap \bigcap_{i=1}^{n}\left[\widehat{p}\left(\mathbf{X}_{i}, Y_{i}\right) \geqslant \frac{p_{\min }}{2}\right]\right\} \\
& +P\left\{\bigcup_{i=1}^{n}\left[\widehat{p}\left(\mathbf{X}_{i}, Y_{i}\right)<\frac{p_{\min }}{2}\right]\right\}, \\
\leqslant & \sum_{i=1}^{n} P\left\{\frac{2}{p_{\min }^{2}}\left|\widehat{p}\left(\mathbf{X}_{i}, Y_{i}\right)-p\left(\mathbf{X}_{i}, Y_{i}\right)\right|>\frac{\epsilon^{2}}{64}\right\}+\sum_{i=1}^{n} P\left\{\widehat{p}\left(\mathbf{X}_{i}, Y_{i}\right)<\frac{p_{\min }}{2}\right\} .
\end{aligned}
$$

However, arguments similar to those used in the derivation (5.22) can be used to show that for every $\gamma>0$ and $n$ large enough,

$$
P\left\{\left|\widehat{p}\left(\mathbf{X}_{i}, Y_{i}\right)-p\left(\mathbf{X}_{i}, Y_{i}\right)\right|>\gamma \mid \mathbf{X}_{i}, Y_{i}\right\} \leqslant 4 e^{-C_{16} n h_{n, x}^{d} \gamma^{2}}
$$

where $C_{15}>0$ does not depend on $n$. Since $P\left\{\widehat{p}\left(\mathbf{X}_{i}, Y_{i}\right)<p_{\min } / 2\right\} \leqslant P\left\{\mid \widehat{p}\left(\mathbf{X}_{i}, Y_{i}\right)-\right.$ $\left.p\left(\mathbf{X}_{i}, Y_{i}\right) \mid>p_{\min } / 2\right\}$, one finds

$$
\pi_{n, 2}(\widehat{p}) \leqslant 4 n e^{-C_{12} n h_{n}^{d} \epsilon^{4}}+4 n e^{-C_{13} n h_{n}^{d}}
$$

The theorem now follows upon noticing that $\pi_{n, 3}(\widehat{p})$ can be bounded ( using the same arguments that lead to (5.8)) by

$$
\pi_{n, 3}(\widehat{p})<4 e^{-C_{14} n \epsilon^{2}}
$$


Clearly the bound in Theorem 5.3.2 is not as tight as the one in Theorem 5.3.1 in the sense that the effective sample size appearing in the exponential function is $n h_{n}^{d}$ rather than $n$. This is the price to pay for replacing $p(\mathbf{x}, y)$ by its kernel estimator.

To motivate our second estimator, we shall assume that whether an observation is missing does not depend on the class $Y$. This is an observation is missing at random in the sense that

$$
P\{\delta=1 \mid \mathbf{X}, \mathbf{V}, Y\}=P\{\delta=1 \mid \mathbf{X}\}
$$

Now, suppose that one knows in advance that the unknown $p$ belongs to some known class $\mathcal{P}$ of functions $p: \mathcal{R}^{d} \rightarrow\left[p_{\min }, 1\right]$, where $p_{\min }=\inf _{\mathbf{x}} P\{\delta=1 \mid \mathbf{X}=\mathbf{x}\}$, is assumed to be known. Then one may consider the least-squares estimator

$$
\widetilde{p}=\inf _{p \in \mathcal{P}} \frac{1}{n} \sum_{i=1}^{n}\left|\delta_{i}-p\left(\mathbf{X}_{i}\right)\right|^{2},
$$

Also, let $\widetilde{\eta}_{n}=\underset{\eta \in \mathcal{M}}{\operatorname{argmin}} \widehat{L}_{n, \tilde{p}}(\eta)$, with $\widetilde{p}$ as above, and define the corresponding classifier

$$
\psi_{n_{\tilde{p}}}^{(H T)}(\mathbf{Z}, \delta)=\delta \psi_{n_{\tilde{p}}, \mathbf{1}}(\mathbf{Z})+(1-\delta) \psi_{n_{\tilde{p}}, 0}(\mathbf{X})
$$

where $\psi_{n_{\tilde{p}}, 1}(\mathbf{Z})=I\left\{\widetilde{p}_{1}(\mathbf{X}) \widetilde{\eta}_{n}(\mathbf{Z})-\widetilde{p}_{0}(\mathbf{X})\left(1-\widetilde{\eta}_{n}(\mathbf{Z})\right)>0\right\}$ and $\psi_{n_{\tilde{p}}, 0}(\mathbf{X})=I\{(1-$ $\left.\left.\widetilde{p}_{\mathbf{1}}(\mathbf{X})\right) \lambda_{n}(\mathbf{X})-\left(1-\widetilde{p}_{0}(\mathbf{X})\right)\left(1-\lambda_{n}(\mathbf{X})\right)>0\right\}$, for any estimator $\lambda_{n}$ of $\lambda$. The following theorem provides performance bound for $\psi_{n_{\tilde{p}}}^{(H T)}$.

Theorem 5.3.3 Let $\widetilde{p}$ be as above and suppose that conditions $\mathbf{C 1}$ and C2 hold. Then $\forall \epsilon>0$ there is an $n_{0}$ such that for all $n>n_{0}$,

$$
\begin{aligned}
P\left\{L_{n}\left(\psi_{n_{\tilde{p}}}^{(H T)}\right)-L\left(\psi_{B}\right)>\epsilon\right\} & \\
\leqslant 16 \Delta_{n}\left(\epsilon^{2}, \mathcal{P}, \mathcal{G}\right) e^{-C_{16} n \epsilon^{4}} & +8 E\left[\mathcal{N}_{1}\left(\frac{p_{\min }^{4}}{(512)^{2}(16)} \epsilon^{4}, \mathcal{P},\left(\mathbf{X}_{i}\right)_{i=1}^{n}\right)\right] e^{-C_{17} n \epsilon^{8}} \\
& +\delta_{n, 1}\left(\frac{\epsilon}{8}\right)
\end{aligned}
$$


where

$$
\begin{aligned}
\Delta_{n}\left(\epsilon^{2}, \mathcal{P}, \mathcal{G},\right)=E[ & \mathcal{N}_{1}\left(\frac{p_{\text {min }} \epsilon^{2}}{2048}, \mathcal{G},\left(\mathbf{Z}_{i}, Y_{i}, \delta_{i}\right)_{i=1}^{n}\right) \bigvee \mathcal{N}_{1}\left(\frac{p_{\min }^{2} \epsilon^{2}}{4096}, \mathcal{P},\left(\mathbf{X}_{i}\right)_{i=1}^{n}\right) \\
& \left.\bigvee \mathcal{N}_{1}\left(\frac{\epsilon^{2}}{(64)(16)}, \mathcal{P},\left(\mathbf{X}_{i}\right)_{i=1}^{n}\right)\right] .
\end{aligned}
$$

Here the constants $C_{16}$ and $C_{17}$ are positive and do not depend on $n$ and $\delta_{n, 1}(\cdot)$ is defined in (5.27).

\section{Proof.}

Replace $\widehat{p}$ with $\widetilde{p}$ in the proof of Theorem 5.3.2 and note that (see (5.31))

$$
P\left\{L_{n}\left(\psi_{n_{\tilde{p}}}^{(H T)}\right)-L\left(\psi_{B}\right)>\epsilon\right\} \leqslant \pi_{n, 1}+\pi_{n, 2}(\widetilde{p})+\pi_{n, 3}(\widetilde{p})+\delta_{n, 1}\left(\frac{\epsilon}{8}\right),
$$

where $\pi_{n, 1}$ is bounded according to $(5.32)$. As for the term $\pi_{n, 2}(\widetilde{p})$, observe that

$$
\begin{aligned}
\pi_{n, 2}(\widetilde{p}) \leqslant & P\left\{\frac{1}{n p_{\min }^{2}} \sum_{i=1}^{n}\left|\widetilde{p}\left(\mathbf{X}_{i}\right)-p\left(\mathbf{X}_{i}\right)\right|>\frac{\epsilon^{2}}{256}\right\} \\
\leqslant & P\left\{\sup _{p^{\prime} \in \mathcal{P}}\left|\frac{1}{n} \sum_{i=1}^{n}\right| p^{\prime}\left(\mathbf{X}_{i}\right)-p\left(\mathbf{X}_{i}\right)|-E| p^{\prime}(\mathbf{X})-p(\mathbf{X})||>\frac{p_{\min }^{2} \epsilon^{2}}{512}\right\} \\
& +P\left\{E\left[|\widetilde{p}(\mathbf{X})-p(\mathbf{X})| \mid \mathcal{D}_{n}\right]>\frac{p_{\min }^{2} \epsilon^{2}}{512}\right\} \\
:= & \pi_{n, 2}(i)+\pi_{n, 2}(i i) .
\end{aligned}
$$

The term $\pi_{n, 2}(i)$ can be bounded using standard results from the empirical processes theory (see, for example, Pollard (1984; page 26-27) or Devroye et al. (1996; Theorem 29.1)) according to

$$
\pi_{n, 2}(i) \leqslant 8 E\left[\mathcal{N}_{1}\left(\frac{p_{\min }^{2}}{4096} \epsilon^{2}, \mathcal{P},\left(\mathbf{X}_{i}\right)_{i=1}^{n}\right)\right] e^{-\frac{n p_{\min }^{4} \epsilon^{4}}{(512)^{2}(128)}}
$$

To deal with $\pi_{n, 2}(i i)$, let

$$
\widehat{L}(p)=\frac{1}{n} \sum_{i=1}^{n}\left|p\left(\mathbf{X}_{i}\right)-\delta_{i}\right|^{2}
$$


and note that

$$
\begin{aligned}
\pi_{n, 2}(i i) \leqslant P & \left\{E\left[|\widetilde{p}(\mathbf{X})-p(\mathbf{X})|^{2} \mid \mathcal{D}_{n}\right]>\frac{p_{\min }^{4} \epsilon^{4}}{512^{2}}\right\} \\
& \text { (Cauchy-Schwarz inequality) } \\
= & P\left\{E\left[|\widetilde{p}(\mathbf{X})-\delta|^{2} \mid \mathcal{D}_{n}\right]-E|p(\mathbf{X})-\delta|^{2}>\frac{p_{\min }^{4} \epsilon^{4}}{512^{2}}\right\} \\
(\text { since } p(\mathbf{X})=E(\delta \mid \mathbf{X})) & \\
\leqslant & P\left\{2 \sup _{p^{\prime} \in \mathcal{P}}\left|\widehat{L}\left(p^{\prime}\right)-E\right| p^{\prime}(\mathbf{X})-\left.\delta\right|^{2} \mid>\frac{p_{\min }^{4} \epsilon^{4}}{512^{2}}\right\},
\end{aligned}
$$

where we have used the fact that

$$
\begin{aligned}
& E\left[|\widetilde{p}(\mathbf{X})-\delta|^{2} \mid \mathcal{D}_{n}\right]-E|p(\mathbf{X})-\delta|^{2} \\
& =E\left[|\widetilde{p}(\mathbf{X})-\delta|^{2} \mid \mathcal{D}_{n}\right]-\inf _{p^{\prime} \in \mathcal{P}} E\left|p^{\prime}(\mathbf{X})-\delta\right|^{2} \\
& =\sup _{p^{\prime} \in \mathcal{P}}\left\{E\left[|\widetilde{p}(\mathbf{X})-\delta|^{2} \mid \mathcal{D}_{n}\right]-\widehat{L}(\widetilde{p})+\widehat{L}(\widetilde{p})-\widehat{L}\left(p^{\prime}\right)+\widehat{L}\left(p^{\prime}\right)-E\left|p^{\prime}(\mathbf{X})-\delta\right|^{2}\right\}
\end{aligned}
$$$$
\text { (because } \widehat{L}(\widetilde{p})-\widehat{L}\left(p^{\prime}\right) \leqslant 0 \text { by the definition of } \tilde{p} \text { ) }
$$$$
\leqslant 2 \sup _{p^{\prime} \in \mathcal{P}}\left|\widehat{L}\left(p^{\prime}\right)-E\right| p^{\prime}(\mathbf{X})-\left.\delta\right|^{2} \mid
$$

Hence

$$
\pi_{n, 2}(i i) \leqslant 8 E\left[\mathcal{N}_{1}\left(\frac{p_{\min }^{4}}{(512)^{2}(16)} \epsilon^{4}, \mathcal{P},\left(\mathbf{X}_{i}\right)_{i=1}^{n}\right)\right] e^{-n p_{\min }^{8} \epsilon^{8} / C_{18}}
$$

for constant $C_{18}>0$ (not depending on $n$ ). To deal with the term $\pi_{n, 3}(\widetilde{p})$, first note that by (5.33) missing probability does not depend on $Y$ or $p_{1}(\mathbf{X})=p_{0}(\mathbf{X})=p(\mathbf{X})$. Now, one may write

$$
\begin{aligned}
\pi_{n, 3}(\widetilde{p}) & =P\left\{E\left[\left|\widetilde{p}_{1}(\mathbf{X})-p_{1}(\mathbf{X})\right| \mid \mathcal{D}_{n}\right]>\frac{\epsilon}{8}\right\}+P\left\{E\left[\left|\widetilde{p}_{0}(\mathbf{X})-p_{0}(\mathbf{X})\right| \mid \mathcal{D}_{n}\right]>\frac{\epsilon}{8}\right\} \\
& =2 P\left\{E\left[|\widetilde{p}(\mathbf{X})-p(\mathbf{X})| \mid \mathcal{D}_{n}\right]>\frac{\epsilon}{8}\right\} .
\end{aligned}
$$


This term can be bounded as in $\pi_{n, 2}(i i)$, i.e.,

$$
\pi_{n, 3}(\tilde{p}) \leqslant 16 E\left[\mathcal{N}_{1}\left(\frac{\epsilon^{2}}{16(64)}, \mathcal{P},(\mathbf{X})_{i=1}^{n}\right)\right] e^{-n \epsilon^{4} / C_{19}}
$$

This complete the proof of Theorem 5.3.3.

\subsection{Minimizing the Empirical Squared Error in the NMAR Case}

Recall that in Section 2.5, we gave the alternative representation of the optimal classifier:

$$
\psi_{B}(\mathbf{Z}, \delta)=\delta \psi_{1}(\mathbf{Z})+(1-\delta) \psi_{0}(\mathbf{X})
$$

where $\psi_{1}$ and $\psi_{0}$ were

$$
\begin{aligned}
& \psi_{1}(\mathbf{Z})= \begin{cases}1 & \text { if } E(\delta(2 Y-1) \mid \mathbf{Z})>0 \\
0 & \text { otherwise }\end{cases} \\
& \psi_{0}(\mathbf{X})= \begin{cases}1 & \text { if } E((1-\delta)(2 Y-1) \mid \mathbf{X})>0 \\
0 & \text { otherwise }\end{cases}
\end{aligned}
$$

Let $\Gamma$ be a class of functions $\gamma: \mathcal{R}^{p} \rightarrow[-1,1]$. Also, let $\mathcal{I}$ be a class of functions $\iota: \mathcal{R}^{d} \rightarrow[-1,1]$. Put

$$
e_{n, 1}(\gamma)=\frac{1}{n} \sum_{i=1}^{n}\left|\delta_{i}\left(2 Y_{i}-1\right)-\gamma\left(\mathbf{Z}_{i}\right)\right|^{2}
$$

and

$$
e_{n, 0}(\iota)=\frac{1}{n} \sum_{i=1}^{n}\left|\left(1-\delta_{i}\right)\left(2 Y_{i}-1\right)-\iota\left(\mathbf{X}_{i}\right)\right|^{2}
$$


Now assume $\gamma_{n}=\widehat{E}(\delta(2 Y-1) \mid \mathbf{Z})$ and $\iota_{n}=\widehat{E}((1-\delta)(2 Y-1) \mid \mathbf{X})$ are selected by minimizing the empirical squared errors over $\Gamma$ and $\mathcal{I}$ respectively, that is,

$$
\gamma_{n}=\underset{\gamma \in \Gamma}{\operatorname{argmin}} e_{n, 1}(\gamma)
$$

and

$$
\iota_{n}=\underset{\iota \in \mathcal{I}}{\operatorname{argmin}} e_{n, 0}(\iota)
$$

The classifier corresponding to $\gamma_{n}$ and $\iota_{n}$ is define by

$$
\hat{\hat{\psi}}_{n}(\mathbf{Z}, \delta)=\delta \hat{\hat{\psi}}_{n, 1}(\mathbf{Z})+(1-\delta) \hat{\hat{\psi}}_{n, 0}(\mathbf{X})
$$

where $\hat{\hat{\psi}}_{n, 1}(\mathbf{Z})=I\left\{\gamma_{n}(\mathbf{Z})>0\right\}$ and $\hat{\hat{\psi}}_{n, 0}(\mathbf{X})=I\left\{\iota_{n}(\mathbf{X})>0\right\}$. To prove the strong consistency of $\hat{\hat{\psi}}_{n}$, we first state the following theorem. In what follows $L_{n}\left(\hat{\hat{\psi}}_{n}\right)=$ $P\left\{\hat{\hat{\psi}}_{n}(\mathbf{Z}, \delta) \neq Y \mid \mathcal{D}_{n}\right\}$

Theorem 5.4.1 Let $\Gamma$ be a totally bounded class of functions $\gamma: \mathcal{R}^{p} \rightarrow[-1,1]$ containing $E(\delta(2 Y-1) \mid \mathbf{Z})$. Also, let $\mathcal{I}$ be a totally bounded class of functions $\iota: \mathcal{R}^{d} \rightarrow[-1,1]$ containing $E((1-\delta)(2 Y-1) \mid \mathbf{X})$. Then for every $\epsilon>0$ and all $n \geqslant 1$

$$
P\left\{L_{n}\left(\hat{\hat{\psi}}_{n}\right)-L\left(\psi_{B}\right)>\epsilon\right\} \leqslant 4 \Delta_{\infty}\left(\epsilon^{2}, \Gamma, \mathcal{I}\right) e^{-\frac{n \epsilon^{4}}{1152}}
$$

where

$$
\Delta_{\infty}\left(\epsilon^{2}, \Gamma, \mathcal{I}\right)=\mathcal{N}_{\infty}\left(\frac{\epsilon^{2}}{24}, \Gamma\right) \bigvee \mathcal{N}_{\infty}\left(\frac{\epsilon^{2}}{24}, \mathcal{I}\right)
$$




\section{Proof.}

By using Lemma 2.5.2

$$
\begin{aligned}
P\left\{L_{n}\left(\hat{\hat{\psi}}_{n}\right)-L\left(\psi_{B}\right)>\epsilon\right\} \leqslant & P\left\{E\left[\left|\gamma_{n}(\mathbf{Z})-E(\delta(2 Y-1) \mid \mathbf{Z})\right| \mid \mathcal{D}_{n}\right]>\frac{\epsilon}{2}\right\} \\
& +P\left\{E\left[\left|\iota_{n}(\mathbf{X})-E((1-\delta)(2 Y-1) \mid \mathbf{X})\right| \mid \mathcal{D}_{n}\right]>\frac{\epsilon}{2}\right\} .
\end{aligned}
$$

By similar arguments leading to 5.9 in the proof of Theorem 5.2.2, one has

$$
\begin{aligned}
& E\left[\left|\gamma_{n}(\mathbf{Z})-E(\delta(2 Y-1) \mid \mathbf{Z})\right| \mid \mathcal{D}_{n}\right] \\
& \leqslant \sqrt{E\left[\left|\gamma_{n}(\mathbf{Z})-E(\delta(2 Y-1) \mid \mathbf{Z})\right|^{2} \mid \mathcal{D}_{n}\right]} \\
& =\sqrt{E\left[\left|\gamma_{n}(\mathbf{Z})-\delta(2 Y-1)\right|^{2} \mid \mathcal{D}_{n}\right]-E|E(\delta(2 Y-1) \mid \mathbf{Z})-\delta(2 Y-1)|^{2} \mid} \\
& =\sqrt{E\left[\left|\gamma_{n}(\mathbf{Z})-\delta(2 Y-1)\right|^{2} \mid \mathcal{D}_{n}\right]-\inf _{\gamma \in \Gamma} E|\gamma(\mathbf{Z})-\delta(2 Y-1)|^{2} \mid} \\
& :=\sqrt{J(n)} .
\end{aligned}
$$

But

$$
\begin{aligned}
J(n) & =\sup _{\gamma \in \Gamma}\left[E\left[\left|\gamma_{n}(\mathbf{Z})-\delta(2 Y-1)\right|^{2} \mid \mathcal{D}_{n}\right]-e_{n, 1}\left(\gamma_{n}\right)+e_{n, 1}\left(\gamma_{n}\right)-e_{n, 1}(\gamma)+e_{n, 1}(\gamma)\right. \\
& \left.-E|\gamma(\mathbf{Z})-\delta(2 Y-1)|^{2}\right] \\
& \leqslant 2 \sup _{\gamma \in \Gamma}\left|e_{n, 1}(\gamma)-E\right| \delta(2 Y-1)-\left.\gamma(\mathbf{Z})\right|^{2} \mid \\
& =\sup _{\gamma \in \Gamma}\left|\frac{1}{n} \sum_{i=1}^{n}\left(\delta_{i}\left(2 Y_{i}-1\right)-\gamma\left(\mathbf{Z}_{i}\right)\right)^{2}-E(\delta(2 Y-1)-\gamma(\mathbf{Z}))^{2}\right| .
\end{aligned}
$$

Finally, with the same arguments for the second term in right hand side of (5.35), one has 


$$
\begin{aligned}
& P\left\{L_{n}\left(\hat{\hat{\psi}}_{n}\right)-L\left(\psi_{B}\right)>\epsilon\right\} \\
& \leqslant P\left\{\sup _{\gamma \in \Gamma}\left|\frac{1}{n} \sum_{i=1}^{n}\left(\delta_{i}\left(2 Y_{i}-1\right)-\gamma\left(\mathbf{Z}_{i}\right)\right)^{2}-E(\delta(2 Y-1)-\gamma(\mathbf{Z}))^{2}\right|>\frac{\epsilon^{2}}{8}\right\} \\
& +P\left\{\sup _{\iota \in \mathcal{I}} \mid \frac{1}{n} \sum_{i=1}^{n}\left(\left(1-\delta_{i}\right)\left(2 Y_{i}-1\right)-\iota\left(\mathbf{X}_{i}\right)\right)^{2}\right. \\
& \left.-E((1-\delta)(2 Y-1)-\iota(\mathbf{X}))^{2} \mid>\frac{\epsilon^{2}}{8}\right\} .
\end{aligned}
$$

Let $\Gamma_{\frac{\epsilon^{2}}{24}}$ be an $\frac{\epsilon^{2}}{24}$-cover of $\Gamma$ w.r.t $\|\cdot\|_{\infty}$ of minimal cardinality. That is, for every $\gamma \in$ $\Gamma$, there is a $\gamma^{*} \in \Gamma_{\frac{\epsilon^{2}}{24}}$ such that $\left\|\gamma-\gamma^{*}\right\|_{\infty}<\frac{\epsilon^{2}}{24}$. Let $g_{1}(\mathbf{Z}, Y, \delta)=(\delta(2 Y-1)-\gamma(\mathbf{Z}))^{2}$ and $g_{1}^{*}(\mathbf{Z}, Y, \delta)=\left(\delta(2 Y-1)-\gamma^{*}(\mathbf{Z})^{2}\right.$ and observe that

$$
\begin{aligned}
& \left|\frac{1}{n} \sum_{i=1}^{n}\left(\delta_{i}\left(2 Y_{i}-1\right)-\gamma\left(\mathbf{Z}_{i}\right)\right)^{2}-E(\delta(2 Y-1)-\gamma(\mathbf{Z}))^{2}\right| \\
& =\left|\frac{1}{n} \sum_{i=1}^{n} g_{1}\left(\mathbf{Z}_{i}, Y_{i}, \delta_{i}\right)-E\left(g_{1}(\mathbf{Z}, Y, \delta)\right)\right| \\
& \leqslant\left|\frac{1}{n} \sum_{i=1}^{n} g_{1}\left(\mathbf{Z}_{i}, Y_{i}, \delta_{i}\right)-\frac{1}{n} \sum_{i=1}^{n} g_{1}^{*}\left(\mathbf{Z}_{i}, Y_{i}, \delta_{i}\right)\right|+\left|\frac{1}{n} \sum_{i=1}^{n} g_{1}^{*}\left(\mathbf{Z}_{i}, Y_{i}, \delta_{i}\right)-E\left(g_{1}^{*}(\mathbf{Z})\right)\right| \\
& \quad+\left|E\left(g_{1}^{*}(\mathbf{Z})-g_{1}(\mathbf{Z})\right)\right| \\
& \leqslant\left\|g_{1}-g_{1}^{*}\right\|_{\infty}+\left|\frac{1}{n} \sum_{i=1}^{n} g_{1}^{*}\left(\mathbf{Z}_{i}, Y_{i}, \delta_{i}\right)-E\left(g_{1}^{*}(\mathbf{Z})\right)\right|+\left\|g_{1}-g_{1}^{*}\right\|_{\infty} \\
& \leqslant \frac{\epsilon^{2}}{12}+\left|\frac{1}{n} \sum_{i=1}^{n} g_{1}^{*}\left(\mathbf{Z}_{i}, Y_{i}, \delta_{i}\right)-E\left(g_{1}^{*}(\mathbf{Z})\right)\right| .
\end{aligned}
$$


Hence

$$
\begin{aligned}
& P\left\{\sup _{\gamma \in \Gamma}\left|\frac{1}{n} \sum_{i=1}^{n}\left(\delta_{i}\left(2 Y_{i}-1\right)-\gamma\left(\mathbf{Z}_{i}\right)\right)^{2}-E(\delta(2 Y-1)-\gamma(\mathbf{Z}))^{2}\right|>\frac{\epsilon^{2}}{8}\right\} \\
& \leqslant P\left\{\sup _{\gamma \in \Gamma_{\frac{\epsilon^{2}}{24}}}\left|\frac{1}{n} \sum_{i=1}^{n}\left(\delta_{i}\left(2 Y_{i}-1\right)-\gamma\left(\mathbf{Z}_{i}\right)\right)^{2}-E(\delta(2 Y-1)-\gamma(\mathbf{Z}))^{2}\right|>\frac{\epsilon^{2}}{24}\right\} \\
& \leqslant 2 \mathcal{N}_{\infty}\left(\frac{\epsilon^{2}}{24}, \Gamma\right) \sup _{\gamma \in \Gamma_{\frac{\epsilon^{2}}{24}}} P\left\{\mid \frac{1}{n} \sum_{i=1}^{n}\left(\delta_{i}\left(2 Y_{i}-1\right)-\gamma\left(\mathbf{Z}_{i}\right)\right)^{2}\right. \\
& \left.-E(\delta(2 Y-1)-\gamma(\mathbf{Z}))^{2} \mid>\frac{\epsilon^{2}}{24}\right\} \\
& \leqslant 2 \mathcal{N}_{\infty}\left(\frac{\epsilon^{2}}{24}, \Gamma\right) e^{-\frac{n \epsilon^{4}}{1152}} .
\end{aligned}
$$

Similarly, one can show that

$$
\begin{aligned}
& P\left\{\sup _{\iota \in \mathcal{I}} \mid \frac{1}{n} \sum_{i=1}^{n}\left(\left(1-\delta_{i}\right)\left(2 Y_{i}-1\right)-\iota\left(\mathbf{X}_{i}\right)\right)^{2}\right. \\
& \left.-E((1-\delta)(2 Y-1)-\iota(\mathbf{X}))^{2} \mid>\frac{\epsilon^{2}}{8}\right\} \\
& \leqslant 2 \mathcal{N}_{\infty}\left(\frac{\epsilon^{2}}{24}, \mathcal{I}\right) e^{-\frac{n \epsilon^{4}}{1152}}
\end{aligned}
$$

Finally, from (5.36),

$$
P\left\{L_{n}\left(\hat{\hat{\psi}}_{n}\right)-L\left(\psi_{B}\right)>\epsilon\right\} \leqslant 2 \mathcal{N}_{\infty}\left(\frac{\epsilon^{2}}{24}, \Gamma\right) e^{-\frac{n \epsilon^{4}}{1152}}+2 \mathcal{N}_{\infty}\left(\frac{\epsilon^{2}}{24}, \mathcal{I}\right) e^{-\frac{n \epsilon^{4}}{1152}}
$$

This completes the proof of the theorem.

The supremum norm is often too strong a condition for consistency. To motivate a weaker condition, let us again consider the empirical $L_{1}$-norm which results in data-dependent covers. The following result provides upper bounds on $P\left\{L_{n}\left(\hat{\hat{\psi}}_{n}\right)-\right.$ $\left.L\left(\psi_{B}\right)>\epsilon\right\}$ based on empirical $L_{1}$ covering numbers. 
Theorem 5.4.2 For any $n$ and $\epsilon>0$,

$$
P\left\{L_{n}\left(\hat{\hat{\psi}}_{n}\right)-L\left(\psi_{B}\right)>\epsilon\right\} \leqslant 16 \Delta_{1}\left(\epsilon^{2}, \Gamma, \mathcal{I}\right) e^{-\frac{n \epsilon^{4}}{\left(64^{2}\right) 256}},
$$

where

$$
\Delta_{1}\left(\epsilon^{2}, \Gamma, \mathcal{I}\right)=E\left[\mathcal{N}_{1}\left(\frac{\epsilon^{2}}{64}, \Gamma,\left(\mathbf{Z}_{i}, Y_{i}, \delta_{i}\right)_{i=1}^{n}\right) \bigvee \mathcal{N}_{1}\left(\frac{\epsilon^{2}}{64}, \mathcal{I},\left(\mathbf{X}_{i}, Y_{i}, \delta_{i}\right)_{i=1}^{n}\right)\right]
$$

\section{Proof.}

According to (5.36)

$$
\begin{aligned}
& P\left\{L_{n}\left(\hat{\hat{\psi}}_{n}\right)-L\left(\psi_{B}\right)>\epsilon\right\} \\
& \leqslant P\left\{\sup _{\gamma \in \Gamma}\left|\frac{1}{n} \sum_{i=1}^{n}\left(\delta_{i}\left(2 Y_{i}-1\right)-\gamma\left(\mathbf{Z}_{i}\right)\right)^{2}-E(\delta(2 Y-1)-\gamma(\mathbf{Z}))^{2}\right|>\frac{\epsilon^{2}}{8}\right\} \\
& +P\left\{\sup _{\iota \in \mathcal{T}} \mid \frac{1}{n} \sum_{i=1}^{n}\left(\left(1-\delta_{i}\right)\left(2 Y_{i}-1\right)-\iota\left(\mathbf{X}_{i}\right)\right)^{2}\right. \\
& \left.-E((1-\delta)(2 Y-1)-\iota(\mathbf{X}))^{2} \mid>\frac{\epsilon^{2}}{8}\right\} .
\end{aligned}
$$

Now a quick scan through the proof of Theorem 5.3.1 reveals that, by using similar arguments we obtain the following performance bound

$$
\begin{aligned}
P\left\{L_{n}\left(\hat{\psi}_{n}\right)-L\left(\psi_{B}\right)>\epsilon\right\} & \leqslant 8 E\left[\mathcal{N}_{1}\left(\frac{\epsilon^{2}}{64}, \Gamma,\left(\mathbf{Z}_{i}, Y_{i}, \delta_{i}\right)_{i=1}^{n}\right)\right] e^{-\frac{n \epsilon^{4}}{\left(64^{2}\right) 256}} \\
& +8 E\left[\mathcal{N}_{1}\left(\frac{\epsilon^{2}}{64}, \mathcal{I},\left(\mathbf{X}_{i}, Y_{i}, \delta_{i}\right)_{i=1}^{n}\right)\right] e^{-\frac{n \epsilon^{4}}{\left(64^{2}\right) 256}}
\end{aligned}
$$

This completes the proof.

Remark 5.4.3 In this section, we proposed a data-based classifier based on the new representation of the best classifier in (2.22) along with (2.23) and (2.24). A 
serious problem concerning the applicability of the method introduced above is that it requires information about the classes $\Gamma$ and $\mathcal{I}$, and in particular, whether the functions $E(\delta(2 Y-1) \mid \mathbf{Z})$ and $E((1-\delta)(2 Y-1) \mid \mathbf{X})$, are contained in the classes $\Gamma$ and $\mathcal{I}$ respectively. 


\section{Chapter 6}

\section{Simulation Studies}

\subsection{Introduction}

In this chapter, we carried out different simulations to study the finite-sample performance of the proposed methods in the previous chapters. We consider artificial examples along with the real data example. In Chapter 2, we derived the best classifier when a block of covariates was missing and showed that the proposed classifier is always the best classifier theoretically and numerically (see; Theorem 2.2.1 and Example 1 in Chapter 2). The model that we have used for the missing probability mechanism was in an exponential form, but in practice, some statisticians believe that the logistic model is more appropriate. In Section 6.2, we consider the logistic model and study the effects of the missing probability mechanism by using more numerical examples and we consider the different mechanisms (MCAR, MAR, and NMAR) with different degrees of dependency between variables. Also, in Chapters 2 and 4 , the parametric and nonparametric data-based classifiers were proposed when a block of covariates was missing. In Section 6.3, we compare these classifiers by 
running a simulation study and showing that, in the NMAR cases, the nonparametric classifiers perform better than those are parametric. Furthermore, in Chapter 3 , the general Swiss-cheese model was studied. To illustrate the advantage of using this model, we consider a real data set, which contains 4 different missing patterns and show that the nonparametric classifier based on these four missing patterns is superior to other method of classification.

\subsection{Effects of Missing Probability Mechanism}

In this section, we give more numerical examples to illustrate the possible effects of missing covariates on misclassification error rates. In these examples, we study the prediction of the class membership, $Y=0$ or 1, of an entity based on the covariates $\mathbf{Z}=(X, V)^{\prime}$, where $V$ can be missing but not $X$. The bivariate distribution of $\mathbf{Z}$ is the same as those of Example 1 in Chapter 2, i.e., $\mathbf{Z} \sim N_{2}\left(\boldsymbol{\mu}_{0}, \Sigma_{0}\right)$ if $Y=0$ and $\mathbf{Z} \sim N_{2}\left(\boldsymbol{\mu}_{1}, \Sigma_{1}\right)$ otherwise, where

$$
\boldsymbol{\mu}_{0}=\left(\begin{array}{l}
2 \\
2
\end{array}\right), \quad \boldsymbol{\mu}_{1}=\left(\begin{array}{c}
0.5 \\
1
\end{array}\right), \quad \Sigma_{0}=\left(\begin{array}{cc}
1 & \sigma_{x v} \\
\sigma_{x v} & 2.25
\end{array}\right), \quad \Sigma_{1}=\left(\begin{array}{cc}
1 & \sigma_{x v} \\
\sigma_{x v} & 1
\end{array}\right)
$$

Here, we have considered three values for $\sigma_{x v}: 0.15,0.8$, and 0 . The missing data mechanism is taken to be the logistical model

$$
\begin{aligned}
p(\mathbf{z}, y) & =p(x, v, y):=P\{\delta=1 \mid X=x, V=v, Y=y\} \\
& =\frac{1}{1+\exp \left\{-\left[(1-a y)\left(b_{0}+b_{1} x+b_{2} v+b_{3} x^{2}+b_{4} v^{2}+b_{5} v x\right)+c y\right]\right\}},
\end{aligned}
$$

where $a, b_{0}, b_{1}, b_{2}, b_{3}, b_{4}, b_{5}$ and $c$ are constant. Note that, the model in (6.1) is allowed to depend on the variables $x$ and $v$ as well as the interaction between them. Recall 
that the best classifier is given by

$$
\psi_{B}(\mathbf{Z}, \delta)=\delta \psi_{1}(\mathbf{Z})+(1-\delta) \psi_{0}(X)
$$

where

$$
\begin{aligned}
& \psi_{1}(\mathbf{Z})= \begin{cases}1 & \text { if } p_{1}(\mathbf{Z}) f_{1}(\mathbf{Z}) p-p_{0}(\mathbf{Z}) f_{0}(\mathbf{Z})(1-p)>0 \\
0 & \text { otherwise }\end{cases} \\
& \psi_{0}(X)= \begin{cases}1 & \text { if }\left(1-q_{1}(X)\right) g_{1}(X) p-\left(1-q_{0}(X)\right) g_{0}(X)(1-p)>0 \\
0 & \text { otherwise. }\end{cases}
\end{aligned}
$$

We have seen in Section 2.2 that

$$
\begin{aligned}
p_{1}(\mathbf{Z}) & =p(\mathbf{Z}, 1), \quad p_{0}(\mathbf{Z})=p(\mathbf{Z}, 0) \\
f_{k}(\mathbf{Z}) & =\text { density function for } N_{2}\left(\boldsymbol{\mu}_{k}, \Sigma_{k}\right), k=0,1 \\
g_{k}(X) & =\text { marginal density of } X \text { for class } k=0,1 \\
q_{1}(X) & =q(X, 1)=E[p(\mathbf{Z}, 1) \mid X] \\
q_{0}(X) & =q(X, 0)=E[p(\mathbf{Z}, 0) \mid X] \\
p & =P\{Y=1\}=1-P\{Y=0\} .
\end{aligned}
$$

For these numerical examples, we considered different values for $a, b_{1}, b_{2}, b_{3}, b_{4}, b_{5}$ and $c$ in order to have different missing mechanisms. Tables 6.1 through 6.9 are based on $p=P\{Y=1\}=0.5,0.25$, and 0.85 . These tables contain various misclassification probabilities for both (6.2) as well as the naïve classifier

$$
\psi_{\text {nä̀ve }}(\mathbf{Z}, \delta)=\delta \breve{\psi}_{1}(\mathbf{Z})+(1-\delta) \breve{\psi}_{0}(X)
$$

where

$$
\breve{\psi}_{1}(\mathbf{Z})=\left\{\begin{array}{ll}
1 & \text { if } P\{Y=1 \mid \mathbf{Z}\}>\frac{1}{2} \\
0 & \text { otherwise }
\end{array} \breve{\psi}_{0}(X)= \begin{cases}1 & \text { if } P\{Y=1 \mid X\}>\frac{1}{2} \\
0 & \text { otherwise }\end{cases}\right.
$$


Table 6.1: Error rates for $\psi_{B}$ and $\psi_{\text {naive }}$ when $\sigma_{x, v}=0.15$ and $p=0.5$

\begin{tabular}{|c|c|c|c|c|c|c|c|c|c|c|}
\hline$a$ & $b_{0}$ & $b_{1}$ & $b_{2}$ & $b_{3}$ & $b_{4}$ & $b_{5}$ & $c$ & $\begin{array}{c}\text { Error for } \\
\left(\psi_{B}\right)\end{array}$ & $\begin{array}{c}\text { Error for } \\
\left(\psi_{\text {näve }}\right)\end{array}$ & Mechanism \\
\hline- & - & - & - & - & - & - & - & 0.198 & 0.198 & Complete \\
0 & 0 & 0 & 0 & 0 & 0 & 0 & 0 & 0.212 & 0.212 & MCAR \\
0 & 1 & 0.1 & 0 & 0.01 & 0 & 0 & 0 & 0.206 & 0.206 & MAR \\
0.5 & 0 & -1 & 0 & -0.5 & 0 & 0 & 1.2 & 0.173 & 0.213 & MAR \\
0.5 & 0.01 & 0.7 & 0.05 & 0.1 & 0.5 & 0.03 & 0.5 & 0.196 & 0.201 & NMAR \\
1 & 0 & 0 & 0 & -2 & -1.5 & 0 & 0.5 & 0.131 & 0.215 & NMAR \\
1.5 & 0.5 & 0 & -1 & 0 & -1.7 & 0 & 0.5 & 0.108 & 0.221 & NMAR \\
1 & 0 & 0.2 & -1.2 & 0 & 0 & -2 & 1 & 0.126 & 0.216 & NMAR \\
1 & -0.5 & 0.1 & 0.5 & -0.4 & -0.7 & 0.3 & 0.5 & 0.160 & 0.221 & NMAR \\
0.5 & -1 & 0.5 & 1 & 0 & 0 & 0 & -1 & 0.180 & 0.207 & NMAR \\
0 & 0.01 & 0.05 & 0.02 & 0.01 & 0.15 & 0.1 & 0 & 0.205 & 0.207 & NMAR \\
\hline
\end{tabular}


Table 6.2: Error rates for $\psi_{B}$ and $\psi_{\text {naive }}$ when $\sigma_{x v}=0.8$ and $p=0.5$

\begin{tabular}{|c|c|c|c|c|c|c|c|c|c|c|}
\hline$a$ & $b_{0}$ & $b_{1}$ & $b_{2}$ & $b_{3}$ & $b_{4}$ & $b_{5}$ & $c$ & $\begin{array}{c}\text { Error for } \\
\left(\psi_{B}\right)\end{array}$ & $\begin{array}{c}\text { Error for } \\
\left(\psi_{\text {naïve }}\right)\end{array}$ & Mechanism \\
\hline- & - & - & - & - & - & - & - & 0.186 & 0.186 & Complete \\
0 & 0 & 0 & 0 & 0 & 0 & 0 & 0 & 0.206 & 0.206 & MCAR \\
0 & 1 & 0.1 & 0 & 0.01 & 0 & 0 & 0 & 0.197 & 0.197 & MAR \\
0.5 & 0 & -1 & 0 & -0.5 & 0 & 0 & 1.2 & 0.170 & 0.207 & MAR \\
0.5 & 0.01 & 0.7 & 0.05 & 0.1 & 0.5 & 0.03 & 0.5 & 0.194 & 0.196 & NMAR \\
1 & 0 & 0 & 0 & -2 & -1.5 & 0 & 0.5 & 0.130 & 0.206 & NMAR \\
1.5 & 0.5 & 0 & -1 & 0 & -1.7 & 0 & 0.5 & 0.068 & 0.187 & NMAR \\
1 & 0 & 0.2 & -1.2 & 0 & 0 & -2 & 1 & 0.105 & 0.188 & NMAR \\
1 & -0.5 & 0.1 & 0.5 & -0.4 & -0.7 & 0.3 & 0.5 & 0.155 & 0.208 & NMAR \\
0.5 & -1 & 0.5 & 1 & 0 & 0 & 0 & -1 & 0.196 & 0.216 & NMAR \\
0 & 0.01 & 0.05 & 0.02 & 0.01 & 0.15 & 0.1 & 0 & 0.200 & 0.201 & NMAR \\
\hline
\end{tabular}

Here, the entries in Table 6.1 are the exact misclassification probabilities which are computable (via integration), because the full knowledge of the two class distributions, as well as the form of the missing probability mechanism, $p(x, v, y)$, is completely given. Observe that the error rate of the classifier $\psi_{B}$ is always less than, or equal to, that of the naïve classifier, $\psi_{\text {naive }}$. (In fact, according to Theorem 2.2.1, $\psi_{B}$ has the lowest error rate.) The first row of Table 6.1 corresponds to $P\{\delta=1 \mid \mathrm{Z}, Y\}=1$, i.e., there are no missing covariates; in this case the error rates are 0.198. The misclassification errors for $\psi_{\text {naive }}$ are always greater than 0.198 but the error of $\psi_{B}$ can sometimes be less than 0.198 ; see rows 4 to 10 . This is because the best classifier uses the information from the missing probability mechanism as well. The errors of $\psi_{B}$ and $\psi_{\text {nïve }}$ in the second and third rows are equal. The second 
Table 6.3: Error rates for $\psi_{B}$ and $\psi_{\text {naive }}$ when $\sigma_{x v}=0$ and $p=0.5$

\begin{tabular}{|c|c|c|c|c|c|c|c|c|c|c|}
\hline$a$ & $b_{0}$ & $b_{1}$ & $b_{2}$ & $b_{3}$ & $b_{4}$ & $b_{5}$ & $c$ & $\begin{array}{c}\text { Error for } \\
\left(\psi_{B}\right)\end{array}$ & $\begin{array}{c}\text { Error for } \\
\left(\psi_{\text {naïve }}\right)\end{array}$ & Mechanism \\
\hline- & - & - & - & - & - & - & - & 0.190 & 0.190 & Complete \\
0 & 0 & 0 & 0 & 0 & 0 & 0 & 0 & 0.207 & 0.207 & MCAR \\
0 & 1 & 0.1 & 0 & 0.01 & 0 & 0 & 0 & 0.199 & 0.199 & MAR \\
0.5 & 0 & -1 & 0 & -0.5 & 0 & 0 & 1.2 & 0.172 & 0.208 & MAR \\
0.5 & 0.01 & 0.7 & 0.05 & 0.1 & 0.5 & 0.03 & 0.5 & 0.187 & 0.193 & NMAR \\
1 & 0 & 0 & 0 & -2 & -1.5 & 0 & 0.5 & 0.129 & 0.211 & NMAR \\
1.5 & 0.5 & 0 & -1 & 0 & -1.7 & 0 & 0.5 & 0.113 & 0.222 & NMAR \\
1 & 0 & 0.2 & -1.2 & 0 & 0 & -2 & 1 & 0.127 & 0.216 & NMAR \\
1 & -0.5 & 0.1 & 0.5 & -0.4 & -0.7 & 0.3 & 0.5 & 0.160 & 0.218 & NMAR \\
0.5 & -1 & 0.5 & 1 & 0 & 0 & 0 & -1 & 0.172 & 0.202 & NMAR \\
0 & 0.01 & 0.05 & 0.02 & 0.01 & 0.15 & 0.1 & 0 & 0.194 & 0.196 & NMAR \\
\hline
\end{tabular}


Table 6.4: Error rates for $\psi_{B}$ and $\psi_{\text {naive }}$ when $\sigma_{x v}=0.15$ and $p=0.25$

\begin{tabular}{|c|c|c|c|c|c|c|c|c|c|c|}
\hline$a$ & $b_{0}$ & $b_{1}$ & $b_{2}$ & $b_{3}$ & $b_{4}$ & $b_{5}$ & $c$ & $\begin{array}{c}\text { Error for } \\
\left(\psi_{B}\right)\end{array}$ & $\begin{array}{c}\text { Error for } \\
\left(\psi_{\text {naïe }}\right)\end{array}$ & Mechanism \\
\hline- & - & - & - & - & - & - & - & 0.159 & 0.159 & Complete \\
0 & 0 & 0 & 0 & 0 & 0 & 0 & 0 & 0.167 & 0.167 & MCAR \\
0 & 1 & 0.1 & 0 & 0.01 & 0 & 0 & 0 & 0.163 & 0.163 & MAR \\
0.5 & 0 & -1 & 0 & -0.5 & 0 & 0 & 1.2 & 0.130 & 0.162 & MAR \\
0.5 & 0.01 & 0.7 & 0.05 & 0.1 & 0.5 & 0.03 & 0.5 & 0.158 & 0.161 & NMAR \\
1 & 0 & 0 & 0 & -2 & -1.5 & 0 & 0.5 & 0.090 & 0.167 & NMAR \\
1.5 & 0.5 & 0 & -1 & 0 & -1.7 & 0 & 0.5 & 0.080 & 0.169 & NMAR \\
1 & 0 & 0.2 & -1.2 & 0 & 0 & -2 & 1 & 0.091 & 0.165 & NMAR \\
1 & -0.5 & 0.1 & 0.5 & -0.4 & -0.7 & 0.3 & 0.5 & 0.135 & 0.170 & NMAR \\
0.5 & -1 & 0.5 & 1 & 0 & 0 & 0 & -1 & 0.150 & 0.166 & NMAR \\
0 & 0.01 & 0.05 & 0.02 & 0.01 & 0.15 & 0.1 & 0 & 0.162 & 0.163 & NMAR \\
\hline
\end{tabular}


Table 6.5: Error rates for $\psi_{B}$ and $\psi_{\text {naive }}$ when $\sigma_{x v}=0.8$ and $p=0.25$

\begin{tabular}{|c|c|c|c|c|c|c|c|c|c|c|}
\hline$a$ & $b_{0}$ & $b_{1}$ & $b_{2}$ & $b_{3}$ & $b_{4}$ & $b_{5}$ & $c$ & $\begin{array}{c}\text { Error for } \\
\left(\psi_{B}\right)\end{array}$ & $\begin{array}{c}\text { Error for } \\
\left(\psi_{\text {nä̈e }}\right)\end{array}$ & Mechanism \\
\hline- & - & - & - & - & - & - & - & 0.153 & 0.153 & Complete \\
0 & 0 & 0 & 0 & 0 & 0 & 0 & 0 & 0.163 & 0.163 & MCAR \\
0 & 1 & 0.1 & 0 & 0.01 & 0 & 0 & 0 & 0.158 & 0.158 & MAR \\
0.5 & 0 & -1 & 0 & -0.5 & 0 & 0 & 1.2 & 0.125 & 0.156 & MAR \\
0.5 & 0.01 & 0.7 & 0.05 & 0.1 & 0.5 & 0.03 & 0.5 & 0.158 & 0.159 & NMAR \\
1 & 0 & 0 & 0 & -2 & -1.5 & 0 & 0.5 & 0.093 & 0.158 & NMAR \\
1.5 & 0.5 & 0 & -1 & 0 & -1.7 & 0 & 0.5 & 0.058 & 0.154 & NMAR \\
1 & 0 & 0.2 & -1.2 & 0 & 0 & -2 & 1 & 0.077 & 0.153 & NMAR \\
1 & -0.5 & 0.1 & 0.5 & -0.4 & -0.7 & 0.3 & 0.5 & 0.126 & 0.164 & NMAR \\
0.5 & -1 & 0.5 & 1 & 0 & 0 & 0 & -1 & 0.165 & 0.174 & NMAR \\
0 & 0.01 & 0.05 & 0.02 & 0.01 & 0.15 & 0.1 & 0 & 0.162 & 0.162 & NMAR \\
\hline
\end{tabular}


Table 6.6: Error rates for $\psi_{B}$ and $\psi_{\text {naive }}$ when $\sigma_{x v}=0$ and $p=0.25$

\begin{tabular}{|c|c|c|c|c|c|c|c|c|c|c|}
\hline$a$ & $b_{0}$ & $b_{1}$ & $b_{2}$ & $b_{3}$ & $b_{4}$ & $b_{5}$ & $c$ & $\begin{array}{c}\text { Error for } \\
\left(\psi_{B}\right)\end{array}$ & $\begin{array}{c}\text { Error for } \\
\left(\psi_{\text {näive }}\right)\end{array}$ & Mechanism \\
\hline- & - & - & - & - & - & - & - & 0.154 & 0.154 & Complete \\
0 & 0 & 0 & 0 & 0 & 0 & 0 & 0 & 0.164 & 0.164 & MCAR \\
0 & 1 & 0.1 & 0 & 0.01 & 0 & 0 & 0 & 0.159 & 0.159 & MAR \\
0.5 & 0 & -1 & 0 & -0.5 & 0 & 0 & 1.2 & 0.127 & 0.158 & MAR \\
0.5 & 0.01 & 0.7 & 0.05 & 0.1 & 0.5 & 0.03 & 0.5 & 0.153 & 0.156 & NMAR \\
1 & 0 & 0 & 0 & -2 & -1.5 & 0 & 0.5 & 0.090 & 0.163 & NMAR \\
1.5 & 0.5 & 0 & -1 & 0 & -1.7 & 0 & 0.5 & 0.082 & 0.168 & NMAR \\
1 & 0 & 0.2 & -1.2 & 0 & 0 & -2 & 1 & 0.092 & 0.164 & NMAR \\
1 & -0.5 & 0.1 & 0.5 & -0.4 & -0.7 & 0.3 & 0.5 & 0.133 & 0.168 & NMAR \\
0.5 & -1 & 0.5 & 1 & 0 & 0 & 0 & -1 & 0.144 & 0.163 & NMAR \\
0 & 0.01 & 0.05 & 0.02 & 0.01 & 0.15 & 0.1 & 0 & 0.158 & 0.159 & NMAR \\
\hline
\end{tabular}

row corresponds to MCAR and the third row corresponds to the MAR case which does not depend on class membership $Y$ and according to Remark 2.2.4 errors of $\psi_{B}$ and $\psi_{\text {nïve }}$ in these two cases should be equal.

We have also calculated the misclassification error of the classifier based on the $X$-component only (ignoring $V$ and the missing probability mechanism altogether). This was done to see what the numerical gain would be if one kept the variable $V$ in the model. The error in this case turned out to be 0.227 . Interestingly, this error is larger than all other errors in Table 6.1 (as well as Tables 6.2 and 6.3). Table 6.1 also shows the obvious fact that where the missing probability mechanism depends very much on $Y$ (see the coefficients of $Y$ in rows 7 and 8), 
Table 6.7: Error rates for $\psi_{B}$ and $\psi_{\text {naive }}$ when $\sigma_{x v}=0.15$ and $p=0.85$

\begin{tabular}{|c|c|c|c|c|c|c|c|c|c|c|}
\hline$a$ & $b_{0}$ & $b_{1}$ & $b_{2}$ & $b_{3}$ & $b_{4}$ & $b_{5}$ & $c$ & $\begin{array}{c}\text { Error for } \\
\left(\psi_{B}\right)\end{array}$ & $\begin{array}{c}\text { Error for } \\
\left(\psi_{\text {naïre }}\right)\end{array}$ & Mechanism \\
\hline- & - & - & - & - & - & - & - & 0.107 & 0.107 & Complete \\
0 & 0 & 0 & 0 & 0 & 0 & 0 & 0 & 0.114 & 0.114 & MCAR \\
0 & 1 & 0.1 & 0 & 0.01 & 0 & 0 & 0 & 0.111 & 0.111 & MAR \\
0.5 & 0 & -1 & 0 & -0.5 & 0 & 0 & 1.2 & 0.118 & 0.123 & MAR \\
0.5 & 0.01 & 0.7 & 0.05 & 0.1 & 0.5 & 0.03 & 0.5 & 0.105 & 0.107 & NMAR \\
1 & 0 & 0 & 0 & -2 & -1.5 & 0 & 0.5 & 0.094 & 0.122 & NMAR \\
1.5 & 0.5 & 0 & -1 & 0 & -1.7 & 0 & 0.5 & 0.076 & 0.123 & NMAR \\
1 & 0 & 0.2 & -1.2 & 0 & 0 & -2 & 1 & 0.089 & 0.122 & NMAR \\
1 & -0.5 & 0.1 & 0.5 & -0.4 & -0.7 & 0.3 & 0.5 & 0.098 & 0.122 & NMAR \\
0.5 & -1 & 0.5 & 1 & 0 & 0 & 0 & -1 & 0.099 & 0.109 & NMAR \\
0 & 0.01 & 0.05 & 0.02 & 0.01 & 0.15 & 0.1 & 0 & 0.108 & 0.109 & NMAR \\
\hline
\end{tabular}

then the error of $\psi_{B}$ is substantially lower than that with no missing covariates. In Tables 6.2 and 6.3 , we have carried out the same analysis with $\operatorname{cov}(X, V)=0.8$ and $\operatorname{cov}(X, V)=0$ respectively. Tables $6.4,6.5$, and 6.6 are the same as Tables $6.1,6.2$, and 6.3, except that $p=P\{Y=1\}=0.25$. Tables $6.7,6.8$, and 6.9 correspond to $p=P\{Y=1\}=0.85$.

The numerical results of these tables confirm the conclusion of Theorem 2.2.1, i.e., the classifier defined in (6.2) has the lowest error rate. The main message in all 9 tables is the same: Classification with missing covariates can sometimes have a lower misclassification error rate than the case with no missing covariates. 
Table 6.8: Error rates for $\psi_{B}$ and $\psi_{\text {naive }}$ when $\sigma_{x v}=0.8$ and $p=0.85$

\begin{tabular}{|c|c|c|c|c|c|c|c|c|c|c|}
\hline$a$ & $b_{0}$ & $b_{1}$ & $b_{2}$ & $b_{3}$ & $b_{4}$ & $b_{5}$ & $c$ & $\begin{array}{c}\text { Error for } \\
\left(\psi_{B}\right)\end{array}$ & $\begin{array}{c}\text { Error for } \\
\left(\psi_{\text {naïve }}\right)\end{array}$ & Mechanism \\
\hline- & - & - & - & - & - & - & - & 0.099 & 0.099 & Complete \\
0 & 0 & 0 & 0 & 0 & 0 & 0 & 0 & 0.111 & 0.111 & MCAR \\
0 & 1 & 0.1 & 0 & 0.01 & 0 & 0 & 0 & 0.104 & 0.104 & MAR \\
0.5 & 0 & -1 & 0 & -0.5 & 0 & 0 & 1.2 & 0.118 & 0.122 & MAR \\
0.5 & 0.01 & 0.7 & 0.05 & 0.1 & 0.5 & 0.03 & 0.5 & 0.099 & 0.100 & NMAR \\
1 & 0 & 0 & 0 & -2 & -1.5 & 0 & 0.5 & 0.093 & 0.120 & NMAR \\
1.5 & 0.5 & 0 & -1 & 0 & -1.7 & 0 & 0.5 & 0.041 & 0.111 & NMAR \\
1 & 0 & 0.2 & -1.2 & 0 & 0 & -2 & 1 & 0.079 & 0.111 & NMAR \\
1 & -0.5 & 0.1 & 0.5 & -0.4 & -0.7 & 0.3 & 0.5 & 0.096 & 0.118 & NMAR \\
0.5 & -1 & 0.5 & 1 & 0 & 0 & 0 & -1 & 0.101 & 0.109 & NMAR \\
0 & 0.01 & 0.05 & 0.02 & 0.01 & 0.15 & 0.1 & 0 & 0.105 & 0.105 & NMAR \\
\hline
\end{tabular}


Table 6.9: Error rates for $\psi_{B}$ and $\psi_{\text {naïve }}$ when $\sigma_{x v}=0$ and $p=0.85$

\begin{tabular}{|c|c|c|c|c|c|c|c|c|c|c|}
\hline$a$ & $b_{0}$ & $b_{1}$ & $b_{2}$ & $b_{3}$ & $b_{4}$ & $b_{5}$ & $c$ & $\begin{array}{c}\text { Error for } \\
\left(\psi_{B}\right)\end{array}$ & $\begin{array}{c}\text { Error for } \\
\left(\psi_{\text {nä̈e }}\right)\end{array}$ & Mechanism \\
\hline- & - & - & - & - & - & - & - & 0.102 & 0.102 & Complete \\
0 & 0 & 0 & 0 & 0 & 0 & 0 & 0 & 0.112 & 0.112 & MCAR \\
0 & 1 & 0.1 & 0 & 0.01 & 0 & 0 & 0 & 0.106 & 0.106 & MAR \\
0.5 & 0 & -1 & 0 & -0.5 & 0 & 0 & 1.2 & 0.118 & 0.123 & MAR \\
0.5 & 0.01 & 0.7 & 0.05 & 0.1 & 0.5 & 0.03 & 0.5 & 0.100 & 0.102 & NMAR \\
1 & 0 & 0 & 0 & -2 & -1.5 & 0 & 0.5 & 0.094 & 0.121 & NMAR \\
1.5 & 0.5 & 0 & -1 & 0 & -1.7 & 0 & 0.5 & 0.083 & 0.127 & NMAR \\
1 & 0 & 0.2 & -1.2 & 0 & 0 & -2 & 1 & 0.090 & 0.123 & NMAR \\
1 & -0.5 & 0.1 & 0.5 & -0.4 & -0.7 & 0.3 & 0.5 & 0.097 & 0.121 & NMAR \\
0.5 & -1 & 0.5 & 1 & 0 & 0 & 0 & -1 & 0.093 & 0.104 & NMAR \\
0 & 0.01 & 0.05 & 0.02 & 0.01 & 0.15 & 0.1 & 0 & 0.103 & 0.104 & NMAR \\
\hline
\end{tabular}

\subsection{Parametric and Nonparametric Classification For Simulated Data}

The following examples show some advantages of working with nonparametric classifiers (even if the underlying distributions are known, up to a vector of unknown parameters). We compare parametric and nonparametric estimations for the best classifier by using samples of size $n=200$ from two bivariate normals. First note that the plug-in version of $\psi_{B}$, defined in (6.2) for the parametric case, is

$$
\widehat{\psi}_{n}(\mathbf{Z}, \delta)=\delta \psi_{n 1}(\mathbf{Z})+(1-\delta) \psi_{n 0}(X)
$$


where

$$
\begin{aligned}
& \psi_{n 1}(\mathbf{Z})= \begin{cases}1 & \text { if } \widehat{p}_{1}(\mathbf{Z}) \widehat{f}_{1}(\mathbf{Z}) \widehat{p}-\widehat{p}_{0}(\mathbf{Z}) \widehat{f}_{0}(\mathbf{Z})(1-\widehat{p})>0 \\
0 & \text { otherwise }\end{cases} \\
& \psi_{n 0}(X)= \begin{cases}1 & \text { if }\left(1-\widehat{q}_{1}(X)\right) \widehat{g}_{1}(X) \widehat{p}-\left(1-\widehat{q}_{0}(X)\right) \widehat{g}_{0}(X)(1-\widehat{p})>0 \\
0 & \text { otherwise. }\end{cases}
\end{aligned}
$$

Here $\widehat{f}_{1}, \widehat{f}_{0}, \widehat{g}_{1}$, and $\widehat{g}_{0}$ are obtained from the densities $f_{1}, f_{0}, g_{1}, g_{0}$ upon replacing $\mu_{0}, \mu_{1}, \Sigma_{0}$, and $\Sigma_{1}$ by Anderson's MLE estimates (Anderson 1957) $\widehat{\mu_{0}}, \widehat{\mu_{1}}, \widehat{\Sigma}_{0}$, and $\widehat{\Sigma}_{1}$ (see; Section 2.4.1). The quantity $\widehat{p}=\widehat{P(Y=1)}:=\frac{1}{n} \sum_{i=1}^{n} Y_{i}$. Since it is unrealistic to assume that the functional form of the missing mechanism $p_{y}(\mathbf{z})=$ $P\{\delta=1 \mid \mathbf{Z}=\mathbf{z}, y=Y\}$ (as given by (6.1)) is known, one has to estimate $p_{y}(\mathbf{z})$ and $q_{y}(x):=q(x, y), y=0,1$, nonparametrically. In what follows, we shall use a kernel regression method. To make our point, we also impose the popular MAR assumption which may or may not hold, depending on the values of the constants $a, b_{0}, b_{1}, b_{2}, b_{3}, b_{4}, b_{5}$, and $c$ in (6.1). This assumption implies that $p(\mathbf{Z}, Y):=$ $P\{\delta=1 \mid X, V, Y\} \stackrel{\mathrm{MAR}}{=} P\{\delta=1 \mid X, Y\}:=q(X, Y)$. As a result, under the MAR assumption, $p_{k}(\mathbf{Z})=q_{k}(X), k=0,1$, and the kernel regression estimates are

$$
\widehat{q}_{k}(x)=\sum_{i: Y_{i}=k} \delta_{i} \mathcal{J}\left(\frac{X_{i}-x}{h_{n}}\right) / \sum_{i: Y_{i}=k} \mathcal{J}\left(\frac{X_{i}-x}{h_{n}}\right),
$$

where $0 / 0$ is defined to be 0 . This gives

$$
\widehat{q}_{1}(x)=\frac{\sum_{i=1}^{n} Y_{i} \delta_{i} \mathcal{J}\left(\frac{X_{i}-x}{h_{n}}\right)}{\sum_{i=1}^{n} Y_{i} \mathcal{J}\left(\frac{X_{i}-x}{h_{n}}\right)}, \quad \widehat{q}_{0}(x)=\frac{\sum_{i=1}^{n}\left(1-Y_{i}\right) \delta_{i} \mathcal{J}\left(\frac{X_{i}-x}{h_{n}}\right)}{\sum_{i=1}^{n}\left(1-Y_{i}\right) \mathcal{J}\left(\frac{X_{i}-x}{h_{n}}\right)}
$$

The kernel $\mathcal{J}: \mathcal{R} \rightarrow \mathcal{R}$ was taken to be standard Gaussian. Next, for the sample of size $n=200, \widehat{\psi}_{n}$ was constructed and used to classify a testing sequence of 1000 additional observations from the underlying distribution of the data. This entire 
process was repeated a total of 30 times. The results are summarized in Table 6.10. The error rates reported in this table are averages over the 30 Monte Carlo runs. As for our choice of $h$ (for each sample), we used the bootstrap method to find its "optimal" (empirically) value. More specifically, we generated $B=150$ bootstrap samples of size $n=200$ from the original sample and, for each bootstrap sample, we constructed the classifier $\widehat{\psi}_{n}$ for each value of $h$ in the grid $[0.01,0.03, \cdots, 1.21]$. Finally, we computed the error of $\widehat{\psi}_{n}$ committed on an additional sample (bootstrap) of size 1000. The value of $h \in[0.01,0.03, \cdots, 1.21]$ that minimized our average error was our empirically chosen $\hat{h}$. Next, we have also considered the nonparametric kernel classifier $\psi_{n}^{\text {ker }}$

$$
\psi_{n}^{\mathrm{ker}}(\mathbf{Z}, \delta)=\delta \widetilde{g}_{n, 1}(\mathbf{Z})+(1-\delta) \widetilde{g}_{n, 0}(X)
$$

where

$$
\begin{aligned}
& \widetilde{g}_{n, 1}(\mathbf{z})= \begin{cases}1 & \text { if } \sum_{i=1}^{n} \delta_{i}\left(2 Y_{i}-1\right) \mathcal{K}\left(\frac{\mathbf{z}_{i}-\mathbf{z}}{h_{n, \mathbf{z}}}\right)>0 \\
0 & \text { otherwise }\end{cases} \\
& \widetilde{g}_{n, 0}(x)= \begin{cases}1 & \text { if } \sum_{i=1}^{n}\left(1-\delta_{i}\right)\left(2 Y_{i}-1\right) \mathcal{H}\left(\frac{X_{i}-x}{h_{n, x}}\right)>0 \\
0 & \text { otherwise }\end{cases}
\end{aligned}
$$

and $h_{n, \mathbf{z}}$ and $h_{n, x}$ are smoothing parameters of the kernels $\mathcal{K}$ and $\mathcal{H}$ respectively. Once again, the kernel used in (6.6) is standard Gaussian with smoothing parameter $\hat{h}_{\mathbf{z}}$ which is chosen based on the bootstrap approach above. The error rates of this method also appear in Table 6.10. The numbers shown in brackets are the standard errors. These results clearly show, (as anticipated), that the kernel classifier $\psi_{n}^{\text {ker }}$ performs better than the normal-theory classifier $\widehat{\psi}_{n}$ in the NMAR cases. In rows 13, 14 and 15 the missing probability mechanism depends on $X, V$ and $Y$, whereas in rows 16,17 , and 18 it depends on $V$ and $Y$ but not $X$. In other words, the missing 
probability mechanism in rows 16, 17 and 18 are "highly" NMAR in the sense that it does not even depend on $X$ (the observable part of $(X, V)$ ). For the MAR cases, the normal-theory classifier $\widehat{\psi}_{n}$ can be slightly better than the nonparametric classifier $\psi_{n}^{\mathrm{ker}}$.

To demonstrate the advantage of taking the variable $V$ into account, we have also computed the misclassification errors of the classifier constructed based on the $X$ component for each set of generated data, i.e.,

$$
\psi_{n}(x)= \begin{cases}1 & \text { if } \widehat{g}_{1}(X) \widehat{p}-\widehat{g}_{0}(X)(1-\widehat{p})>0 \\ 0 & \text { otherwise }\end{cases}
$$

where $\widehat{g}_{1}$ and $\widehat{g}_{0}$ are obtained from densities $g_{1}$ and $g_{0}$ (defined in (6.3)) upon replacing data-based estimators of means and variances. The results related to this classifier are also reported in Table 6.10. The misclassification error rate for $\psi_{n}(X)$, the classifier based on $X$-component, is higher than the other classifiers in all cases, because it ignores the information in the $V$-componant. In Tables 6.11 and 6.12 , we have repeated the same simulation study for different values of the covariance between $X$ and $V$. Once again, these results confirm our findings in Table 6.10. 
Table 6.10: Error rates for $\widehat{\psi}_{n}$ (MLE) and $\psi_{n}^{\text {ker }}$ (nonparametric) when $\sigma_{x v}=0.15$.

\begin{tabular}{|c|c|c|c|c|c|c|c|c|c|c|c|}
\hline Classifier & $a$ & $b_{0}$ & $b_{1}$ & $b_{2}$ & $b_{3}$ & $b_{4}$ & $b_{5}$ & $c$ & Error & Best & Mechanism \\
\hline$\widehat{\psi}_{n}$ & 0 & 0 & 0 & 0 & 0 & 0 & 0 & 0 & $\begin{array}{c}0.219 \\
(0.013)\end{array}$ & & \\
\hline$\psi_{n}^{\mathrm{ker}}$ & 0 & 0 & 0 & 0 & 0 & 0 & 0 & 0 & $\begin{array}{c}0.226 \\
(0.015)\end{array}$ & 0.212 & MCAR \\
\hline$\psi_{n}(X)$ & 0 & 0 & 0 & 0 & 0 & 0 & 0 & 0 & $\begin{array}{c}0.228 \\
(0.012)\end{array}$ & & \\
\hline$\widehat{\psi}_{n}$ & 0 & 1 & 0.1 & 0 & 0.01 & 0 & 0 & 0 & $\begin{array}{c}0.210 \\
(0.015)\end{array}$ & & \\
\hline$\psi_{n}^{\text {ker }}$ & 0 & 1 & 0.1 & 0 & 0.01 & 0 & 0 & 0 & $\begin{array}{c}0.215 \\
(0.016)\end{array}$ & 0.206 & MAR \\
\hline$\psi_{n}(X)$ & 0 & 1 & 0.1 & 0 & 0.01 & 0 & 0 & 0 & $\begin{array}{c}0.227 \\
(0.012)\end{array}$ & & \\
\hline$\widehat{\psi}_{n}$ & 0.5 & 1 & -1 & 0 & 0 & 0 & 0 & 1.2 & $\begin{array}{c}0.188 \\
(0.013)\end{array}$ & & \\
\hline$\psi_{n}^{\text {ker }}$ & 0.5 & 1 & -1 & 0 & 0 & 0 & 0 & 1.2 & $\begin{array}{c}0.193 \\
(0.014)\end{array}$ & 0.183 & MAR \\
\hline$\psi_{n}(X)$ & 0 & 1 & 0.1 & 0 & 0.01 & 0 & 0 & 0 & $\begin{array}{c}0.229 \\
(0.015)\end{array}$ & & \\
\hline
\end{tabular}

(Continued over)... 
Table 6.10:(Continued)

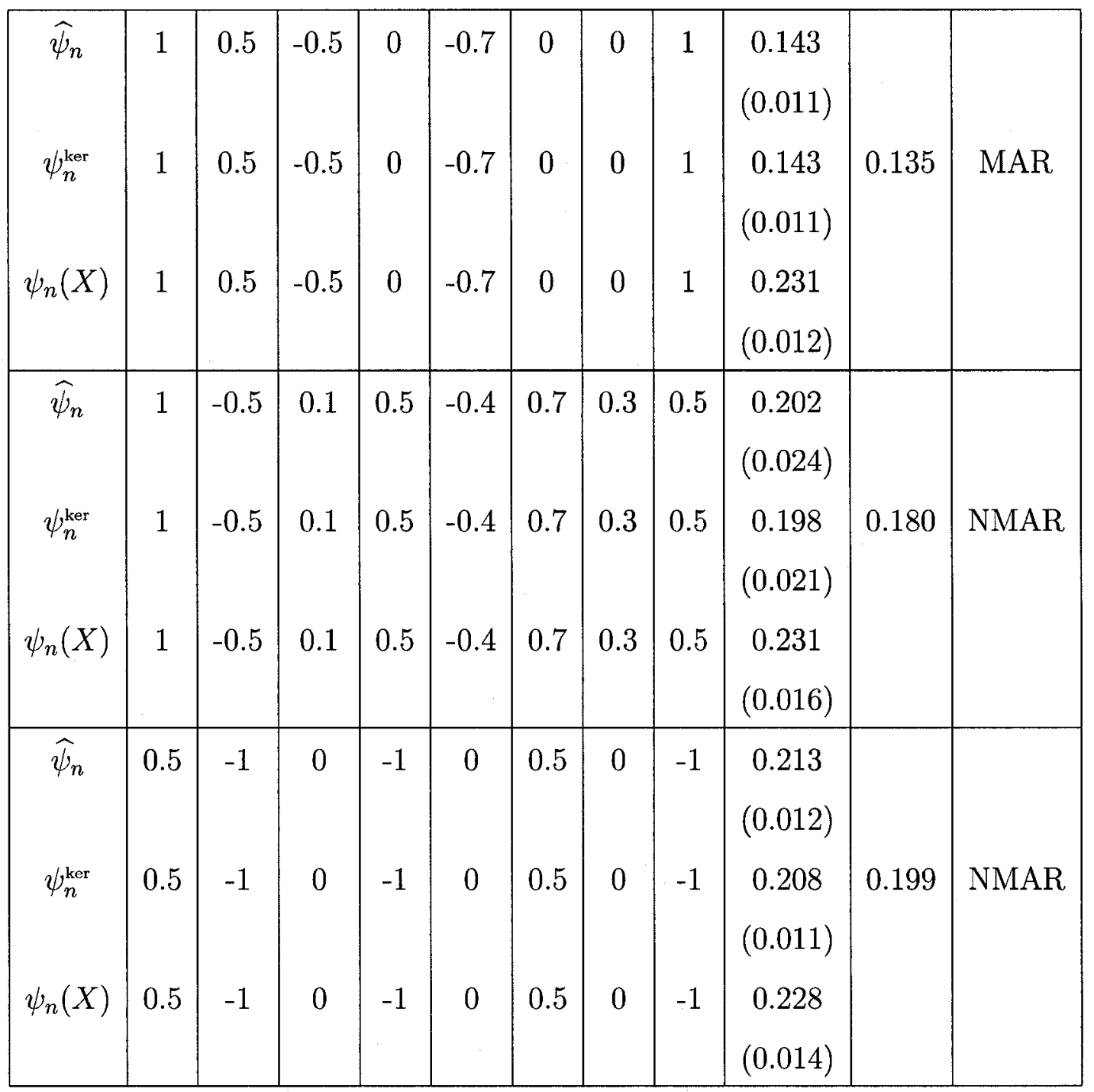


Table 6.11: Error rates for $\widehat{\psi}_{n}$ (MLE) and $\psi_{n}^{\text {ker }}$ (nonparametric) when $\sigma_{x v}=0.8$.

\begin{tabular}{|c|c|c|c|c|c|c|c|c|c|c|c|}
\hline Classifier & $a$ & $b_{0}$ & $b_{1}$ & $b_{2}$ & $b_{3}$ & $b_{4}$ & $b_{5}$ & $c$ & Error & Best & Mechanism \\
\hline$\widehat{\psi}_{n}$ & 0 & 0 & 0 & 0 & 0 & 0 & 0 & 0 & $\begin{array}{c}0.213 \\
(0.012)\end{array}$ & & \\
\hline$\psi_{n}^{\mathrm{ker}}$ & 0 & 0 & 0 & 0 & 0 & 0 & 0 & 0 & $\begin{array}{c}0.223 \\
(0.015)\end{array}$ & 0.206 & MCAR \\
\hline$\psi_{n}(X)$ & 0 & 0 & 0 & 0 & 0 & 0 & 0 & 0 & $\begin{array}{c}0.228 \\
(0.014)\end{array}$ & & \\
\hline$\widehat{\psi}_{n}$ & 0 & 1 & 0.1 & 0 & 0.01 & 0 & 0 & 0 & $\begin{array}{c}0.202 \\
(0.012)\end{array}$ & & \\
\hline$\psi_{n}^{\mathrm{ker}}$ & 0 & 1 & 0.1 & 0 & 0.01 & 0 & 0 & 0 & $\begin{array}{c}0.214 \\
(0.014)\end{array}$ & 0.197 & MAR \\
\hline$\psi_{n}(X)$ & 0 & 1 & 0.1 & 0 & 0.01 & 0 & 0 & 0 & $\begin{array}{c}0.229 \\
(0.010)\end{array}$ & & \\
\hline$\widehat{\psi}_{n}$ & 0.5 & 1 & -1 & 0 & 0 & 0 & 0 & 1.2 & $\begin{array}{c}0.182 \\
(0.012)\end{array}$ & & \\
\hline$\psi_{n}^{\mathrm{ker}}$ & 0.5 & 1 & -1 & 0 & 0 & 0 & 0 & 1.2 & $\begin{array}{c}0.190 \\
(0.014)\end{array}$ & 0.174 & MAR \\
\hline$\psi_{n}(X)$ & 0.5 & 1 & -1 & 0 & 0 & 0 & 0 & 1.2 & $\begin{array}{c}0.229 \\
(0.013)\end{array}$ & & \\
\hline
\end{tabular}

(Continued over)... 
Table 6.11:(Continued)

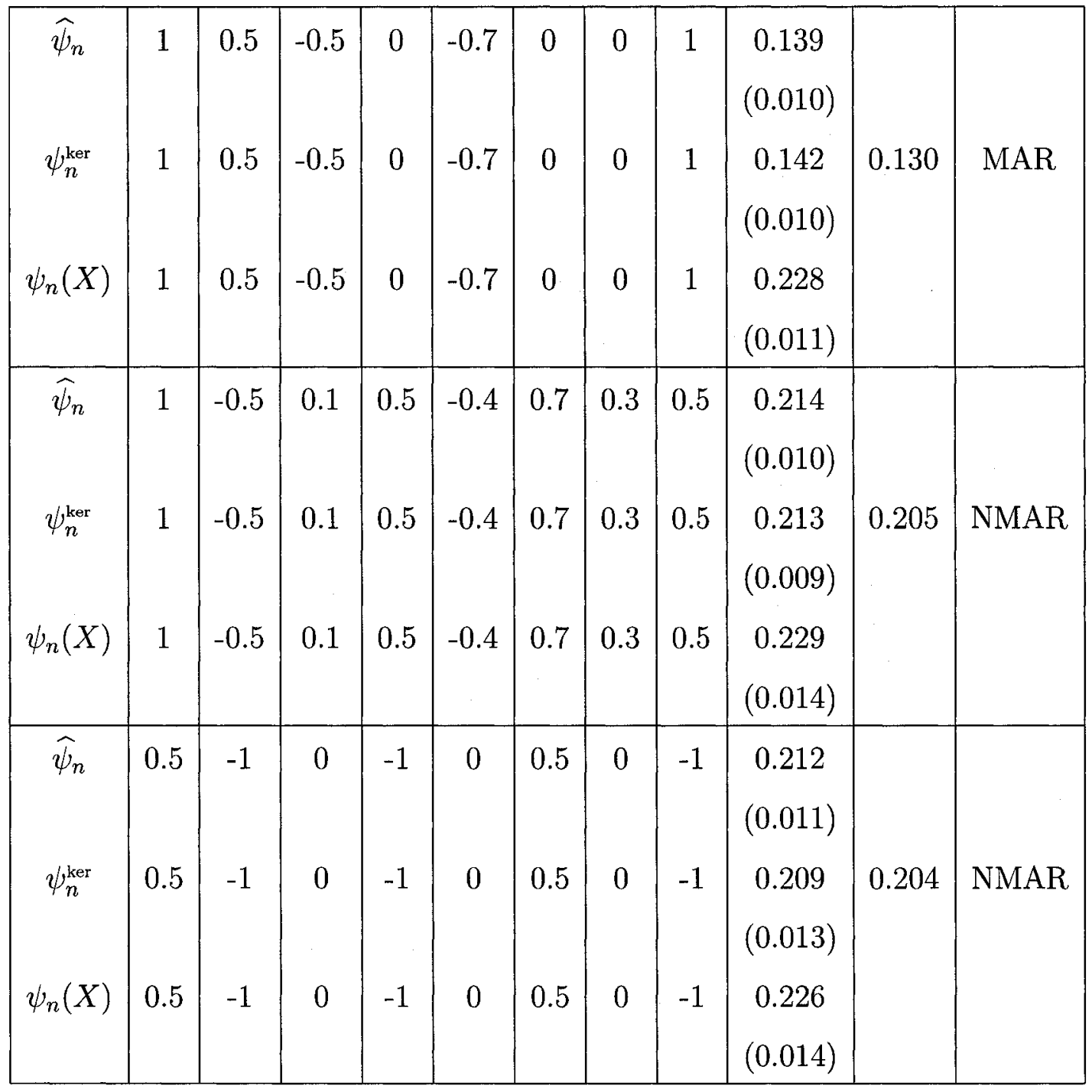


Table 6.12: Error rates for $\widehat{\psi}_{n}$ (MLE) and $\psi_{n}^{\text {ker }}$ (nonparametric) when $\sigma_{x v}=0$.

\begin{tabular}{|c|c|c|c|c|c|c|c|c|c|c|c|}
\hline Classifier & $a$ & $b_{0}$ & $b_{1}$ & $b_{2}$ & $b_{3}$ & $b_{4}$ & $b_{5}$ & $c$ & Error & Best & Mechanism \\
\hline$\widehat{\psi}_{n}$ & 0 & 0 & 0 & 0 & 0 & 0 & 0 & 0 & $\begin{array}{c}0.214 \\
(0.013)\end{array}$ & & \\
\hline$\psi_{n}^{\mathrm{ker}}$ & 0 & 0 & 0 & 0 & 0 & 0 & 0 & 0 & $\begin{array}{c}0.217 \\
(0.015)\end{array}$ & 0.207 & MCAR \\
\hline$\psi_{n}(X)$ & 0 & 0 & 0 & 0 & 0 & 0 & 0 & 0 & $\begin{array}{c}0.233 \\
(0.012)\end{array}$ & & \\
\hline$\widehat{\psi}_{n}$ & 0 & 1 & 0.1 & 0 & 0.01 & 0 & 0 & 0 & $\begin{array}{c}0.204 \\
(0.014)\end{array}$ & & \\
\hline$\psi_{n}^{\mathrm{ker}}$ & 0 & 1 & 0.1 & 0 & 0.01 & 0 & 0 & 0 & $\begin{array}{c}0.210 \\
(0.015)\end{array}$ & 0.199 & MAR \\
\hline$\psi_{n}(X)$ & 0 & 1 & 0.1 & 0 & 0.01 & 0 & 0 & 0 & $\begin{array}{c}0.232 \\
(0.016)\end{array}$ & & \\
\hline$\widehat{\psi}_{n}$ & 0.5 & 1 & -1 & 0 & 0 & 0 & 0 & 1.2 & $\begin{array}{c}0.185 \\
(0.012)\end{array}$ & & \\
\hline$\psi_{n}^{\mathrm{ker}}$ & 0.5 & 1 & -1 & 0 & 0 & 0 & 0 & 1.2 & $\begin{array}{c}0.189 \\
(0.013)\end{array}$ & 0.178 & MAR \\
\hline$\psi_{n}(X)$ & 0.5 & 1 & -1 & 0 & 0 & 0 & 0 & 1.2 & $\begin{array}{c}0.229 \\
(0.013)\end{array}$ & & \\
\hline
\end{tabular}

(Continued over) ... 
Table 6.12:(Continued)

\begin{tabular}{|c|c|c|c|c|c|c|c|c|c|c|l|}
\hline$\widehat{\psi}_{n}$ & 1 & 0.5 & -0.5 & 0 & -0.7 & 0 & 0 & 1 & 0.142 & & \\
$\psi_{n}^{\text {ker }}$ & 1 & 0.5 & -0.5 & 0 & -0.7 & 0 & 0 & 1 & 0.139 & 0.132 & MAR \\
$\psi_{n}(X)$ & 1 & 0.5 & -0.5 & 0 & -0.7 & 0 & 0 & 1 & 0.232 & & \\
\hline$\widehat{\psi}_{n}$ & 1 & -0.5 & 0.1 & 0.5 & -0.4 & 0.7 & 0.3 & 0.5 & 0.185 & & \\
$\psi_{n}^{\text {ker }}$ & 1 & -0.5 & 0.1 & 0.5 & -0.4 & 0.7 & 0.3 & 0.5 & 0.180 & 0.169 & NMAR \\
$\psi_{n}(X)$ & 1 & -0.5 & 0.1 & 0.5 & -0.4 & 0.7 & 0.3 & 0.5 & 0.229 & & \\
\hline$\widehat{\psi}_{n}$ & 0.5 & -1 & 0 & -1 & 0 & 0.5 & 0 & -1 & 0.209 & & \\
$\psi_{n}^{\text {ker }}$ & 0.5 & -1 & 0 & -1 & 0 & 0.5 & 0 & -1 & 0.202 & 0.194 & NMAR \\
$\psi_{n}(X)$ & 0.5 & -1 & 0 & -1 & 0 & 0.5 & 0 & -1 & 0.228 & & \\
& & & & & & & & & $(0.011)$ & & \\
\hline
\end{tabular}


In the next step of our simulation study, we have considered the nonparametric histogram rule introduced in Section 4.2. Recall that

$$
\psi_{n}^{\mathrm{hist}}(\mathbf{Z}, \delta)=\delta \psi_{n, 1}^{\mathrm{hist}}(\mathbf{Z})+(1-\delta) \psi_{n, 0}^{\mathrm{hist}}(\mathbf{X})
$$

where

$$
\begin{aligned}
& \psi_{n, 1}^{\text {hist }}(\mathbf{z})=\left\{\begin{array}{cc}
1 & \text { if } \sum_{i=1}^{n} \delta_{i} Y_{i} I\left\{\mathbf{Z}_{i} \in A_{n}(\mathbf{z})\right\}>\sum_{i=1}^{n} \delta_{i}\left(1-Y_{i}\right) I\left\{\mathbf{Z}_{i} \in A_{n}(\mathbf{z})\right\} \\
0 & \text { otherwise },
\end{array}\right. \\
& \psi_{n, 0}^{\text {hist }}(\mathbf{x})=\left\{\begin{array}{cc}
1 & \text { if } \sum_{i=1}^{n}\left(1-\delta_{i}\right) Y_{i} I\left\{\mathbf{X}_{i} \in B_{n}(\mathbf{x})\right\} \\
0 & >\sum_{i=1}^{n}\left(1-\delta_{i}\right)\left(1-Y_{i}\right) I\left\{\mathbf{X}_{i} \in B_{n}(\mathbf{x})\right\}
\end{array}\right.
\end{aligned}
$$

Here, $A_{n}(\mathbf{z})=A_{n i}$ if $\mathbf{z} \in A_{n i}$, where $\mathbf{z} \in \mathcal{R}^{p}$. Similarly, $B_{n}(\mathbf{x})=B_{n i}$ if $\mathbf{x} \in B_{n i}$, where $\mathbf{x} \in \mathcal{R}^{d}$. We constructed the histogram rules based on the data that we have already generated to construct the normal-theory classifier $\widehat{\psi}_{n}$ and the kernel classifier $\psi_{n}^{\text {ker }}$. Once again, we used the bootstrap method to find the "optimal" (empirically) value of $\ell_{n, \mathbf{z}}$, the size of cubes in $\mathcal{R}^{p}$, in the grid $[0.5,0.53, \cdots, 2.21]$. Finally, we computed the errors of $\psi_{n}^{\text {hist }}$ committed on the future data of size 1000 . The average error rates over 30 Monte Carlo runs appear in Tables 6.13, 6.14, and 6.15. The numbers shown in brackets are the standard errors. To demonstrate the difference between the parametric and the nonparametric classifiers in the different situations, we have reproduced the results of the normal theory classifier, $\widehat{\psi}_{n}$ and the classifier based on $X$-component, $\psi_{n}(X)$, from Tables 6.10, 6.11, and 6.12. Once again, these results clearly show that, the normal theory classifier, $\widehat{\psi}_{n}$ (given by (6.4)) does indeed perform better in all those cases where the MAR assumption holds. On the other hand, when the observations are not MAR, the nonparametric classifiers, $\psi_{n}^{\text {ker }}$ or $\psi_{n}^{\text {hist }}$ perform better than $\widehat{\psi}_{n}$. This is despite the fact that, unlike 
$\widehat{\psi}_{n}$, the nonparametric classifiers do not take advantage of the normality of the underlying populations.

These examples signify the point that in classification with missing covariates, the full knowledge of the underlying distributions alone may not be sufficient to warrant the use of parametric classifiers (such as $\widehat{\psi}_{n}$ ), unless an MAR-type assumption holds. It is important to point out that the classifiers $\psi_{n}^{\mathrm{ker}}$ and $\psi_{n}^{\text {hist }}$ are nonparametric in a stronger sense: They do not depend on the underlying distribution of $(\mathrm{Z}, Y)$, and they do not impose any MAR-type assumption. 
Table 6.13: Error rates for $\widehat{\psi}_{n}$ (MLE) and $\psi_{n}^{\text {hist }}$ (nonparametric) when $\sigma_{x v}=0.15$.

\begin{tabular}{|c|c|c|c|c|c|c|c|c|c|c|c|}
\hline Classifier & $a$ & $b_{0}$ & $b_{1}$ & $b_{2}$ & $b_{3}$ & $b_{4}$ & $b_{5}$ & $c$ & Error & Best & Mechanism \\
\hline$\widehat{\psi}_{n}$ & 0 & 0 & 0 & 0 & 0 & 0 & 0 & 0 & $\begin{array}{c}0.219 \\
(0.013)\end{array}$ & & \\
\hline$\psi_{n}^{\text {hist }}$ & 0 & 0 & 0 & 0 & 0 & 0 & 0 & 0 & $\begin{array}{c}0.226 \\
(0.012)\end{array}$ & 0.212 & MCAR \\
\hline$\psi_{n}(X)$ & 0 & 0 & 0 & 0 & 0 & 0 & 0 & 0 & $\begin{array}{c}0.228 \\
(0.012)\end{array}$ & & \\
\hline$\widehat{\psi}_{n}$ & 0 & 1 & 0.1 & 0 & 0.01 & 0 & 0 & 0 & $\begin{array}{c}0.210 \\
(0.015)\end{array}$ & & \\
\hline$\psi_{n}^{\text {hist }}$ & 0 & 1 & 0.1 & 0 & 0.01 & 0 & 0 & 0 & $\begin{array}{c}0.220 \\
(0.016)\end{array}$ & 0.206 & MAR \\
\hline$\psi_{n}(X)$ & 0 & 1 & 0.1 & 0 & 0.01 & 0 & 0 & 0 & $\begin{array}{c}0.227 \\
(0.012)\end{array}$ & & \\
\hline$\widehat{\psi}_{n}$ & 0.5 & 1 & -1 & 0 & 0 & 0 & 0 & 1.2 & $\begin{array}{c}0.188 \\
(0.013)\end{array}$ & & \\
\hline$\psi_{n}^{\text {hist }}$ & 0.5 & 1 & -1 & 0 & 0 & 0 & 0 & 1.2 & $\begin{array}{c}0.205 \\
(0.017)\end{array}$ & 0.183 & MAR \\
\hline$\psi_{n}(X)$ & 0.5 & 1 & -1 & 0 & 0 & 0 & 0 & 1.2 & $\begin{array}{c}0.229 \\
(0.015)\end{array}$ & & \\
\hline
\end{tabular}

(Continued over)... 
Table 6.13:(Continued)

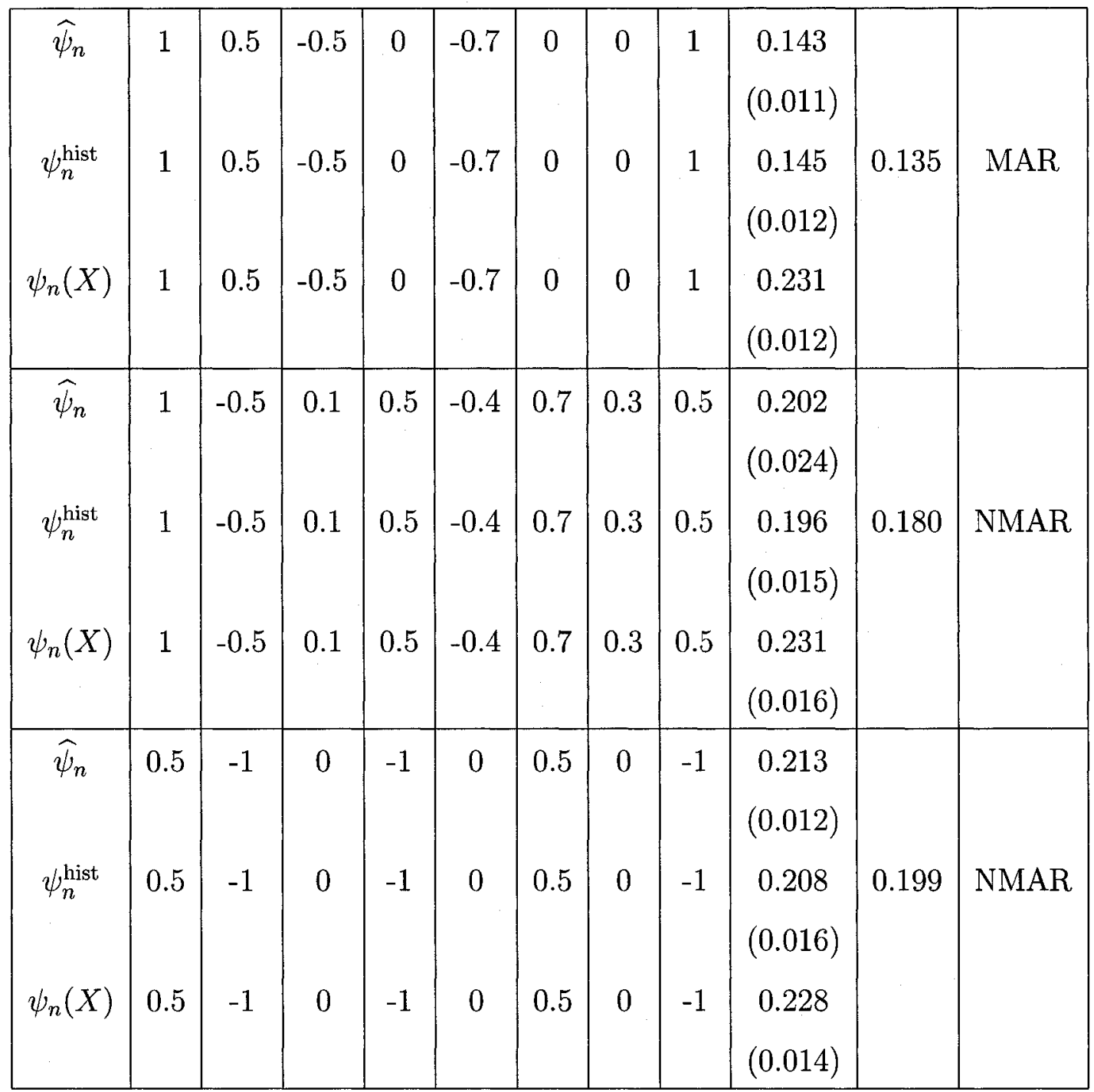


Table 6.14: Error rates for $\widehat{\psi}_{n}$ (MLE) and $\psi_{n}^{\text {hist }}$ (nonparametric) when $\sigma_{x v}=0.8$.

\begin{tabular}{|c|c|c|c|c|c|c|c|c|c|c|c|}
\hline Classifier & $a$ & $b_{0}$ & $b_{1}$ & $b_{2}$ & $b_{3}$ & $b_{4}$ & $b_{5}$ & $c$ & Error & Best & Mechanism \\
\hline$\widehat{\psi}_{n}$ & 0 & 0 & 0 & 0 & 0 & 0 & 0 & 0 & $\begin{array}{c}0.213 \\
(0.012)\end{array}$ & & \\
\hline$\psi_{n}^{\text {hist }}$ & 0 & 0 & 0 & 0 & 0 & 0 & 0 & 0 & $\begin{array}{c}0.223 \\
(0.011)\end{array}$ & 0.206 & MCAR \\
\hline$\psi_{n}(X)$ & 0 & 0 & 0 & 0 & 0 & 0 & 0 & 0 & $\begin{array}{c}0.228 \\
(0.014)\end{array}$ & & \\
\hline$\widehat{\psi}_{n}$ & 0 & 1 & 0.1 & 0 & 0.01 & 0 & 0 & 0 & $\begin{array}{c}0.202 \\
(0.012)\end{array}$ & & \\
\hline$\psi_{n}^{\text {hist }}$ & 0 & 1 & 0.1 & 0 & 0.01 & 0 & 0 & 0 & $\begin{array}{c}0.220 \\
(0.016)\end{array}$ & 0.197 & MAR \\
\hline$\psi_{n}(X)$ & 0 & 1 & 0.1 & 0 & 0.01 & 0 & 0 & 0 & $\begin{array}{c}0.229 \\
(0.010)\end{array}$ & & \\
\hline$\widehat{\psi}_{n}$ & 0.5 & 1 & -1 & 0 & 0 & 0 & 0 & 1.2 & $\begin{array}{c}0.182 \\
(0.012)\end{array}$ & & \\
\hline$\psi_{n}^{\text {hist }}$ & 0.5 & 1 & -1 & 0 & 0 & 0 & 0 & 1.2 & $\begin{array}{c}0.199 \\
(0.012)\end{array}$ & 0.174 & MAR \\
\hline$\psi_{n}(X)$ & 0.5 & 1 & -1 & 0 & 0 & 0 & 0 & 1.2 & $\begin{array}{c}0.229 \\
(0.013)\end{array}$ & & \\
\hline
\end{tabular}

(Continued over)... 
Table 6.14:(Continued)

\begin{tabular}{|c|c|c|c|c|c|c|c|c|c|c|c|}
\hline$\widehat{\psi}_{n}$ & 1 & 0.5 & -0.5 & 0 & -0.7 & 0 & 0 & 1 & $\begin{array}{c}0.139 \\
(0.010)\end{array}$ & & \\
\hline$\psi_{n}^{\text {hist }}$ & 1 & 0.5 & -0.5 & 0 & -0.7 & 0 & 0 & 1 & $\begin{array}{c}0.149 \\
(0.014)\end{array}$ & 0.130 & MAR \\
\hline$\psi_{n}(X)$ & 1 & 0.5 & -0.5 & 0 & -0.7 & 0 & 0 & 1 & $\begin{array}{c}0.228 \\
(0.011)\end{array}$ & & \\
\hline$\widehat{\psi}_{n}$ & 1 & -0.5 & 0.1 & 0.5 & -0.4 & 0.7 & 0.3 & 0.5 & $\begin{array}{c}0.214 \\
(0.010)\end{array}$ & & \\
\hline$\psi_{n}^{\text {hist }}$ & 1 & -0.5 & 0.1 & 0.5 & -0.4 & 0.7 & 0.3 & 0.5 & $\begin{array}{c}0.212 \\
(0.013)\end{array}$ & 0.205 & NMAR \\
\hline$\psi_{n}(X)$ & 1 & -0.5 & 0.1 & 0.5 & -0.4 & 0.7 & 0.3 & 0.5 & $\begin{array}{c}0.229 \\
(0.014)\end{array}$ & & \\
\hline$\widehat{\psi}_{n}$ & 0.5 & -1 & 0 & -1 & 0 & 0.5 & 0 & -1 & $\begin{array}{c}0.212 \\
(0.011)\end{array}$ & & \\
\hline$\psi_{n}^{\text {hist }}$ & 0.5 & -1 & 0 & -1 & 0 & 0.5 & 0 & -1 & $\begin{array}{c}0.210 \\
(0.016)\end{array}$ & 0.204 & NMAR \\
\hline$\psi_{n}(X)$ & 0.5 & -1 & 0 & -1 & 0 & 0.5 & 0 & -1 & $\begin{array}{c}0.226 \\
(0.014)\end{array}$ & & \\
\hline
\end{tabular}


Table 6.15: Error rates for $\widehat{\psi}_{n}$ (MLE) and $\psi_{n}^{\text {hist }}$ (nonparametric) when $\sigma_{x v}=0$.

\begin{tabular}{|c|c|c|c|c|c|c|c|c|c|c|c|}
\hline Classifier & $a$ & $b_{0}$ & $b_{1}$ & $b_{2}$ & $b_{3}$ & $b_{4}$ & $b_{5}$ & $c$ & Error & Best & Mechanism \\
\hline$\widehat{\psi}_{n}$ & 0 & 0 & 0 & 0 & 0 & 0 & 0 & 0 & $\begin{array}{c}0.214 \\
(0.013)\end{array}$ & & \\
\hline$\psi_{n}^{\text {hist }}$ & 0 & 0 & 0 & 0 & 0 & 0 & 0 & 0 & $\begin{array}{c}0.216 \\
(0.011)\end{array}$ & 0.207 & MCAR \\
\hline$\psi_{n}(X)$ & 0 & 0 & 0 & 0 & 0 & 0 & 0 & 0 & $\begin{array}{c}0.233 \\
(0.012)\end{array}$ & & \\
\hline$\widehat{\psi}_{n}$ & 0 & 1 & 0.1 & 0 & 0.01 & 0 & 0 & 0 & $\begin{array}{c}0.204 \\
(0.014)\end{array}$ & & \\
\hline$\psi_{n}^{\text {hist }}$ & 0 & 1 & 0.1 & 0 & 0.01 & 0 & 0 & 0 & $\begin{array}{c}0.214 \\
(0.016)\end{array}$ & 0.199 & MAR \\
\hline$\psi_{n}(X)$ & 0 & 1 & 0.1 & 0 & 0.01 & 0 & 0 & 0 & $\begin{array}{c}0.232 \\
(0.016)\end{array}$ & & \\
\hline$\widehat{\psi}_{n}$ & 0.5 & 1 & -1 & 0 & 0 & 0 & 0 & 1.2 & $\begin{array}{c}0.185 \\
(0.012)\end{array}$ & & \\
\hline$\psi_{n}^{\text {hist }}$ & 0.5 & 1 & -1 & 0 & 0 & 0 & 0 & 1.2 & $\begin{array}{c}0.193 \\
(0.012)\end{array}$ & 0.178 & MAR \\
\hline$\psi_{n}(X)$ & 0.5 & 1 & -1 & 0 & 0 & 0 & 0 & 1.2 & $\begin{array}{c}0.229 \\
(0.013)\end{array}$ & & \\
\hline
\end{tabular}

(Continued over)... 
Table 6.15:(Continued)

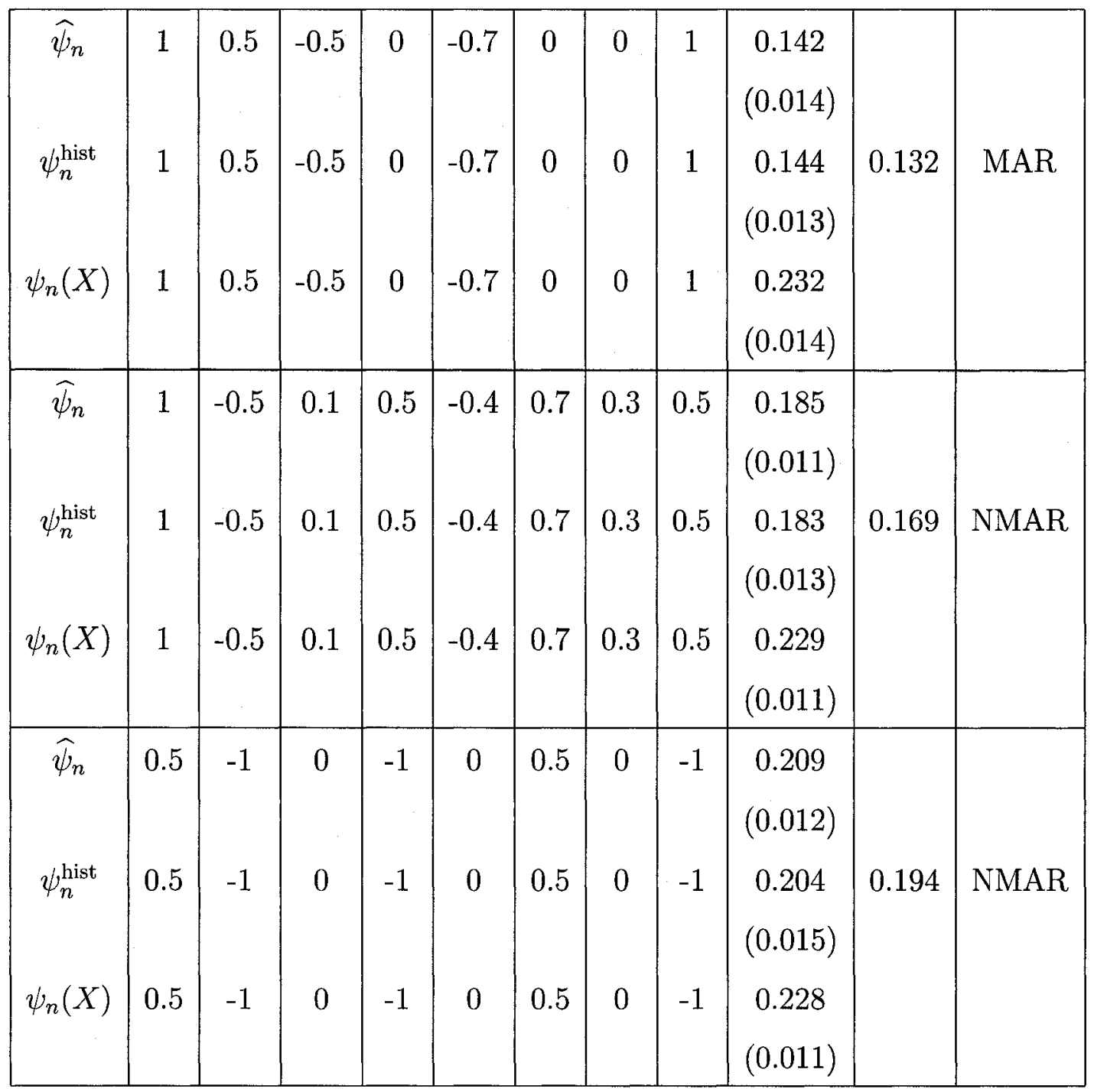

\subsection{A Real Data Set}

This real data set consists of 768 patients, 268 of whom have "tested positive for diabetes", class 1 , and the remaining 500 patients are in class 0 . There are eight numeric-valued covariates associated with each patient. A subset of this data set 
appears in Table 6.16. A full description of this data set is available from the UCI Repository of machine learning databases at $h t t p: / / w w w . i c s . u c i . e d u / \sim m l e a r n$ /MLRepository.html. It is also reported in this website that there are no missing values in this real data set; however, a closer examination of the actual raw data shows that for many patients, the variable $z_{4}=$ 'Triceps skin fold thickness' is reported to be zero. This unusual feature of the data is also observed for the variables $z_{5}=$ '2-Hour serum insulin' and $z_{3}=$ 'Diastolic blood pressure'. In fact, there seems to be three distinct patterns among the covariates: For 27 patients $z_{3}=z_{4}=z_{5}=0$, for 191 patients $z_{4}=z_{5}=0,\left(z_{3} \neq 0\right)$, for 143 patients $z_{5}=0,\left(z_{3} \neq 0, z_{4} \neq 0\right)$. Therefore, to classify a patient's status, it was decided to consider two approaches. One based on the claim that there are no missing values, and a second approach based on treating these zeros (for the variables $z_{3}, z_{4}$, or $z_{5}$ ) as missing values. For the second approach, the 4 "missing patterns" (including the complete cases) correspond to:

$$
\begin{array}{ll}
\mathbf{d}_{1}=(1,1,1,1,1,1,1,1)^{\prime} & \text { a complete case } \\
\mathbf{d}_{2}=(1,1,1,1,0,1,1,1)^{\prime} & z_{5} \text { is missing only } \\
\mathbf{d}_{3}=(1,1,1,0,0,1,1,1)^{\prime} & z_{4}, z_{5} \text { are missing only } \\
\mathbf{d}_{4}=(1,1,0,0,0,1,1,1)^{\prime} & z_{3}, z_{4}, z_{5} \text { are missing only. }
\end{array}
$$

Next, to assess and compare the performance of the two approaches/classifiers, the entire data set was split into a training sample of size $n^{\prime}=568$ (to be used to construct the classifiers) and a testing sequence of size $n-n^{\prime}=200$. To avoid any distributional assumptions on the data, we propose to use non-parametric methods. In particular, it was decided to use kernel classifiers. When the data are treated 
Table 6.16: A portion of the data set

\begin{tabular}{|c|c|c|c|c|c|c|c|c|}
\hline Class & $Z_{1}$ & $Z_{2}$ & $Z_{3}$ & $Z_{4}$ & $Z_{5}$ & $Z_{6}$ & $Z_{7}$ & $Z_{8}$ \\
\hline. &. &. &. &. &. &. &. &. \\
. &. &. &. &. &. &. &. &. \\
0 & 3 & 191 & 68 & 15 & 130 & 30.9 & 0.299 & 34 \\
1 & 3 & 141 & 0 & 0 & 0 & 30.0 & 0.761 & 27 \\
0 & 4 & 95 & 70 & 32 & 0 & 32.1 & 0.612 & 24 \\
0 & 3 & 142 & 80 & 15 & 0 & 32.4 & 0.200 & 63 \\
1 & 4 & 123 & 62 & 0 & 0 & 32.0 & 0.226 & 35 \\
0 & 5 & 96 & 74 & 18 & 67 & 33.6 & 0.997 & 43 \\
1 & 0 & 138 & 0 & 0 & 0 & 36.3 & 0.933 & 25 \\
0 & 2 & 128 & 64 & 42 & 0 & 40.0 & 1.101 & 24 \\
0 & 0 & 102 & 52 & 0 & 0 & 25.1 & 0.078 & 21 \\
1 & 2 & 146 & 0 & 0 & 0 & 27.5 & 0.240 & 28 \\
1 & 10 & 101 & 86 & 37 & 0 & 45.6 & 1.136 & 38 \\
0 & 2 & 108 & 62 & 32 & 56 & 25.2 & 0.128 & 21 \\
0 & 3 & 122 & 78 & 0 & 0 & 23.0 & 0.254 & 40 \\
0 & 1 & 71 & 78 & 50 & 45 & 33.2 & 0.422 & 21 \\
0 & 13 & 106 & 70 & 0 & 0 & 34.2 & 0.251 & 52 \\
0 & 2 & 100 & 70 & 52 & 57 & 40.5 & 0.677 & 25 \\
1 & 7 & 106 & 60 & 24 & 0 & 26.5 & 0.296 & 29 \\
0 & 0 & 104 & 64 & 23 & 116 & 27.8 & 0.454 & 23 \\
0 & 5 & 114 & 74 & 0 & 0 & 24.9 & 0.744 & 57 \\
0 & 2 & 108 & 62 & 10 & 278 & 25.3 & 0.881 & 22 \\
1 & 0 & 146 & 70 & 0 & 0 & 37.9 & 0.334 & 28 \\
0 & 10 & 129 & 76 & 28 & 122 & 35.9 & 0.280 & 39 \\
0 & 7 & 133 & 88 & 15 & 155 & 32.4 & 0.262 & 37 \\
1 & 7 & 161 & 86 & 0 & 0 & 30.4 & 0.165 & 47 \\
. &. &. &. &. &. &. &. &. \\
\hline
\end{tabular}

163 
Table 6.17: Description of variables

\begin{tabular}{|c|c|}
\hline Variable & Description \\
\hline$Z_{1}$ & Number of times pregnant \\
$Z_{2}$ & Plasma glucose concentration a 2 hours in an oral glucose tolerance test \\
$Z_{3}$ & Diastolic blood pressure $(\mathrm{mm} \mathrm{Hg})$ \\
$Z_{4}$ & Triceps skin fold thickness $(\mathrm{mm})$ \\
$Z_{5}$ & 2-Hour serum insulin $(\mathrm{mu} \mathrm{U} / \mathrm{ml})$ \\
$Z_{6}$ & Body mass index (weight in $\left.\mathrm{kg} /(\text { height in } \mathrm{m})^{2}\right)$ \\
$Z_{7}$ & Diabetes pedigree function \\
$Z_{8}$ & Age (years) \\
\hline
\end{tabular}

with missing values, these are the classifiers of the form $\psi_{n}^{\text {ker }}$ given by

$$
\psi_{n}^{\mathrm{ker}}(\mathbf{Z}, \boldsymbol{\delta})=\sum_{k=1}^{4} I\left\{\boldsymbol{\delta}=\mathbf{d}_{k}\right\} \widetilde{\psi}_{n, k}\left(\mathbf{Z}^{(k)}\right)
$$

where

$$
\widetilde{\psi}_{n, k}\left(\mathbf{z}^{(k)}\right)=\left\{\begin{array}{cc}
1 & \text { if } \sum_{i=1}^{n} I\left\{\boldsymbol{\delta}_{i}=\mathbf{d}_{k}\right\} Y_{i} \mathcal{K}_{k}\left(\frac{\mathbf{z}_{i}^{(k)}-\mathbf{z}^{(k)}}{h_{n, k}}\right) \\
& >\sum_{i=1}^{n} I\left\{\boldsymbol{\delta}_{i}=\mathbf{d}_{k}\right\}\left(1-Y_{i}\right) \mathcal{K}_{k}\left(\frac{\mathbf{z}_{i}^{(k)}-\mathbf{z}^{(k)}}{h_{n, k}}\right) \\
0 & \text { otherwise. }
\end{array}\right.
$$

Here $\mathcal{K}_{k}: \mathcal{R}^{p(k)} \rightarrow \mathcal{R}$ is the $k^{\text {th }}$ kernel, where $p(k)=\#$ of 1 's in $\mathbf{d}_{k}$. As for the choice of the kernels, standard Gaussian kernels were used for all cases. The results are summarized in Table 6.18 .

We have also included two other classifiers: The usual kernel classifier $\widetilde{\psi}_{n}^{\text {ker }}$ corresponding to no missing values and the kernel classifier $\bar{\psi}_{n}^{\text {ker }}$ that takes into account only those covariates which are always observable. Table 6.18 gives the average error rates, committed on the testing sequence, over 100 such sample splits. The empirical choice of the smoothing parameters $\hat{h}_{1}, \hat{h}_{2}, \hat{h}_{3}$, and $\hat{h}_{4}$ is based on the 
Table 6.18: Error rates for $\psi_{n}^{\text {ker }}, \widetilde{\psi}_{n}^{\text {ker }}$, and $\bar{\psi}_{n}^{\text {ker }}$, when $n=564$

\begin{tabular}{|c|c|c|c|}
\hline Classifier & $\psi_{n}^{\text {ker }}$ & $\widetilde{\psi}_{n}^{\text {ker }}$ & $\bar{\psi}_{n}^{\text {ker }}$ \\
\hline \multirow[t]{2}{*}{ Error } & 0.259 & 0.322 & 0.352 \\
\hline & $(0.029)$ & $(0.027)$ & $(0.030)$ \\
\hline
\end{tabular}

Table 6.19: Error rates for $\psi_{n}^{\mathrm{ker}}, \widetilde{\psi}_{n}^{\mathrm{ker}}$, and $\bar{\psi}_{n}^{\mathrm{ker}}$, when $n=400$

\begin{tabular}{|c|c|c|c|}
\hline Classifier & $\psi_{n}^{\text {ker }}$ & $\widetilde{\psi}_{n}^{\text {ker }}$ & $\bar{\psi}_{n}^{\text {ker }}$ \\
\hline \multirow[t]{2}{*}{ Error } & 0.267 & 0.325 & 0.354 \\
\hline & $(0.026)$ & $(0.028)$ & $(0.033)$ \\
\hline
\end{tabular}

Table 6.20: Error rates for $\psi_{n}^{\mathrm{ker}}, \widetilde{\psi}_{n}^{\mathrm{ker}}$, and $\bar{\psi}_{n}^{\mathrm{ker}}$, when $n=200$

\begin{tabular}{|c|c|c|c|}
\hline Classifier & $\psi_{n}^{\text {ker }}$ & $\widetilde{\psi}_{n}^{\text {ker }}$ & $\bar{\psi}_{n}^{\text {ker }}$ \\
\hline Error & $\begin{array}{c}0.282 \\
(0.028)\end{array}$ & $\begin{array}{c}0.326 \\
(0.030)\end{array}$ & $\begin{array}{c}0.357 \\
(0.026)\end{array}$ \\
\hline
\end{tabular}


method explained in Section 6.3. Table 6.18 clearly shows that $\psi_{n}^{\mathrm{ker}}$ is far superior to both $\widetilde{\psi}_{n}^{\text {ker }}$ and $\bar{\psi}_{n}^{\text {ker }}$. In fact more is true. Figure 6.1 shows the plot of $h$ versus the

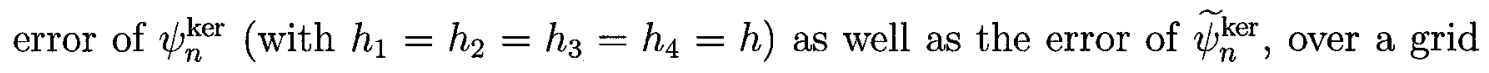
of 50 equally spaced values of $h$ in $(0.01,1.5)$. This plot confirms that even in the sub-optimal case where we have chosen $h_{1}=h_{2}=h_{3}=h_{4}=h$ (for the ease of computation), the classifier $\psi_{n}^{\mathrm{ker}}$, that treats the zeros as missing values, still uniformly (over $h$ ) outperforms the classifier $\widetilde{\psi}_{n}^{\text {ker }}$. We have also repeated this example with sample splits of $n=400$ and $n=200$, (and the same testing sequence of size 200 as before). The results are summarized in Tables 6.19 and 6.20 as well as Figures 6.2 and 6.3. Once again these results verify that $\psi_{n}^{\text {ker }}$ is superior to both $\widetilde{\psi}_{n}^{\text {ker }}$ and $\bar{\psi}_{n}^{\text {ker }}$. 
Figure 6.1: Plot of $h$ versus the error, o represents $\psi_{n}^{\text {ker }}, \bullet$ represents $\widetilde{\psi}_{n}^{\text {ker }}$.

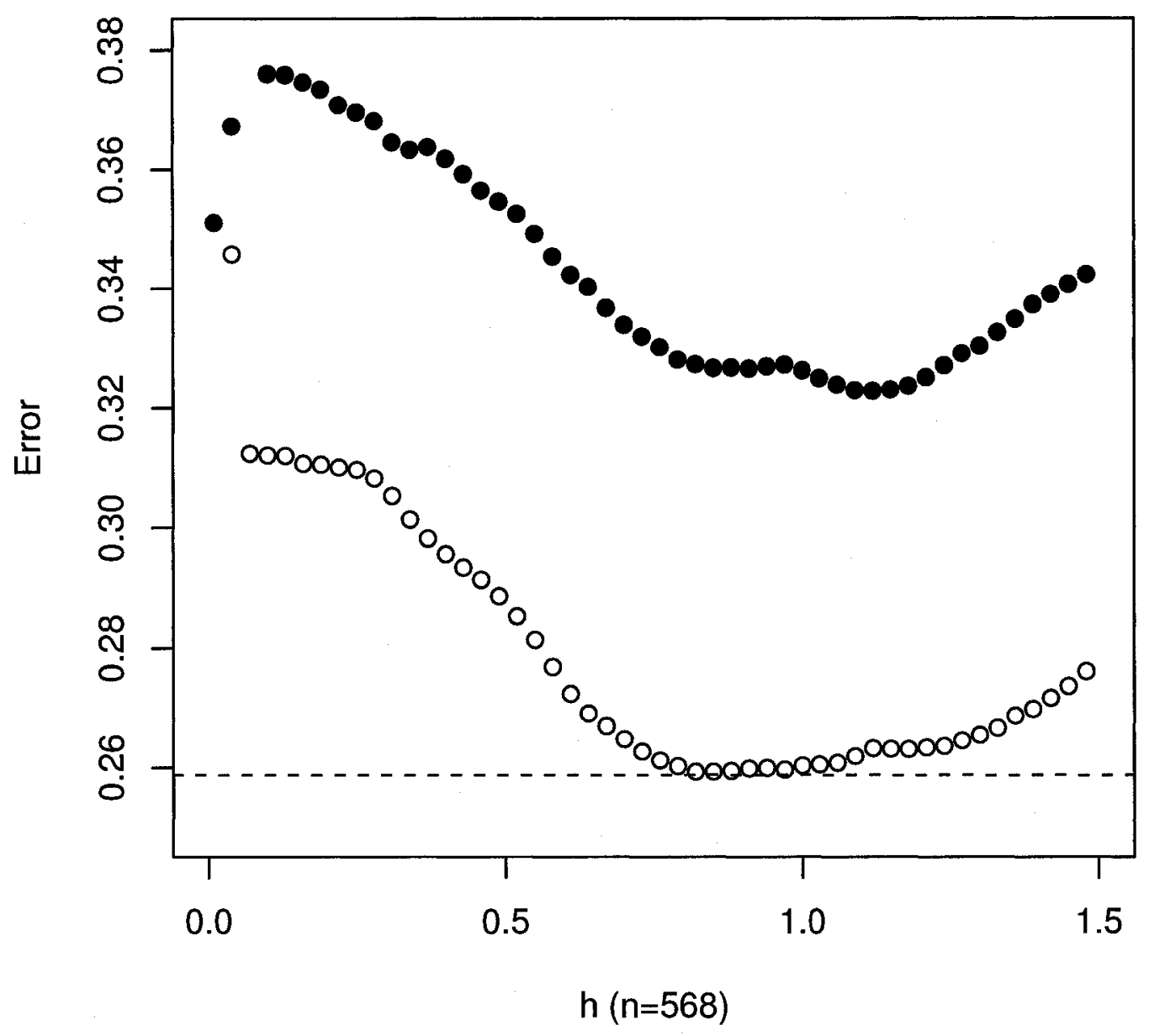


Figure 6.2: Plot of $h$ versus the error, o represents $\psi_{n}^{\mathrm{ker}}, \bullet \operatorname{represents} \widetilde{\psi}_{n}^{\mathrm{ker}}$.

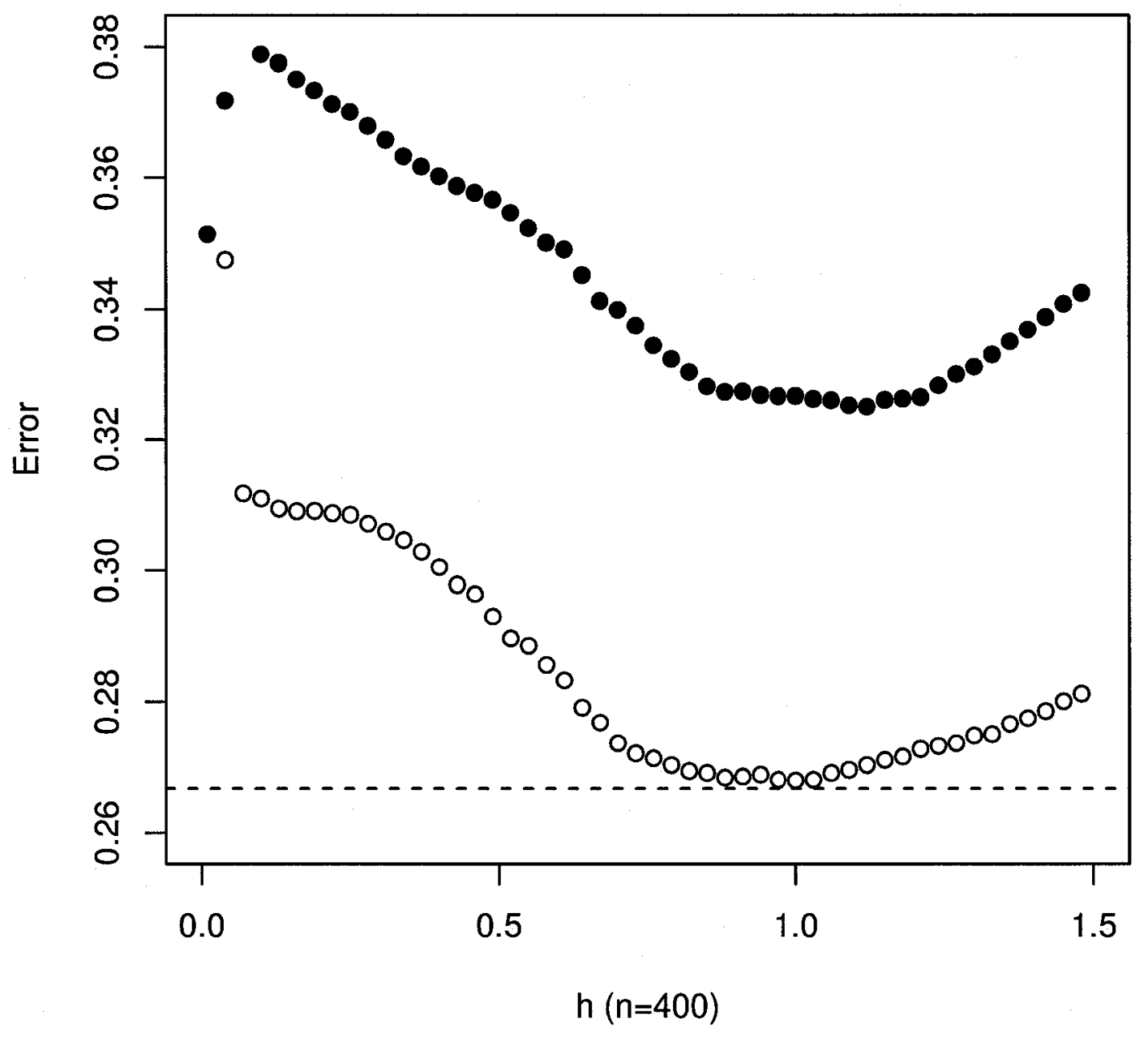


Figure 6.3: Plot of $h$ versus the error, o represents $\psi_{n}^{\mathrm{ker}}, \bullet$ represents $\widetilde{\psi_{n}^{\mathrm{ker}}}$.

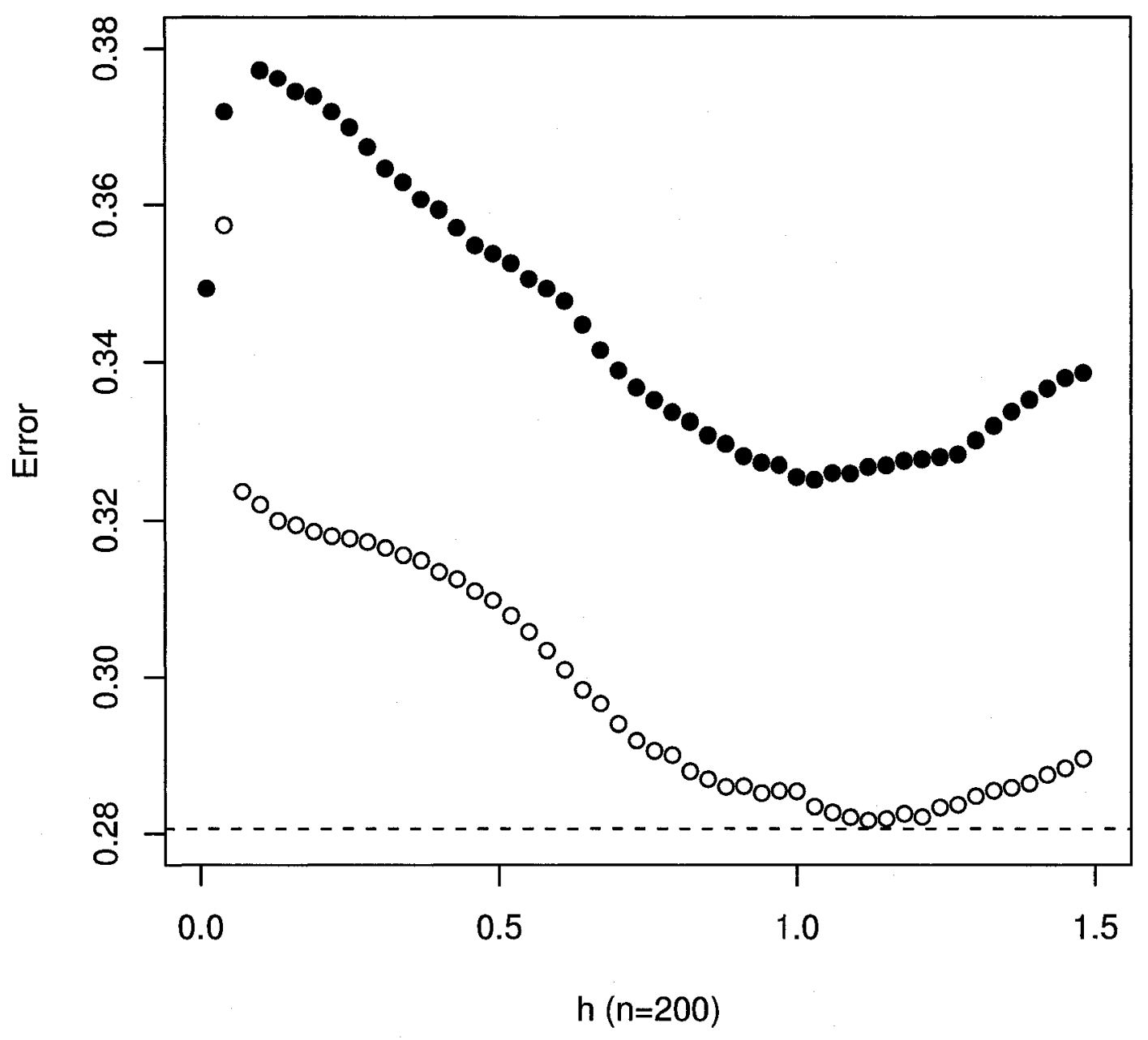

169 


\section{Chapter 7}

\section{Future Research}

This research has a new look on the problem of classification in the presence of missing covariates. This is done by deriving general representations for the best classifier without imposing any assumptions on the underlying missing probability mechanism. In Chapters 2, 3, and 4, we constructed nonparametric classifiers by applying the kernel and histogram rules. These rules were shown to perform well without requiring any MAR-type assumptions. In the parametric cases (Chapters 2 and 3), the MAR assumption was a must. Whether this assumption can be relaxed remains to be an interesting question/area for new developments.

In Chapter 5, we used the least-squares method to pick the empirically best classifier and, to handle the presence of missing covariates, we employed kernel-based imputation as well as Horvitz-Thompson-Type inverse weighting approaches. There are a wide range of future studies based on the methods of this chapter. These include using the kernel-based imputation or the Horvitz-Thompson approaches to handle the presence of missing data when applying other methods of classification. 
More specifically, it will be interesting to see whether the ERM (Empirical Risk Minimization) method of Vapnik-Chervonenkis (see; for example Devroye et al. Chapter 12) can be tailored to fit the current setup with missing covariates. The exponential bound that we found for the kernel-based imputation was based on $\mathcal{N}_{\infty}$ which is the cardinality of the smallest $\epsilon$-cover of $\mathcal{M}$ (the class of the candidate functions $\left.\eta: \mathcal{R}^{p} \rightarrow[0,1]\right)$. Since $\mathcal{N}_{\infty}$ can be a large number, a major challenge would be to find an exponential bound based on the empirical $L_{1}$-norm which is more common in the literature on empirical processes than $\mathcal{N}_{\infty}$. Note that in this case the assumption of independence is jeopardized, which is crucial to symmetrization arguments. Finally in Chapter 5, we had to impose the MAR-assumption for our proposed methods. The question then is whether it is possible to relax this restriction.

The results of this thesis work under the assumption that the dimension of the covariate vector $\mathbf{Z}$ is fixed and does not vary with $n$. An important direction of the future research on classification with missing covariates is the case of high dimensional data where the dimension of $\mathbf{Z}$ is allowed to increase with $n$. This is indeed a major challenge. 


\section{Bibliography}

Afifi, A.A. and Elashoff, R.M. (1966). Missing observation in multivariate statistics: Review of the literature, Journal of the American Statistics Association, 61:595-604.

Anderson, T.W. (1957). Maximum likelihood estimates for the multivariate normal distribution when some observations are missing. Journal of the American Statistical Association, 52:200-203.

Anderson, T.W. (1984). An Introduction to Multivariate Statistical Analysis, John Wiley, New York.

Bartlett, M.S. (1937). Some examples of statistical methods of research in agriculture and applied botany. Journal of royal Statistical Society, B, 4:137-170.

Bartlett, P.L. and Mendelson, S. (2002). Rademacher and Gaussian complexities: risk bounds and structural results. Journal of Machine Learning research, 3:463-482

Bartlett, P.L., Bousquet, O. and Mendelson, S. (2005). Local rademacher complexities, Annals of Statistics, 33:1497-1537.

Bernstein, S. (1946). The Theory of Probabilities. Gastehizdat Publishing House, Moscow. 
Bohannon, Tom R. and Smith, W.B. (1975). Classification based on incomplete data records. ASA Proc. Social Statistics Section, 214-218

Chan, L.S. and Dunn, O.J. (1972). The treatment of missing values in discriminant analysis-1, The sampling experiment, Journal of the American Statistical Association, 67:214-218.

Chan, L.S. and Dunn, O.J. (1974). A note on the asymptotical aspect of the treatment of missing values in discriminant analysis, Journal of the American Statistical Association, 69:672-673

Chen, H. Y. (2004). Nonparametric and semiparametric models for missing covariates in parametric regression. Journal of the American Statistical Association, 99:1176-1189.

Cheng, P.E. (1994). Nonparametric estimation of mean functional with data missing at random. J. Am. Statist. Assoc, 89:81-87.

Cheng, P.E. and Chu, C.K. (1996). Kernel estimation of distribution functions and quantiles with missing data. Sinica, 6:63-78.

Cheng, P.E. and Wei, L.J. (1986). Nonparametric inferences under ignorable missing data process and treatment assignment. International Statistics symposium, Taipei, Vol 1, Institute of Statistical Science, Academia Sinica, Taipei:97-112. 
Chung, H.C. and Han, C.P. (2000). Discriminant analysis when a block of observations is missing. Ann. Inst. Statist. Math. 52:544-556.

Dempster, A.P., Laird, N.M. and Rubin, D.B. (1977). Maximum likelihood from incomplete data via the EM algorithm. J.of the Royal Statistical Society, ser B, 39:1-38.

Devroye, L. and Györfi, L. (1983). Distribution-free exponential bound on the $L_{1}$ error of partitioning estimates of a regression function. In Proceedings of the Fourth Pannonian Symposium on Mathematical Statistics, Konecny, F., Mogyorodi, J., and Wertz, W., editors, pages 67-76. Akademiai Kiado, Budapest, Hungary.

Devroye, L., Györfi, L. (1985). Nonparametric Density Estimation: The $L_{1}$ View, John Wiley, New York.

Devroye, L., Györfi, L., and Lugosi, G. (1996). A Probabilistic Theory of Pattern Recognition. Springer, New York.

Devroye, L. and Krzyzak, A. (1989). An equivalence theorem for $L_{1}$ convergence of the kernel regression estimate. Journal of Statistical Planning and Inference, 23:71-82.

Devroye, L. and Wagner, T. (1980a). Distribution-free consistency results in nonparametric discrimination and regression function estimation. Annals of Statistics, $8: 231-239$. 
Dudley, R. (1978). Central limit theorems for empirical measures. Ann. of Probability, 6:899-929.

Fisher, R. (1936). The case of multiple measurements in taxonomic problems. Annals of Eugenics, 7, part II:179-188.

Fix, E. and Hodges, J. (1951). Discriminatory analysis. Nonparametric discrimination: Consistency properties. Technical Report 4, Project Number 21-49-004, USAF school of Aviation Medicine, Randolph Field, TX.

Fix, E. and Hodges, J. (1952). Discriminatory analysis: small sample performance. Technical Report 21-49-004, USAF school of Aviation Medicine, Randolph Field, TX.

Glick, N. (1973). Sample-based multinomial classification. Biometrics, 29:241-256.

Glick, N. (1976). Sample-based classification procedures related to empiric distributions. IEEE Transactions on Information Theory, 22:454-461.

Györfi, L., Kohler, M., Krzyzak, A. and Walk, H. (2002). A Distribution Free Theory of Nonparametric regression. Springer, New York.

Hartley, H.O. and Hocking, R.R. (1971). The analysis of incomplete data. Biometrics, 27:783-808. 
Hazelton, M.L. (2000). Marginal density estimation from incomplete bivariate data. statistics and Probability Letters, 47:75-84.

Healy, M.J.R. and Westmacott, M. (1956). Missing values in experiments analyzed on automatic computers. Application of Statistics, 5:203-206.

Hirano, K., Imbens, G.W. and Ridder, G. (2003). Efficient estimation of average treatment effects using the estimated propensity score. Econometrica, 71,4:11611189.

Hocking, R.R. and Smith, W.B. (1968). Estimation of parameters in the multivariate normal distribution with missing observation, Journal of the American Statistical Association, 63:159-173.

Hoeffding, W. (1963). Probability inequalities for sums of bounded random variables. Journal of the American Statistical Association, 58:13-30

Horvitz, D.G. and Thompson, D.J. (1952). A generalization of sampling without replacement from a finite population. Journal of the American Statistics Association, 47:663-685.

Kim, J.K. and Fuller, W. (2004). Fractional hot deck imputation. Biometrika, 91:559-578.

Kolmogorov, A.N. and Tikhomirov, V.M. (1959). $\epsilon$-entropy and $\epsilon$-capacity of sets 176 
in function spaces. Uspekhi Math. Nauk, 14:3-86.

Krzyzak, A. (1986). The rates of convergence of kernel regression estimates and classification rules. IEEE Transactions on Information Theory, 32:668-679.

Krzyzak, A. and Pawlak, M. (1984b). Distribution-free consistency of a nonparametric kernel regression estimate and classification. IEEE Transaction on Information theory, 30:78-81.

Little, R.J.A. and Rubin, D.B. (2002). Statistical Analysis with Missing Data. Wiley, New York.

McDiarmid, C. (1989). On the method of bounded differences. In Surveys in Combinatorics 1989, pages 148-188. Cambridge University Press, Cambridge.

McLachlan, G. (2004). Discriminant Analysis and Statistical Pattern Recognition. John Wiley, New York.

Nadaraya, E.A. (1970). Remarks on non-parametric estimates for density functions and regression curves. Theory of Probability and its Applications, 15:134-137.

Nie, N.H., Hull, C.H., Jenkins, J.G., Steinbrenner, K. and Bent, D.H. (1975). SPSS, 2nd edition. McGraw-Hill, New York.

Parzen, E. (1962). On the estimation of a probability density function and mode. 177 
Annals of Mathematical Statistics., 33:1065-1076.

Pollard, D. (1984). Convergence of Stochastic Processes, Springer-Verlag, New York.

Prakasa Rao, B.L.S. (1983). Nonparametric Functional Estimation. Academic Press, London.

Rao, J.N.K. and Wang, Q. (2002). Empirical likelihood-based inference under imputation for missing response data. Annals of Statistics, 30:896-924.

Robins, J.M., Rotnitzky, A., and Zhao, L. (1994). Estimation of regression coefficients when some regressors are not always observed. Journal of the American statistical Association, 89:846-866.

Rosenblatt, M. (1956). Remarks on some non-parametric estimates of density function. Ann. Math. Statist., 27,3:832-837.

Schafer, J.L. (1997). Analysis of Incomplete Multivariate Data. Chapman and Hall, London.

Serfling, R.J. (1980). Approximation Theorem in Mathematical Statistics. Wiley, New York.

Silverman, B.W. (1986). Density Estimation for Statistics and data analysis. Chapman and Hall, London. 
Stone, C.J. (1977). Consistent nonparametric regression. Annals of Statistics, 5:595-645.

Tapia, R.A. and Thompson, J.R. (1978). Nonparametric Probability Density Estimation. The John Hopkins University Press.

van de Geer, S.A. (2000). Empirical Processes in M-Estimation. Cambridge University Press, UK.

van der Vaart, A.W. and Wellner, J.A. (1996). Weak Convergence and Empirical Processes, with Application to Statistics. Springer-Verlag, New York.

Van Ryzin, J. (1966). Bayes risk consistency of classification procedures using density estimation. Sankhya Series A, 28:161-170

Vapnik, V. and Chervonenkis, A. (1971). On the uniform convergence of relative frequencies of events to their probabilities. Theory of probability and its applications, 16:264-280.

Wang, Q., linton, O. and Härdle, W. (2004). Semiparametric regression analysis with missing response at random. Journal of the American Statistical Association, 99:334-345.

Watson, G. (1964). Smooth regression analysis. Sankhya Series A, 26:359-372. 179 
Wilks, S.S. (1932). Moments and distribution of estimates of population parameters from fragmentary samples. Ann. Math. Stat., 3:163-195.

Zhao, L. (1987). Exponential bounds of mean error for the nearest neighbor estimates of regression functions. Journal of Multivariate Analysis, 21:168-178. 